Florida International University FIU Digital Commons

$5-29-2018$

\title{
Elemental Characterization of Printing Inks and Strengthening the Evaluation of Forensic Glass Evidence
}

Ruthmara Corzo

rcorz001@fiu.edu

DOI: $10.25148 /$ etd.FIDC006819

Follow this and additional works at: https://digitalcommons.fiu.edu/etd

Part of the Analytical Chemistry Commons

\section{Recommended Citation}

Corzo, Ruthmara, "Elemental Characterization of Printing Inks and Strengthening the Evaluation of Forensic Glass Evidence" (2018). FIU Electronic Theses and Dissertations. 3814.

https://digitalcommons.fiu.edu/etd/3814 


\section{FLORIDA INTERNATIONAL UNIVERSITY}

Miami, Florida

ELEMENTAL CHARACTERIZATION OF PRINTING INKS AND

STRENGTHENING THE EVALUATION OF FORENSIC GLASS EVIDENCE

A dissertation submitted in partial fulfillment of the

requirements for the degree of

DOCTOR OF PHILOSOPHY

in

CHEMISTRY

by

Ruthmara Corzo

2018 
To: Dean Michael R. Heithaus

College of Arts, Sciences and Education

This dissertation, written by Ruthmara Corzo, and entitled Elemental Characterization of Printing Inks and Strengthening the Evaluation of Forensic Glass Evidence, having been approved in respect to style and intellectual content, is referred to you for judgment.

We have read this dissertation and recommend that it be approved.

David Becker

$\begin{array}{r}\hline \text { Yong Cai } \\ \hline \text { Anthony DeCaprio } \\ \hline \text { Werner Boeglin } \\ \hline \text { Jose Almirall, Major Professor }\end{array}$

Date of Defense: May 29, 2018

The dissertation of Ruthmara Corzo is approved.

Dean Michael R. Heithaus

College of Arts, Sciences and Education

Andrés G. Gil

Vice President for Research and Economic Development and Dean of the University Graduate School

Florida International University, 2018 


\section{DEDICATION}

To my parents, who gave me my first microscope. 


\section{ACKOWLEDGMENTS}

Firstly, I would like to acknowledge Florida International University for awarding me the prestigious Presidential Fellowship. Secondly, I would like to thank my research advisor, Dr. Jose Almirall, for his guidance throughout my research and for always encouraging me to reach higher than I thought possible.

I would also like to thank my committee members Dr. Becker, Dr. Cai, Dr. DeCaprio, and Dr. Boeglin for their time and helpful feedback. A special thank you goes out to Dr. Tatiana Trejos for her eager willingness to assist me with my research. Her passion for teaching constantly motivated me to work harder and strive to be a better scientist.

I am thankful for Pupi and the Almirall research group (the "A team"), in particular the laser lab girls, who made this journey easier to bear. I am grateful to have had constant companions to laugh with and do Zumba with during challenging times.

I would like to acknowledge the US Secret Service for providing ink specimens. I would also like to thank Vidia (who helped analyze glass samples) and all those who participated in the Glass Interpretation Working Group (GIWG).

Last, but certainly not least, I am grateful to my family and friends for their unwavering support. In particular, I would like to acknowledge my parents Susy and Mario, my brother Isaac, my sister Elie, my best friend Kathy, my sister-in-law Mari, and my niece Evie for much needed distractions during stressful times. 


\begin{abstract}
OF THE DISSERTATION
ELEMENTAL CHARACTERIZATION OF PRINTING INKS AND

STRENGTHENING THE EVALUATION OF FORENSIC GLASS EVIDENCE
\end{abstract}

\author{
by
}

\title{
Ruthmara Corzo
}

Florida International University, 2018

Miami, Florida

Professor Jose Almirall, Major Professor

Improvements in printing technology have exacerbated the problem of document counterfeiting, prompting the need for analytical techniques that better characterize inks for forensic analysis. In this study, 319 printing inks (toner, inkjet, offset, and intaglio) were analyzed directly on the paper substrate using Scanning Electron MicroscopyEnergy Dispersive Spectroscopy (SEM-EDS) and Laser Ablation-Inductively Coupled Plasma-Mass Spectrometry (LA-ICP-MS). As anticipated, the high sensitivity of LAICP-MS resulted in excellent discrimination (> 99\%) between ink samples originating from different sources. Moreover, LA-ICP-MS provided $\geq 90 \%$ correct association for ink samples originating from the same source. SEM-EDS resulted in good discrimination for toner and intaglio inks (> 97\%) and excellent correct association (100\%) for all four ink types. However, the technique showed limited utility for the discrimination of inkjet and offset inks.

A searchable ink database, the Forensic Ink Analysis and Comparison System (FIACS), was developed in order to provide a tool that allows the analyst to compare a questioned 
ink sample to a reference population. The FIACS database provided a correct classification rate of $94-100 \%$ for LA-ICP-MS and 67-100\% for SEM-EDS.

An important consideration in forensic chemistry is the interpretation of the evidence. Typically, a match criterion is used to compare the known and questioned sample. However, match criteria suffer from several disadvantages, which can be overcome with an alternative approach: the likelihood ratio (LR). Two LA-ICP-MS glass databases were used to evaluate the performance of the LR: a vehicle windshield database (420 samples) and a casework database (385 samples). Compared to the match criterion, the likelihood ratio led to improved false exclusion rates $(<1.5 \%)$ and similar false inclusion rates $(<$ $1.0 \%$ ). In addition, the LR limited the magnitude of the misleading evidence, providing only weak support for the incorrect proposition.

The likelihood ratio was also tested through an inter-laboratory study including ten LAICP-MS participants. Good correct association rates (94-100\%) were obtained for samesource samples for all three inter-laboratory exercises. Moreover, the LR showed a strong support for an association. Finally, all different-source samples were correctly excluded with the LR, resulting in no false inclusions. 


\section{TABLE OF CONTENTS}

CHAPTER

PAGE

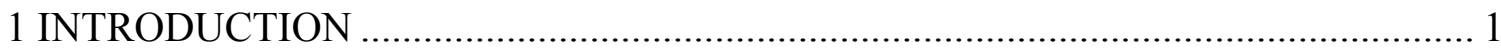

1.1 Research Motivation ......................................................................................... 1

1.2 Significance of Study ........................................................................................ 4

1.3 Composition, Manufacture, and Elemental Analysis of Printing Inks ………......... 5

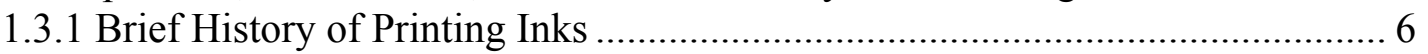

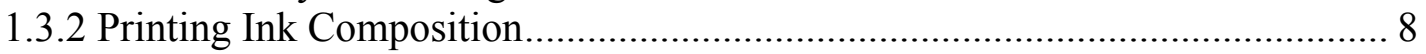

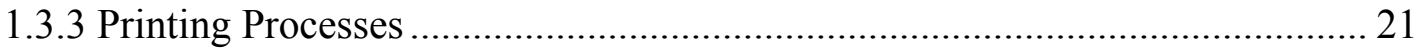

1.3.4 Elemental Analysis of Printing Inks .............................................................. 30

1.4 Composition, Manufacture, and Elemental Analysis of Glass ………………....... 33

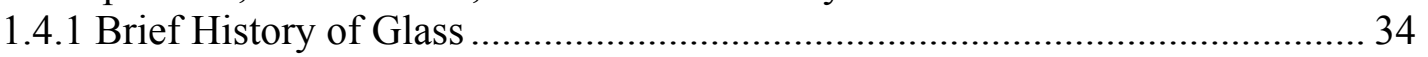

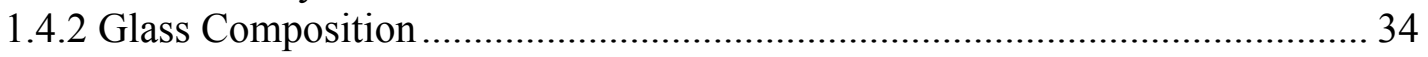

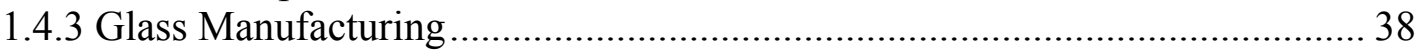

1.4.4 Elemental Analysis of Glass ........................................................................ 41

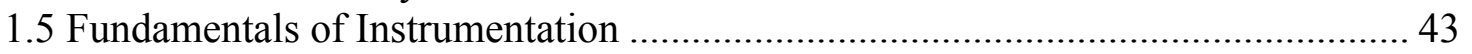

1.5.1 Scanning Electron Microscopy-Energy Dispersive Spectroscopy ................... 43

1.5.2 Laser Ablation-Inductively Coupled Plasma-Mass Spectrometry .................... 47

1.5.3 Laser Induced Breakdown Spectroscopy ………………………….............. 53

1.6 Interpretation of Forensic Evidence.................................................................... 59

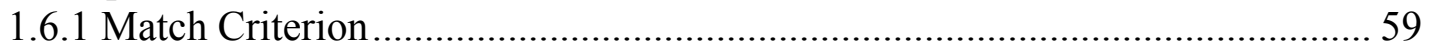

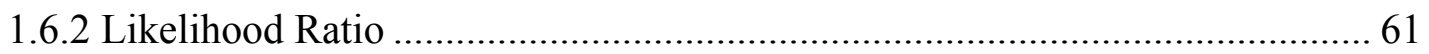

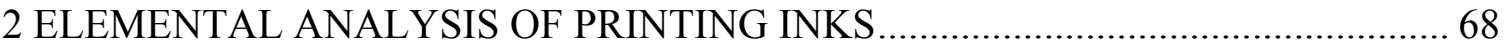

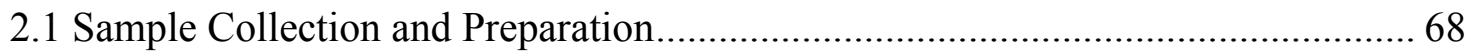

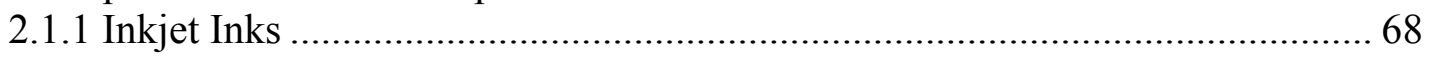

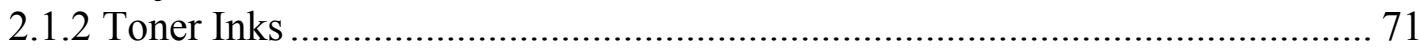

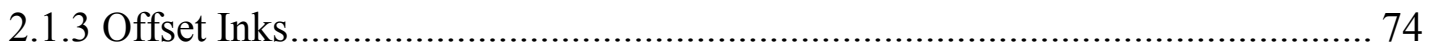

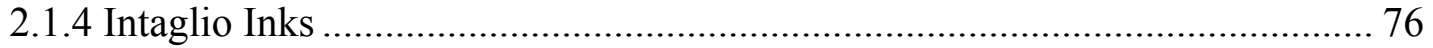

2.2 SEM-EDS Analysis of Printing Inks ................................................................. 79

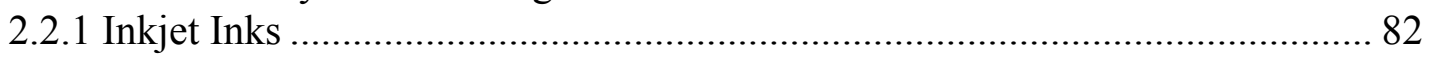

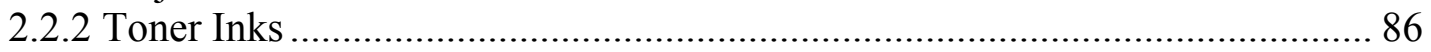

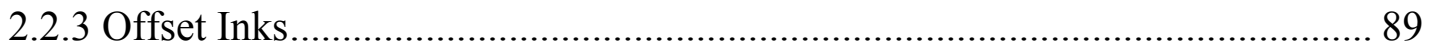

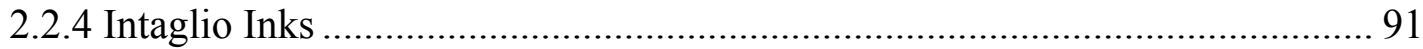

2.3 Comparison of SEM-EDS and LA-ICP-MS Results ............................................. 93

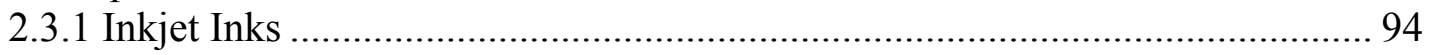

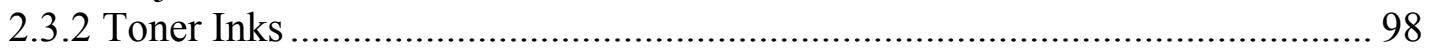

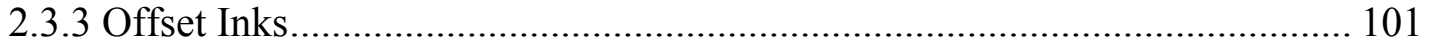

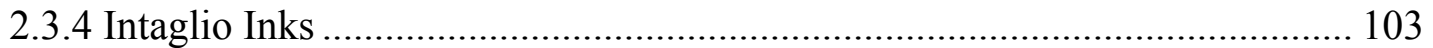

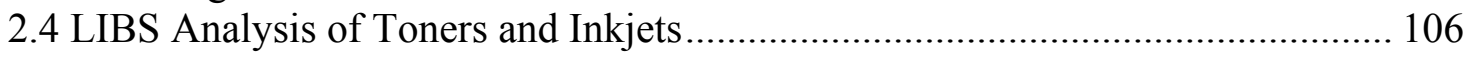

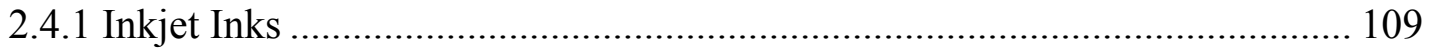

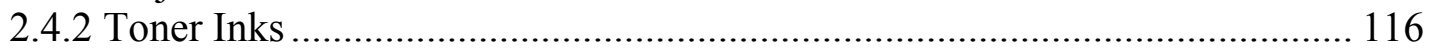

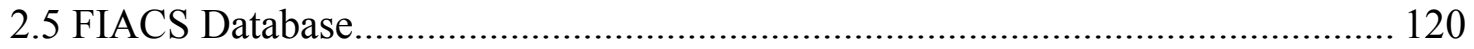




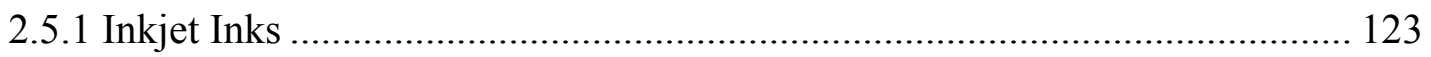

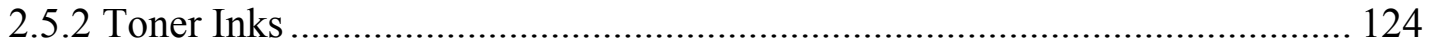

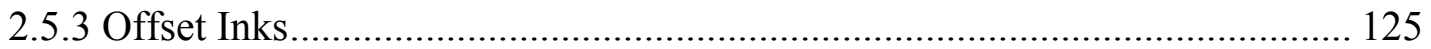

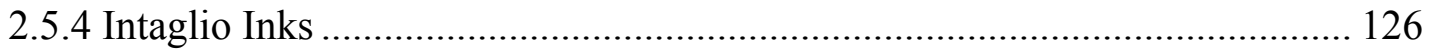

2.6 Conclusions for Elemental Analysis of Printing Inks........................................... 127

3 LA-ICP-MS ANALYSIS OF GLASS AND COLLECTION OF GLASS

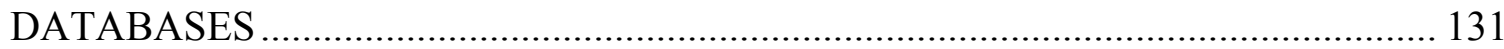

3.1 FIU Vehicle Glass Sample Collection and Preparation....................................... 131

3.2 Elemental Analysis of FIU Vehicle Glass using LA-ICP-MS............................ 135

3.3 Performance of Control Samples ....................................................................... 137

3.4 Figures of Merit and Descriptive Statistics for the FIU Vehicle Glass

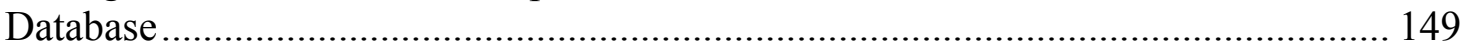

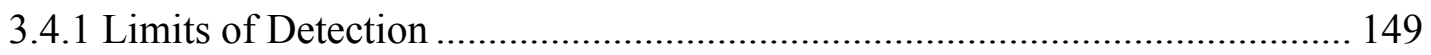

3.4.2 Descriptive Statistics for FIU Vehicle Database ........................................... 153

3.5.1 BKA Casework Database ...................................................................... 156

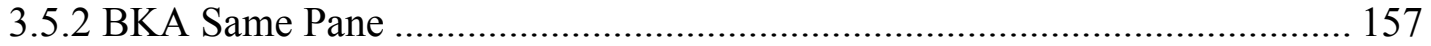

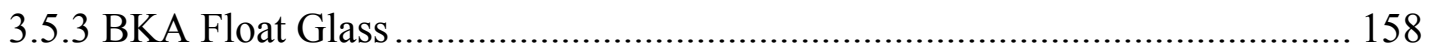

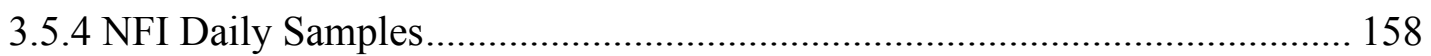

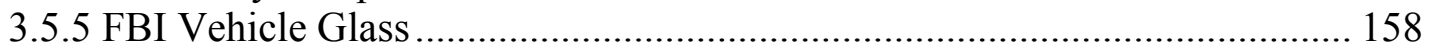

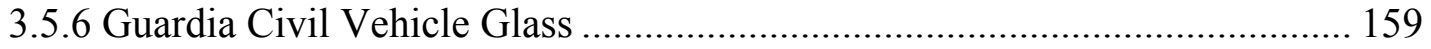

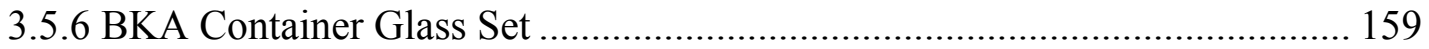

3.6 Conclusions for LA-ICP-MS Analysis of Glass and Collection of Glass

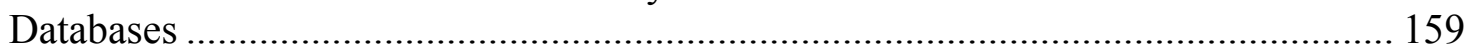

4 ANALYSIS AND INTERPRETATION OF LA-ICP-MS GLASS DATA ................. 161

4.1 Performance of ASTM E2927 Comparison Criterion ………………………...... 161

4.1.1 Description of ASTM E2927 Comparison Criterion........................................ 161

4.1.2 Comparison Criterion Results for FIU Vehicle Database............................... 162

4.1.3 Comparison Criterion Results for BKA Casework Database ........................ 165

4.2 Frequentist versus Bayesian Approach ............................................................ 165

4.2.1 Multivariate Kernel (MVK) Model ......................................................... 167

4.2.2 Calibration of Likelihood Ratio ……………............................................ 167

4.3 Performance of Calibrated Likelihood Ratio for FIU and BKA Database........... 169

4.3.1 Double 10-Fold Cross Validation using the MVK + PAV Approach ............ 169

4.3.2 Double 10-Fold Cross Validation using MVK + PAV Approach with Limited Databases.......................................................................................... 183

4.3.3 Double 10-Fold Cross Validation using the MVK + ELUB Approach.......... 185

4.4 Performance of Likelihood Ratio for Additional Test Datasets ............................. 188

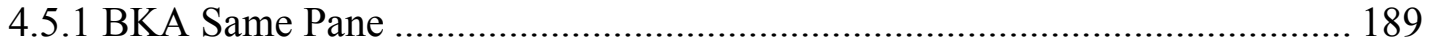

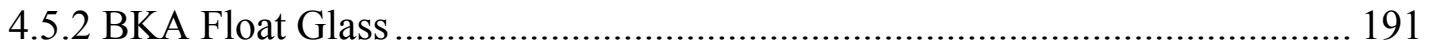

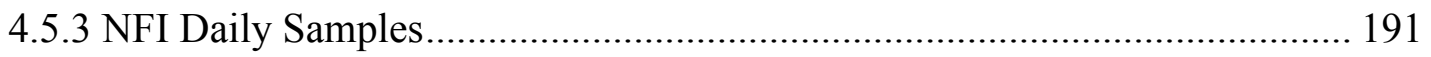

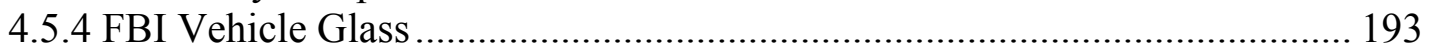

4.5.5 Guardia Civil Vehicle Glass ........................................................................ 193

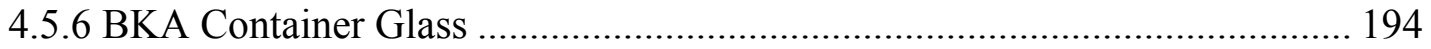


4.5 Conclusions for Analysis and Interpretation of LA-ICP-MS Glass Data

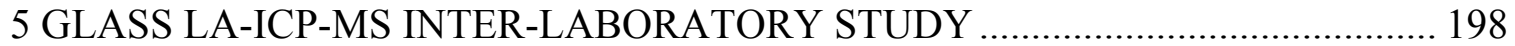

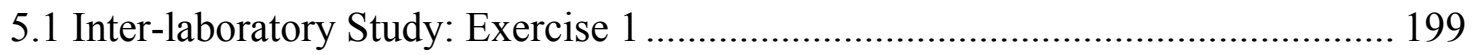

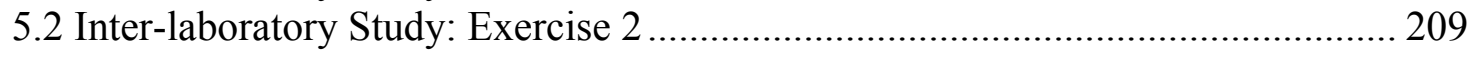

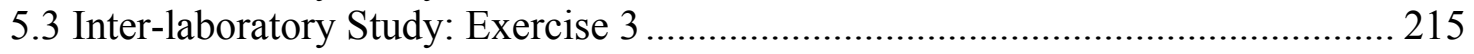

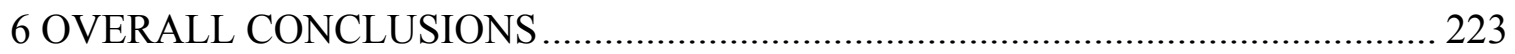

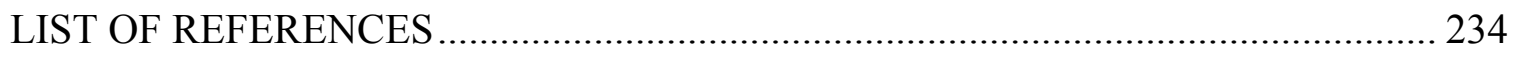

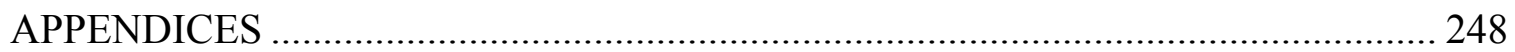

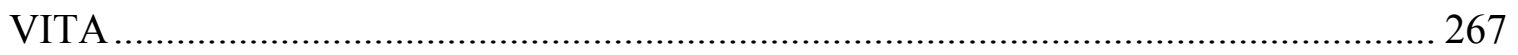




\section{LIST OF TABLES}

TABLE

PAGE

Table 1 - Examples of pigments classified based on their color 9

Table 2 - Examples of dyes from four classifications: acid, basic, solvent, and disperse dyes

Table 3 - Examples of solvents classified by chemical group ................................... 16

Table 4 - Examples of synthetic, petroleum, and natural waxes................................. 17

Table 5 - Characteristics of two-component and mono-component toners ................... 26

Table 6 - Ink patents indicating the role of specific elements in the formulation........... 31

Table 7 - Common formers, fluxes, and stabilizers used in glass formulations ............. 35

Table 8 - Typical composition of container glass and flat glass ................................. 37

Table 9 - Excitation and ionization processes in the inductively coupled plasma .......... 51

Table 10 - Summary of the main characteristics of the four ink types: inkjet, toner,

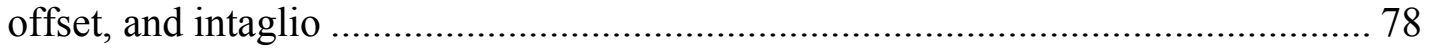

Table 11 - Optimized SEM-EDS parameters for the analysis of printing inks; elements detected $(\mathrm{SNR} \geq 3)$ in the ink and paper are also reported ...................... 80

Table 12 - Discrimination (bolded), correct association (bolded), and false inclusion/exclusion rate for all SEM-EDS inkjet comparisons as well as for each individual color. Values in parentheses indicate either the number of pairwise comparisons or the fraction of discriminated/associated pairs.

Table 13 - Discrimination (bolded), correct association (bolded), and false inclusion/exclusion rate for all SEM-EDS toner comparisons as well as for each individual color. Values in parentheses indicate either the number of pairwise comparisons or the fraction of discriminated/associated pairs

Table 14 - Optimized LA-ICP-MS parameters for the analysis of printing inks; elements detected $(\mathrm{SNR} \geq 3)$ in the ink and paper are also reported.....

Table 15 - Discrimination (bolded), correct association (bolded), and false inclusion/exclusion rate for all LA-ICP-MS inkjet comparisons as well as for each individual color. Values in parentheses indicate either the number of pairwise comparisons or the fraction of discriminated/associated pairs 
Table 16 - Discrimination (bolded), correct association (bolded), and false inclusion/exclusion rate for all LA-ICP-MS toner comparisons as well as for each individual color. Values in parentheses indicate either the number of pairwise comparisons or the fraction of discriminated/associated pairs.

Table 17 - Optimized LIBS parameters and elements detected for each ink type

Table 18 - Discrimination (bolded), correct association (bolded), and false inclusion/exclusion rate for all LIBS inkjet comparisons as well as for each individual color. Values in parentheses indicate either the number of pairwise comparisons or the fraction of discriminated/associated pairs.

Table 19 - Discrimination (bolded), correct association (bolded), and false inclusion/exclusion rate for all LIBS toner comparisons as well as for each individual color. Values in parentheses indicate either the number of pairwise comparisons or the fraction of discriminated/associated pairs.

Table 20 - PLSDA and KNN correct association for inkjet inks using SEM-EDS and LA-ICP-MS data

Table 21 - PLSDA and KNN correct association for toner inks using SEM-EDS and LA-ICP-MS data.

Table 22 - PLSDA and KNN correct association for offset inks using SEM-EDS

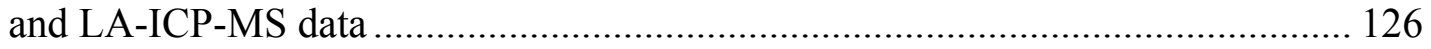

Table 23 - PLSDA and KNN correct association for intaglio inks using SEM-EDS and LA-ICP-MS data.....

Table 24 - Typical LA-ICP-MS analysis sequence for FIU vehicle glass collection .... 137

Table 25 - FGS 1 percent bias for each element over the course of 39 analysis days (values in red have a magnitude greater than $15 \%$, while values in yellow have a magnitude between $10 \%$ and $15 \%$ ).....

Table 26 - NIST 1831 percent bias for each element over the course of 39 analysis days (values in red have a magnitude greater than $15 \%$, while values in yellow have a magnitude between $10 \%$ and $15 \%$ ).

Table 27 - Reference concentration (ppm) used for each calibration standard

Table 28 - Limit of detection (LOD) in parts per million (ppm) and FGS 1 bias for each element.

Table 29 - False inclusion and exclusion for FIU database and duplicates using ASTM comparison criterion 
Table 30 - False exclusion rate, false inclusion rate, Cllr and Cllr $r_{\text {min }}$ (if applicable) for the BKA and FIU databases using different comparison criteria. Fractions within parentheses indicate the number of pairs that were falsely excluded/included over the total number of pairwise comparisons.

Table 31 - Percent of falsely inclusions that originate from the same vehicle make and/or year of manufacture for the FIU database. The fractions within parentheses indicate the number of false inclusions over the total number of different-source pairwise comparisons.

Table 32 - Weight of evidence for false inclusions for each database. The fractions within parentheses indicate the number of pairs that fall within a particular likelihood ratio range (given in the leftmost column) over the total number of different-source pairwise comparisons.

Table 33 - Error rates for the BKA, FIU, and combined database before and after the removal of problematic samples

Table 34 - Comparison of error rates using the PAV and the ELUB calibration method for the limited FIU and the limited BKA database

Table 35 - Distribution of LRs, false inclusion rate, and false exclusion rate for each independent dataset provided by the BKA

Table 36 - Identity of Known and Questioned samples included in round robin 1 ....... 200

Table 37 - Strength of association or discrimination for round robin $1 \mathrm{~K} 2$ outer and Q2

Table 38 - Identity of Known and Questioned samples included in round robin 2 ....... 209

Table 39 - Strength of association or discrimination for round robin $2 \mathrm{~K} 1$ and Q1 ...... 212

Table 40 - Identity of Known and Questioned samples included in round robin 3 ....... 215

Table 41 - Strength of association or discrimination for round robin $3 \mathrm{~K} 1$ and Q1 ...... 217 


\section{LIST OF FIGURES}

FIGURE

PAGE

Figure 1 - Schematic of continuous inkjet printing system...................................... 22

Figure 2 - Schematic of electrophotographic printing system .................................. 24

Figure 3 - Schematic of single-color sheet-fed offset press ................................... 28

Figure 4 - Schematic of intaglio printing unit ........................................................ 30

Figure 5 - Arbogast method (top) and I.S. method (bottom) of manufacturing containers

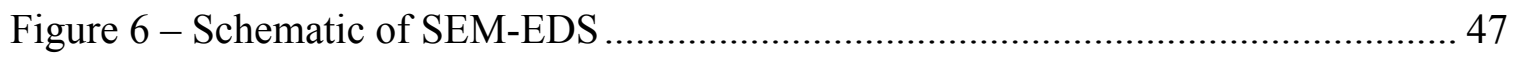

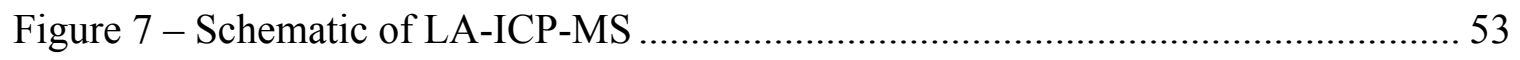

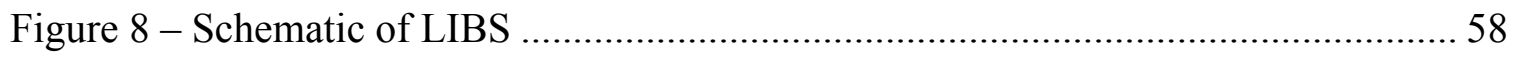

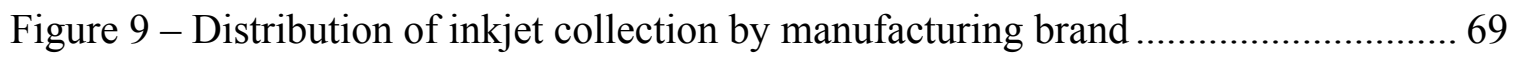

Figure 10 - Distribution of inkjet collection by ink color .......................................... 70

Figure 11 - Example of inkjet specimens: printed (left), smeared with cartridge sponge (middle), and pipetted with micropipette (right).............................................. 71

Figure 12 - Distribution of toner collection by manufacturing brand .......................... 72

Figure 13 - Distribution of toner collection by ink color .................................... 73

Figure 14 - Examples of toner specimens: printed (left), heated and smeared powder (middle), and heated and smeared liquid (right) .............................................. 74

Figure 15 - Distribution of offset collection by sample type ................................... 75

Figure 16 - Examples of offset specimens: printed (left), smeared raw paste (middle), and

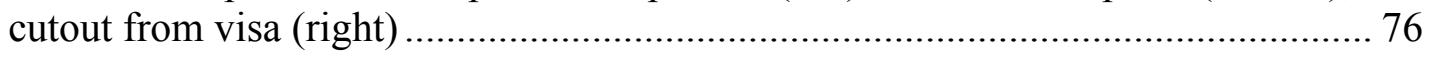

Figure 17 - Distribution of intaglio collection by country of origin ........................... 77

Figure 18 - Barbados banknote with the intaglio areas that were sampled circled in red 78

Figure 19 - Example of two discriminated inkjet samples and Whatman 42 paper (top) and example of one sample compared to its duplicate (bottom) ............................ 83 
Figure 20 - SEM micrograph of inkjet (top left) and examples of discoloration of magenta (top and bottom right), cyan (bottom left), and yellow (bottom middle) inks

Figure 21 - Example of two toners that are distinguishable.

Figure 22 - SEM micrographs of two toners that were indistinguishable by their elemental profile, but distinguishable by their particle morphology ..... 88

Figure 23 - Example of two offset inks that are easily distinguished; the paper blank is also included to demonstrate the high concentration of calcium

Figure 24 - SEM micrographs of two offset samples..................................................... 90

Figure 25 - Examples of three intaglio inks that were easily distinguished..................... 92

Figure 26 - SEM micrographs of two intaglio samples, one with particulates (left) and the other with metallic flakes (right)...................................................................... 92

Figure 27 - Comparison of two inkjet samples that were indistinguishable by LA-ICP-MS (left), but distinguishable by SEM-EDS (right) on the basis of the sulfur and chlorine content 95

Figure 28 - Example of inkjet and paper mass spectra 97

Figure 29 - Comparison of two toner samples that were indistinguishable by LA-ICP-MS (top), but distinguishable by SEM-EDS (bottom) on the basis of the silicon and zinc

Figure 30 - Comparison of two offset samples that were indistinguishable by LA-ICPMS (top), but distinguishable by SEM-EDS (bottom) on the basis of the silicon and titanium

Figure 31 - Pair of intaglio samples that were indistinguishable (top) and three intaglio samples that were distinguishable by unusual elements (bottom) ........................... 104

Figure 32 - Examples of unusual elements detected in banknote paper . 105

Figure 33 - Definitive Screening Design to optimize LIBS parameters for inkjet analysis

Figure 34 - Prediction Profiler in JMP showing parameter values expected to provide the best compromise between SNR and RSD for sodium 108

Figure 35 - Raw (left) versus normalized (right) spectra for a pair of quality controls (top) and a pair of duplicates (bottom) 
Figure 36 - LIBS control charts using element ratios for an inkjet control sample analyzed 8 times over 3 days

Figure 37 - Selected LIBS control charts using element ratios for a toner control sample

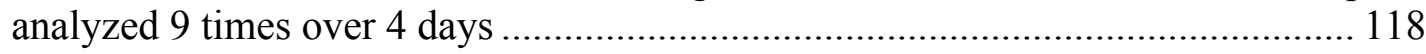

Figure 38 - Example of FIACS PLSDA and KNN output........................................ 120

Figure 39 - Close up of FIACS PLSDA output for duplicate Toner 37..................... 121

Figure 40 - Close up of FIACS KNN output for duplicate Toner 37......................... 122

Figure 41 - Example of windshield sticker disclosing glass manufacturer, (B.) side view of laminated glass, (C.) example of large piece of glass cut from windshield, (D.) small glass fragments broken from outer windshield pane, and (E.) small glass fragments broken from inner windshield pane

Figure 42 - Relative frequency of automobile make for windshield glass in FIU collection set. 133

Figure 43 - Relative percent of unit sales in the United States for each automobile make

Figure 44 - Relative frequency of manufacturing year for windshield glass in FIU collection set

Figure 45 - FGS 1 control charts for each element; black points indicate average daily concentration $(n=5)$.

Figure 46 - NIST 1831 control charts for each element; black points indicate average daily concentration $(n=5)$.

Figure 47 - Trial 1 external calibration curves for selected elements ........................ 152

Figure 48 - Box and whisker plot for FIU vehicle database ...................................... 153

Figure 49 - Correlation between elements for FIU vehicle database ......................... 155

Figure 50 - Box and whisker plot for BKA casework database ................................ 157

Figure 51 - Examples of ECE plots in which the validation set of LRs is: (a.) well calibrated, (b.) better calibrated, and (c.) badly calibrated (Zadora, et al. ${ }^{42}$, figure reproduced with permission from Wiley \& Sons, Ltd)......

Figure 52 - Schematic of double 10-fold calibration procedure using FIU Inner Panes as an example 
Figure 53 - Un-calibrated (left panels) and calibrated (right panels) $\log 10$ LRs for the BKA database (top), the FIU database (middle), and the FIU database with the BKA database as the background population (bottom).

Figure 54 - Un-calibrated (left panels) and calibrated (right panels) ECE plots for the BKA database (top), the FIU database (middle), and the FIU database with the BKA database as the background population (bottom) 176

Figure 55 - Scaled T Distribution (top), QQ plot for the Scaled T Distribution (middle), and Normalized Bayes Error rate plot (bottom) for the BKA database (left panels) and the FIU database (right panels)

Figure 56 - Stacked column plot showing the distribution of LRs for the NFI Daily Samples set. The different colors represent the magnitude of the LR from a strong association (green) to a moderately strong exclusion (red). Note that the LRs are expressed in $\log _{10}$ in the legend.

Figure 57 - Calibrated LR for all round robin $1 \mathrm{~K}$ and Q comparisons using the BKA database as the background population............................................................. 202

Figure 58 - Calibrated LR for all round robin $1 \mathrm{~K}$ and Q comparisons using the FIU database as the background population. 203

Figure 59 - Calibrated LR for all round robin $1 \mathrm{~K}$ and Q comparisons using the combined BKA and FIU database as the background population ..................................... 204

Figure 60 - Round robin 1 NIST 1831 control charts for selected elements ................ 208

Figure 61 - LR results for all round robin $2 \mathrm{~K}$ and Q comparisons using the BKA (top), FIU (middle), and combined (bottom) database as the background population.... 210

Figure 62 - Round robin 2 FGS 1 control charts for selected elements 214

Figure 63 - LR results for all round robin $3 \mathrm{~K}$ and Q comparisons using the BKA (top), FIU (middle), and combined (bottom) database as the background population.... 216

Figure 64 - Round robin 3 FGS 1 control charts for selected elements 219 
ABBREVIATIONS AND ACRONYMS

\begin{tabular}{|c|c|}
\hline ANOVA & Analysis of Variance \\
\hline ASTM & American Society for Testing and Materials \\
\hline BKA & Bundeskriminalamt \\
\hline BSE & Backscattered Electrons \\
\hline CCD & Charge Coupled Device \\
\hline Cllr & Cost Log-Likelihood Ratio \\
\hline CMYK & Cyan Magenta Yellow Black \\
\hline DART-MS & Direct Analysis in Real Time-Mass Spectrometry \\
\hline DOE & Design of Experiment \\
\hline EAWG & Elemental Analysis Working Group \\
\hline $\mathrm{ECE}$ & Empirical Cross Entropy \\
\hline ELUB & Empirical Lower and Upper Bound \\
\hline EU & Expected Utility \\
\hline FBI & Federal Bureau of Investigation \\
\hline FGS & Float Glass Standard \\
\hline FIACS & Forensic Ink Analysis and Comparison System \\
\hline FIU & Florida International University \\
\hline FRSD & Fixed Relative Standard Deviation \\
\hline FTIR & Fourier Transform Infrared Spectroscopy \\
\hline FWHM & Full Width at Half Maximum \\
\hline GIWG & Glass Interpretation Working Group \\
\hline
\end{tabular}




$\begin{array}{ll}\mathrm{H}_{\mathrm{d}} & \text { Defense's Hypothesis }\left(\text { also } \mathrm{H}_{2}\right) \\ \mathrm{H}_{\mathrm{p}} & \left.\text { Prosecutor's Hypothesis (also } \mathrm{H}_{1}\right) \\ \text { ICP-AES } & \text { Inductively Coupled Plasma-Atomic Emission Spectroscopy } \\ \mathrm{K} & \text { Known (control sample) } \\ \text { KDE } & \text { Kernel Density Estimation } \\ \text { KNN } & \text { K-Nearest Neighbor } \\ \text { LA-ICP-MS } & \text { Laser Ablation-Inductively Coupled Plasma-Mass Spectrometry } \\ \text { LIBS } & \text { Laser Induced Breakdown Spectroscopy } \\ \text { LOD } & \text { Limit of Detection } \\ \text { LR } & \text { Likelihood Ratio } \\ \text { LTE } & \text { Local Thermodynamic Equilibrium } \\ \text { MVK } & \text { Multivariate Kernel } \\ \text { MVN } & \text { Multivariate Normal } \\ \text { NA } & \text { Not Applicable } \\ \text { NAA } & \text { Neutron Activation Analysis } \\ \text { NBE } & \text { Normalized Bayes Error } \\ \text { Nd:YAG } & \text { Neodymium: Yttrium Aluminum Garnet } \\ \text { NFI } & \text { Netherlands Forensic Institute } \\ \text { NIST } & \text { National Institute of Standards and Technology } \\ \text { NITECRIME } & \begin{array}{l}\text { Natural Isotopes and Trace Elements in Criminalistics and } \\ \text { Environmental Forensics }\end{array} \\ \text { Original Equipment Manufacturer } \\ \text { Pool Adjacent Violators }\end{array}$




$\begin{array}{ll}\text { PDA } & \text { Photodiode Array } \\ \text { PLSDA } & \text { Partial Least Squares Discriminant Analysis } \\ \text { py-GC-MS } & \text { Pyrolysis Gas Chromatography-Mass Spectrometry } \\ \text { QD } & \text { Questioned (recovered sample) } \\ \text { RSD } & \text { Questioned Document } \\ \text { SD } & \text { Relative Standard Deviation } \\ \text { SE } & \text { Standard Deviation } \\ \text { SEM-EDS } & \text { Secondary Electrons } \\ \text { SNR } & \text { Signal to Noise Ratio } \\ \text { TLC } & \text { Thin Layer Chromatography } \\ \text { XRF } & \text { X-Ray Fluorescence Spectroscopy }\end{array}$




\section{INTRODUCTION}

\subsection{Research Motivation}

The proliferation of inexpensive, high-quality printers has greatly enabled the production of fraudulent documents that closely resemble authentic documents. ${ }^{1}$ A questioned document refers to any document that is suspected to be counterfeit; this may include authentic documents with unsanctioned alterations or apocryphal documents whose source is called into question. Typical specimens encountered in questioned document cases include: checks, envelopes, wills, contracts, passports, IDs, and banknotes. ${ }^{2-3}$ Crimes concerning documents can significantly impact the nation's economy, involving billions of dollars in losses yearly. ${ }^{3}$

The methods traditionally employed in questioned document analysis include physical examinations (e.g., morphological characteristics and striations) and optical examinations using filters and irradiation with UV or IR light sources. ${ }^{2,4-9}$ However, these techniques are limited since different ink samples may exhibit similar physical and optical properties. Therefore, analytical tools that are more selective and sensitive than traditional methods may be necessary to distinguish ink samples originating from different sources.

Current analytical methods utilized for the analysis of printing inks include: Thin Layer Chromatography (TLC), Gas Chromatography-Mass Spectrometry (GC-MS), High Performance Liquid Chromatography (HPLC), Raman Spectroscopy, Fourier Transform Infrared Spectroscopy (FTIR), and Pyrolysis Gas Chromatography-Mass Spectrometry (py-GC-MS), all of which characterize the organic components in the ink, while X-Ray 
Fluorescence Spectroscopy (XRF) and Scanning Electron Microscopy-Energy Dispersive Spectroscopy (SEM-EDS) characterize the inorganic components. ${ }^{10-33}$ Typically, analytical techniques that characterize the organic components in inks are used in forensic laboratories, leaving elemental analysis an underutilized technique for the discrimination of inks. ${ }^{2}$

The diversity of the inorganic components in inks may be a result of contaminants in the raw materials used during the manufacturing process or to the materials that are added intentionally as part of the ink formulation: driers, extenders, coloring agents (pigments and dyes), and other additives. SEM-EDS and XRF have previously been applied for the elemental analysis of printing inks. ${ }^{17,24,30-31}$ SEM-EDS offers non-destructive elemental analysis as well as imaging capabilities; however, the technique is limited because of its poor sensitivity (LOD $\sim 1000 \mathrm{ppm}$ ) and its long acquisition times. ${ }^{29} \mathrm{XRF}$ also provides non-destructive elemental analysis, but with improved sensitivity (LOD $\sim 100 \mathrm{ppm}$ ) compared to SEM-EDS; nevertheless, XRF may be of limited use for ink analysis since the x-ray beam penetrates deeper into the substrate than the electron beam used in SEMEDS, thus the XRF analytical signal may be dominated by the paper background. More recently, Laser Ablation-Inductively Coupled Plasma-Mass Spectrometry (LA-ICP-MS) has been applied to the analysis of printing inks. ${ }^{29,}{ }^{34-35}$ LA-ICP-MS provides greater sensitivity (LOD $<1 \mathrm{ppm}$ ) and is quasi-destructive, requiring the removal of only a small amount (microgram quantities) of sample. A relatively recent analytical tool, Laser Induced Breakdown Spectroscopy (LIBS), provides a comparatively inexpensive alternative to LA-ICP-MS and has the advantages of rapid analysis times and ease of 
operation. Moreover, LIBS has been shown to provide discrimination comparable to LAICP-MS for the analysis of printing inks. ${ }^{29}$

An important consideration in trace analysis is the evaluation of the evidence. Usually, a match criterion (e.g., t-test, range overlap, $n$-sigma) is used to compare the known and questioned sample. ${ }^{36-38}$ The two samples are considered to be indistinguishable if no differences in their elemental profile are found. On the hand, the known and questioned samples are considered to be distinguishable if at least one element is found to differ. The match criterion approach, referred to as the "frequentist approach," has several disadvantages: it suffers from the "fall off the cliff" effect, in which a small change in the evidence can lead to a drastic change in the final decision; it does not account for the rarity of an elemental profile; and it does not provide a weight of evidence. ${ }^{39-42}$

The Bayesian approach is an alternative method for evidence interpretation that does not suffer from the disadvantages stated above. Bayes theorem is defined as:

$$
\frac{P\left(H_{1} \mid E\right)}{P\left(H_{2} \mid E\right)}=\frac{P\left(E \mid H_{1}\right)}{P\left(E \mid H_{2}\right)} \times \frac{P\left(H_{1}\right)}{P\left(H_{2}\right)}
$$

Hypothesis one, $\mathrm{H}_{1}$, supports an association (i.e., no difference between the known and unknown sample), and hypothesis two, $\mathrm{H}_{2}$, supports no association. The first term is known as the posterior odds, the middle term is the likelihood ratio (LR) and the right term is known as the prior odds. To a forensic scientist, only the likelihood ratio is typically of interest. ${ }^{43}$ The LR is the ratio of the probability of the evidence (E) given $\mathrm{H}_{1}$ divided by the probability of the evidence given $\mathrm{H}_{2}$. A LR greater than 1 therefore supports $\mathrm{H}_{1}$, while a LR less than 1 supports $\mathrm{H}_{2}$; if the LR equals 1, neither hypothesis is supported. Moreover, a larger LR shows stronger support for an association and a smaller 
LR shows stronger support for an exclusion (non-association). Unlike the frequentist approach, the LR provides a quantitative and more objective approach to evidence interpretation. The LR has been applied to many types of forensic evidence including, but not limited to: glass, paint, gunshot residue, fingerprints, illicit drugs, DNA, and speaker recognition. ${ }^{44-68}$

\subsection{Significance of Study}

A primary goal of my dissertation was to evaluate and compare the performance of SEMEDS and the more sensitive technique LA-ICP-MS for the analysis of four types of printing inks: inkjet, toner, offset, and intaglio. Over 300 ink specimens were collected and analyzed using each technique. The complementarity of the two techniques as well as the discrimination and correct association capabilities were evaluated. A subset of inkjets and toners were also analyzed using LIBS, a relatively new technique that has shown promise for the analysis of inks.

A second goal of the ink project involved the development of a searchable ink database that allows a user to compare a questioned ink sample to a reference collection. The purpose of the Forensic Ink Analysis and Comparison System (FIACS) is to narrow down the list of possibilities for the origin of the questioned sample. FIACS uses Partial Least Squares Discriminant Analysis (PLSDA) and K-Nearest Neighbor (KNN) to compare the questioned sample to the reference collection and identify candidate ink sources. Currently, the database is populated with known samples of authentic origin. However, the reference collection can be expanded to include fraudulent documents of 
known origin. As such, the database can serve as a novel forensic tool to potentially identify the origin of an unknown counterfeit document.

Evidence interpretation is an important aspect of forensics. In the United States, a match criterion is used to compare a known and questioned glass sample. However, in the event of an association, a match criterion does not consider the strength of the association. The final part of this research is the evaluation of an alternative approach to evidence interpretation, the likelihood ratio. Although the LR approach can be used for many types of evidence, glass was selected as the model matrix since LA-ICP-MS analysis of glass has been extensively studied. ${ }^{36-37,69-73}$ A total of 420 windshield glass samples were collected and analyzed using LA-ICP-MS. Additionally, a 385-sample casework glass database was provided by the Bundeskriminalamt (BKA) in Germany. The quantitative data for each database was used to calculate a likelihood ratio through a cross validation study. This research presents the first study to directly compare the match criterion currently in use in the United States to the relatively new likelihood ratio. Additionally, an inter-laboratory study was conducted in order to test the performance of the likelihood ratio in mock case scenarios. The inter-laboratory study was part of an ongoing effort to standardize the interpretation of forensic evidence as well as the reporting language used in case reports.

\subsection{Composition, Manufacture, and Elemental Analysis of Printing Inks}

Questioned Document (QD) analysis refers to the examination of documents that are suspected of being counterfeit. Examples include forged checks, forged or altered wills, and counterfeited banknotes. The role of a QD analyst can include the comparison of a 
questioned (recovered) handwriting sample to a known (control) handwriting sample, comparison of a recovered paper sample to a control paper sample, and/or comparison of a recovered ink sample to a control ink sample. Suspect documents are typically examined using visual and optical methods since these methods are non-destructive. However, the analysis of the organic components in the ink may be used to characterize the ink or to date the ink. ${ }^{3}$ Since the present study focuses solely on the analysis of printing inks, only this type of ink will be described in detail.

\subsubsection{Brief History of Printing Inks}

The earliest evidence of printing comes from Chinese records around $251 \mathrm{CE}$. The Chinese used hand-carved wooden blocks and water-based writing ink that was adapted for printing purposes. This printing style remained unique for the next 1300 years and later became known as the letterpress process after the invention of other printing processes. $^{74}$

Printing reached Europe during the Middle Ages. At this time, the high demand for religious texts and the works of poets and writers led to the search for a more efficient method of making copies. The introduction of paper, which appeared in Italy during the $12^{\text {th }}$ century, made the shift from writing to printing possible. Prior to the availability of paper, texts were written on parchment made from sheepskin or goatskin. The manufacture of parchment in sufficiently large quantities to allow printing was extremely expensive. By the $14^{\text {th }}$ century, paper was quickly replacing parchment for most purposes, with the exception of legal documents. At this time, books were copied using whole-page wooden blocks, which were inked with water-based writing inks that were 
used for the original manuscripts. However, this procedure was still cumbersome, which led to a search for a form of movable type. The Chinese had attempted this as early as $1041 \mathrm{CE}$, but discontinued their efforts because of the large number of characters required..$^{74}$

In the $15^{\text {th }}$ century, Johann Gutenberg, a goldsmith, developed a process of casting metallic type that produced high-quality characters. The ink played an important role in Gutenberg's success. Water-based ink was unsuitable because it would not properly adhere to the metallic type, resulting in poorly defined printed characters. At around the same time, a technique for producing paint using linseed oil and litharge reappeared in Europe; this technique had been used in Roman times, but had been lost to Europe until the $15^{\text {th }}$ century. Gutenberg produced his own oil-based formulation. Although the complete formulation is unknown, some components are known: linseed oil, walnut oil, turpentine, rosin, pitch, Venice turps, lamp-black, and vermillion. Gutenberg's new ink produced sharp characters that had intense black color, which was an improvement to the washy brown color of the water-based inks. By the early $17^{\text {th }}$ century, water-based inks were obsolete and all inks were being made with drying oils and resins. ${ }^{74}$

Originally, the printer had both the role of ink making and printing. However, the increasing demand for printed works led to an independent ink maker. The ink maker paved the way to a much greater use of color in printing. His task included the formulation of colored inks, which were made using pigments obtained from minerals that had to be finely ground. This task was complex since each color required specific proportions of varnishes. ${ }^{74}$ 
Letterpress printing remained the dominant method of printing until the early $1800 \mathrm{~s}$. In the 1790s Senefelder introduced direct lithographic printing, which was later replaced by offset lithography by the end of the 1800s. With each printing technique, the printing ink needed to be modified and more carefully formulated. Synthetic pigments with a much finer texture and more vibrant colors were introduced by the end of the 1800 s. Over the next sixty years, the range of synthetic pigments continued to expand. Two other printing techniques (aniline and photogravure) resulted in the development of quick-drying inks based on volatile solvents. ${ }^{74}$

Throughout the 1930s, research into ink chemistry was initiated in universities and industrial laboratories. The collaboration between universities and industries as well as the development of the petrochemical industry by the end of the 1940s resulted in a rapid expansion in ink technology. At this time, many new pigments, polymers, solvents, and additives were produced, bringing about the rapid end of traditional ink formulations that had changed little over several centuries. From 1950 onwards, the ink industry continued to expand with the introduction of the packaging industry and new technology used to print magazines. Ironically, research in the 1990s focused on water-based ink (the original type of ink used in early printing) because of environmental concerns associated with the ink formulations. ${ }^{74}$

\subsubsection{Printing Ink Composition}

Printing ink is a complex matrix composed of pigments, dyes, oils, resins, solvents, plasticizers, waxes, driers, and other additives. A detailed description of each component is provided in the following sections. 


\subsubsection{Pigments}

Pigments are insoluble multi-molecular crystalline structures that impart color to the ink. When a pigment is applied to a substrate (through a vehicle), they remain on the surface of the substrate or fill in the voids in paper or other irregularly shaped substrates. Some pigments are produced in nature, but the majority of them are synthetic and produced from a variety of materials. Pigments can be classified as either organic or inorganic.

Table 1 lists several examples of different pigments according to their color. Additionally, pearlescent, metallic, and fluorescent pigments are available. ${ }^{74}$

Table 1 - Examples of pigments classified based on their color

\begin{tabular}{cl}
\hline Color & \multicolumn{1}{c}{ Examples } \\
\hline Yellow & $\begin{array}{l}\text { Mono arylide yellows, diarylide yellows, iron oxide yellows, } \\
\text { tartazine yellow lake, chrome yellows, cadmium yellow }\end{array}$ \\
Orange & $\begin{array}{l}\text { DNA orange, pyrazolone orange, diarylide orange, fast orange F2G, } \\
\text { benzimidalozone orange HL, perinone orange }\end{array}$ \\
Red & $\begin{array}{l}\text { Naphthol red, toluidine red, permanent red 'R', carmine F. B., rubine } \\
\text { 2B, lithol reds, lake red 'C', BON maroon, copper ferrocyanide } \\
\text { pink, anthraquinone scarlet }\end{array}$ \\
Green & PMTA deep green, PMTA vivid green, phthalocyanine green, \\
Blue & $\begin{array}{l}\text { PMTA Victoria blue, phthalocyanine blue, milori blue, ultramarine } \\
\text { blue, alkali blue G, indanthrene blue }\end{array}$ \\
Violet & $\begin{array}{l}\text { PMTA rhodamine, quinacridone violet, dioxazine violet, crystal } \\
\text { violet CFA, benzimidazolone bordeux HF 3R, thioindigo red }\end{array}$ \\
Brown & $\begin{array}{l}\text { Brown iron oxide, diazo brown 5R, chromium antimony titanium } \\
\text { buff rutile, benzimidazolone brown HFR }\end{array}$ \\
Black & $\begin{array}{l}\text { Vegetable black (lamp black), carbon black (furnace black and } \\
\text { channel black), black iron oxide }\end{array}$ \\
& $\begin{array}{l}\text { Zinc white, lithopone, titanium dioxide (anatase and rutile), zinc } \\
\text { sulfide, calcium carbonate, china clay, blanc fixe, alumina hydrate, } \\
\text { talc, silica }\end{array}$ \\
\hline
\end{tabular}


The pigment selected for an ink formulation depends on its physical and chemical properties as well as the end use. For example, phthalocyanine blue NC beta form (Color Index Pigment Blue 15:3) is the most commonly used colored pigment, apart from the black and white pigments. This greenish blue pigment is the cyan ink standard for paste and oil formulations and accounts for over $50 \%$ of all Blue 15 production because of its desirable properties: it is resistant to acids, alkalis, solvents, plasticizers, greases, paraffin wax, and soap; and it is solvent-stable, heat-resistive, and very lightfast.

Carbon blacks are the most important black pigments. Their color ranges from grey to jet-black, dependent of the method of manufacture. Carbon blacks are chemically inert and resistive to heat, light, acids, alkalis, solvents, and soap.

White pigments such as zinc white and calcium carbonate can serve the dual purpose of imparting a white color and acting as an extender; extenders are added to ink formulations in order to dilute the color and/or to increase bulk. The most important white pigment is titanium dioxide, which accounts for over $80 \%$ of all whites (including extenders). Like carbon black, titanium dioxide $\left(\mathrm{TiO}_{2}\right)$ is inert and highly resistant. $\mathrm{TiO}_{2}$ has two major crystal polymorphs: rutile and anatase. Rutile is harder, more opaque, and more durable. Anatase is softer, less opaque, less durable, and has a bluer tone.

More information about the properties, uses, and chemical composition of the pigments listed in Table 1 (as well as several additional pigments) can be found in Leach and Pierce. $^{74}$ 


\subsubsection{Dyes}

Dyes, like pigments, impart color to the ink. Dyes are mostly used for liquid inks, coatings, and lacquers, though disperse dyes can be used in both liquid and paste inks used for heat transfer printing. Paste inks can also include acid dyes for double tone and invisible inks as well as security inks in checks. Basic dyes in free-base form are oilsoluble and can be used in toners for black inks.

The Color Index classifies dyes depending on on their specific usage. However, a specific dye may be included in multiple categories. For example, food dyes can include acid dyes, solvent dyes, natural dyes, and pigments. There are 18 usage classifications defined by the Color Index: acid dyes, azoic dyes, basic dyes, developers, direct dyes, disperse dyes, fluorescent brighteners, food and drug dyes, ingrain dyes, leather dyes, mordant dyes, natural dyes, oxidation bases, pigments, reactive dyes, solvent dyes, sulfur dyes, and vat dyes. Table 2 lists examples of dyes from four of the classifications: acid dyes, basic dyes, solvent dyes, and disperse dyes. Leach and Pierce provide more detail for many of the dyes listed in Table $2 .^{74}$

Table 2 - Examples of dyes from four classifications: acid, basic, solvent, and disperse dyes

\begin{tabular}{cl}
\hline Classification & \multicolumn{1}{c}{ Examples } \\
\hline Acid Dyes & $\begin{array}{l}\text { Acid blue 9, acid orange 7, acid red 87, acid yellow 3, acid } \\
\text { yellow 73, acid green 26, acid brown, 355, acid black 47 }\end{array}$ \\
Basic yellow 2, basic yellow 37, basic red 1, basic violet 10, \\
basic blue 26, basic violet 1
\end{tabular}


As the name suggests, acid dyes have the presence of an acidic group. They are anionic dyes, soluble in water, and mostly insoluble in organic solvents. However, some acid dyes are soluble in alcohols, ketones, and esters. Chemically, acid dyes are azo, anthraquinone, triphenylmethane, azine, xanthene, ketonimine, nitro, and nitroso compounds.

Basic dyes are cationic dyes that typically have brilliant shades and high tinctorial strength. However, they exhibit poor light-fastness (i.e., they are prone to discoloration when exposed to light), which limits their usefulness. These dyes are soluble in water and alcohol, but mostly insoluble most other organic solvents.

Solvent dyes are metal-complex dyes and are soluble in organic solvents. Solvent dyes include acid dyes of the azo chromium complex, acid dyes of the xanthene classes, and the base salt form of some basic dyes. Sulfonic groups are typically absent in solvent dyes, with the exception of some alcohol-soluble dyes. Solvent dyes include a wide range of extremely bright colors and have fair light-fastness.

Originally, the term "disperse dyes" referred to water-insoluble azo, diphenylamine, and anthraquinone dyes because these were sold as dispersions. However, modern techniques can produce these as dry re-dispersible powders. Most disperse dyes are primary, secondary, or tertiary amines of three main types: amino azo benzene, aminoanthraquinone, and nitrodiaryl amines. Disperse dyes are mainly used in heat transfer inks for printing on textiles such as synthetic fibers. ${ }^{74}$ 


\subsubsection{Oils}

Oils are one of the oldest raw materials used in ink formulations, yet they still serve an important function in printing ink formulations. Oils are often treated, purified, and/or modified with polymers in order to meet the characteristics required for modern applications. Oils used in printing ink formulations can be classified into several categories: drying vegetable oils, segregated oils, marine oils, semi-drying oils, nondrying oils, news-ink oils, and non-drying vegetable oils. ${ }^{74}$

Drying vegetable oils are glycerides or triglycerides of fatty acids. They are typically characterized by their power to absorb oxygen from the surrounding environment and their ability to form elastic films, which is dependent upon the degree of polymerization. Polymerization occurs when two or more molecules of the same compound combine to form a single molecule. The amount of oxygen absorbed depends on the degree of unsaturation (i.e., the number of double bonds, $-\mathrm{C}=\mathrm{C}-$ ), which indicates the drying power of the oil. Saturated fatty acids play little role in drying while unsaturated fatty acids play a vital role in the drying, color, resistance, and durability of the ink vehicles that are manufactured from particular oils. Examples of drying vegetable oils include: linseed oil, tung oil, oiticica oil, and dehydrated castor oil.

Segregated oils are prepared by treating drying oils with a liquid solvent such as liquid propane, furfural, or a mixture of liquid propane and furfuraldehyde. The solvent segregates the oil into two layers based on the degree of unsaturation. After separation, the solvent is removed through distillation. The segregated oil with the higher degree of unsaturation (i.e., more double bonds) has faster air drying times than the original. 
Marine oils are obtained from the fatty portions of fish and the blubber of whales. These oils have limited use because of their odor, color, drying speeds, cost, and conservation. Semi-drying oils are used as components in synthetic resins. Examples of semi-drying oils include: tobacco seed oil, soybean oil, safflower oil, and sunflower oil. Of these four, soybean oil is the most important.

Non-drying oils includes mineral oil, which is used as a vehicle in printing inks or as a solvent or diluent for the manufacture of ink vehicles. Mineral oils are the higher boiling fractions evaporated from petroleum. These oils have varying amounts of aromatic, naphthenic, and paraffinic hydrocarbons, with low sulfur content $(\leq 4 \%)$. A higher percentage of aromatic and paraffinic hydrocarbons yields poorer pigment wetting properties and poorer miscibility with resins.

News-ink oils must be inexpensive and must show good wetting and dispersion characteristics. Dark brown mineral oils fulfill this requirement. Mineral oils used in newspaper inks generally have a high aromatic content $(\sim 22-44 \%)$.

Finally, non-drying vegetable oils are used as plasticizers, lubricants, and components of synthetic resins. The most important non-drying vegetable oil is castor oil, obtained from Ricinus communis by cold pressing and solvent extraction.

\subsubsection{Resins}

Resins are non-crystalline solid materials or liquids with a relatively high molecular weight. Resins contribute to the hardness, gloss, adhesion, and flexibility of a printing ink. There are two main categories of resins: natural and synthetic. ${ }^{74}$ 
Natural resins are mostly obtained from living vegetable matter or, less often, from fossilized vegetable matter. The chemical composition of natural resins is complex and not fully known. Examples of natural resins include: rosin, shellac, manila copal, asphalts, starch and dextrin, and gum arabic.

Synthetic resins are prepared by polymerization between relatively small molecules via condensation or addition reactions. As such, the chemical composition of synthetic resins is known with high accuracy. Examples of synthetic resins include: pure phenolic resins, rosin-modified phenolic resins, alkyd resins, hydrocarbon resins, polystyrene resins and copolymers, terpene resins, silicone resins, alkylated urea formaldehyde resins, alkylated melamine formaldehyde resins, polyamide resins, polyimide resins, poly(amide-imide) resins, chlorinated rubber, cyclized rubber, vinyl resins, ketone resins, acrylic resins, epoxide resins, polyisocyanates and polyurethanes, nitrocellulose, ethyl cellulose, ethyl hydroxyethyl cellulose, cellulose acetate propionate, cellulose acetate butyrate, and sodium carboxymethyl cellulose.

Leach and Pierce provide a comprehensive description of the natural and synthetic resins listed above. ${ }^{74}$

\subsubsection{Solvents}

The term "solvent" can apply to a large number of solid, liquid, and gaseous substances. A solution is defined as a homogeneous mixture of two or more substances. The solvent is the major constituent of a solution and the solute is the minor constituent of the solution. ${ }^{75}$ The selection of a solvent (or a mixture of solvents) for use in ink formulations depends on the solute(s) that is to be dissolved and the rate of evaporation. Solvents with 
high hydroxyl content are strongly polar and have a high dielectric constant. On the other hand, hydrocarbon solvents and other solvents are nonpolar and have a low dielectric constant. Solvents can be classified according to their chemical properties: hydrocarbons, alcohols, glycols, ketones, and esters. Examples of each group are provided in Table 3. Further information about the properties and uses of the examples listed in Table 3 can be found in Leach and Pierce. ${ }^{74}$

Table 3 - Examples of solvents classified by chemical group

\section{Classification $\quad$ Examples}

Low-boiling petroleum distillates (e.g., white spirit and paraffin

Hydrocarbons oil), high-boiling petroleum distillates, aromatic hydrocarbons (e.g., toluene), high-boiling aromatic solvents

Alcohols Ethanol, propanol, isopropanol, butanol, alicyclic alcohols (e.g., cyclohexanol, methyl cyclohexanol)

Monoethylene glycol, monopropylene glycol, hexylene glycol,

Glycols diethylene glycol, dipropylene glycol, triethylene glycol, glycerine, ethoxy propanols Acetone, methyl ethyl ketone, methyl isobutyl ketone,

Ketones cyclohexanone, methyl cyclohexanone, isophorone, diacetone alcohol

Esters Ethyl acetate, isopropyl acetate, N-butyl acetate, N-propyl acetate

\subsubsection{Plasticizers}

The purpose of a plasticizer is to make the ink more flexible once it has dried. They should mostly be non-volatile in their final ink format. Plasticizers make the dry ink film more elastic by acting as solvents for the film-forming polymer molecules. Typically, plasticizers are viscous liquids, though solid plasticizers are becoming more popular. Some plasticizers can be incorporated into ink formulations to provide specific desired properties in the final ink film such as: high gloss, deep freeze resistance, blocking 
prevention, increased adhesion for difficult substrates, and less discoloration at high temperature. The main classes of plasticizers include: abietates, adipates, benzoates, butyrates, citrates, epoxidized compounds, phthalates, polyesters, polyol esters, ricinoleates, sebacates, stearates, and sulfonamides. ${ }^{74}$

\subsubsection{Waxes}

Waxes are the main components in carbon copying inks and other "hot melt" inks in which printing is done at high temperatures and drying occurs by solidification. Waxes can be introduced into an ink formulation in two ways: adding a wax with a controlled fine particle size to the batch of ingredients or dispersing the wax into a varnish and/or solvent. The non-rub qualities of a wax are dependent on the particle size, hardness, and melting temperature of that particular wax. Adding wax to an ink formulation decreases gloss and increases drying time. Thus there is a compromise between the non-rub qualities of the wax and negative characteristics such as decreased gloss. Table 4 lists examples of synthetic, petroleum, and natural waxes. ${ }^{74}$

Table 4 - Examples of synthetic, petroleum, and natural waxes

\begin{tabular}{cl}
\hline Classification & \multicolumn{1}{c}{ Examples } \\
\hline Synthetic & Polyethylene waxes, polytetrafluoroethylene, fatty acid amides \\
Petroleum & $\begin{array}{l}\text { Slack wax, scale wax, fully refined paraffin wax, petrolatum or } \\
\text { petroleum jelly, microcrystalline waxes, ceresin wax, montan wax, } \\
\text { montan esters }\end{array}$ \\
Natural & $\begin{array}{l}\text { Beeswax, carnauba wax, shellac wax, japan wax, candelilla wax, } \\
\text { lanolin }\end{array}$ \\
\hline
\end{tabular}




\subsubsection{Driers}

Driers are catalysts that promote the oxidation of drying oils (e.g., linseed oil, dehydrated castor oil). With these catalysts, oxidation occurs quickly and the ink films dry hard within a few hours. The most popular driers in use are the inorganic salts and metallic soaps of organic acids. There are two forms of metallic driers: oil-soluble soaps, which encompass the liquid driers and dispersions of inorganic salts in oil, which encompass the paste driers.

Liquid driers are prepared by converting a suitable organic acid into its heavy-metal salt and soap. These compounds are soluble in oils and/or petroleum solvents and form liquids or pastes that mix readily with the oils in the ink. The metals used for liquid driers include: cobalt, manganese, cerium, zirconium, lithium, calcium, zinc, and iron. These metals are typically available as salts of fatty acids such as: octoic fatty acids, rosin fatty acids, naphthenic fatty acids, tall oil fatty acids, and linseed fatty acids. Cobalt is the most powerful and popular metal. However, manganese may be more suitable for whites and tints since cobalt tends to discolor whites and tints. Cerium, zirconium, and lithium have medium efficiency and have replaced lead in many vehicles. These three metals are usually mixed with cobalt or manganese. Iron is useful in tung oil varnishes. Finally, calcium and zinc are ineffective as catalysts and are typically only used in white inks. The metal content in liquid driers is usually in the range of $3 \%$ and $18 \%$, though lead and zirconium can be as high as $36 \%$. Normally, a concentration of about $0.5 \%$ to $4 \%$ of driers is sufficient to achieve drying in printing inks.

Paste driers are prepared by grinding organic salts of lead and manganese in linseed oil varnishes. Lead acetate $\left[\mathrm{Pb}\left(\mathrm{C}_{2} \mathrm{H}_{3} \mathrm{O}_{2}\right)_{2}\right]$ and manganese borate $\left(\mathrm{B}_{2} \mathrm{Mn}_{3} \mathrm{O}\right)$ are commonly 
used. The concentrations vary between formulations but usually about $40 \%$ lead acetate and $8 \%$ manganese borate is used. Paste driers are normally used in multicolor process inks.

\subsubsection{Miscellaneous Additives}

The miscellaneous additives include a variety of components: chelating agents, antioxidants, surfactants, deodorants and reodorants, pure chemicals, defoaming agents, and laking agents.

Chelating agents are chemicals that react with multivalent metal ions, creating extremely stable soluble or insoluble chelates. By binding metal ions, chelating agents can improve the shelf life, color, clarity, and stability of the ink. Chelating agents also improve wetting in water-based inks by softening the water and restricting the activity of iron, calcium, and magnesium salts. Chelates can control the release of metal ions over a prolonged period of time or during a chemical reaction. Moreover, they can suppress a reaction until an elevated temperature is reached. Important chelating agents include: ethylenediaminetetra-acetic acid (EDTA) and its sodium salts, nitriloriacetic acid salts, sodium salts of diethylenetriamine-acetic acid, heptonates, dimethyl glyoxime and its sodium salts, and alkanolamines.

Anti-oxidants react with free radicals until all the anti-oxidant molecules are exhausted. In an ink formulation, anti-oxidants delay the initiation of oxidative polymerization drying. Common anti-oxidants include: naphthols, substituted phenols, oximes, and aromatic amines. 
Surfactants are substances that are adsorbed at surfaces or interfaces, resulting in the reduction of surface or interfacial tension. Soaps and detergents behave as surfactants in aqueous solutions. Surfactants are classified on the basis of their ionic or active component. Anionic surfactants carry a negative charge and attract positively charged molecules, leaving a negative charge at the surface. Cationic surfactants carry a positive charge and attract negatively charged molecules, leaving a positive charge at the surface. Non-ionic surfactants have no residual electric charge and are either lyophilic (solventliking) or lyophobic. Finally, amphoteric surfactants have a balanced positive and negative and can behave as anionic or cationic surfactants depending on the surrounding conditions.

Deodorants and reodorants are used to mask unpleasant odors (particularly from highly volatile solvents). Examples include: amyl and methyl salicylate, vanillin, and blended derivatives of essential oils. Some manufacturers incorporate reodorants in the packaging ink of a product in order to increase sales. Some examples of reodorants used include: lavender, leather, peppermint, carnation, antiseptic, cedarwood, citron, and vanillin.

Pure chemicals include alkalis, organic acids and acid anhydrides, and polyols. Alkalis are mostly used in the formulation of water-based inks. Organic and acid anhydrides are mainly used as the raw materials for the preparation off resins. Finally, polyols are used for the manufacture of synthetic vehicles.

Foaming occurs in ink when a monomolecular film of a surfactant is present on the surface on the liquid. A defoaming agent may be used to inhibit foaming in the ink. Defoaming agents can work in two ways: by acting as a good solvent for the surfactant or 
by providing a small amount of an immiscible material to the system, which reduces surface tension causing bubbles to burst.

Laking agents are required to stabilize basic dyes in water-based or ethanol-based inks. The main laking agent is tannic acid. However, the production of tannic acid from natural sources has become expensive. For this reason, synthetic tannic acid substitutes are more commonly being used. ${ }^{74}$

\subsubsection{Printing Processes}

\subsubsection{Inkjet Printing}

In inkjet printing, droplets or particles are directed in rapid succession towards the substrate (e.g., paper). There are several methods of generating the droplets, but only two methods will be discussed: continuous jet and impulse or drop on demand.

The continuous jet is the most common inkjet system. In this method, the ink is forced under pressure out of one or more nozzles. The liquid jet breaks into a stream of small droplets, the size and frequency of which is determined by the surface tension of the ink, the pressure applied, and the size of the nozzle. Applying a high-frequency alternating voltage to a piezo crystal will produce a stream of droplets with a uniform size and spacing. The stream is projected toward the paper substrate at a speed of $5-20 \mathrm{~m} / \mathrm{s}$, which produces a continuous stream of droplets. To print characters and/or images, the droplets must be individually controlled and deflected. First, the droplets are charged by induction using charge electrodes. The charge that each droplet carries depends on the voltage applied to the charge electrodes. The droplets can then be deflected by the oppositely charged high voltage deflection plates. The extent of deflection is dependent on the 
magnitude of the charge that the droplet carries. For the areas of the paper that are to remain blank, the droplets are left uncharged and are therefore not deflected. These uncharged droplets make their way to the gutter, which collects the unused ink. The unused ink is filtered and returned to the ink reservoir.

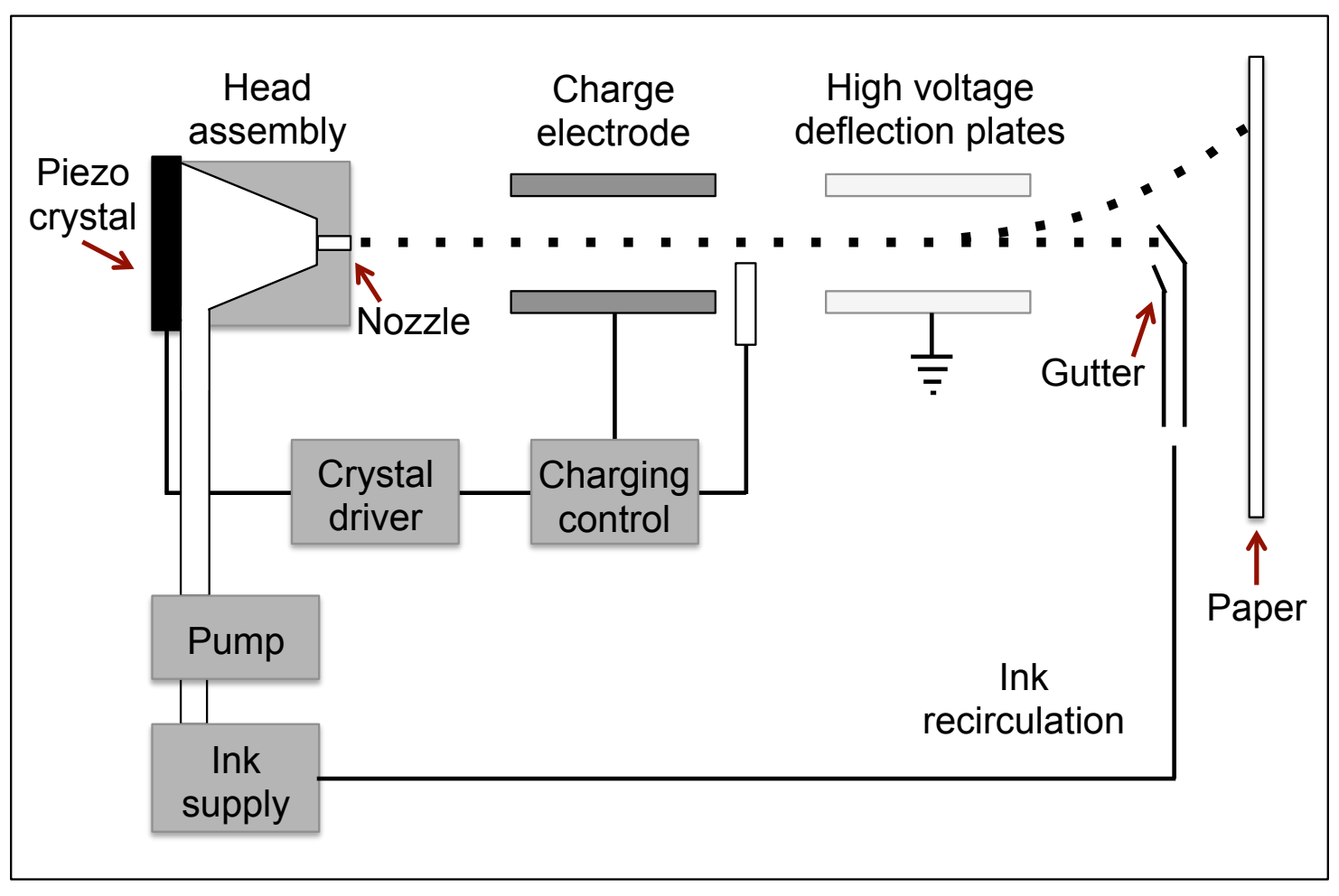

Figure 1 - Schematic of continuous inkjet printing system

Figure 1 shows a schematic of a typical continuous jet inkjet system. Some continuous jet systems have multiple nozzles. Other systems (the Hertz inkjet system) use extremely small nozzles that produce small droplets. The Hertz inkjet system yields higher print quality, but prints very slowly.

The impulse or drop on demand inkjet system does not have a continuous stream of ink droplets. Instead, pressure is applied to the ink reservoir only when droplets are needed to 
form part of a character or image. Therefore, no ink recirculation is needed, making the drop on demand inkjet printer simpler than the continuous inkjet printer. A commonly used system includes an array of 12 nozzles, each with a piezo-electric crystal, so that no deflection is necessary for printing.

Inkjet inks are usually low viscosity inks and contain a solvent blend, colorant, binder, and additive. Five to eight solvents are typically used. An important consideration for inkjet inks is the drying time. For continuous inkjet systems, solvent is lost during the ink recirculation process. Thus these systems must replenish the solvent. Inkjet inks typically use dyes as the colorant, though pigments with a particle size less than $3 \mu \mathrm{m}$ can be used. The dyes must be soluble in the solvent, have high thermal stability, and be lightfast. The binder consists of one or more polymers and is used to adjust the viscosity of the ink and promote good droplet formation. Finally, additives may be added for several purposes: to modify flow properties and surface energy, to plasticize the binder, and to make the ink electrically conductive. The additives account for less than $1 \%$ of the ink formulation. Nonetheless, they are important components of the ink.

\subsubsection{Toner Printing}

Toner printing systems use optical or electrical methods to create a latent image that attracts the toner ink. The ink is then transferred to the substrate. The main types of toner printing systems include: electrophotography, ion deposition, electrostatic, magnetographic, and electrographic.

Electrophotography is often used in photocopiers and laser printers. Figure 2 shows a schematic with the main components of an electrographic printing system. 


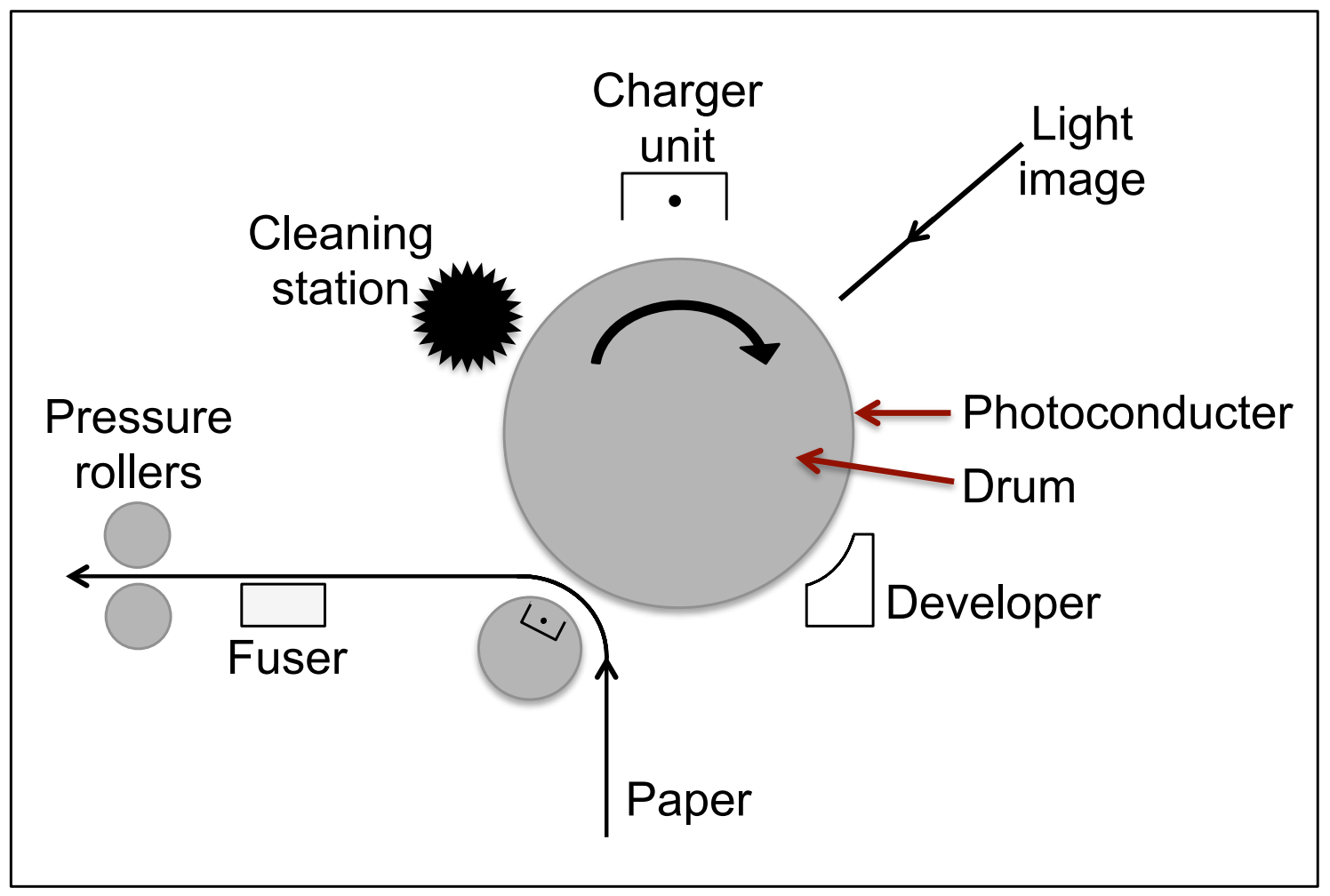

Figure 2 - Schematic of electrophotographic printing system

In electrophotographic printing, the latent image is produced by generating a uniform electric charge on a photoconductive rotating drum. The charged latent image can be created by a laser, a light-emitting diode (LED) array, a liquid crystal array (LCD) with a light source, a magneto-optic array with a light source, or a cathode ray tube with fiber optics. The laser (usually HeNe gas, HeCd gas laser, or diode) is the most common device used to create the latent image. The toner ink is attracted to the charged latent image on the rotating drum. The ink is then transferred to the paper, which moves past the rotating drum at the same speed as the drum. A corona charges the back of the paper so that the toner moves from the rotating drum to the paper. However, this process is not completely efficient and some toner remains on the drum, which needs to subsequently 
be removed. After the toner is transferred to the paper, it must be fused either by heat or heat and pressure.

The ion deposition method is similar to the electrophotographic process. The main difference is that the latent image is formed directly rather than with a light source. The rotating drum is a dielectric material and an ion source array directly deposits charge onto the drum. Fusing of the ink onto the paper takes place only by pressure.

In the electrostatic method, the paper acts as the dielectric medium, which is charged to form the latent image. Liquid toner is applied by a spray or roller as the paper moves past the toner reservoir.

The magnetographic method uses a drum that has a magnetic coating. The image is produced by magnetizing areas on the drum using an array of electromagnets. The magnetized areas attract magnetic toner particles.

In electrographic printing, a latent electroconductive image, called the master image, is formed either by physical methods or by photochemical methods. Multiple identical copies of this image are produced, after which the rest of the printing process is similar to electrophotographic printing. Physical methods of producing the master image include: toner fusion xeroprinting, overcoated relief printing plate, charge retention masters, and "AMEN" and "HEP." Photochemical methods of producing the master image include: photopolymerization/photohardening, photo-induced differential trioelectric potentials, doped photoconductors, silver masters, photoconductors with switching layers, and electrochemography. 
Table 5 - Characteristics of two-component and mono-component toners

\begin{tabular}{lcccl}
\hline Toner Type & $\begin{array}{c}\text { Particle } \\
\text { Size }(\mu \mathrm{m})\end{array}$ & $\begin{array}{c}\text { Resistivity } \\
(\boldsymbol{\Omega} \mathbf{~ c m})\end{array}$ & $\begin{array}{c}\text { Charge } \\
(\mu \mathrm{C} / \mathrm{g})\end{array}$ & \multicolumn{1}{c}{ Composition } \\
\hline $\begin{array}{l}\text { Two- } \\
\text { component }\end{array}$ & $5-32$ & $>10^{13}$ & $10-30$ & $\begin{array}{l}\text { Pigment (usually carbon black), } \\
\text { charge control agent, } 90 \% \\
\text { polymer binder and resin, surface } \\
\text { flow and cleaning additives }\end{array}$ \\
$\begin{array}{l}\text { Conductive } \\
\text { mono- } \\
\text { component }\end{array}$ & $5-45$ & $10^{3}-10^{12}$ & - & $\begin{array}{l}50-70 \% \text { magnetic oxide, polymer } \\
\text { binder, carbon black, cleaning } \\
\text { additive }\end{array}$ \\
$\begin{array}{l}\text { Resistive } \\
\text { mono- } \\
\text { component }\end{array}$ & $5-32$ & $>10^{13}$ & $2-20 \begin{array}{l}30-60 \% \text { magnetic oxide, charge } \\
\text { control agent, polymer binder, } \\
\text { surface flow and cleaning } \\
\text { additives }\end{array}$ \\
\hline
\end{tabular}

Toner ink can either be a dry powder or a dispersion in a liquid. Powdered toners are either two-component toners or mono-component toners. Table 5 lists the characteristics of two-component and mono-component toners.

Two-component toners consist of fine toner pigment particles and a carrier (coarse beads). The toner and carrier are oppositely charged and thus the beads are coated with toner. Two-component toners are typically used in high-speed laser printers and copiers. Mono-component toners fall into two categories: conductive and resistive. Conductive mono-component toners are both magnetic and conductive and have an intermediate particle size. The larger particle size can result in limited printing resolution. Conductive mono-component toners are normally used in ion deposition printing systems. Resistive mono-component toners are similar to the conductive mono-component toners, though they have slightly lower magnetic oxide concentrations. These toners are used in Hewlett Packard Laserjet printers. 
Liquid toners are a colloidal dispersion of fine particles in a highly insulating isoparaffin. They typically have a charge of about $2000 \mu \mathrm{C} / \mathrm{g}$ and a particle size in the range of $0.1-2$ $\mu \mathrm{m}$. The concentration of the particles are in the range of $0.5-1 \%$. The ink formulation includes: pigments, polymers for stabilization and fusing, charge control agents, and a dispersant.

\subsubsection{Offset Lithographic Printing}

Offset lithography is a planographic printing process, in which the image and non-image areas are on the same plane of the image carrier (a flat printing plate). The image carrier is chemically treated so that the ink adheres to specific areas. The non-image areas are water-accepting and thus repel the ink. This printing technique is called "offset lithography" since the inked image on the printing plate is not directly transferred to the paper substrate. Instead, the image is first "offset" onto a rubber blanket and then transferred to the paper. Offset lithographic presses may be sheet fed or web fed; in the former, the paper is in sheet form and in the latter, the paper is fed from a roll. There are many different designs for sheet-fed presses.

Figure 3 shows a schematic of a basic single-color sheet-fed press. The ink rollers apply the ink to the water-repelling areas of the plate cylinder and the damping rollers apply water to the ink-repelling areas of the plate cylinder. The image is transferred to the rubber-coated blanket cylinder. Finally, the image is transferred to the paper, which is fed between the blanket cylinder and the impression cylinder. Sheet-fed presses can include one to six colors. A two-color press consists of two plate cylinders, two offset cylinders, 
and one impression cylinder. A four-color press can either have four one-color presses or two two-color presses.

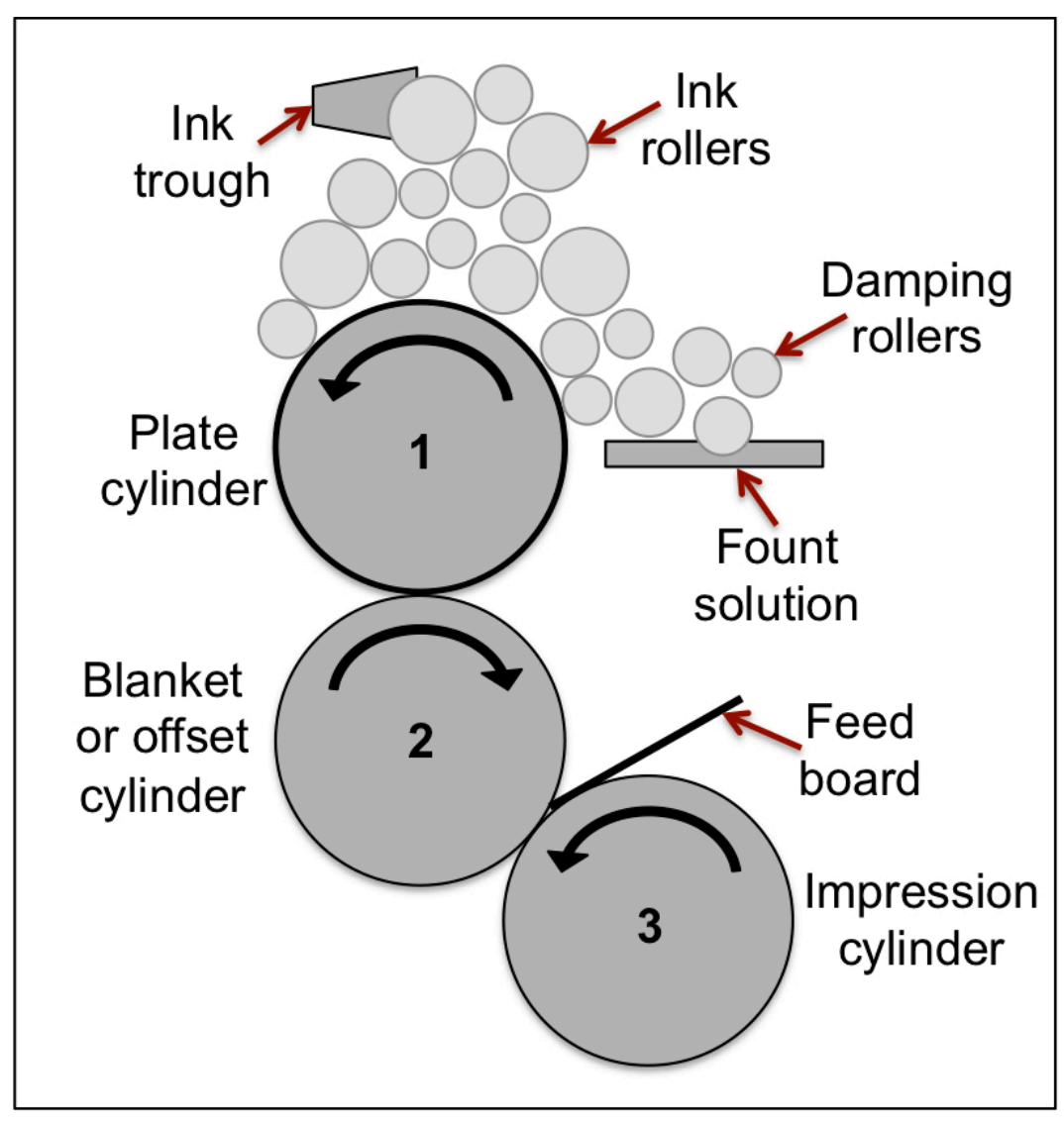

Figure 3 - Schematic of single-color sheet-fed offset press

A wide variety of plates are available for offset printing. The plates that are most commonly used are pre-sensitized plates. A light sensitive material is applied to the base material of the plate (which can be paper, plastic, or aluminum). The two light sensitive materials that are typically used are diazo and photopolymer. Other plates include: wipeon plates, deep-etch plates, multi-metal plates, electrostatic imaged plates, chemical 
diffusion offset plates, photodirect plates, direct image plates, driographic plates, and laser exposed plates. Leach and Pierce provide a brief description of each plate. ${ }^{74}$

Most sheet-fed lithographic inks dry by oxidation, which is aided by absorption on porous materials. On the other hand, web-fed presses use heat-set inks, which dry by evaporation. Some presses use specialized ultra-violet (UV) curing inks. After printing, the UV curing inks are exposed to UV radiation, which rapidly dries the ink. Infra-red (IR) radiation can be used to accelerate drying time; this drying method, unlike UV curing, does not require specialized inks. ${ }^{74}$

\subsubsection{Intaglio Printing}

Intaglio printing, which is commonly used for banknotes, is the process of printing from an engraved plate or cylinder (usually made of steel, copper, or brass). Figure 4 shows a schematic of the intaglio printing process.

The metallic plate or cylinder is completely flooded with thick ink. Any excess ink is removed by a counter-rotating roller, which is then cleaned in a solvent bath. Printing occurs by pressing an impression roller, made of rubber or compressed cotton, against the printing plate or cylinder. The substrate (e.g., paper) passes between the impression roller and the printing plate/cylinder. Strong hydraulic rams are required to achieve the high pressure necessary for printing. The intaglio printing process produces fine tapering lines and a very thick ink film. 


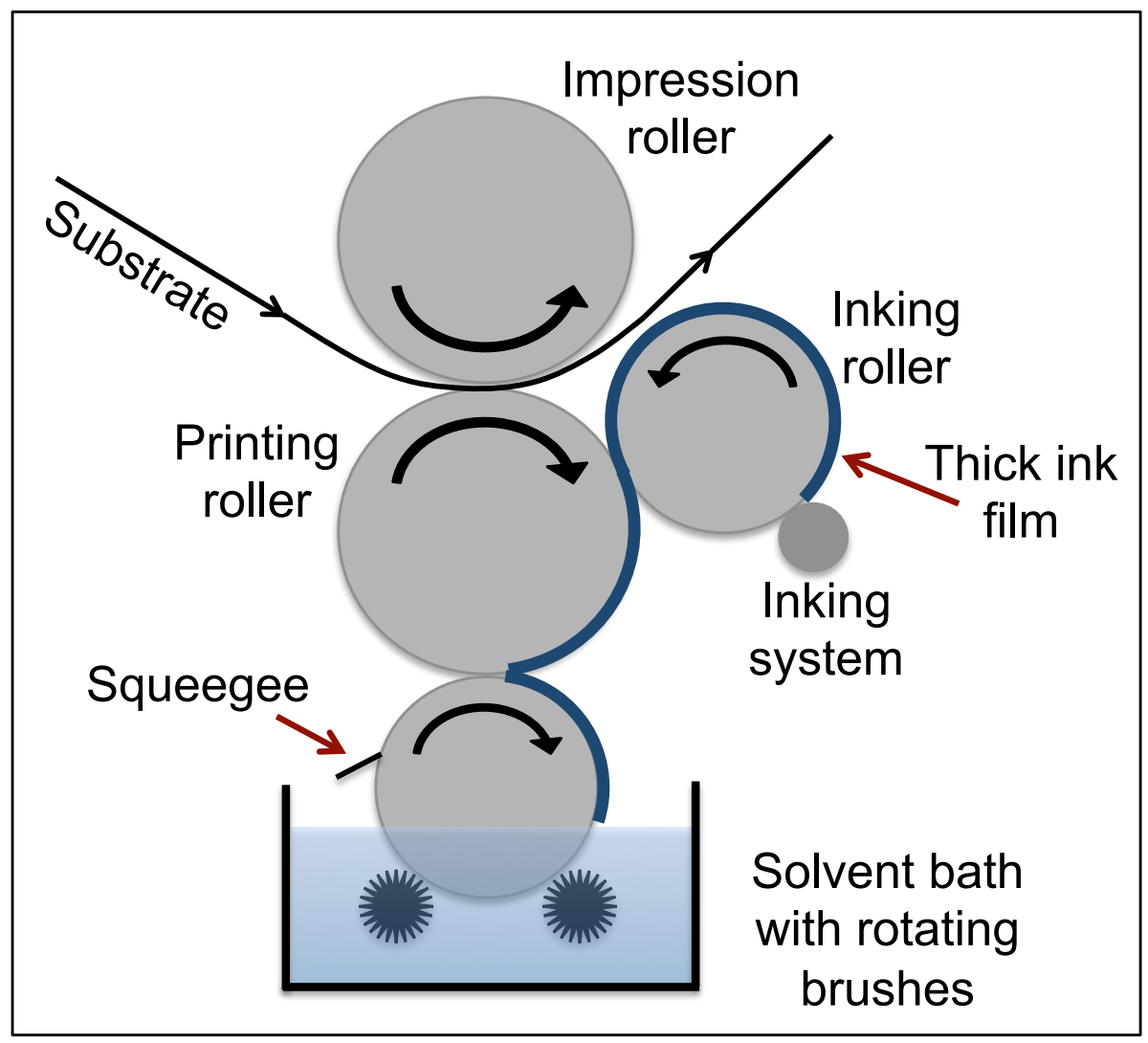

Figure 4 - Schematic of intaglio printing unit

\subsubsection{Elemental Analysis of Printing Inks}

Elemental analysis of the ink is useful for the discrimination of samples from different sources and the association of samples from the same source. The trace metals found in ink typically originate from the dyes, pigments and extenders, driers, and other additives. Although, Material Safety Data Sheets (MSDS) are available for some ink formulations, most of the components in a particular manufacturer's ink are proprietary and therefore, undisclosed. Thus, the origin of each element detected in a particular ink sample is unknown. However, a review of printing ink patents was conducted in order to determine the possible purpose of each element detected in this study (Table 6). All elements are 
accounted for, indicating that those elements are indeed relevant the characterization of inks.

Table 6 - Ink patents indicating the role of specific elements in the formulation

\begin{tabular}{|c|c|c|}
\hline $\begin{array}{c}\text { Patent } \\
\text { Number }^{76-109}\end{array}$ & Elements & Function in Ink Formulation \\
\hline US 6521032 B1 & $\mathrm{Cu}$ & Azo dye \\
\hline US 6726756 B1 & $\mathrm{Li}, \mathrm{S}$ & Conductivity agent \\
\hline US 20080096999 A1 & $\mathrm{B}$, halogens & Pigment \\
\hline US 5925178 A & $\mathrm{Al}, \mathrm{Si}$ & Stabilizer for pigmented ink \\
\hline US 8623938 B2 & $\mathrm{Na}, \mathrm{Ti}, \mathrm{K}$ & $\begin{array}{l}\text { Organic carriers, surfactants, and film } \\
\text { enhancers }\end{array}$ \\
\hline EP 2118212 A2 & $\mathrm{Mg}, \mathrm{S}$ & Color bleed control agent \\
\hline US 6616273 B1 & $\mathrm{Cu}, \mathrm{Cl}, \mathrm{S}$ & Kogation prevention agent \\
\hline EP 2630199 A1 & $\mathrm{Fe}, \mathrm{Zn}, \mathrm{Ti}, \mathrm{Al}, \mathrm{In}, \mathrm{Ce}, \mathrm{Si}$ & Oxide pigment \\
\hline US 8029611 B2 & $\begin{array}{l}\mathrm{Sn}, \mathrm{Zn}, \mathrm{Cu}, \mathrm{Ni}, \mathrm{Pb}, \mathrm{Pd}, \mathrm{Pt} \\
\mathrm{Mn}, \mathrm{V}, \mathrm{Co}, \mathrm{Fe}, \mathrm{Mg}, \mathrm{Al}, \mathrm{Ge}, \\
\mathrm{Ga}, \mathrm{Ti}, \mathrm{Si}, \mathrm{S}\end{array}$ & $\begin{array}{l}\text { Sulfonated dye salt that reduces } \\
\text { kogation }\end{array}$ \\
\hline US 20070060675 A1 & $\mathrm{Al}, \mathrm{Ti}, \mathrm{Zr}$ & Conductive cross-linked polymer \\
\hline US 6899754 B2 & $\begin{array}{l}\mathrm{Mg}, \mathrm{Ca}, \mathrm{Sr}, \mathrm{Ba}, \mathrm{Sc}, \mathrm{Y}, \mathrm{La}, \mathrm{Ti} \\
\mathrm{Zr}, \mathrm{V}, \mathrm{Cr}, \mathrm{Mn}, \mathrm{Fe}, \mathrm{Ru}, \mathrm{Co}, \\
\mathrm{Rh}, \mathrm{Ni}, \mathrm{Pd}, \mathrm{Pt}, \mathrm{Cu}, \mathrm{Au}, \mathrm{Zn}, \\
\mathrm{Al}, \mathrm{Ga}, \mathrm{In}, \mathrm{Sb}, \mathrm{Bi}, \mathrm{Ge}, \mathrm{Sn}, \mathrm{Pb}\end{array}$ & $\begin{array}{l}\text { Multivalent cation to improve optical } \\
\text { density of ink }\end{array}$ \\
\hline US 4869989 A & $\begin{array}{l}\text { W, Mo, P, V, Co, Al, Mn, Cr, } \\
\text { Ni, Co, Fe }\end{array}$ & Pigment \\
\hline US 4410617 A & $\mathrm{Cl}, \mathrm{S}, \mathrm{Ca}, \mathrm{Ba}, \mathrm{Sr}$ & Pigment \\
\hline EP 0282740 A2 & $\mathrm{Na}, \mathrm{S}$ & Binder \\
\hline EP 1467258 A2 & $\mathrm{Sn}$ & Catalyst for binder resin \\
\hline EP 2244879 A2 & $\begin{array}{l}\mathrm{Li}, \mathrm{Na}, \mathrm{K}, \mathrm{Rb}, \mathrm{Cs}, \mathrm{Ca}, \mathrm{Ba} \\
\mathrm{Sr}, \mathrm{Fe}, \mathrm{Sn}, \mathrm{Mo}, \mathrm{Nb}, \mathrm{Ta}, \mathrm{Ni} \\
\mathrm{Pd}, \mathrm{Pt}, \mathrm{Cu}, \mathrm{Ag}, \mathrm{Au}, \mathrm{Zn}, \mathrm{Cd} \\
\mathrm{Al}, \mathrm{Ga}, \mathrm{In}, \mathrm{Tl}, \mathrm{W}\end{array}$ & $\begin{array}{l}\text { Tungsten oxide compound to increase } \\
\text { heat input of NIR processes (for } \\
\text { curing and drying of inks) }\end{array}$ \\
\hline US 4898802 A & $\mathrm{B}$ & Charge enhancing salt \\
\hline US 5200289 A & $\mathrm{Ba}, \mathrm{S}$ & Surfactant for liquid toner \\
\hline US 5486443 A & $\mathrm{Fe}, \mathrm{Si}, \mathrm{Sr}, \mathrm{Ti}, \mathrm{F}, \mathrm{Al}$ & Toner particle components \\
\hline US 5506079 A & $\mathrm{Yt}, \mathrm{Fe}$ & Inorganic magnetic material \\
\hline US 5512264 A & Mo & Charge control agent \\
\hline US 5538829 A & $\mathrm{B}, \mathrm{Zn}$ & Charge enhancing additive \\
\hline US 5593807 A & $\mathrm{Na}, \mathrm{S}$ & Polyester resin \\
\hline US 5998079 A & $\mathrm{B}, \mathrm{Zn}, \mathrm{Cr}, \mathrm{Mo}, \mathrm{Co}, \mathrm{Al}, \mathrm{Fe}, \mathrm{Ni}$ & Charge control agents \\
\hline
\end{tabular}




\begin{tabular}{|c|c|c|}
\hline $\begin{array}{c}\text { Patent } \\
\text { Number }^{76-109}\end{array}$ & Elements & Function in Ink Formulation \\
\hline US 20070281233 A1 & $\mathrm{Sr}, \mathrm{Ti}$ & $\begin{array}{l}\text { Surface additive (reduces toner } \\
\text { ageing effect) }\end{array}$ \\
\hline US 6824944 B2 & $\begin{array}{l}\mathrm{Co}, \mathrm{Cl}, \mathrm{Br}, \mathrm{I}, \mathrm{F}, \mathrm{Zn}, \mathrm{Sn}, \mathrm{Pd} \\
\mathrm{Cd}, \mathrm{Sb}, \mathrm{Ni}, \mathrm{Li}, \mathrm{B}, \mathrm{K}\end{array}$ & $\begin{array}{l}\text { Catalysts and reducing agents for } \\
\text { surface modification }\end{array}$ \\
\hline US $6143457 A$ & $\mathrm{~S}, \mathrm{P}, \mathrm{Cl}, \mathrm{Br}, \mathrm{Mg}, \mathrm{Zn}$ & $\begin{array}{l}\text { Polyester resin and quaternary } \\
\text { organic surface components }\end{array}$ \\
\hline US 8080353 B2 & $\begin{array}{l}\mathrm{Be}, \mathrm{Nb}, \mathrm{Cl}, \mathrm{Br}, \mathrm{I}, \mathrm{S}, \mathrm{Ca}, \mathrm{Sr} \\
\mathrm{V}, \mathrm{Ta}, \mathrm{Cr}, \mathrm{Mo}, \mathrm{Ru}, \mathrm{Fe}, \mathrm{Mn}, \\
\mathrm{Ni}, \mathrm{Cu}, \mathrm{Zn}, \mathrm{Cd}, \mathrm{W}, \mathrm{Co}, \mathrm{Ag}, \\
\mathrm{Al}\end{array}$ & Aggregating agents \\
\hline US $6080902 \mathrm{~A}$ & $\begin{array}{l}\text { Ti, Zr, Hf, V, Nb, Ta, Cr, Mo, } \\
\text { W, Ge, Sn, S, F, Si, Cl }\end{array}$ & $\begin{array}{l}\text { Catalysts for preparing polyolefin } \\
\text { waxes }\end{array}$ \\
\hline US $5445671 \mathrm{~A}$ & $\begin{array}{l}\mathrm{Al}, \mathrm{Ti}, \mathrm{Fe}, \mathrm{Zn}, \mathrm{Cr}, \mathrm{Zn}, \mathrm{Si}, \mathrm{Mg}, \\
\mathrm{Bi}, \mathrm{Cl}\end{array}$ & Pearlescent pigments \\
\hline EP 0784086 A1 & $\mathrm{Ca}, \mathrm{Ba}, \mathrm{Sr}, \mathrm{Mn}$ & Metal for forming pigment lake \\
\hline US 8157905 B2 & $\begin{array}{l}\text { In, Ti, } \mathrm{Zr}, \mathrm{Mo}, \mathrm{Cu}, \mathrm{Cr}, \mathrm{La}, \mathrm{B}, \\
\mathrm{Nb}, \mathrm{Cl}, \mathrm{Dy}, \mathrm{Pr}, \mathrm{Sn}\end{array}$ & $\begin{array}{l}\text { IR-absorbing particles (for security } \\
\text { inks) }\end{array}$ \\
\hline US 20040040468 A1 & $\mathrm{Mn}, \mathrm{Ce}$ & Drier \\
\hline US 8807036 B2 & $\begin{array}{l}\text { V, Mn, Fe, } \mathrm{Cu}, \mathrm{Ce}, \mathrm{Ca}, \mathrm{Zr}, \mathrm{Sr} \\
\mathrm{Ba}, \mathrm{Bi}, \mathrm{Zn}, \mathrm{Sn}\end{array}$ & Salts for curing ink (driers) \\
\hline
\end{tabular}

Although SEM-EDS is a well-established technique that is accessible to most forensic laboratories, few studies report its use for the analysis of printing inks; these few studies focus exclusively on toner inks. ${ }^{17,24,110}$ Previous efforts in our research group evaluated SEM-EDS as well as LA-ICP-MS for the elemental analysis of black toners and inkjets. ${ }^{29}$ In the present study, the work was expanded to include colored toners and inkjets as well as two additional ink types: offset and intaglio. ${ }^{16}$

The application of LA-ICP-MS to document examination has been fairly recent and is not currently widely adopted. ${ }^{16,} 29,34$ Nonetheless, the technique shows excellent discrimination capabilities for the analysis of printing inks. Trejos, et al. reported $100 \%$ discrimination for a set of 27 black toner samples and $95.9 \%$ discrimination for a set of 21 black inkjet samples. ${ }^{29}$ Szynkowska, et al. used cluster analysis and principal 
component analysis to show excellent separation between toners originating from different sources. ${ }^{34}$

Like LA-ICP-MS, LIBS has also more recently been applied for the characterization of printing inks. Trejos, et al. reported $89 \%$ discrimination for a set of black toners and 94.3\% discrimination for a set of black inkjets. ${ }^{29}$ Using ANOVA with Tukey's post-hoc test, Lennard, et al reported $98.4 \%$ discrimination for a set of toners and $100 \%$ discrimination for a set of inkjets. ${ }^{111}$ Subedi, et al. reported tandem LIBS and LA-ICPMS for the analysis of a small set of toner, inkjet, offset, and intaglio samples. Although LA-ICP-MS alone offered greater than 99\% discrimination, LIBS was able to provide complementary information (on the basis of the $\mathrm{Ca}, \mathrm{Fe}, \mathrm{K}$, and $\mathrm{Si}$ signals), which improved the overall discrimination. ${ }^{35}$

\subsection{Composition, Manufacture, and Elemental Analysis of Glass}

According to the American Society for Testing Materials (ASTM), glass is defined as "an inorganic product of fusion which has been cooled to a rigid condition without crystallization."3 Glass is a valuable type of forensic evidence since it can be broken during the commission of a crime, resulting in small fragments that may be transferred to and retained by nearby persons or objects. ${ }^{3}$ A forensic analyst may be asked to examine glass to associate fragments recovered on a person or object to the scene of a crime or a victim. $^{112}$ If the recovered (questioned, Q) and control (known, K) glass are distinguishable in their measureable properties, then the control glass is eliminated as a possible source for the recovered glass. If the $\mathrm{K}$ and $\mathrm{Q}$ are indistinguishable, then the $\mathrm{K}$ cannot be eliminated as a possible source for the Q. The forensic comparison of glass 
fragments can include the measurement of color, thickness, density, refractive index, and elemental composition.

\subsubsection{Brief History of Glass}

Historically, early humans used natural glass, produced by volcanic activity, for tools. It was not until circa $4000 \mathrm{BCE}$ that humans began manufacturing glass. In Egypt, at around $1500 \mathrm{BCE}$, the first glass vessels were made by dipping a shape made of clay and sand into molten glass. Press-molded glass was developed in Alexandria (c. 400 BCE). Glass blowing was developed by the Syrians (c. 200 BCE) and popularized by the Romans. The Roman glass formulations (which produced clear, colorless glass) were lost with the fall of the Roman Empire. However, these formulations were redeveloped by Venetians in the 1200s. At around the 1600s, Venetian glass was replaced with the more durable leaded glass. ${ }^{3}$ Between the nineteenth and twenty-first century, glass compositions and manufacturing processes have evolved and become automated. The composition and manufacture of glass are discussed in more detail in the subsequent sections.

\subsubsection{Glass Composition}

Most commercial glass is composed of three types of materials: formers, fluxes, and stabilizers. Some of the most common formers, fluxes, and stabilizers are included in Table $7 .^{3}$

Glass formers are compounds that will solidify without crystallization when cooled quickly after melting. Thus the formers form the loose framework of the glass structure. 
Secondary formers do not form glass on their own, but can be combined with other formers to produce glass. Examples of secondary formers include oxides of aluminum, zirconium, tin, and titanium. Glass that is composed solely of formers is costly to manufacture because of the high temperature required to melt the glass. Therefore, fluxes are included in the glass formulation in order to lower the melting temperature. The most common fluxes are alkali oxides such as soda ash, salt cake, and potash. After addition of the alkali oxide(s), the resulting mixture melts at lower temperature and is therefore easier to manufacture. However, the mixture is chemically unstable and easily dissolves or crystalizes. Stabilizers are included in the formulation to prevent dissolution or crystallization. The most commonly used stabilizers are divalent cations such as calcium and magnesium.

Table 7 - Common formers, fluxes, and stabilizers used in glass formulations

\begin{tabular}{ccc}
\hline Formers & Fluxes & Stabilizers \\
\hline $\mathrm{SiO}_{2}$ & $\mathrm{Na}_{2} \mathrm{O}$ & $\mathrm{CaO}$ \\
$\mathrm{B}_{2} \mathrm{O}_{3}$ & $\mathrm{~K} 2 \mathrm{O}$ & $\mathrm{MgO}$ \\
$\mathrm{P}_{2} \mathrm{O}_{5}$ & $\mathrm{LiO}$ & $\mathrm{Al}_{2} \mathrm{O}_{3}$ \\
$\mathrm{GeO}_{2}$ & $\mathrm{Al}_{2} \mathrm{O}_{3}$ & $\mathrm{PbO}$ \\
$\mathrm{V}_{2} \mathrm{O}_{5}$ & $\mathrm{~B}_{2} \mathrm{O}_{3}$ & $\mathrm{SrO}$ \\
$\mathrm{As}_{2} \mathrm{O}_{3}$ & $\mathrm{Cs}_{2} \mathrm{O}$ & $\mathrm{BaO}$ \\
$\mathrm{Sb}_{2} \mathrm{O}_{5}$ & & $\mathrm{ZnO}$ \\
& & $\mathrm{ZrO}$ \\
\hline
\end{tabular}

Most of the raw materials for the production of glass are geologically derived: quartz sand $\left(\mathrm{SiO}_{2}\right)$ for silicon; limestone $\left(\mathrm{CaCO}_{3}\right)$ and dolomite $\left[\mathrm{CaMg}\left(\mathrm{CO}_{3}\right)_{2}\right]$ for calcium and magnesium; and trona $\left(\mathrm{Na}_{2} \mathrm{CO}_{3} \cdot \mathrm{NaHCO}_{3} \cdot \mathrm{H}_{2} \mathrm{O}\right)$ for sodium. Some of these natural materials can be substituted with manufactured materials such as salt cake $\left(\mathrm{Na}_{2} \mathrm{SO}_{4}\right)$ and 
cullet (recycled glass). All materials have chemical impurities that lead to slight variations in the final product.

The raw material used in the greatest quantity is quartz sand. However, few sources of quartz sand have the purity required for glassmaking. Common contaminants include the following: feldspars, $(\mathrm{K}, \mathrm{Na}) \mathrm{AlSi}_{3} \mathrm{O}_{8}-\mathrm{CaAl}_{2} \mathrm{Si}_{2} \mathrm{O}_{8}$; iron, which can color the glass and change the furnace temperature; and heavy refractory minerals (e.g., $\mathrm{Al}_{2} \mathrm{SiO}_{5}, \mathrm{FeCr}_{2} \mathrm{O}_{4}$, $\mathrm{MgAl}_{2} \mathrm{O}_{4}$ ), which can color the glass or produce "stones" (non-glassy materials) in the final product. ${ }^{3}$

Trona is processed into soda ash $\left(\mathrm{Na}_{2} \mathrm{CO}_{3}\right)$. It is the primary source of sodium and the second greatest raw material used. Secondary sources of sodium include sodium nitrate, borax, and salt cake. These secondary materials can act as fining agents, which remove gas bubbles from the molten glass during production.

Calcium and magnesium contribute to the chemical durability of glass. Magnesium also affects the viscosity and prevents crystallization. Moreover, in the production of float glass (discussed in a subsequent section), magnesium prevents the reaction of the float glass with the tin bath.

For glasses requiring aluminum, the major aluminum sources include: feldspar, kaolin, and slag (from blast furnaces). Aluminum inhibits devitrification, improves the chemical durability of soda-lime glass, and improves the shock resistance of leaded glass. ${ }^{3}$

Recycled glass, cullet, can be added to the batch mix in order to lower the melting temperature. Cullet reduces production costs by replacing more expensive raw materials and through energy savings during manufacture. Moreover, using cullet extends the life of the furnace walls used to melt the glass. The cullet must match the type of glass being 
produced; that is, recycled container glass can be used to produce containers and recycled flat glass (usually from within the same plant) can be used to produce flat glass. ${ }^{3}$

Even though glass production is an automated procedure, variations in the final product occur. The raw materials contain contaminants that vary in composition and concentration. Moreover, oxides (aluminum, zirconium, and silicon) can leach from the furnace walls to the molten glass. These small variations are measureable and useful for discriminating glass, even from the same manufacturing plant. $^{3}$

\subsubsection{Soda-lime Glass}

Soda-lime-silicate glass (also called soda-lime glass) is the most common composition of glass used for containers and flat glass and is thus also the most commonly encountered type of glass in forensics. Therefore, only this type of glass will be described in more detail. Other types of glass, not described here, include: borosilicate glass, aluminosilicate glass, lead-alkali-silicate glass, and silica glass. ${ }^{3}$

Soda-lime glass is composed mostly of quartz sand ( $72 \%)$. Sodium and potassium are added to lower the melting temperature and calcium, aluminum, and magnesium are added to improve the chemical durability. The typical composition of container and flat soda-lime glass is provided in Table $8 .^{3}$

Table 8 - Typical composition of container glass and flat glass

\begin{tabular}{cccccccccc}
\hline & $\mathrm{SiO}_{2}$ & $\mathrm{CaO}$ & $\mathrm{Na}_{2} \mathrm{O}$ & $\mathrm{K}_{2} \mathrm{O}$ & $\mathrm{Al}_{2} \mathrm{O}_{3}$ & $\mathrm{MgO}$ & $\mathrm{B}_{2} \mathrm{O}_{3}$ & $\mathrm{Fe}_{2} \mathrm{O}_{3}$ & $\mathrm{BaO}$ \\
\hline $\begin{array}{c}\text { Container } \\
\text { Glass }\end{array}$ & 72 & 10 & 13 & 0.5 & 2 & 0.3 & 0.3 & 0.05 & 0.1 \\
$\begin{array}{c}\text { Flat } \\
\text { Glass }\end{array}$ & 72 & 7 & 14 & - & 0.9 & 3 & - & 0.2 & 0.002 \\
\hline
\end{tabular}


Colored soda-lime glass is produced by adding oxides of transition metals and rare earth elements. For example, a green hue can be produced by adding minor amounts of iron and chromium oxide. Cobalt produces a blue color. Red can be produced using copper, selenium, or colloidal particles of gold. Brown is produced using colloidal particles of iron, sulfur, titanium, or combinations of those elements. For some transition elements (e.g., iron), the color produced depends on the valence state of the element in the final product.

Colorless glass is made by removing or masking the color produced by contaminants, mostly iron. The process of decolorizing involves the destruction of carbonaceous matter and the oxidation of ferrous iron $\left(\mathrm{Fe}^{2+}\right)$ to ferric iron $\left(\mathrm{Fe}^{3+}\right)$, which changes the blue-green color to a less intense yellow-green. The oxidation of carbon and iron can be accomplished with nitrates (or sulfates in neutral conditions) of sodium, potassium, and barium. Other decolorizers include arsenic, antimony, cerium, and selenium. ${ }^{3}$

\subsubsection{Glass Manufacturing}

\subsubsection{Flat Glass}

Originally, flat glass was produced by blowing a sphere of glass at the tip of a pipe. The sphere was transferred to a solid iron rod, reheated, and then opened to form a flat disc. The disc was allowed to cool and then cut into rectangular pieces. Since the disc had differences in thickness, the rectangular panes were thicker on one end than at the other end. In the 1900s, this method was replaced by a process in which the molten glass is drawn between a series of rollers. There are different roller processes with slight variations (Fourcault, Libbey Owens, and Pittsburgh processes), but the principle remains 
the same for each process. Unfortunately, the rolling method leaves striations in the glass that must be removed by polishing. The float method for the production of flat glass, implemented by Pilkington Brothers Ltd., solved the problems of the previous two methods. The process has been in general use since the 1960s. In the float process, the molten glass floes onto a bath of molten tin. Since the tin bath is denser than the glass, the glass floats on top of the tin, hence the name of the process. The thickness of the glass is controlled by the rate at which the glass moves to the cooler regions of the annealing area. In the United States, flat glass is manufactured almost exclusively by the float process. ${ }^{3}$

Flat glass is typically decolorized soda-lime glass. Flat glass may be toughened to improve its resistance to breakage. This type of glass is referred to as tempered glass. Thermally tempered glass is made by heating and rapidly cooling the surface of the glass so that the surface is in compression and the interior is in tension. Glass is stronger when it is under compression; thermally treated glass is approximately 4.5 times more resistant to breakage than non-tempered glass. Tempered glass is typically used for architectural windows or the side and rear windows of vehicles. Laminated glass is made by heatsealing a plastic film, usually polyvinyl butyral (PVB), between two panes of glass. Laminated glass is used for windshields in automobiles in order to reduce injury in the event of a vehicle collision. Tinted windows are usually made by adding color to the polymer between the two glass panes. The two glass panes can differ in color, thickness, refractive index, and elemental composition. ${ }^{3}$ 


\subsubsection{Container Glass}

For the manufacture of container glass, molten glass flows out of the furnace and is cut into sections called "gobs." The gobs are dropped into "gather cups," which transfer the gobs to molds. The molten glass takes the shape of the mold by a pressing, blowing, casting, or spinning process. Figure 5 shows an example of an early mold developed by Philip Arbogast in the late 1800s and the I.S. process developed by Ingle and Smith that is currently in use today. Both photos were taken at the Corning Museum of Glass in Corning, New York.

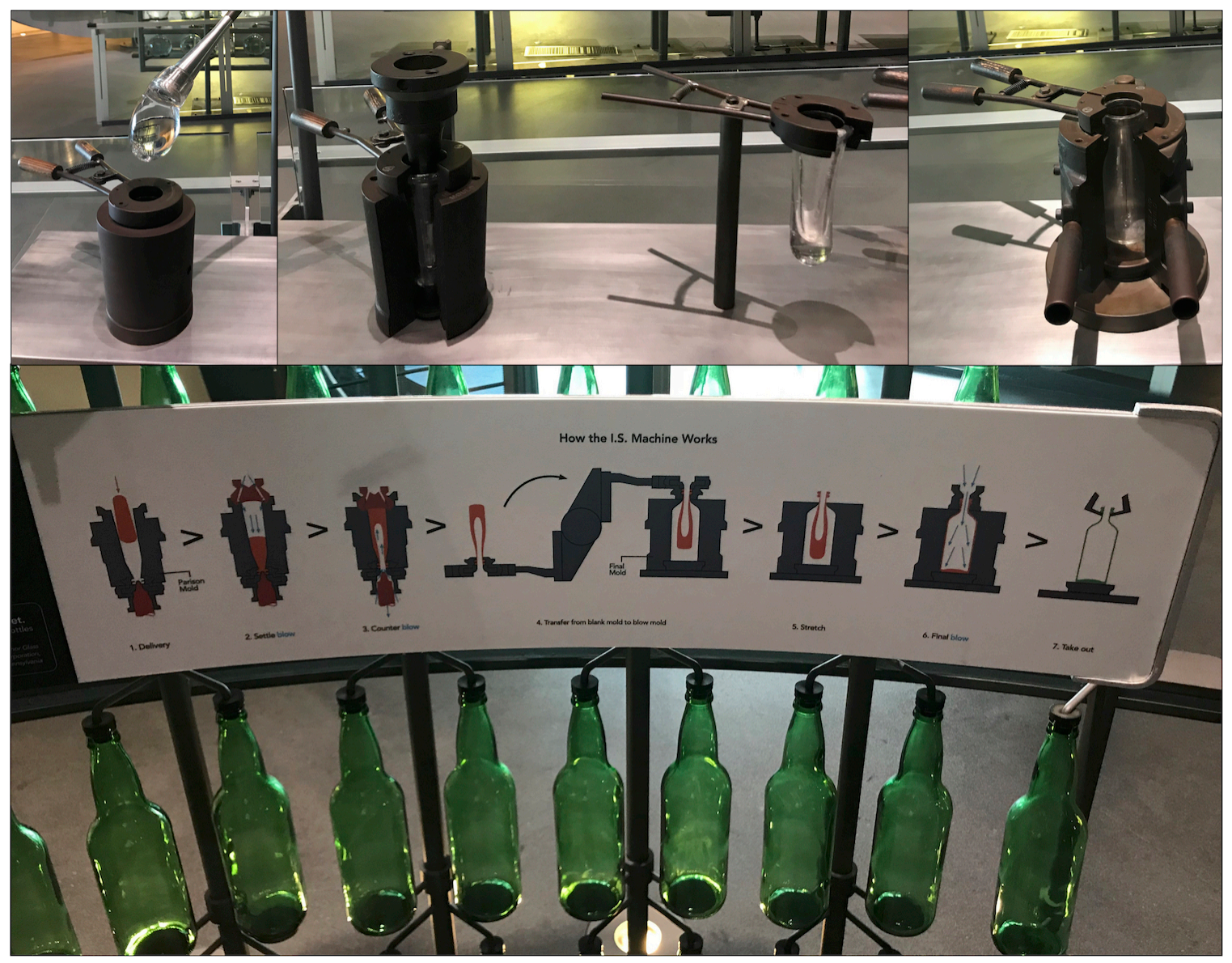

Figure 5 - Arbogast method (top) and I.S. method (bottom) of manufacturing containers 
As shown in Table 8, container glass typically has lower amounts of magnesium and greater amounts of calcium compared to flat glass. A micro-homogeneity study revealed that container glass exhibits greater within-sample heterogeneity than flat glass (automotive windshields and architectural windows) ${ }^{69}$

\subsubsection{Annealing}

Strain remains in glass when it is cooled too rapidly, resulting in a product that is prone to spontaneous breakage. Annealing is a process that reduces this strain without significantly changing the shape of the product. Glass is annealed by heating it to a temperature that is below the softening and deformation temperature; for most glasses, this temperature is between $500^{\circ} \mathrm{C}$ and $650^{\circ} \mathrm{C}$. The glass is then cooled slowly to minimize the formation of strains. Typically, the annealing process occurs in a long tunnel, called a lehr, through which the glass can move at a controlled speed. One end of the lehr is hotter than the other end, which allows the glass to cool slowly as it moves from the hot end to the cooler end. For practical reasons, only optical glasses are perfectly annealed. ${ }^{3}$

\subsubsection{Elemental Analysis of Glass}

As previously mentioned, the differences in elemental concentrations of the raw materials and/or contaminants in the glass formulation can be useful for discrimination purposes. Several analytical tools may be used to characterize the elemental profile of glass samples: Scanning Electron Microscopy-Energy Dispersive Spectroscopy (SEM-EDS), Neutron Activation Analysis (NAA), X-ray Fluorescence Spectroscopy (XRF), and 
Inductively Coupled Plasma (ICP) techniques. However, forensic labs typically employ XRF or ICP techniques. ${ }^{112}$

The Natural Isotopes and Trace Elements in Criminalistics and Environmental Forensics (NITECRIME) European Network developed a quantitative methodology for the analysis of glass fragments using LA-ICP-MS. ${ }^{73,113}$ The group also developed two new float glass standards (FGS 1 and FGS 2) that served as matrix-matched standards for the analysis of soda-lime glass using LA-ICP-MS. Trejos, et al. conducted a comprehensive study comparing $\mu \mathrm{XRF}$, solution ICP-MS, laser ablation (LA) ICP-MS, and LA-ICP-OES as part of the Elemental Analysis Working Group (EAWG). ${ }^{36,70}$ Both $\mu$ XRF and ICP-based methods performed well in terms of accuracy and precision using glass standards (NIST 612, NIST 1831, FGS 1, and FGS 2). Moreover, all participating laboratories correctly associated same-source and correctly discriminated different-source glass samples that were submitted as mock casework to each lab. As expected ICP-based techniques provided superior sensitivity; the $\mu \mathrm{XRF}$ limits of detection (LODs) were typically 2 to 3 orders of magnitude greater than those of ICP techniques. Still, the authors concluded that both $\mu \mathrm{XRF}$ and ICP-based techniques are fit-for-purpose for the forensic analysis of glass. The evaluation of the performance for several match criteria ultimately led to a standard methodology for $\mu$ XRF and LA-ICP-MS (ASTM E2926 and ASTM E2927, respectively). ${ }^{114-115}$

More recently, Laser Induced Breakdown Spectroscopy (LIBS) has been applied for the analysis of glass. ${ }^{116-120}$ Although a promising technique, LIBS is a relatively immature analytical technique compared to the well-established techniques $\mu \mathrm{XRF}$ and ICP 
techniques. Moreover, no standard methodology currently exists for the analysis of glass using LIBS.

\subsection{Fundamentals of Instrumentation}

\subsubsection{Scanning Electron Microscopy-Energy Dispersive Spectroscopy}

In Scanning Electron Microscopy (SEM), a beam of electrons is generated (usually with a tungsten filament) and focused onto the sample surface through a series of lenses. The beam penetration into the sample depends on the density and atomic number of the target; deeper penetration is seen for low density and low atomic samples. Imaging of the sample surface can be accomplished through the detection of backscattered electrons or secondary electrons. Backscattered electrons (BSE) are beam electrons that undergo elastic scattering through the sample and eventually return to the sample surface. Secondary electrons (SE) are generated by the ejection of loosely bound outer shell electrons in the sample; this occurs when the sample electrons receive enough kinetic energy from inelastic scattering of the beam electrons. SE produced by the incoming electron beam are referred to as $\mathrm{SE}_{1}$ and $\mathrm{SE}$ produced by the outgoing electron beam are referred to as $\mathrm{SE}_{2}$. Since BSE occur through elastic scattering of the high-energy beam electrons and SE occur through inelastic scattering, BSE typically have much higher energy than SE (5-50 keV versus 3-5 eV, respectively). To create an image, the electron beam is rastered along the sample surface. The beam location is controlled by changing the current strength of the scanning coils. At each position on the sample, the BSE and/or the SE ejected are detected. For display, the image is generated on a cathode ray tube (CRT), which is scanned simultaneously with the scanning of the sample surface. The 
SEM image is essentially an intensity map on the basis of the signal of the BSE and/or SE detected. ${ }^{121}$

The most commonly used detector in SEM is the combined BSE/SE Everhart-Thornley (E-T) detector. When a high-energy electron $(\sim 10 \mathrm{keV})$ strikes the scintillator material in the detector, light is emitted. The scintillator material can be a doped plastic or a crystalline compound. The emitted light is guided, via a plastic or glass rod, to a photomultiplier (PM). At the first electrode in the PM, the light is converted back to electrons. These electrons are successively amplified by a series of electrodes held at decreasing negative potentials until the final collector is reached and the electric current can be converted to the SEM image. SE (and some lower-energy BSE) do not have sufficient energy to directly excite the scintillator material in the E-T detector. However, a thin metal coating on the surface of the scintillator held at a high positive potential $(+10$ $\mathrm{kV}$ and $+12 \mathrm{kV}$ ) can sufficiently accelerate the lower-energy electrons in order to excite the scintillator. A Faraday cage that is insulated from the scintillator bias surrounds the scintillator. When the E-T detector is biased negatively (i.e., the Faraday cage has a negative bias), only BSE with trajectories directly facing the scintillator are detected; all SEs and those BSE that are not within the line-of-sight of the scintillator are rejected. When the detector is positively biased, SE and low-energy BSE are detected. The positive bias of the Faraday cage does not affect the collection of high-energy BSE that have a trajectory directly facing the scintillator. However, since few BSE directly face the scintillator, most BSE collide with the chamber walls and are converted to secondary electrons $\left(\mathrm{SE}_{3}\right)$. These $\mathrm{SE}_{3}$ are then accelerated by the positive potential toward the scintillator. Thus, a positive bias leads to an indirect detection of BSE. There are several 
other types of detectors that may be used for SEM imaging. However, these will only briefly be discussed since they are less common than the E-T detector. The "Through-the Lens" (TTL) detector is a dedicated SE detector, although BSE that are converted to $\mathrm{SE}_{3}$ are detected as well. Several dedicated BSE detectors are available: the Passive Scintillator BSE detector, the BSE-to-SE Conversion detector, and the Solid State Diode detector. $^{121}$

Apart from surface imaging, elemental analysis is possible with an Energy Dispersive Spectrometer (EDS). When the electron beam strikes the sample surface, two types of $\mathrm{x}$ ray photons can be emitted: continuum (Bremsstrahlung) and characteristic x-ray photons. Bremsstrahlung radiation occurs when the beam electrons are decelerated by the Coulombic field of the specimen atoms. The loss of energy that occurs is emitted as a photon with energy equal to: $\Delta E=h \nu$, where $h$ is Planck's constant and $\nu$ is the frequency. Bremsstrahlung radiation yields background radiation and has no relationship to the sample's elemental composition. The beam electrons can interact with the tightly bound inner shell electrons of the specimen atom causing an electron to be ejected. The atom is left in an excited state with a missing inner shell electron. The excited atom relaxes to its ground state when an outer shell electron fills the inner shell vacancy, emitting an x-ray photon in the process. Since atomic energy levels are sharply defined, the outer shell to inner shell transition results in the emission of an x-ray photon with an energy that is characteristic to a particular element. Therefore, characteristic $\mathrm{x}$-rays provide information about the elemental composition of the sample. The characteristic $\mathrm{x}$ ray photons are detected by an Energy Dispersive Spectrometer, the most common of which is the lithium-drifted silicon $\mathrm{Si}(\mathrm{Li})$ solid-state detector. ${ }^{121}$ The $\mathrm{x}$-ray photon from 
the specimen travels through a window and into a cooled reverse-bias $p$-i- $n$ ( $p$-type, intrinsic, and $n$-type) $\mathrm{Si}(\mathrm{Li})$ crystal. $N$-type semiconductors have excess electrons and act as negative charge carriers. $P$-type semiconductors have a shortage of electrons (creating vacancies called "holes") and act as positive charge carriers. ${ }^{75}$ An intrinsic semiconductor (e.g., pure silicon) has neither excess electrons nor holes. Intrinsic semiconductors do not conduct current in an applied electric field unless they absorb energy causing electrons to be ejected into the conduction band; this feature is important for the detection of radiation such as X-ray photons. ${ }^{121}$ However, sufficiently pure silicon capable of achieving intrinsic properties is difficult to obtain. Thus, lithium (an $n$-type dopant) is added to the surface of $p$-type silicon, and diffuses into the crystal forming a thin $p-n$ junction. A reverse bias applied to the $p-n$ junction at elevated temperatures enlarges this intrinsic region. Absorption of the $\mathrm{x}$-ray photon by a silicon atom in the intrinsic layer of the $\mathrm{Si}(\mathrm{Li})$ crystal results in the ejection of a photoelectron with energy equal to $h \nu-E_{c}$ (for silicon, $E_{c}=$ $1.84 \mathrm{keV})$. The ejected photoelectron releases most of its energy to the formation of electron-hole pairs, which are swept away by the applied bias to create a charge pulse. The charge pulse is converted to a voltage pulse by a charge-to-voltage converter (preamplifier). Each voltage pulse is proportional to the energy of the incoming x-ray photon from the specimen. The voltage pulse is amplified further using a linear amplifier and passed to a computer x-ray analyzer. Finally, the data are displayed as a histogram of intensity by voltage. ${ }^{121}$

The basic components in SEM-EDS are shown in Figure 6. SEM-EDS is a surface technique, penetrating no more than a few microns into the sample, which is advantageous for ink analysis since it minimizes the contribution from the paper 
substrate. On the other hand, the laser ablation methods subsequently described are considered to be bulk-sampling techniques.

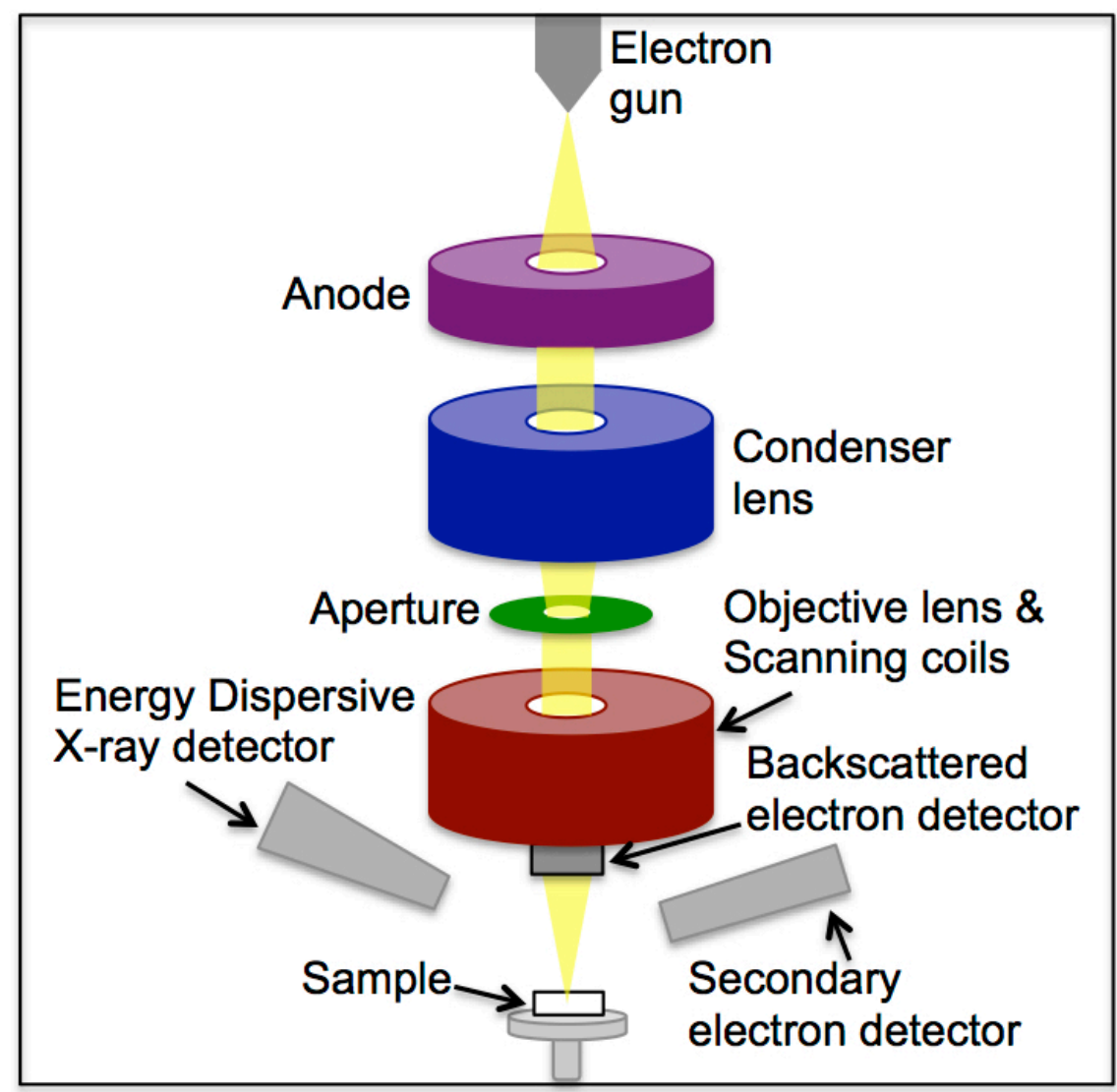

Figure 6 - Schematic of SEM-EDS

\subsubsection{Laser Ablation-Inductively Coupled Plasma-Mass Spectrometry}

Laser Ablation-Inductively Coupled Plasma-Mass Spectrometry (LA-ICP-MS) is a wellestablished analytical technique for the elemental characterization of solid samples. Figure 7 provides a schematic with the basic LA-ICP-MS components.

In LA-ICP-MS, a pulsed laser is used to remove (ablate) small quantities of the sample (typically microgram quantities). A laser (Light Amplification by Stimulated Emission of Radiation) is an optical transducer that converts energy into intense light. Lasers have 
three main components: an energy pumping source, a lasing medium, and an optical cavity or resonator. For pulsed lasers, the pump source is typically a flash lamp. The energy source is used to create a buildup of excited state atoms (or molecules) within the lasing medium; this is known as "pumping" and it results in a "population inversion." The ratio of the populations for two energy levels of a particular atom is given by the Boltzmann relationship:

$$
\frac{N_{2}}{N_{1}}=\frac{g_{2}}{g_{1}} \times e^{\left[\left(E_{1}-E_{2}\right) / k T\right]}
$$

$N_{1}$ is the population of lower energy state $\left(E_{1}\right), N_{2}$ is the population of the upper energy state $\left(E_{2}\right), k$ is the Boltzmann constant, $T$ is the temperature, $g_{l}$ is the statistical weight of the lower energy level, and $g_{2}$ is the statistical weight of the upper energy level. Under thermodynamic equilibrium, there is usually a larger population in the lower energy level: $N_{l}>N_{2}$. When population inversion occurs $\left(N_{2}>N_{l}\right)$ the lasing material acts as a light amplifier. The optical resonator refers to the rod-shaped lasing material that is closed off with two mirrors, one of which is semitransparent. When a few atoms emit light by spontaneous emission (i.e., emission without an external energy source), the emitted photons can stimulate emission from other excited atoms. When the stimulated emission occurs between the two mirrors, it is reflected back and forth through the lasing medium, causing further amplification of the emission; in this stage, the light is said to be resonant with the cavity. A buildup of energy at a particular wavelength and resonant frequency occurs within the cavity, resulting in monochromaticity. There are many different types of lasers: dye lasers (e.g., polyphenyl 2, courmarin 102), gas lasers (e.g., carbon dioxide, excimer), semiconductor lasers (e.g., ZnSe, GaAs), and solid-state lasers 
(Ruby, Nd:YAG). Since the neodymium-doped yttrium aluminum garnet (Nd:YAG) laser is used in the present study, only this laser will be discussed in more detail. The neodymium ions are responsible for the laser action of the Nd:YAG laser. More specifically, excited state $\mathrm{Nd}$ ions decay to a metastable level, which leads to a population inversion between the ${ }^{4} \mathrm{~F}_{3 / 2}$ and ${ }^{4} \mathrm{I}_{11 / 2}$ states. The fundamental wavelength for the Nd:YAG laser occurs at 1064 nanometers (nm); however, other wavelengths can be obtained by harmonic generation through the use of crystals (e.g., lithium niobate, $\beta$ barium borate). The two wavelengths used in this study are $213 \mathrm{~nm}$ obtained by fifth harmonic generation $(1064 \div 5=212.8)$ and $266 \mathrm{~nm}$ obtained by fourth harmonic generation $(1064 \div 4=266)$.

When a laser pulse strikes a solid sample, heating and melting occurs at the surface of the sample. The change in temperature at the sample surface can be approximated by:

$$
\Delta T \approx(1-R) I_{0} \frac{\sqrt{\kappa \tau}}{K}
$$

In the equation above, $I_{0}$ is the laser power flux at the sample surface and $R$ is the reflectivity; $\kappa, \tau$, and $K$ are the thermal diffusivity, pulse duration, and thermal conductivity of the laser, respectively. When the temperature rises sufficiently, vaporization occurs. Ionization of the expanding vapor, which gives rise to a micro plasma, occurs via inverse bremsstrahlung collisions. Absorption of laser radiation also occurs at this point, increasing ionization in the micro plasma. ${ }^{122}$ In conjunction with the formation of a vapor plume, when the laser strikes the sample surface, solid particulates are ejected, forming a solid aerosol. In LA-ICP-MS, these particles are transported via a carrier gas (usually helium) to the secondary excitation/ionization source. ${ }^{123}$ 
Once the particulates enter the inductively coupled plasma (ICP), the sample is vaporized, atomized, and excited/ionized. The plasma support gas used for ICP-MS is typically argon since, because of its relatively high first ionization potential, it is capable of ionizing most elements in the periodic table for mass spectrometric analysis. The argon plasma is initiated by seeding a few electrons, generated by a spark using a Tesla coil or piezoelectric starter, into the support gas. These seed electrons collide with and ionize the neutral argon atoms:

$$
e+\mathrm{Ar} \rightarrow \mathrm{Ar}^{+}+2 e
$$

Through radiative recombination, argon atoms may recombine with electrons to yield excited argon atoms $\left(\mathrm{Ar}^{*}\right)$ :

$$
e+\mathrm{Ar}^{+} \rightarrow \mathrm{Ar}^{*}+h v
$$

Radiative recombination leads to significant background emission in the ultraviolet region. Additionally, Bremsstrahlung radiation, described in the previous section, occurs in the visible region. ${ }^{124}$ Once the plasma is initiated, it is sustained by an electromagnetic $\mathrm{RF}$ field (via an induction coil connected to a radiofrequency generator) as well as a constant flow of argon gas. ${ }^{123}$ Temperatures in the ICP are typically in the range of $8,000-10,000 \mathrm{~K}^{125}$

When the analyte, denoted as $M$, enters the plasma, various ionization and excitation processes can occur (Table 9). ${ }^{124}$ There are three main ionization processes: chargetransfer ionization, in which an argon ion transfers energy to the analyte atom resulting in an excited analyte ion $\left(\mathrm{M}^{+*}\right)$ and a neutral Ar atom; electron impact ionization, in which a fast moving electron (i.e., high kinetic energy) transfers energy to the analyte atom resulting in an analyte ion and two electrons with lower kinetic energy; and Penning 
ionization, in which a metastable argon atom $\left(\mathrm{Ar}^{\mathrm{m}}\right)$ transfers energy to the analyte resulting in an excited analyte ion. There are two main excitation processes: electron impact excitation, in which an electron transfers energy to the analyte, resulting in an excited analyte atom; and ion-electron radiative recombination, in which an electron excites an analyte ion. In the case of ICP-MS, the analyte ions, not neutral atoms, are of interest.

Table 9 - Excitation and ionization processes in the inductively coupled plasma

\begin{tabular}{lc}
\hline \multicolumn{1}{c}{ Process Name } & Process \\
\hline Charge-transfer ionization & $\mathrm{Ar}^{+}+\mathrm{M} \rightarrow \mathrm{M}^{+*}+\mathrm{Ar}$ \\
Electron impact ionization & $e_{(\mathrm{fast})}+\mathrm{M} \rightarrow \mathrm{M}^{+}+2 e_{(\mathrm{slow})}$ \\
Penning ionization & $\mathrm{Ar}^{\mathrm{m}}+\mathrm{M} \rightarrow \mathrm{M}^{+*}+\mathrm{Ar}$ \\
Electron impact excitation & $e+\mathrm{M} \rightarrow \mathrm{M}^{*}+e$ \\
Ion-electron radiative recombination & $\mathrm{M}^{+}+e \rightarrow \mathrm{M}^{+*}+h v$ \\
\hline
\end{tabular}

The positive ions generated in the plasma are guided to the mass spectrometer inlet and separated according to their mass-to-charge $(\mathrm{m} / \mathrm{z})$ ratio. There are several different types of mass analyzers: quadrupole, ion trap, orbitrap, time-of-flight, magnetic and electromagnetic, and ion cyclotron resonance (ICR). ${ }^{126}$ The ICP-MS instrumentation used in this study utilizes a quadrupole mass spectrometer. Most commercial quadrupoles operate with a resolving power capable of unit mass resolution $(\mathrm{R}=300) .{ }^{123}$ The quadrupole mass analyzer consists of four circular or hyperbolic rods that are arranged in parallel (Figure 7). A direct current potential $(U)$ and a radio frequency alternating current potential $(V \cos (\omega t))$ are applied to pairs of rods. ${ }^{123}$ Two opposite rods have a combined electric potential equal to: $U+V \cos (\omega t)$. The other two opposing rods have a combined electric potential equal to: $-U-V \cos (\omega t)$. Thus, two rods are positively charged 
and the other two rods are negatively charged; moreover, the two pairs of rods are $180^{\circ}$ out of phase. As the electric potential applied to the rods varies (keeping $U / V$ constant), an electromagnetic field is created allowing ions of a particular $\mathrm{m} / \mathrm{z}$ to travel through the rods (the $\mathrm{z}$ axis) in a spiral trajectory; ions that are too light or too heavy will be deflected and will collide with the rods.

The final stages in ICP-MS analysis are detection, amplification, and signal processing. There are several types of detectors: photographic plates, Faraday cups, electron multiplier tubes (EMT), and electro-optical ion detectors. The EMT ion detector is the most widely used detector. In this detector, the ions from the mass analyzer (quadrupole) are accelerated towards a conversion dynode held at a potential between -3 and -30 kilovolts $(\mathrm{kV}) .{ }^{126}$ When positive ions strike the negative conversion dynode, which is the case for ICP-MS, secondary particles are generated: negative ions and electrons. The secondary particles are converted to electrons at the first dynode after which the electrons are amplified by a cascade effect. There are two types of electron multipliers: discrete and continuous dynode type. The discrete dynode type consists of a series of 12 to 20 dynodes, which are held at deceasing negative potentials by a chain of resistors. The secondary particles from the conversion dynode strike the first dynode causing the emission of secondary electrons. The secondary electrons are then accelerated to the second dynode, held at a less negative potential, which causes the emission of more electrons. The process continues so that with each successive dynode, an amplification of electrons occurs. The continuous dynode electron multipliers works by the same principle as the discrete dynode type. However, rather than using a series of individual dynodes, a single continuous dynode is used. There are two types of continuous electron multipliers: 
the channeltron and the microchannel plate. The channeltron consists of a curved tube made of lead-doped glass. A voltage, applied to the two ends of the tube, produces a continuous accelerating field along the tube's length. A microchannel plate consists of a plate with parallel cylindrical channels. The input side of the plate is held at a more negative voltage compared to the output side of the plate. In EMT detectors, the final flow of electrons creates an electric current that is enhanced via electronic amplification and an analogue-to-digital converter digitizes the electrical signal from the detector. Finally, a computer can then present the digital data in a compatible format. ${ }^{126}$

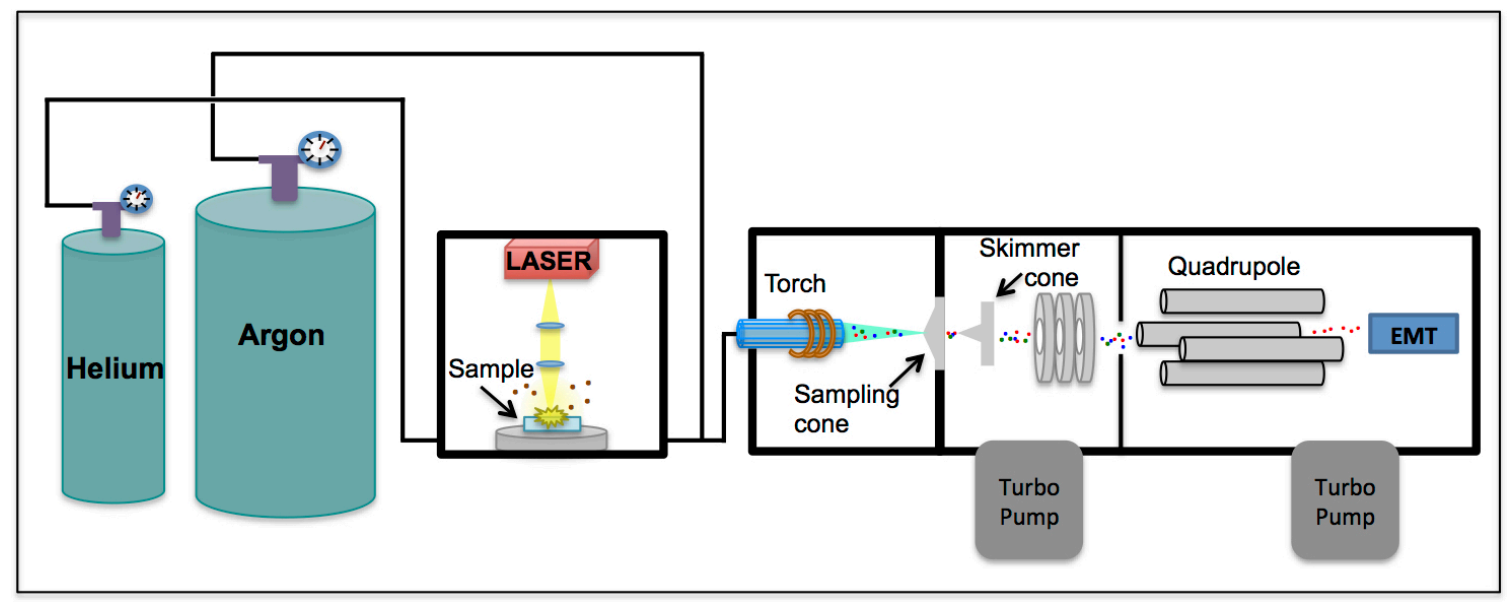

Figure 7 - Schematic of LA-ICP-MS

\subsubsection{Laser Induced Breakdown Spectroscopy}

In Laser Induced Breakdown Spectroscopy (LIBS), like in LA-ICP-MS, a pulsed laser is fired at the sample. Thus, the laser-sample interaction described for LA-ICP-MS applies to LIBS as well. However, in LIBS the micro plasma produced on the sample surface is of interest; that is, the ejected particles are not transported to a secondary 
excitation/ionization source. A schematic of the basic components in a LIBS system is shown in Figure 8.

Early in the LIBS plasma formation, the radiation is dominated by Bremsstrahlung and recombination radiation. ${ }^{127}$ To reduce the background radiation, LIBS analyses are carried out using time-resolved detection. Within the plasma, excited analyte ions, atoms, and molecules eventually revert back to their ground state, emitting radiation that is characteristic to a particular element. The emission from ions occurs first, followed by atoms, and lastly molecules. ${ }^{127}$

Typical LIBS plasmas are characterized by a dominance of electron impact processes and are thus assumed to be under Local Thermodynamic Equilibrium (LTE). ${ }^{122,}{ }^{128}$ Under LTE, the collision processes are more important than the radiative processes and for every point in the plasma, it is possible to find a temperature parameter that satisfies the Boltzmann, Saha, and Maxwell distributions. Therefore, the electronic temperature and the electron density can be used to describe the plasma characteristics. McWhirter proposed a criterion to test the LTE assumption: $\mathrm{n}_{\mathrm{e}} \geq 1.6 \times 10^{12 \times} \mathrm{T}^{1 / 2} \times \Delta \mathrm{E}^{3}$ where $n_{e}$ is the electron density, $T$ is the temperature (in Kelvin), and $\Delta E$ (in $\mathrm{eV}$ ) is the energy difference between the transition levels. Typically, the inner region of the plasma is more likely to satisfy this criterion, while the outer, cooler region of the plasma is more likely to deviate from LTE (due to the lower electron density in the cooler plasma regions). ${ }^{129}$ For the determination of electron density, the broadening of emission lines due to the Stark effect is most commonly used. The method assumes that Stark (pressure) broadening is the dominant broadening mechanism rather than other mechanisms such as Doppler (radiative) broadening. ${ }^{128,} 130$ Lines that are dominated by Stark broadening 
exhibit a Lorentzian profile and can be fitted with the following equation to determine the full width at half maximum (FWHM):

$$
y=y_{0}+\left(\frac{2 A}{\pi}\right)\left[\frac{w}{4\left(x-x_{c}\right)^{2}}+w^{2}\right]
$$

In the equation above, $A$ is the area, and $w$ is the FWHM. The FWHM must then be corrected using a monochromatic light source (e.g., laser or calibration lamp) in order to account for broadening due to the spectrometer, rather than collisional processes. The FWHM can then be used to determine the electron density using the following relationship:

$$
\Delta \lambda_{F W H M} \approx 2 \times 10^{-16} w n_{e}
$$

In this case the $w$ is the electron impact parameter, which can be found in the literature, and $\Delta \lambda_{F W H M}$ is the FWHM calculated using the Lorentzian fit. ${ }^{131}$

There are several methods used to determine the temperature of a plasma. One method involves the use of the following equation:

$$
I=\left(\frac{h c N_{0} g A}{4 \pi \lambda Z}\right) e^{(-E / k T)}
$$

$I$ is the spectral line radiant intensity, $h$ is Planck's constant, $A$ is the Einstein coefficient, $N_{0}$ is the total species population, $g$ is the statistical weight, $c$ is the speed of light, $\lambda$ is the wavelength of the selected line, $Z$ is the partition function, $E$ is the energy of the upper level, $k$ is the Boltzmann constant, and $T$ is the temperature. The equation above can be rearranged to the following:

$$
\ln \left(\frac{I \lambda}{g A}\right)=\frac{-E}{k T}-\ln \left(\frac{4 \pi Z}{h c N_{0}}\right)
$$


This form of the equation leads to a linear relationship if a Boltzmann distribution is present. ${ }^{130}$ Plotting several lines allows the determination of the temperature from the slope (which is equal to $-1 / k T$ ) of the linear fitting. The Boltzmann plot is limited to lines that have the same ionization state; that is, all lines must be atomic emission lines or all lines must be ionic emission lines with the same charge. An alternative to the Boltzmann plot is the Saha-Boltzmann plot, given by the following linear equation:

$$
\ln \left(\frac{I_{i j}^{I I} A_{m n}^{I} g_{m}^{I}}{I_{m n}^{I} A_{i j}^{I I} g_{i}^{I I}}\right)=\ln \left(\frac{2\left(2 \pi m_{e} k T\right)^{3 / 2}}{n_{e} h^{3}}\right)-\frac{\left(E_{i o n}-\Delta E_{i o n}+E_{i}^{I I}-E_{m}^{I}\right)}{k T}
$$

The superscript $I$ denotes the atomic parameters while the superscript $I I$ denotes the ionic parameters; thus both atomic and ionic emission lines may be used to generate a SahaBoltzmann plot. $E_{\text {ion }}$ is the ionization potential of the element, $\Delta E_{\text {ion }}$ is the lowering correction parameter, which is typically on the order of $0.1 \mathrm{eV}$, and $m_{e}$ is the electron mass; all other parameters have been defined above for the Boltzmann plot. ${ }^{127,}{ }^{132}$ Apart from the capability of using both atomic and ionic emission lines, another advantage of the Saha-Boltzmann is the fact that the electron density, $n_{e}$, can be calculated using the yintercept without the need to correct for spectrometer broadening. Based on the literature, typical plasma temperatures for LIBS are $\sim 10,000-12,000$ Kelvin, depending on the gate delay. ${ }^{130}$

The emission radiation from the micro plasma is focused onto a fiber optic with the use of collection lenses. This collected light is then dispersed using a prism or grating. Various materials can be used to construct a prism including: barium flint, quartz, and borosilicate crown glass. In a prism, dispersion occurs because of the wavelength 
dependence of the material's refractive index. The variation of the deviation from the incident beam is the angular dispersion, $D_{a}$, and is defined as:

$$
D_{a}=\frac{d \eta}{d \lambda} \frac{d \theta}{d \eta}
$$

The term $d \eta / d \lambda$, known as the dispersion of the prism material, describes the refractive index $(\eta)$ variation with wavelength $(\lambda)$. The term $d \theta / d \eta$ is a geometric factor that depends on the shape and size of the prism; $\theta$ is the angle between the refracted ray and the un-deviated incident beam. The geometric factor only slightly varies with wavelength and thus the angular dispersion is primarily influenced by the term $d \eta / d \lambda .{ }^{133}$ A diffraction grating is a planar or concave plate with closely spaced grooves. When polychromatic light strikes the grating, various wavelengths are dispersed because each wavelength undergoes constructive interference at a different diffraction angle. The angular dispersion of a grating is given by:

$$
D_{a}=\frac{\sin \alpha+\sin \beta}{\lambda \cos \beta}
$$

In the equation above, $\alpha$ is the angle of incidence and $\beta$ is the angle of diffraction, both measured with respect to the grating normal. ${ }^{133}$

Once the incoming radiation is dispersed, the diffracted light is detected, usually with a photodiode array (PDA) or a charge-coupled device (CCD) array. ${ }^{130-131}$ A PDA is a linear array of hundreds or thousands of discrete photodiodes ( $p-n$, or PIN, junctions) on an integrated circuit. Incident light creates electron-hole pairs and the electrons move toward the nearest PIN junction. The diodes are sequentially read by measuring the amount of charge that must be added to each diode to achieve a neutral charge. ${ }^{130}$ A charge coupled device (CCD) is an integrated circuit that forms light sensitive elements called pixels 
arranged in a two-dimensional array. The CCD functions similarly to the PDA in that it integrates the incident light. However, unlike the PDA, the CCD also measures the vertical distribution of the light. The CCD is read by sequentially shifting the electrons in each row down to the shift register. The electrons on the shift register are then moved, pixel by pixel, to an amplifier after which the charge is read. ${ }^{75}$ Once the pixel information in the shift register is read, the next row is shifted downward and so on.

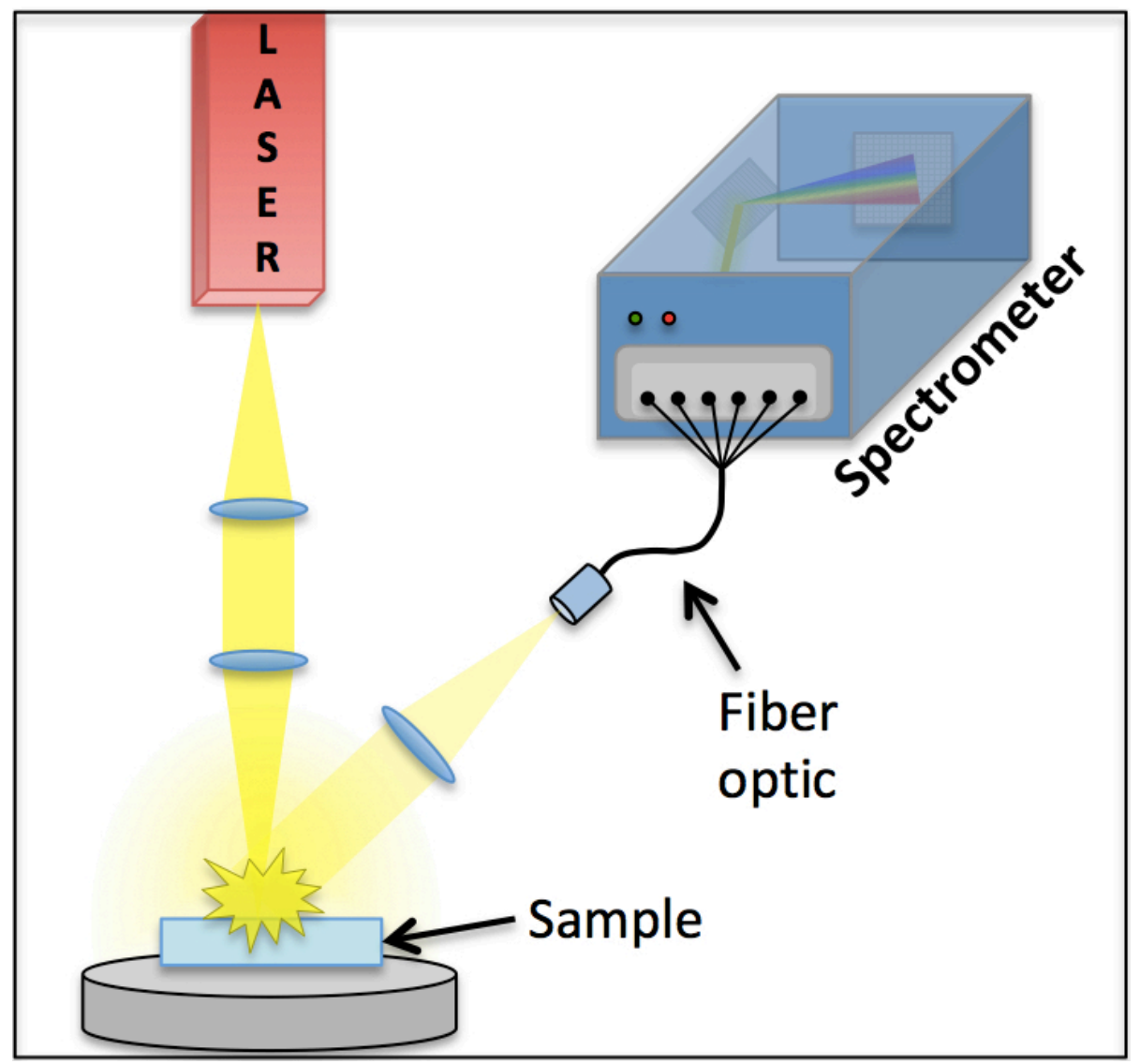

Figure 8 - Schematic of LIBS 


\subsection{Interpretation of Forensic Evidence}

\subsubsection{Match Criterion}

The performance of match criteria using elemental data in order to distinguish differentsource or associate same-source glass fragments has been extensively researched. ${ }^{36-38,114-}$ 115, 134 Koons and Buscaglia analyzed 209 glass fragments using Inductively Coupled Plasma-Atomic Emission Spectroscopy (ICP-AES) ${ }^{38}$ The equal-variance T-test (at 95\% confidence and with the Bonferroni correction) led to 2 false inclusions, resulting in a low false inclusion rate of $0.009 \%$. When using the unequal variance T-test, as opposed to equal variance, a higher false inclusion rate of $0.055 \%$ was obtained (12 falsely included pairs). On the other hand, using range overlap resulted in no false inclusions. The authors concluded that either tool (i.e., Bonferroni-corrected T-test or range overlap) is appropriate for the comparison of glass fragments. However, it should be noted that same-source comparisons were not included in this study; thus, the false exclusion rate of each statistical tool is not investigated.

A larger study, the Elemental Analysis Working Group (EAWG), investigated the performance of several match criteria using elemental data collected using X-ray Fluorescence Spectroscopy (XRF) and ICP techniques (ICP-MS, LA-ICP-MS, ICPAES). ${ }^{70}$ Mock casework samples were sent to each of the 9 XRF labs and 7 ICP labs as part of an inter-laboratory study. Each lab was asked to analyze the glass fragments they received and compare the data using the following match criteria: range overlap, T-test ( $99 \%$ confidence and $95 \%$ confidence with and without the Bonferroni correction), Hotellings $\mathrm{T}^{2}$, and $\pm 2,3,4,5$, and 6 standard deviations (SD). For pairwise comparisons using XRF data, range overlap and $\pm 3 \mathrm{SD}$ offered the best compromise between the false 
exclusion and false inclusion rates: $\leq 19 \%$ and $\leq 27 \%$ respectively. For ICP methods, a modified $\pm 4 \mathrm{SD}$, using a minimum SD equal to $3 \%$ of the average, performed best $(\leq$ $28 \%$ false exclusion rate and $\leq 5 \%$ false inclusion rate). The results of the EAWG study ultimately led to two standard methodologies: one for the analysis of glass using XRF (ASTM E2926) and the other for the analysis of glass using LA-ICP-MS (ASTM E2927). ${ }^{114-115}$

A separate European Working Group, the Natural Isotopes and Trace Elements in Criminalistics and Environmental Forensics (NITECRIME), developed a quantitative methodology for the analysis of glass fragments using solution LA-ICP-MS. ${ }^{73,113}$ Using the quantitative method developed, Weis et al. investigated a match criterion that takes inter-day variation into account. Two datasets were used to calculate the false exclusion and false inclusion rate, respectively: a single glass pane analyzed 44 times (6 replicates each) and a set of 62 different-source float glass samples. A control sample (DGG 1) was analyzed 90 times and the overall relative standard deviation (RSD) was calculated for each element, which was then used as a "fixed relative standard deviation" (FRSD) for pairwise comparisons. The comparison interval for the known sample was defined by an upper limit of the known average $\times(1+n \times$ FRSD $)$ and a lower limit of the known average $\div(1+n \times$ FRSD $)$. The $n$ indicates the sigma value used; sigma values of 1-6, 8 , 10,15 , and 20 were tested to determine the best compromise between the false exclusion (Type I) and false inclusion (Type II) rate. The 4-sigma match criterion performed best, with a Type I error of $14.83 \%$ and no false inclusions. Although casework samples are typically analyzed on the same day, accounting for inter-day variation is beneficial in order to establish a random match probability or a frequency. In the former case, the false 
inclusion rate for all possible comparisons using a glass database is reported; the random match probability gives an indication of the probability of coincidental "matches" between glass fragments of different origin. In the latter case, the questioned sample from casework is compared to all samples in a database and the number of "matches" is reported; the frequency provides an estimation of the rarity of a particular elemental profile (i.e., that of the questioned sample).

\subsubsection{Likelihood Ratio}

Several criticisms regarding the frequentist approach (i.e., match criterion) have been reported. First, it suffers from the "fall off the cliff" effect, in which a small change in the significance value, $p$, leads to a drastic change in the interpretation of the data. The "fall off the cliff" effect occurs when the average of the questioned sample lies close to the cut-off established by the known sample's comparison interval. For example, if the known comparison interval for one element was $5-10$ parts per million (ppm) and the average of the questioned sample was $9.99 \mathrm{ppm}$, the $\mathrm{K}$ and Q would be considered indistinguishable; but if the Q average was slightly higher (e.g., $10.01 \mathrm{ppm}$ ), the $\mathrm{K}$ and $\mathrm{Q}$ would be distinguishable. A second disadvantage of the frequentist approach is that it does not take the rarity of the elemental profile into account. This second drawback may be overcome by using a glass database to calculate a frequency; however, the use of a database to generate a frequency does not eliminate the "fall off the cliff" effect. Finally, the frequentist approach answers the "pre-data" rather than the "post-data" question. The former answers the question "what is the probability of a match if I carry out this 
procedure." The latter answers the question that the court is interested in: "how much does this evidence increase the likelihood that the suspect is guilty." $39-42$

There are numerous methods for calculating the likelihood ratio. The simplest approach is to calculate a frequency, $f=n \div N ; n$ is the number of times the questioned sample "matches" a sample in the database (including the known sample) and $N$ is the total number of samples in the database. The likelihood ratio (LR) is often estimated as the reciprocal of the frequency: $\mathrm{LR}=1 \div f=N \div n$. However, the denominator of the LR should evaluate the number of alternative sources and should therefore be estimated as: LR $=N \div(n-1) .{ }^{135}$ The frequency approach is only used when the known $(\mathrm{K})$ and questioned (Q) sample are found to be indistinguishable; if the two samples are distinguishable, then the $\mathrm{K}$ and $\mathrm{Q}$ are excluded and no LR is calculated. An advantage of this approach is its simplicity. Nevertheless, since the frequency approach uses a match criterion for pairwise comparisons, it still suffers from the "fall off the cliff" effect.

Bolck et al. compared a score-based and a feature-based method for the calculation of a likelihood ratio using the concentration of 15 impurities detected in ecstasy $(3,4-$ methylenedioxy-N-methyelamphetamine, MDMA) tablets. ${ }^{56}$ In the score-based approach, a similarity score (e.g., Manhattan distance, Euclidean distance, Pearson correlation coefficient) is calculated for all same-source and different-source pairwise comparisons. In this study, the Manhattan distance performed best and was therefore selected for pairwise comparisons. The same-source and different-source distributions are then fitted with a probability density function. In this case, the Weibull distribution provided the best fit. The LR is then computed by calculating the score (Manhattan distance) between the $\mathrm{K}$ and Q; the numerator of the LR is given by the probability of that score using the 
same-source distribution and the denominator of the LR is given by the probability of that score using the different-source distribution. In the feature-based approach, the original multivariate information (e.g., concentration of MDMA impurities) is used rather than a univariate similarity score. The same-source distribution is modeled by a multivariate normal distribution and the different-source distribution is modeled by a Kernel Density Estimation (KDE), which does not assume normality. Score-based approaches have the advantage of simplicity compared to the feature-based approach. However, there is a loss of information since the original variables are reduced to a univariate score. Thus, the magnitude of the LR depends on the similarity of the profiles and not the rarity of the profiles' features (concentration of impurities). Moreover, the score-based models can potentially result in reduced discrimination, though this was not the case in the Bolck et al. study. On the other hand, feature-based approaches make use of the original features and thus the LR accounts for the rarity of each individual feature. Overall, the featurebased approach provides larger absolute LR values, resulting in greater evidential strength.

The feature-based LR approach used in Bolck et al. was originally proposed by Aitken and Lucy and is referred to as the Multivariate Kernel (MVK) model. ${ }^{40}$ The MVK model accounts for two levels of variation: the within-source variation (multivariate normal) and the between-source variation (KDE). An alternative approach, the Multivariate Normal model (MVN), assumes multivariate normality for both the within-source and betweensource variation. The authors recommend the MVK model but state that if the betweensource distribution is well represented by a multivariate normal distribution, then the MVN model may perform as well as the MVK model. The LR calculation using the 
MVK or MVN model can be implemented using the freely available $\mathrm{R}$ packages: "comparison," "nnls," and "isotone."136-138 The numerator of the LR using the MVK model is given by the following equation:

$$
\begin{aligned}
f_{0}\left(\overline{\mathbf{y}}_{1}, \overline{\mathbf{y}}_{2} \mid U, C\right) & =(2 \pi)^{-p}\left|D_{1}\right|^{-\frac{1}{2}}\left|D_{2}\right|^{-\frac{1}{2}}|C|^{-\frac{1}{2}}\left(m h^{p}\right)^{-1}\left|D_{1}^{-1}+D_{2}^{-1}+\left(h^{2} C\right)^{-1}\right|^{-\frac{1}{2}} \\
& \times \exp \left\{-\frac{1}{2}\left(\overline{\mathbf{y}}_{1}-\overline{\mathbf{y}}_{2}\right)^{\mathrm{T}}\left(D_{1}+D_{2}\right)^{-1}\left(\overline{\mathbf{y}}_{1}-\overline{\mathbf{y}}_{2}\right)\right\} \\
& \times \sum_{i=1}^{m} \exp \left[-\frac{1}{2}\left(\mathbf{y}^{*}-\overline{\mathbf{x}}_{i}\right)^{\mathrm{T}}\left\{\left(D_{1}^{-1}+D_{2}^{-1}\right)^{-1}+\left(h^{2} C\right)\right\}^{-1}\left(\mathbf{y}^{*}-\overline{\mathbf{x}}_{i}\right)\right]
\end{aligned}
$$

Where the term $\mathbf{y}^{*}$ is defined as:

$$
\mathbf{y}^{*}=\left(D_{1}^{-1}+D_{2}^{-1}\right)^{-1}\left(D_{1}^{-1} \overline{\mathbf{y}}_{1}+D_{2}^{-1} \overline{\mathbf{y}}_{2}\right)
$$

The denominator of the LR is given by:

$$
\begin{aligned}
f_{1}\left(\overline{\mathbf{y}}_{1}, \overline{\mathbf{y}}_{2} \mid U, C\right) & =(2 \pi)^{-p}|C|^{-1}\left(m h^{p}\right)^{-2} \prod_{l=1}^{2}\left[\left|D_{l}\right|^{-\frac{1}{2}}\left|D_{l}^{-1}+\left(h^{2} C\right)^{-1}\right|^{-\frac{1}{2}}\right. \\
& \left.\times \sum_{i=1}^{m} \exp \left\{-\frac{1}{2}\left(\mathbf{y}^{*}-\overline{\mathbf{x}}_{i}\right)^{\mathrm{T}}\left(D_{l}+h^{2} C\right)^{-1}\left(\mathbf{y}^{*}-\overline{\mathbf{x}}_{i}\right)\right\}\right]
\end{aligned}
$$

In the equations above, $U$ is the within-source covariance matrix, $C$ is the between-source covariance matrix, $h$ is a smoothing parameter, $m$ are members (e.g., glass fragments) of a population, $\boldsymbol{x}$ are the measurements of samples within the background population, $\boldsymbol{y}$ are 
the measurements of the known (control) and questioned (recovered) samples, and $D$ is the variance-covariance matrix of the known and questioned sample. ${ }^{40}$ Unfortunately, the MVK model can lead to extremely large or small LRs, providing an unreasonable weight of evidence; this is the case for LA-ICP-MS glass data. The extreme LRs are likely a result of the high dimensionality of the data (i.e., many variables). Thus, a post-hoc calibration may be necessary in order to reduce the feature-based LR to more reasonable values. Calibration is accomplished by treating the LR as a score, rather than an actual likelihood ratio, and then transforming the score into a LR.

Vergeer, et al. and van Es, et al. reported one method for calibration that involves the use of density models followed by the empirical lower and upper bound (ELUB) method to limit the LR output. ${ }^{44-45}$ The distribution of the same-source LR scores (using the MVK model) was modeled using a double exponential decay and the distribution of the different-source LR scores was modeled using a KDE. ${ }^{44}$ To compute the calibrated LR for a pairwise comparison, first the LR score is calculated using the MVK model. The numerator of the calibrated LR is given by the probability of the score using the samesource distribution (in this case, a double exponential decay). The denominator of the calibrated LR is given by the probability of the score using the different-source KDE. The upper and lower limit for the calibrated LR is computed using a normalized Bayes error-rate (NBE) plot, which plots the $\log _{10}$ EU ratio against the $\log _{10} \mathrm{LR}_{\mathrm{th}}{ }^{45}$ The expected utility (EU) ratio is the ratio of the EU for the neutral case (in which the LR is always equal to 1) and the EU for the LR system: 


$$
\begin{aligned}
& \frac{E U \text { (neutral) }}{E U(L R \text { system })} \\
& =\frac{P_{H_{p}, 1 \leq L R_{t h}}+L R_{t h} \times P_{H_{d}, 1>L R_{t h}}}{\left(N_{C M L R}+n_{H_{p}, L R \leq L R_{t h}}\right) /\left(N_{C M L R}+n_{H_{p}}\right)+L R_{t h} \times\left(N_{C M L R}+n_{H_{d}, L R>L R_{t h}}\right) /\left(N_{C M L R}+n_{H_{d}}\right)}
\end{aligned}
$$

In the equation above, $H_{p}$ is the prosecutor's hypothesis (an association, sometimes given as $\mathrm{H}_{1}$ ), $H_{d}$ is the defense's hypothesis (an exclusion, sometimes given as $\mathrm{H}_{2}$ ), $n_{H p, L R \leq L R t h}$ is the number of calibrated same-source LRs in which the LR $\leq \mathrm{LR}_{\mathrm{th}}, n_{H d, L R>L R t h}$ is the number of calibrated different-source LRs in which the $\mathrm{LR}>\mathrm{LR}_{\mathrm{th}}$, and $N_{C M L R}$ is the number of consequential misleading LRs. The EU ratio is calculated for a wide range of threshold LRs, $\mathrm{LR}_{\mathrm{th}}$, in order to generate the NBE plot. The upper and lower limits for the calibrated LR are the points where the NBE plot crosses the $\mathrm{x}$-axis $(\mathrm{y}=0)$. Another method for LR calibration employs the Pool Adjacent Violators (PAV) algorithm, which uses strictly proper scoring rules (SPSRs). ${ }^{42,139-141}$ The ELUB method described above includes one step for calibration and a subsequent step to limit the LR. The PAV transformation, on the other hand, simultaneously calibrates and sets an upper and lower limit to the LR, while still maintaining the discriminating power of the LRs. ${ }^{140}$, ${ }^{142}$ The algorithm gives a non-decreasing transformation for each posterior probability (corresponding to each un-calibrated LR) such that the resulting posterior probabilities are better calibrated. Recall that Bayes theorem is defined as: posterior odds $=$ likelihood ratio $\times$ prior odds .

$$
\frac{P\left(H_{1} \mid E\right)}{P\left(H_{2} \mid E\right)}=\frac{P\left(E \mid H_{1}\right)}{P\left(E \mid H_{2}\right)} \times \frac{P\left(H_{1}\right)}{P\left(H_{2}\right)}
$$


The aim of the PAV algorithm is to minimize the empirical cross entropy (ECE) of the set of transformed posterior probabilities. ${ }^{42}$ The ECE is a measure of accuracy for all possible prior probabilities (the smaller the ECE, the better the accuracy) and can be defined as follows:

$$
\begin{gathered}
E C E=\frac{P\left(H_{1} \mid I\right)}{N_{1}} \times \sum_{i: H_{1} \text { is true }} \log _{2}\left(1+\frac{1}{L R_{i} \times O\left(H_{1} \mid I\right)}\right) \\
+\frac{P\left(H_{2} \mid I\right)}{N_{2}} \times \sum_{j: H_{2} \text { is true }} \log _{2}\left(1+L R_{j} \times O\left(H_{1} \mid I\right)\right)
\end{gathered}
$$

In the equation above, $N_{1}$ and $N_{2}$ are the number of cases for which $H_{1}$ and $H_{2}$ are true, respectively. $O\left(H_{l} \mid I\right)=P\left(H_{1} \mid I\right) \div P\left(H_{2} \mid I\right)$ are the prior odds and $I$ is the information (excluding the evidence, $E$ ) in the case. In Zadora et al., a detailed description of ECE and the PAV algorithm are given. ${ }^{42}$ 


\section{ELEMENTAL ANALYSIS OF PRINTING INKS}

This chapter presents the discrimination and association potential of Scanning Electron Microscopy-Energy Dispersive Spectroscopy (SEM-EDS) for four types of printing inks: inkjet, toner, offset, and intaglio. The SEM-EDS results are also compared to the more sensitive technique Laser Ablation-Inductively Coupled Plasma-Mass Spectrometry (LAICP-MS). Additionally, a smaller subset of toner and inkjet samples were analyzed using Laser Induced Breakdown Spectroscopy (LIBS). Finally, a searchable ink database (the Forensic Ink Analysis and Comparison System, FIACS) was developed with collaborators CoVar and Applied Spectra.

\subsection{Sample Collection and Preparation}

A total of 319 ink specimens were collected for this study: 78 inkjet inks, 76 toner inks, 79 offset inks, and 86 intaglio inks. A description of each collection as well as the preparation procedure for each ink type is discussed below. A complete list of all inks in the collection can be found in Appendix A.

\subsubsection{Inkjet Inks}

The 78 inkjet samples were collected from 9 different manufacturers and included the four colors used for inkjet printing: black (K), cyan (C), magenta (M), and yellow (Y). The inkjet cartridges were purchased from OEM sources in order to identify the manufacturing source. Figure 9 shows the distribution by brand for the inkjet collection 
set. As can be seen, the majority $(\sim 31 \%)$ of the inkjet specimens were sampled from cartridges by Hewlett Packard, the world's largest ink provider.

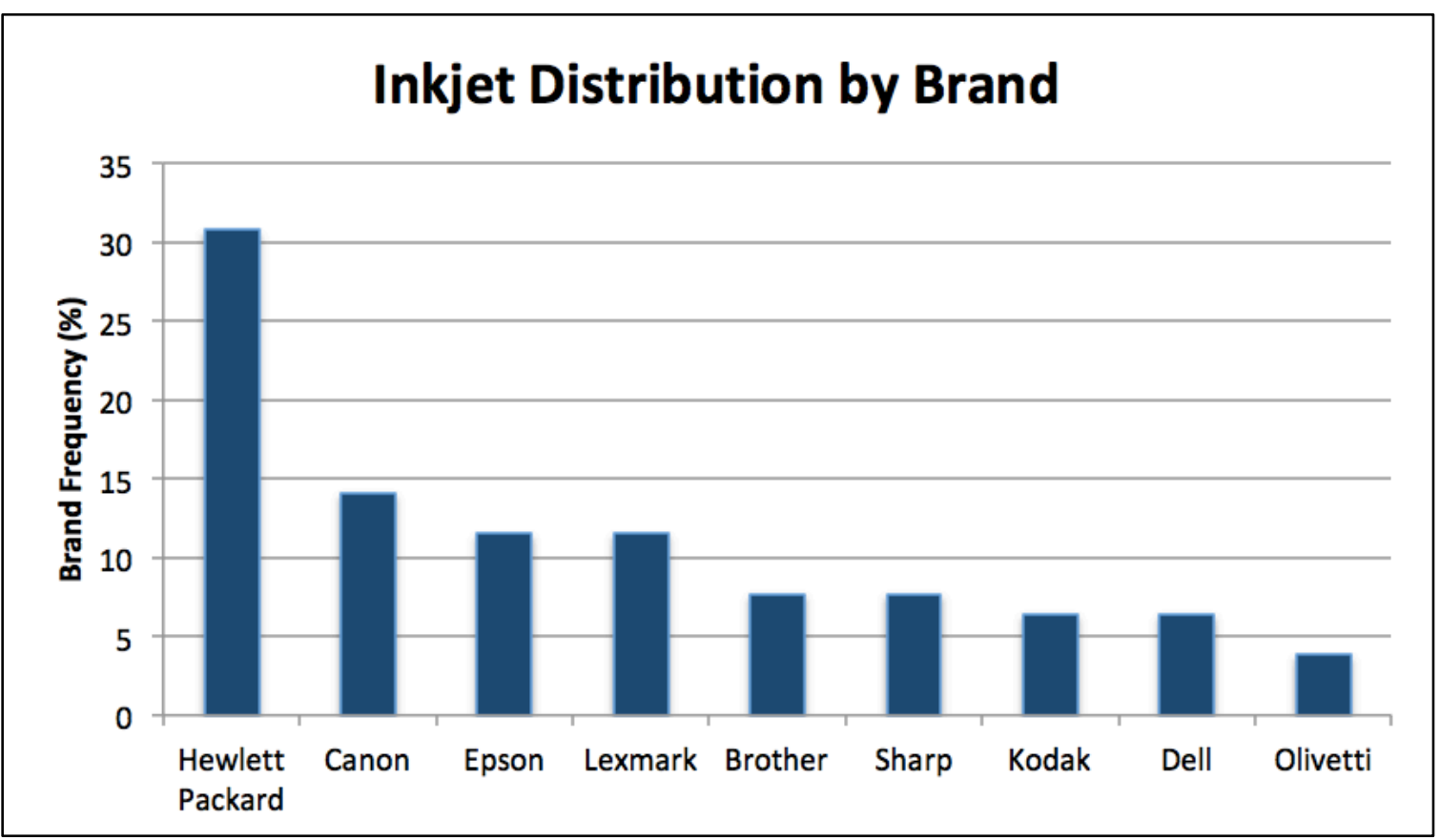

Figure 9 - Distribution of inkjet collection by manufacturing brand

Figure 10 shows the distribution of the inkjet collection by color. Black ink is the most commonly encountered color in forensic casework. Therefore, a greater number of black inkjet cartridges were sampled $(\sim 42 \%)$, compared to the remaining three colors $(16-$ $21 \%$ ). Some cartridges were labeled as "light cyan" or "light magenta;" these are included within the main color category in Figure 10. In some cases, the three ink colors (C, M, and Y) were housed in individual cartridges, while in other cases, the three colors were all housed in a single cartridge. The latter are referred to as "tri-color" cartridges in Appendix A. 


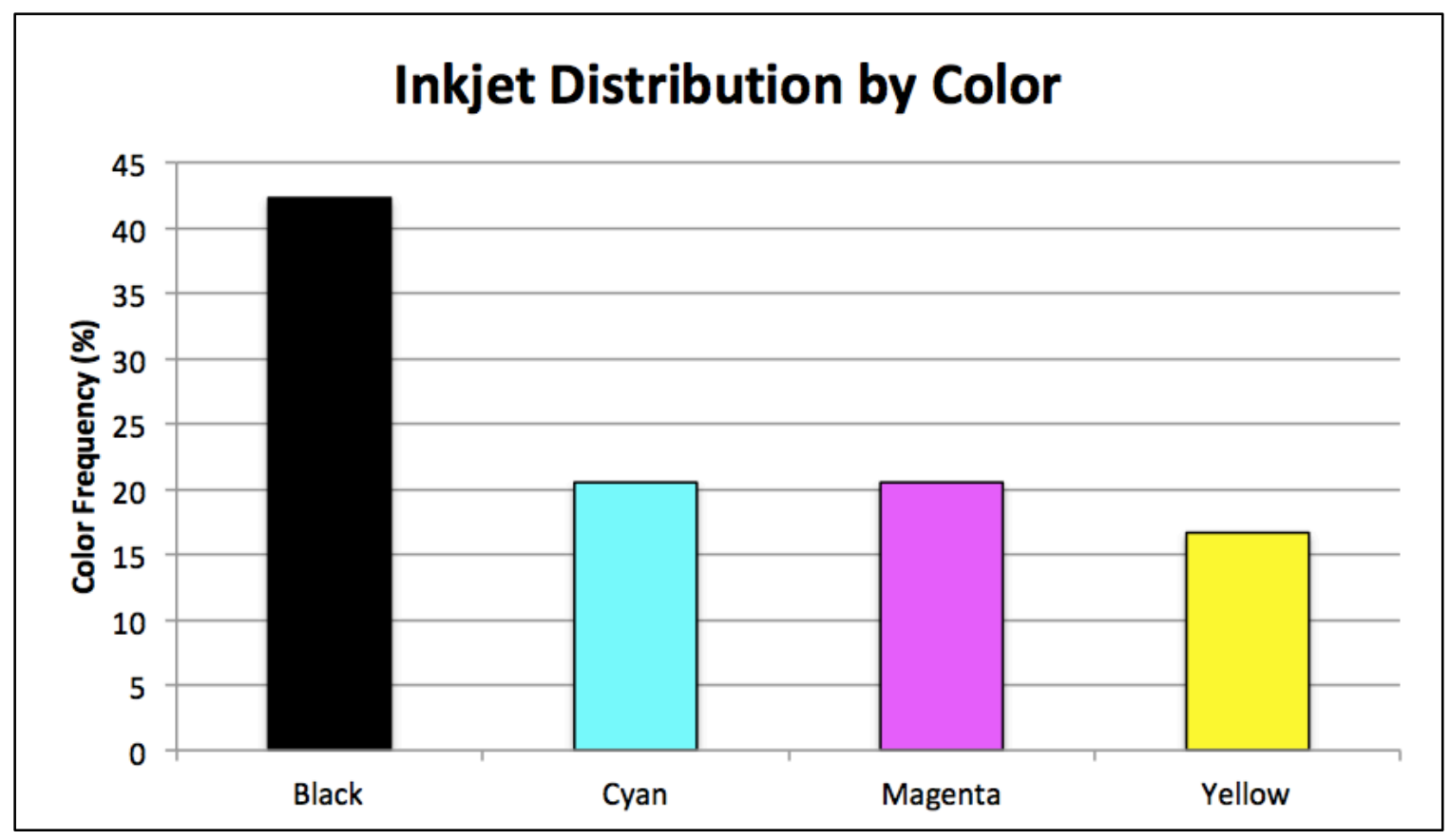

Figure 10 - Distribution of inkjet collection by ink color

In cases where the printer for a corresponding ink cartridge was available, the inkjet specimens were prepared by printing text and/or filled squares directly onto an 8.5 " by 11" piece of Whatman 42 filter paper. Whatman 42 paper was selected as the substrate since it has trace concentrations of elements of interest and was thus unlikely to interfere with the ink's elemental profile. All printouts were collected from pure color cartridges (CMYK); this was verified by inspecting the printouts under a microscope to ensure that there were no color mixtures. If the necessary printer was unavailable, the ink specimens were prepared by extracting the ink from the cartridge and depositing the ink onto small ( 1" by 1.5") pieces of Whatman 42 paper. Some cartridges contained sponges that were saturated with the ink, while other cartridges housed the liquid ink in a small inner compartment. In the former case, the sponge was removed and used to smear the ink onto 
the Whatman 42 paper. In the latter case, $5 \mu 1$ of the ink was pipetted onto the Whatman 42 paper using a micropipette. Figure 11 shows examples of inkjet specimens prepared using different methods.

The inkjet ink deposited homogeneously and was easily absorbed into the Whatman 42 paper fibers. The printouts typically penetrated the paper up to $60-95 \mu \mathrm{m}$. The inks that were manually deposited nearly penetrated through the whole thickness of the paper substrate $(200-250 \mu \mathrm{m})$.

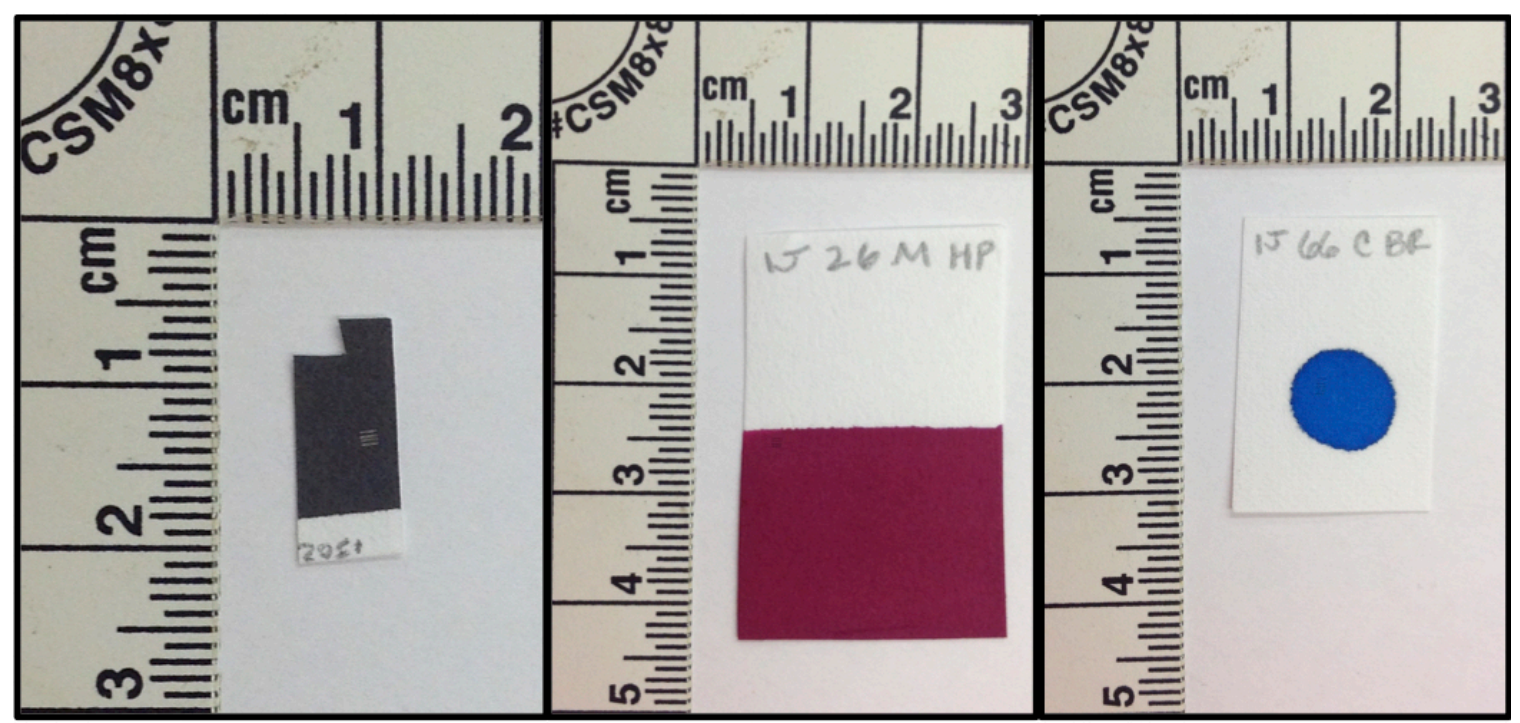

Figure 11 - Example of inkjet specimens: printed (left), smeared with cartridge sponge (middle), and pipetted with micropipette (right)

\subsubsection{Toner Inks}

The 76 toner samples originated from 16 different manufacturers and, like inkjets, included the four colors used for color laser printing: black, cyan, magenta, and yellow. Most cartridges were purchased from OEM sources in order to identify the manufacturing source. However, twenty toner samples have unknown model, manufacturer, and lot number (see Appendix A); these are chemically prepared toners that were provided by 
the U.S. Secret Service. Figure 12 shows the distribution by brand for the toner collection set. Once again, the majority $(\sim 33 \%)$ of the toner specimens were sampled from cartridges by Hewlett Packard. The remaining 15 manufacturers each comprised 1-8\% of the collection.

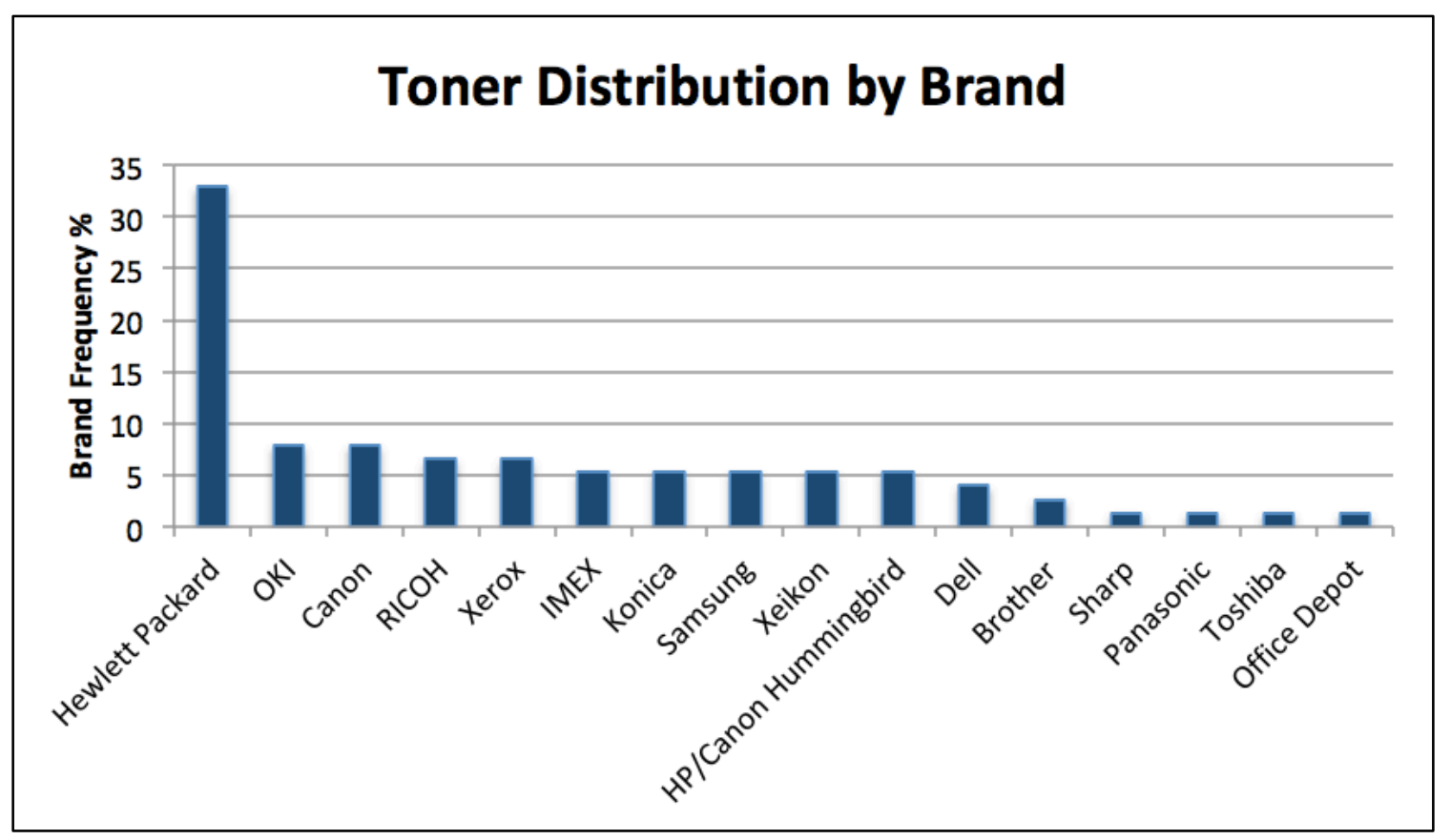

Figure 12 - Distribution of toner collection by manufacturing brand

Figure 13 shows the distribution of the toner collection by color. Approximately half of the specimens were black ink, the most commonly encountered color in forensic casework. The remaining three colors each represented $15-17 \%$ of the collection. Unlike inkjet inks, all toner inks were housed in individual cartridges; that is, there were no "tricolor" cartridges. 


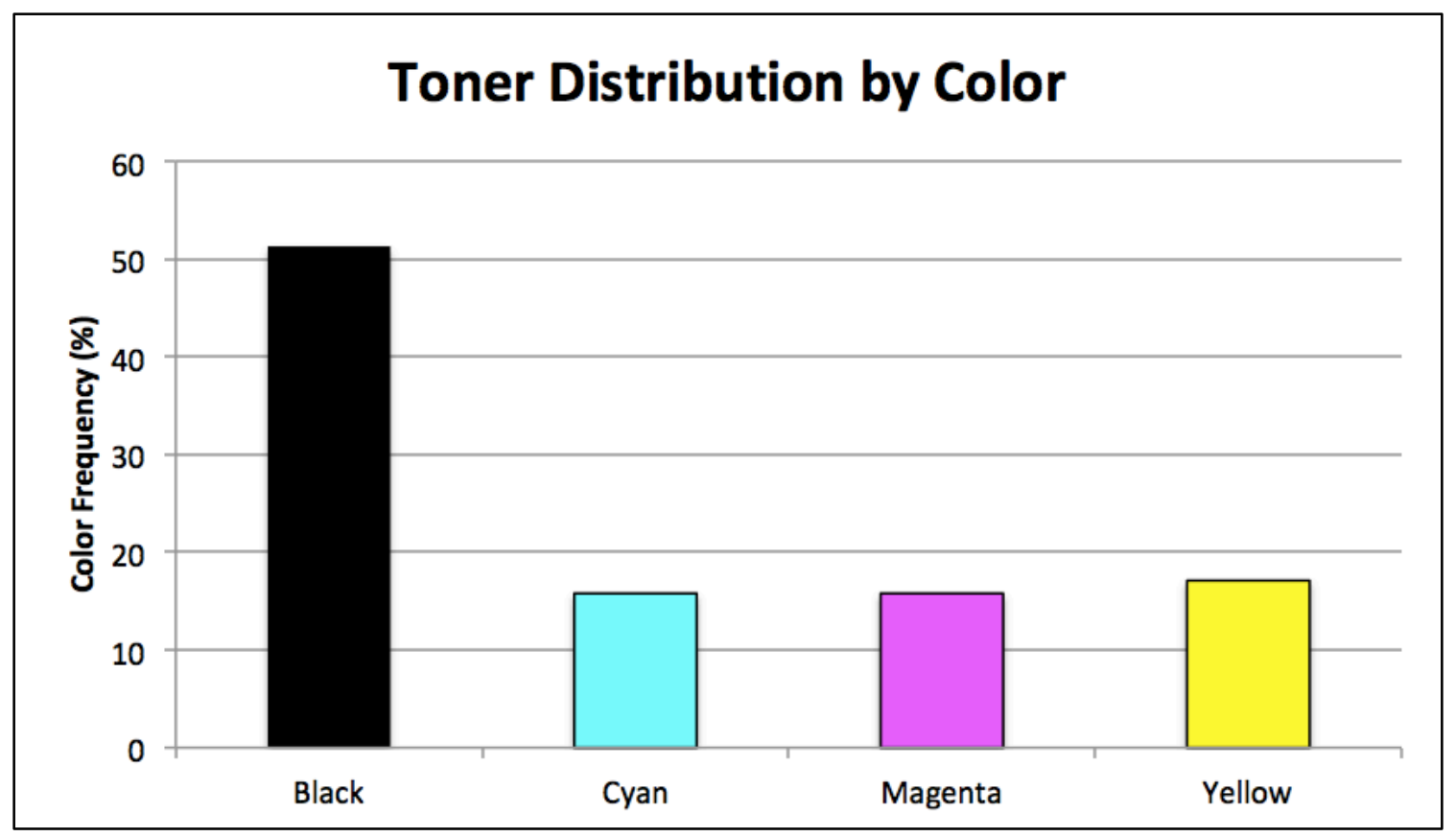

Figure 13 - Distribution of toner collection by ink color

As was the case for inkjets, if the printer for a corresponding ink cartridge was available, the toner specimens were prepared by printing text and/or filled squares directly onto an 8.5 " by 11 " piece of Whatman 42 filter paper. Only printers for some of the black ink cartridges were available. For the remaining black ink and all CMY inks, the powdered ink was removed from the cartridge with a plastic disposable pipet that was previously cut into the shape of a spatula. Approximately 0.1 grams of the powdered ink was placed onto a small ( 1' by 1.5 ") piece of Whatman paper that was placed atop a glass microscope slide. The glass slide (with the paper and ink on top) was then placed on the surface of a hot block set at a temperature between 140 to $180{ }^{\circ} \mathrm{C}$. The powdered ink was allowed to melt and a glass cover slide was used to smear the melted ink on the Whatman paper. Three toner inks were a liquid rather than powder; these were prepared by heating 
the ink on a glass microscope slide until the ink was viscous enough to smear onto the paper substrate using a glass cover slide. Figure 14 shows examples of toner specimens.

Toner ink sits heterogeneously on top of the paper substrate rather than penetrating through the paper fibers. The printouts typically yielded an ink layer of about 50-60 $\mu \mathrm{m}$, while the manual deposition method described above typically resulted in an ink layer of about $70-90 \mu \mathrm{m}$.

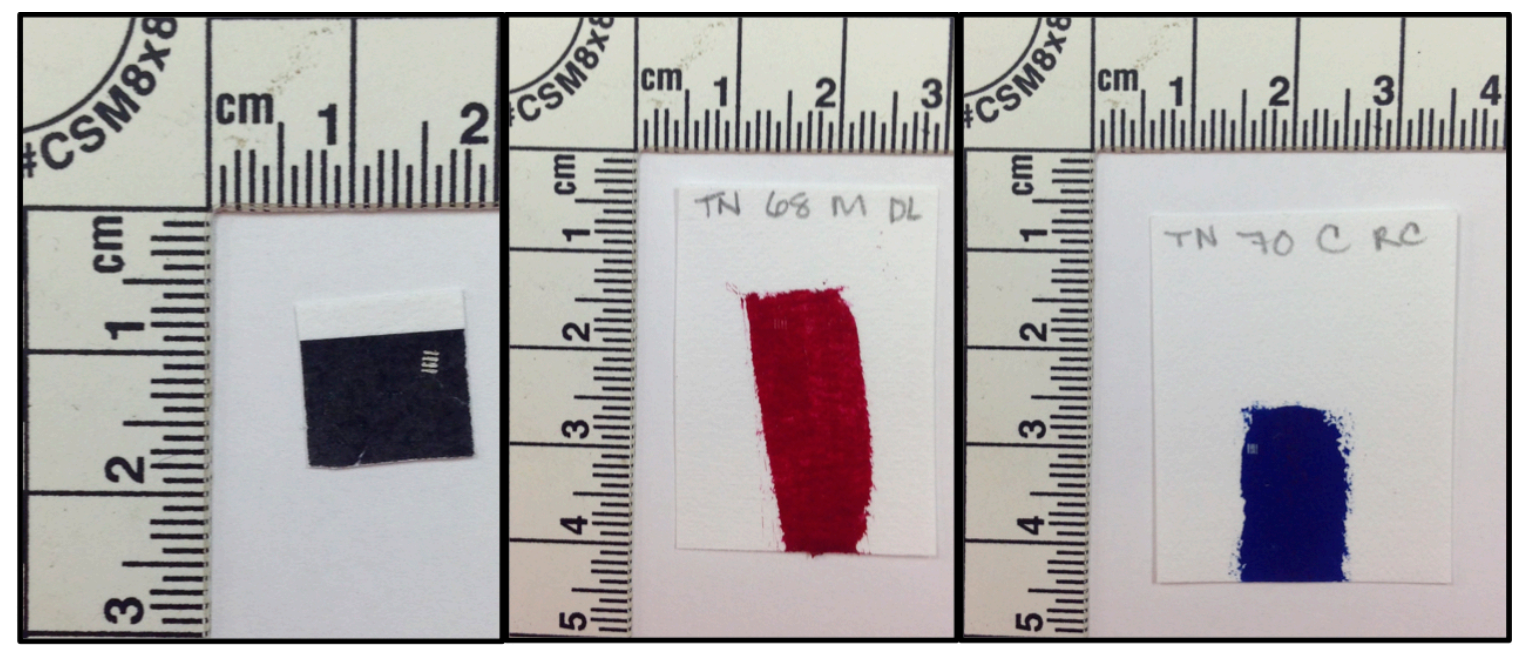

Figure 14 - Examples of toner specimens: printed (left), heated and smeared powder (middle), and heated and smeared liquid (right)

\subsubsection{Offset Inks}

The 79 offset samples included a variety of sample types: printouts from 5 different manufacturers, the raw paste from 2 manufacturers, and several documents (passport, visa, and business card). The majority of the samples were provided by the Homeland Security Investigations (HSI) Forensic Laboratory. Figure 15 shows the distribution of offsets by sample type, most of which were printouts $(\sim 50 \%)$. Offset inks are available in a variety of colors, not only the four colors typically used for color inkjet or laser 
printing (CMYK); approximately 21 colors were included in the offset collection. Most of the offset specimens were printed either on conventional or specialty paper, not Whatman 42 paper. Thus, an important consideration for the offset collection was the potential elemental contribution from the paper substrate.

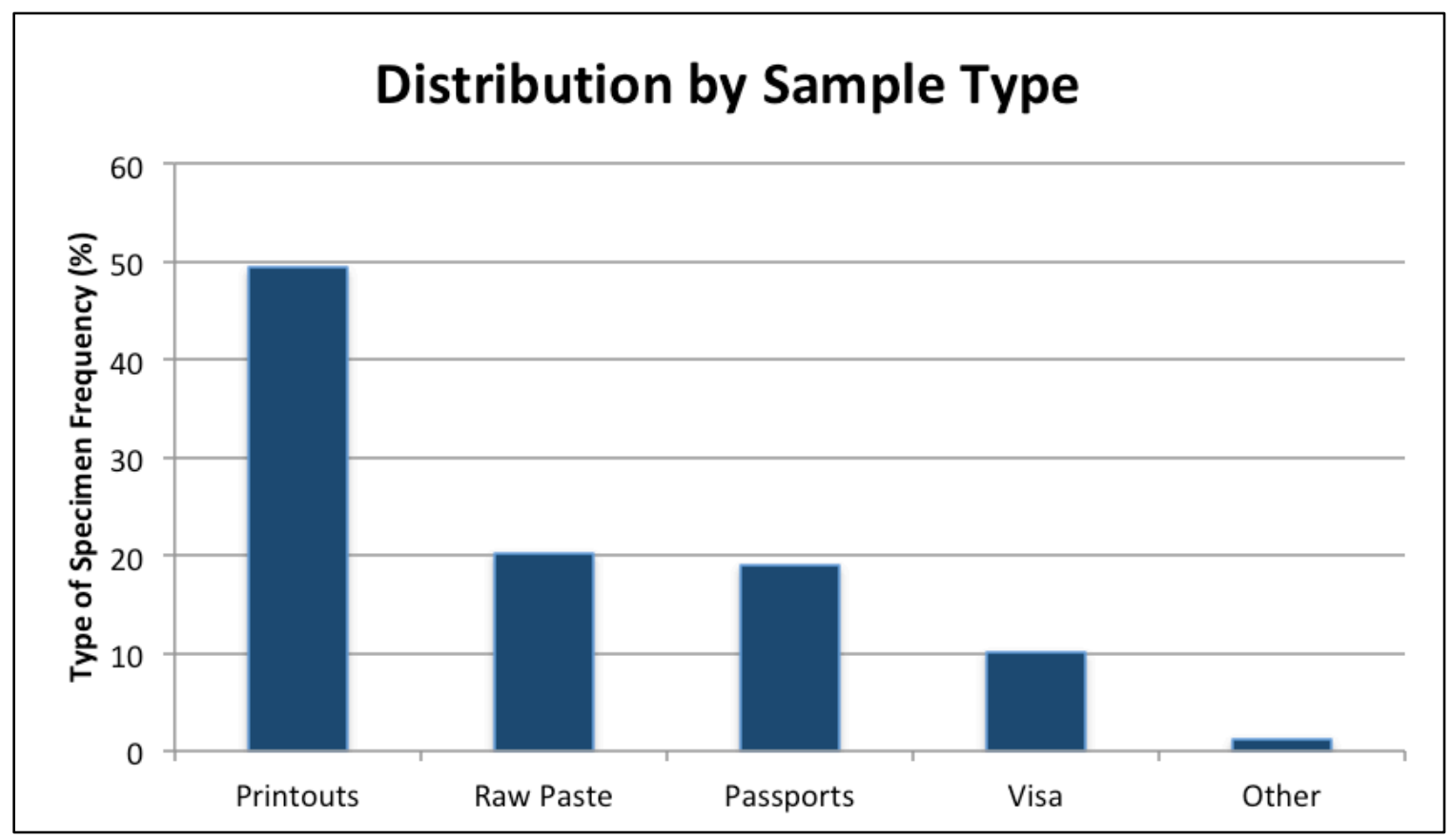

Figure 15 - Distribution of offset collection by sample type

Since most of the offset samples were printouts provided by HIS, sample preparation simply consisted of cutting the samples into smaller sections using a scalpel. The offset samples that were received as a raw paste were prepared using a similar procedure as the one described for toners. The raw paste was first homogenized using a vortex. A small amount $(<0.05 \mathrm{ml})$ of the paste was then added to a small $\left(\sim 1\right.$ ' by $\left.1.5^{\prime \prime}\right)$ piece of Whatman 42 paper, which was placed on top of a glass microscope slide. A glass cover slide was then used to smear the ink on the paper substrate; since the ink was a paste, 
rather than a powder, no heating was necessary in order to smear the ink. The smeared paste was then allowed to dry at room temperature in a fume hood. Figure 8 shows examples of offset specimens.

Like inkjets, the offset inks partially penetrated the paper fibers. However, the ink film was typically thinner $(\sim 4-23 \mu \mathrm{m})$. In some cases, the ink film was so thin that the carbonate fillers present in the paper substrate are still visible.

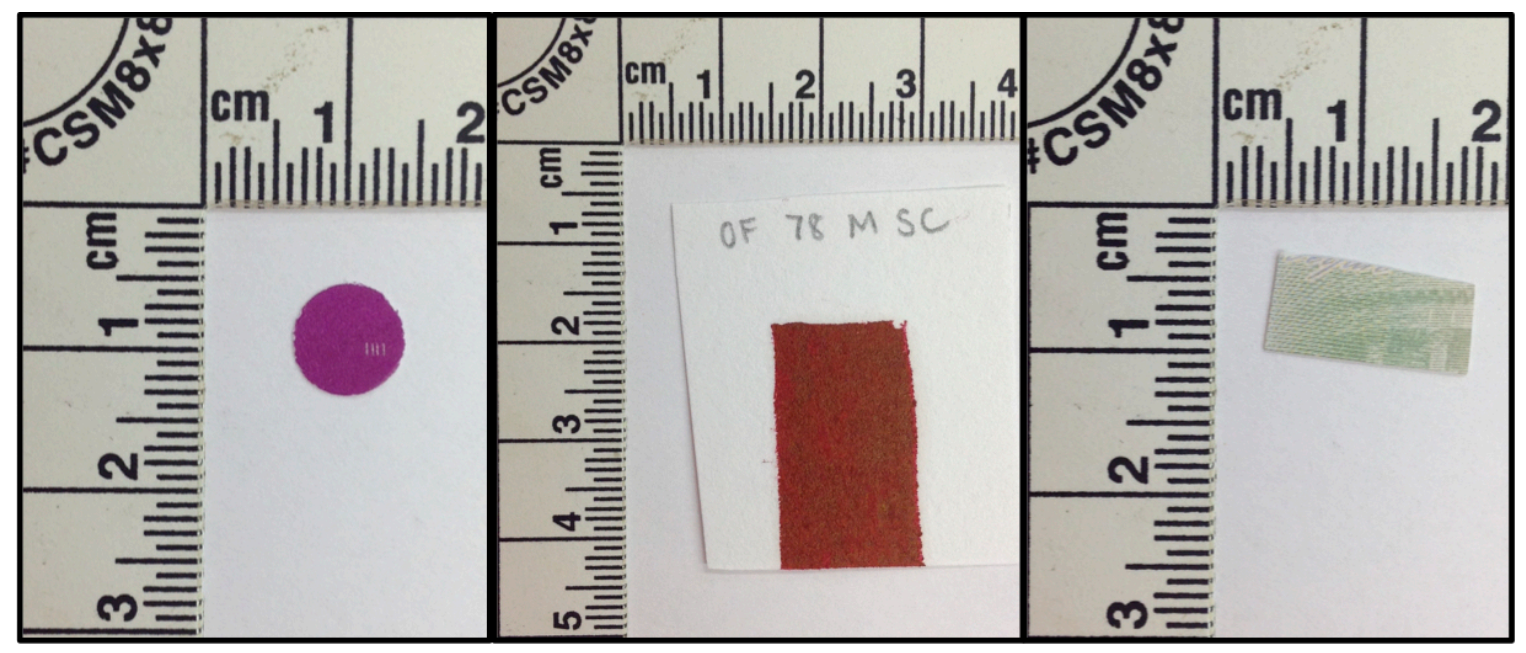

Figure 16 - Examples of offset specimens: printed (left), smeared raw paste (middle), and cutout from visa (right)

\subsubsection{Intaglio Inks}

The majority of the 86 intaglio samples originated from banknotes of 24 different countries. Keesing Documentchecker was used to verify the areas of the banknotes that were printed with intaglio ink. In many cases, a single banknote had several different colors of intaglio ink in different areas. Thus, some intaglio specimens originated from the same banknote. Some banknotes (from New Zealand) were composed of a flexible polymer substrate, rather than paper. Figure 17 shows the distribution of intaglio samples 
by country of origin. The greatest number of samples originated from Costa Rica, followed by Russia, Europe, and the United States. Apart from the banknotes, a few samples originated from a visa or from business cards. Like offset inks, intaglio inks include a variety of colors (in this case, 17 colors). For the intaglio set, the ink was printed on a variety of substrates, thus the elemental contribution from the paper or polymer was an important consideration. However, since intaglio ink is raised on the surface of the paper, the paper contribution was expected to be less problematic than for offset inks.

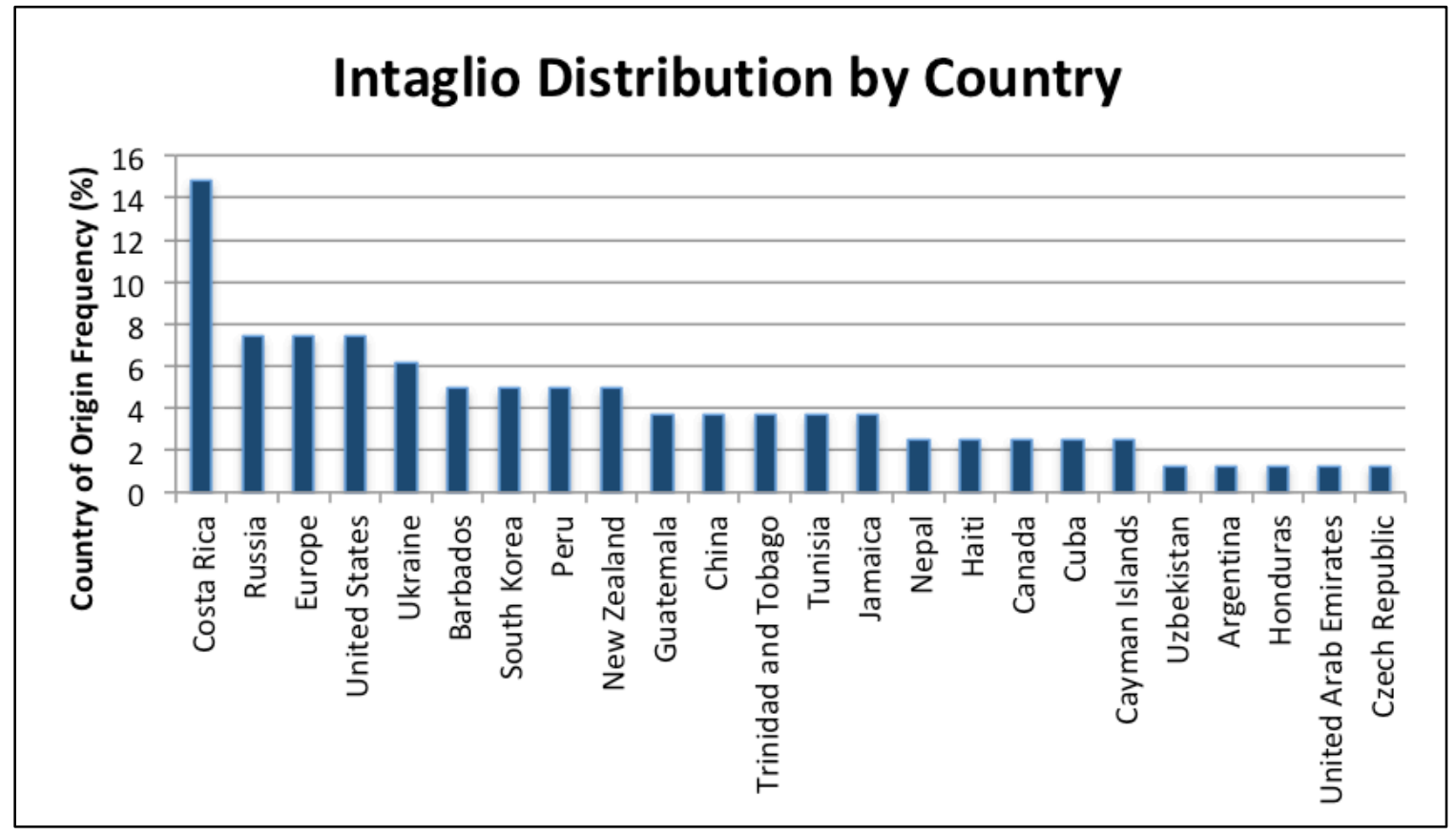

Figure 17 - Distribution of intaglio collection by country of origin

Since all intaglio specimens were printed, sample preparation simply consisted of cutting the samples into small pieces using a scalpel. Figure 18 shows an example of a banknote (from Barbados) along with the intaglio areas that were sampled circled in red. 
Like toners, intaglio ink is deposited heterogeneously on top of the paper, or polymer, substrate. The thickness of the ink film was in the range of $19-45 \mu \mathrm{m}$ for this collection set.

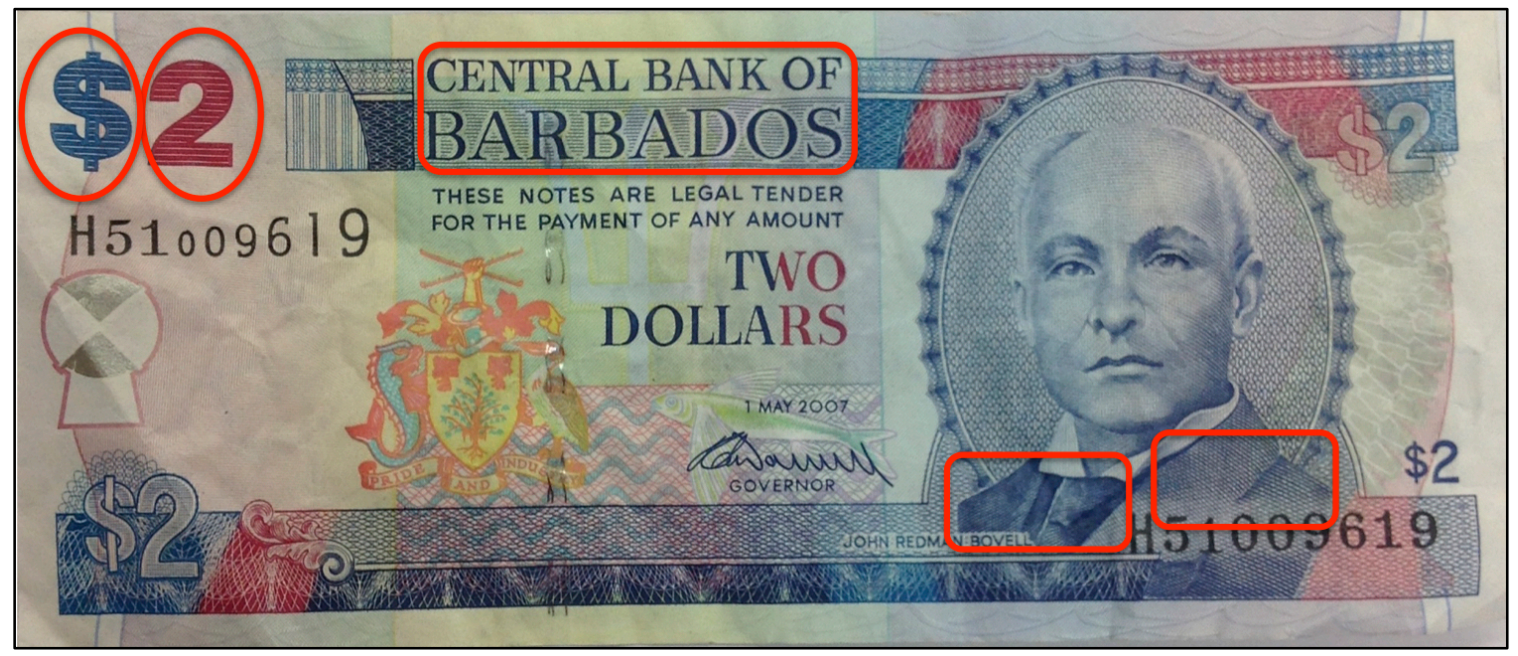

Figure 18 - Barbados banknote with the intaglio areas that were sampled circled in red

Table 10 - Summary of the main characteristics of the four ink types: inkjet, toner, offset, and intaglio

\begin{tabular}{|c|c|c|c|c|}
\hline & INKJET & TONER & OFFSET & INTAGLIO \\
\hline $\begin{array}{l}\text { Number of } \\
\text { Samples }\end{array}$ & 78 & 76 & 79 & 86 \\
\hline Film Thickness & $60-95 \mu \mathrm{m}$ & $20-95 \mu \mathrm{m}$ & $4-23 \mu \mathrm{m}$ & $19-45 \mu \mathrm{m}$ \\
\hline Distribution & Homogeneous & Heterogeneous & Homogeneous & Heterogeneous \\
\hline Deposition & $\begin{array}{l}\text { Embedded into } \\
\text { paper fibers }\end{array}$ & $\begin{array}{l}\text { Deposited on } \\
\text { paper }\end{array}$ & $\begin{array}{l}\text { Thin film, } \\
\text { partially } \\
\text { embedded }\end{array}$ & $\begin{array}{c}\text { Deposited on } \\
\text { paper, raised ink }\end{array}$ \\
\hline $\begin{array}{c}\text { Paper } \\
\text { Contribution }\end{array}$ & $\begin{array}{c}\text { Paper } \\
\text { contribution }\end{array}$ & $\begin{array}{l}\text { Limited paper } \\
\text { contribution }\end{array}$ & $\begin{array}{c}\text { Paper } \\
\text { contribution }\end{array}$ & $\begin{array}{l}\text { Limited paper } \\
\text { contribution }\end{array}$ \\
\hline
\end{tabular}

Table 10 summarizes the main differences between the four ink types: inkjet, toner, offset, and intaglio. Inkjet and offset are homogeneously distributed, are embedded into the paper fibers, and are expected to have paper contribution. However, in the case of inkjet, since Whatman 42 paper was used as the substrate, the paper contribution is 
minimized. Toner and intaglio are heterogeneously distributed, are deposited on top of the paper, and are expected to have limited paper contribution.

\subsection{SEM-EDS Analysis of Printing Inks}

The elemental analysis and imaging was conducted on a Philips XL 30 Scanning Electron Microscope equipped with an EDX detector (Philips, The Netherlands and EDAX, USA, respectively). The optimized parameters for elemental analysis of printing inks as well as the elements detected (signal-to-noise ratio $\geq 3$ ) in each ink type are listed Table 11. The working distance was adjusted within the specified range to ensure that similar counts were obtained for each quality control. The only differences in the optimized parameters between the different ink types were the sampling area and the time constant. For toners and inkjets, the analysis was conducted over the entire area at a magnification of 500x $(\sim 400 \times 500 \mu \mathrm{m})$. However, since the ink lines for some intaglio and offset samples were too narrow, the sampling area had to be reduced for all intaglio and offset specimens ( $\sim 40$ $\mathrm{x} 160 \mu \mathrm{m}$ ) in order to avoid sampling the paper flanking the ink line. The time constant for each ink type was selected to obtain a dead time below 40\%. SEM imaging was conducted under low vacuum (1.5 mBar), with an accelerating voltage of $30 \mathrm{kV}$, a magnification of 500x, and a working distance in the range of 13.0-14.0 mm for inkjet, offset, and intaglio samples. Imaging of toners was found to be more important since differences in particle shape and size may aid in the differentiation of the toners. Therefore, the parameters for the SEM imaging of the toners were adjusted as follows to improve the resolution of the images: low vacuum, accelerating voltage of $30 \mathrm{kV}$, magnification of 1250x, and working distance of $9 \mathrm{~mm}$. All ink samples were prepared 
by cutting a small piece of the ink and paper, typically less than $0.5 \times 0.5 \mathrm{~cm}$, and pasting it on an aluminum SEM stub using a carbon conductive tab (see Figure 20).

Table 11 - Optimized SEM-EDS parameters for the analysis of printing inks; elements detected $(\mathrm{SNR} \geq 3)$ in the ink and paper are also reported

\begin{tabular}{|c|c|c|c|c|}
\hline & INKJET & TONER & OFFSET & INTAGLIO \\
\hline $\begin{array}{c}\text { Energy } \\
(\mathrm{keV})\end{array}$ & 30 & 30 & 30 & 30 \\
\hline Magnification & 500 & 500 & 500 & 500 \\
\hline $\begin{array}{l}\text { Sampling Area } \\
(\mu \mathrm{m})\end{array}$ & $400 \times 500$ & $400 \times 500$ & $400 \times 500$ & $400 \times 500$ \\
\hline Time Constant & 2.0 & 2.0 & 8.0 & 8.0 \\
\hline $\begin{array}{c}\text { Working } \\
\text { Distance }(\mu \mathrm{m})\end{array}$ & $13.0-14.0$ & $13.0-14.0$ & $13.0-14.0$ & $13.0-14.0$ \\
\hline $\begin{array}{l}\text { Sampling } \\
\text { Time (Ls) }\end{array}$ & 1000 & 1000 & 1000 & 1000 \\
\hline $\begin{array}{c}\text { Elements } \\
\text { Detected in } \\
\text { Ink }\end{array}$ & $\begin{array}{c}\mathrm{Cu}, \mathrm{K}, \mathrm{Mg}, \mathrm{Na}, \\
\mathrm{S}\end{array}$ & $\begin{array}{c}\mathrm{Al}, \mathrm{Ba}, \mathrm{Ca}, \mathrm{Cl} \\
\mathrm{Cr}, \mathrm{Cu}, \mathrm{Fe}, \mathrm{S}, \\
\mathrm{Si}, \mathrm{Sr}, \mathrm{Ti}, \mathrm{W}, \\
\text { Zn, Zr }\end{array}$ & $\begin{array}{c}\mathrm{Al}, \mathrm{Ba}, \mathrm{Cl}, \mathrm{Cu} \text {, } \\
\mathrm{K}, \mathrm{Mn}, \mathrm{Mo}, \mathrm{S} \text {, } \\
\mathrm{Si}, \mathrm{Sr}, \mathrm{Ti}, \mathrm{W}, \\
\mathrm{Zn}, \mathrm{Fe}, \mathrm{Mg}\end{array}$ & $\begin{array}{c}\mathrm{Al}, \mathrm{Ba}, \mathrm{Br}, \mathrm{Ca} \text {, } \\
\mathrm{Cl}, \mathrm{Co}, \mathrm{Cr}, \mathrm{Cu} \text {, } \\
\text { F, Fe, K, Mg, P, } \\
\text { S, Si, Ti, Zn }\end{array}$ \\
\hline $\begin{array}{l}\text { Elements } \\
\text { Detected in } \\
\text { Paper }\end{array}$ & None & None & $\begin{array}{l}\mathrm{Mg}, \mathrm{Al}, \mathrm{Si}, \mathrm{S} \\
\mathrm{Cl}, \mathrm{Ca}, \mathrm{Ti}, \mathrm{Zn}\end{array}$ & $\begin{array}{c}\mathrm{Na}, \mathrm{Mg}, \mathrm{Al}, \mathrm{Si}, \\
\mathrm{P}, \mathrm{S}, \mathrm{Cl}, \mathrm{K}, \mathrm{Ca}, \\
\quad \mathrm{Ti}, \mathrm{Fe}, \mathrm{Cu}\end{array}$ \\
\hline
\end{tabular}

To assess the discrimination potential of SEM-EDS for the analysis of printing inks, pairwise comparisons were conducted using spectral overlay. Prior to spectral overlay comparisons, the EDS spectra were processed using the EDX2008 software (Version 1.2) and normalized to the sum of all peaks; each spectrum was also inspected manually to account for sum peaks, escape peaks, and regions of overlap (e.g., Ti K series and Ba L series). The criteria for deciding whether two samples could be differentiated are described in our previous publication. ${ }^{29}$ Briefly, if the range in intensity for the replicates of one sample did not overlap with the range in intensity for the other sample for at least 
one element, that pair was said to be distinguishable. Only those elements that were present at a signal-to-noise ratio (SNR) of at least 3:1 were used for discrimination. In order to avoid bias during the spectral overlay comparisons, two different analysts conducted the data examination separately; additionally, a set of duplicate samples was provided as blind tests. The total number of comparison pairs is given by the following formula, where $n$ is the total number of samples.

$$
n \times \frac{(n-1)}{2}
$$

The discrimination (reported as $\%$ ) provides a measure of how many sample pairs were distinguishable out of the total number of comparison pairs. The false inclusion (also reported as $\%$ ) is the number of ink samples that were not discriminated, even though these originated from different sources and is equal to one hundred minus the percent discrimination. The correct association rate is reported as the number of known duplicate controls that were correctly found to be associated with the respective duplicate sample. Finally, the false exclusion rate provides the number of duplicate controls that were incorrectly discriminated from the respective duplicate sample; thus, the percent false exclusion is calculated by subtracting the percent correct association from one hundred. Since each of the four ink types are easily distinguishable from one another either visually and/or microscopically, the discrimination, association, and false inclusion and exclusion rates are reported for samples within each ink type rather than for the collective sample set. 


\subsubsection{Inkjet Inks}

SEM-EDS discriminated $78.4 \%$ (2355 out of 3003 comparison pairs) of the 78 inkjet samples analyzed. Only five elements were detectable in inkjets: $\mathrm{Cu}, \mathrm{K}, \mathrm{Mg}, \mathrm{Na}$, and $\mathrm{S}$. Of the 648 pairs that could not be discriminated, 299 pairs were neither same-brand nor same-color comparisons, indicating the technique's limited utility for the analysis of inkjet inks. The low discrimination and high rate of false inclusions $(21.6 \%)$ for the analysis of inkjets by SEM-EDS is not surprising considering the technique's relatively low sensitivity (LOD $1000 \mathrm{ppm}$ ) as well as the low concentration of inorganic content in inkjets. ${ }^{121,143}$ A total of six duplicate samples were analyzed to evaluate the correct association capabilities of SEM-EDS; all six duplicates were correctly associated. However, it should be noted that a greater number of duplicates is required to truly estimate the correct association. Unfortunately, the lengthy analysis time (20 minutes per replicate) made it impractical to analyze a larger set of duplicate samples. Figure 19 shows an example of two discriminated samples (Inkjet 21 and 24) as well as Inkjet 77 compared to its duplicate.

Since the inkjet samples were sampled from pure-color ink cartridges and the four colors are easily distinguishable by visual inspection, the discrimination between same-color pairs was also calculated. Table 12 shows discrimination, correct association, and false inclusions/exclusions for each individual color as well as the entire inkjet collection. Compared to the overall discrimination, the discrimination of the cyan, magenta, and yellow inkjets improved ( $>83 \%$ ). However, the discrimination of the black inkjets decreased to a low $49.2 \%$. Thus, SEM-EDS may not be a suitable technique for the discrimination of black inkjets. 


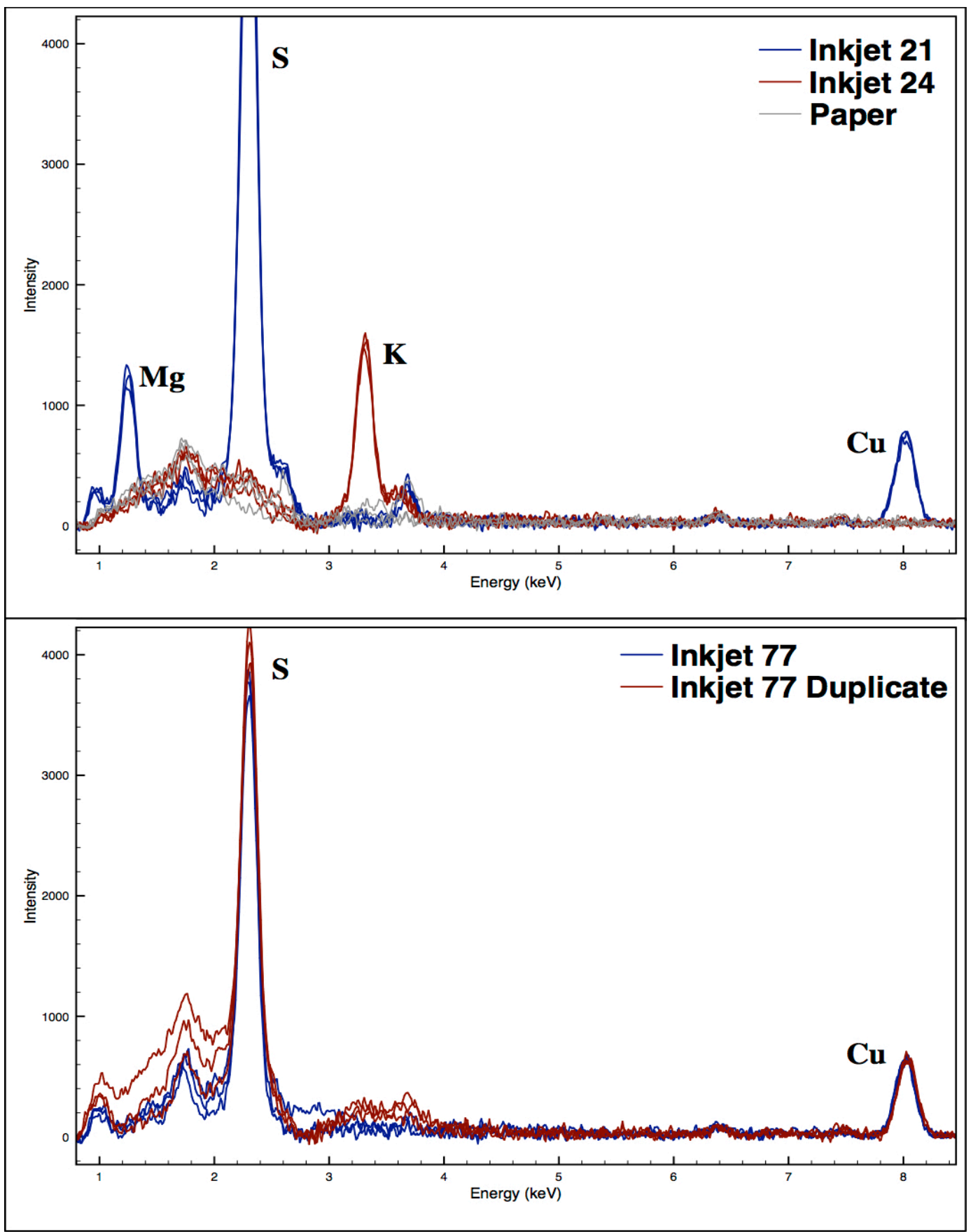

Figure 19 - Example of two discriminated inkjet samples and Whatman 42 paper (top) and example of one sample compared to its duplicate (bottom) 
Table 12 - Discrimination (bolded), correct association (bolded), and false inclusion/exclusion rate for all SEM-EDS inkjet comparisons as well as for each individual color. Values in parentheses indicate either the number of pairwise comparisons or the fraction of discriminated/associated pairs.

\begin{tabular}{cccccc}
\hline & OVERALL & BLACK & CYAN & MAGENTA & YELLOW \\
\hline Number of & 78 & 33 & 16 & 16 & 13 \\
Samples & $(3003)$ & $(528)$ & $(120)$ & $(120)$ & $(78)$ \\
Number of & 6 & 2 & 2 & 1 & 1 \\
Duplicates & $(6)$ & $(2)$ & $(2)$ & $(1)$ & $(1)$ \\
Discrimination & $\mathbf{7 8 . 4 \%}$ & $\mathbf{4 9 . 2 \%}$ & $\mathbf{8 9 . 2 \%}$ & $\mathbf{9 0 . 8 \%}$ & $\mathbf{8 3 . 3 \%}$ \\
Correct & $(2355 / 3003)$ & $(260 / 528)$ & $(107 / 120)$ & $(109 / 120)$ & $(65 / 78)$ \\
Association & $\mathbf{1 0 0 \%}$ & $\mathbf{1 0 0 \%}$ & $\mathbf{1 0 0 \%}$ & $\mathbf{1 0 0 \%}$ & $\mathbf{1 0 0 \%}$ \\
False Inclusion & $(6 / 6)$ & $(2 / 2)$ & $(2 / 2)$ & $(1 / 1)$ & $(1 / 1)$ \\
& $(648 / 3003)$ & $(268 / 528)$ & $(13 / 120)$ & $(11 / 120)$ & $16.7 \%$ \\
False Exclusion & 0 & 0 & 0 & 0 & 0 \\
& $(0 / 6)$ & $(0 / 2)$ & $(0 / 2)$ & $(0 / 1)$ & $(0 / 1)$ \\
\hline
\end{tabular}

Although the inkjet ink penetrates the paper fibers, paper contribution was insignificant for the analysis of inkjets by SEM-EDS since no elements in the Whatman 42 paper were present above the instrument's detection limit. Figure 19 shows an example of two discriminated samples (Inkjet 21 and 24) along with the Whatman 42 paper spectra. The figure also shows Inkjet 77 compared to its duplicate sample.

SEM imaging can potentially provide additional discrimination capabilities. Unfortunately, inkjet ink cannot be visualized with the scanning electron microscope since it contains no particulates; only the paper fibers are visible (Figure 20). Thus, SEM imaging is not a useful discrimination technique for inkjets. 


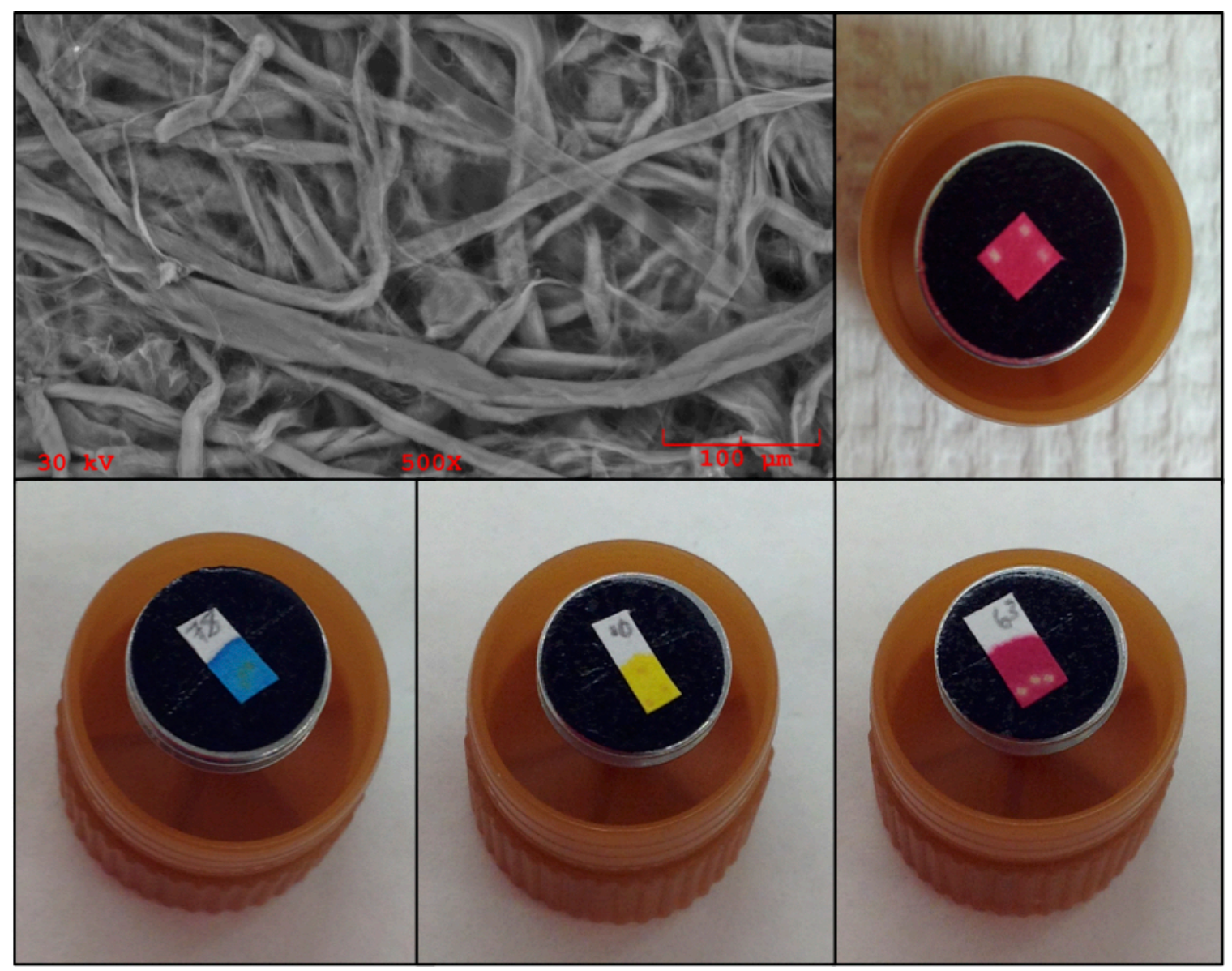

Figure 20 - SEM micrograph of inkjet (top left) and examples of discoloration of magenta (top and bottom right), cyan (bottom left), and yellow (bottom middle) inks

SEM-EDS is generally considered a nondestructive technique. However, it was observed that some of the inks showed discoloration in the areas that were sampled by SEM-EDS. The discoloration was particularly prominent for the magenta inkjets, though some cyan and yellow inks exhibited slight discoloration, as can be seen in Figure 20. Analysis was attempted at lower accelerating voltages $(25 \mathrm{kV}$ and $20 \mathrm{kV})$, however, discoloration was still observed, while sensitivity was greatly reduced. 


\subsubsection{Toner Inks}

SEM-EDS exhibited a surprisingly high (97.1\%) discrimination for the 76 toner samples analyzed. Of the pairs that could not be discriminated (82 out of 2850 comparison pairs), 18 were comparisons between pairs that were neither same brand nor same color, the majority of which were magenta versus either yellow or black toners. SEM-EDS was capable of detecting 13 elements in the toner set: $\mathrm{Al}, \mathrm{Ba}, \mathrm{Ca}, \mathrm{Cl} \mathrm{Cr}, \mathrm{Cu}, \mathrm{Fe}, \mathrm{S}, \mathrm{Si}, \mathrm{Sr}, \mathrm{Ti}$, $\mathrm{W}, \mathrm{Zn}$, and Zr. Manganese was also detected; however, this element was excluded because it was highly correlated with iron and provided no additional discrimination.

Since all toner samples originated from pure color cartridges, the discrimination for same-color comparison pairs was also calculated to assess the technique's capability of differentiating samples of the same color (Table 13). SEM-EDS provided good discrimination for each of the four colors $(>94 \%)$.

Table 13 - Discrimination (bolded), correct association (bolded), and false inclusion/exclusion rate for all SEM-EDS toner comparisons as well as for each individual color. Values in parentheses indicate either the number of pairwise comparisons or the fraction of discriminated/associated pairs.

\begin{tabular}{cccccc}
\hline & OVERALL & BLACK & CYAN & MAGENTA & YELLOW \\
\hline Number of & 76 & 39 & 12 & 12 & 13 \\
Samples & $(2850)$ & $(741)$ & $(66)$ & $(66)$ & $(78)$ \\
Number of & 25 & 16 & 4 & 4 & 1 \\
Duplicates & $(25)$ & $(16)$ & $(4)$ & $(4)$ & $(1)$ \\
Discrimination & $\mathbf{9 7 . 1 \%}$ & $\mathbf{9 4 . 2 \%}$ & $\mathbf{9 5 . 5 \%}$ & $\mathbf{9 5 . 5 \%}$ & $\mathbf{9 4 . 9 \%}$ \\
Correct & $(2768 / 2850)$ & $(698 / 741)$ & $(63 / 66)$ & $(63 / 66)$ & $(74 / 78)$ \\
Association & $\mathbf{1 0 0 \%}$ & $\mathbf{1 0 0 \%}$ & $\mathbf{1 0 0 \%}$ & $\mathbf{1 0 0 \%}$ & $\mathbf{1 0 0 \%}$ \\
False Inclusion & $(25 / 25)$ & $(2 / 2)$ & $(2 / 2)$ & $(1 / 1)$ & $(1 / 1)$ \\
& $2.9 \%$ & $5.3 \%$ & $4.5 \%$ & $4.5 \%$ & $5.1 \%$ \\
False Exclusion & $(82 / 2850)$ & $(39 / 741)$ & $(3 / 66)$ & $(3 / 66)$ & $(4 / 78)$ \\
& 0 & 0 & 0 & 0 & 0 \\
\hline
\end{tabular}


The correct association rate was calculated using duplicate samples. All 25 duplicate controls were correctly associated, resulting in no false exclusions. However, the number of duplicate samples is quite small, particularly for the CMY samples. A much larger sample set is necessary to estimate the true correct association.

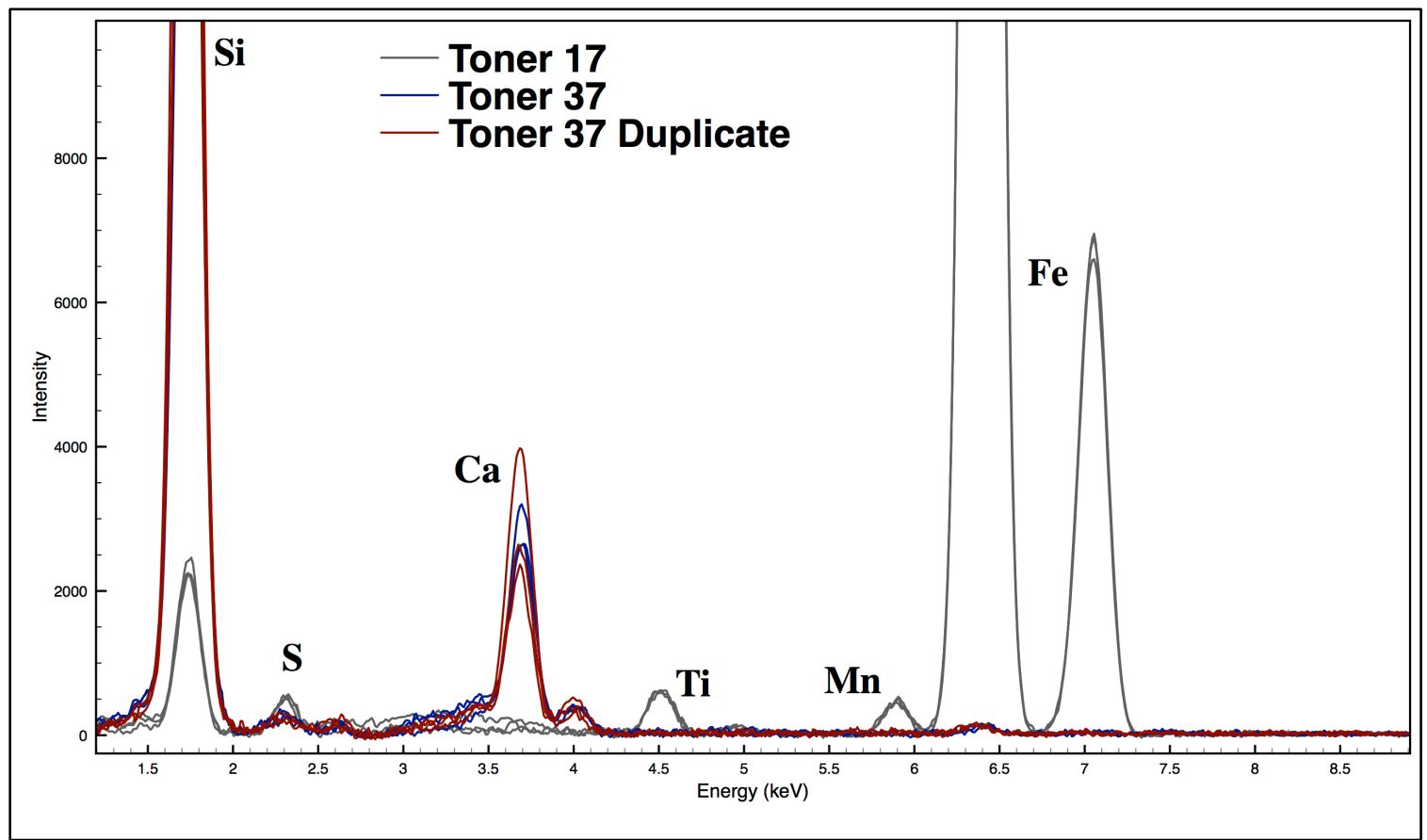

Figure 21 - Example of two toners that are distinguishable

Figure 21 shows an example of two ink samples that were discriminated as well as a duplicate sample. Toner 37 and its duplicate show good overlap, resulting in a correct association. Toner 17 is clearly distinguishable from Toner 37 on the basis of $\mathrm{Si}, \mathrm{Ca}, \mathrm{Ti}$, Mn (not used for discrimination), and Fe. The high Fe concentration suggests that this particular toner sample is a mono-component toner. Iron alone may be useful for the classification of mono-component versus two-component toners; the former typically 
have 30-70\% magnetite (which accounts for the high iron concentration), while the latter do not typically contain magnetite, but often contain higher levels of slow surface additives such as silicon. ${ }^{74}$ The paper contribution is omitted in Figure 21 for clarity; however, as was the case with inkjets, paper contribution was negligible since all samples were prepared on Whatman 42 paper.

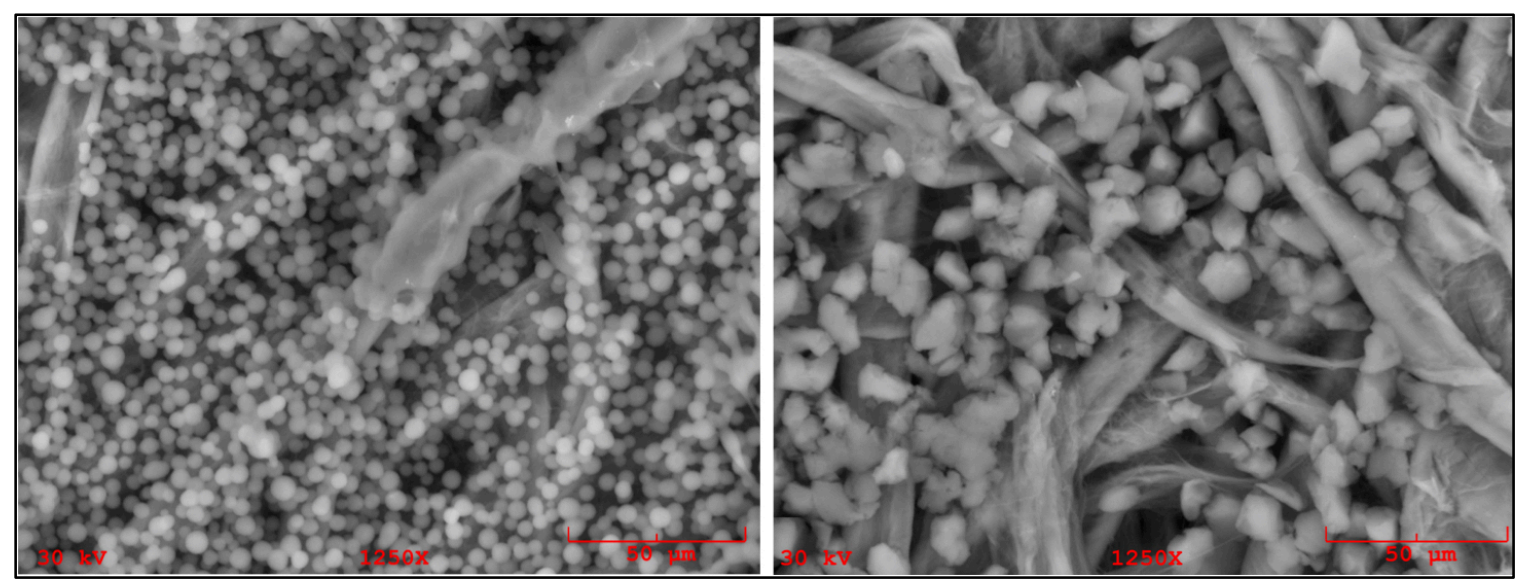

Figure 22 - SEM micrographs of two toners that were indistinguishable by their elemental profile, but distinguishable by their particle morphology

In the case of toners, the particle shape and size may provide an additional means of discrimination. In order to preserve the particle morphology, the toner samples were prepared slightly differently than described earlier: the ink powder was pressed lightly on top of the Whatman 42 paper using a plastic micropipette tip. The sample preparation method described above was inappropriate for the other instrumental techniques used in the ink project, particularly the laser ablation techniques (discussed in later sections) because the laser shots scattered the toner particles, which significantly affected the sensitivity. Therefore, the manual deposition method that involved melting the toner onto the Whatman 42 paper was developed for the other instrumental techniques. Of the 82 
pairs that could not be differentiated by elemental analysis, 10 pairs were discriminated on the basis of particle morphology, increasing the percent discrimination slightly to $97.5 \%$. Figure 22 shows the SEM micrograph for a pair of black toners (different brands) that was indistinguishable by their elemental profile, but readily distinguishable by particle morphology. Thus, particle morphology may be useful as a preliminary classification tool for toners.

\subsubsection{Offset Inks}

The offset samples presented the greatest challenge for SEM-EDS analysis as a result of paper contribution. Despite the fact that more elements were detected in the ink compared to toners and inkjets, discrimination was relatively poor (80.3\%). Like inkjet ink, the offset ink is embedded into the paper substrate. However, whereas the inkjet samples were deposited on Whatman 42, most offset samples were printed on regular office paper, which had several elements (Table 11) that were detectable by SEM-EDS. Calcium was particularly problematic, since it was present at high concentration in the paper (Figure 23) making it difficult to determine whether part of the calcium signal could be attributed to the ink. An effort was made to scrape the offset ink from the paper in order to remove the paper interferences and ascertain which elements were present solely in the ink. However, the attempts were unsuccessful as the ink was deeply embedded within the paper fibers. Therefore, calcium was eliminated from the element menu altogether. All other elements were only included for discrimination if their signal was greater than that of the paper blank signal. A total of 20 duplicate samples were analyzed, all of which were correctly associated by spectral overlay. 


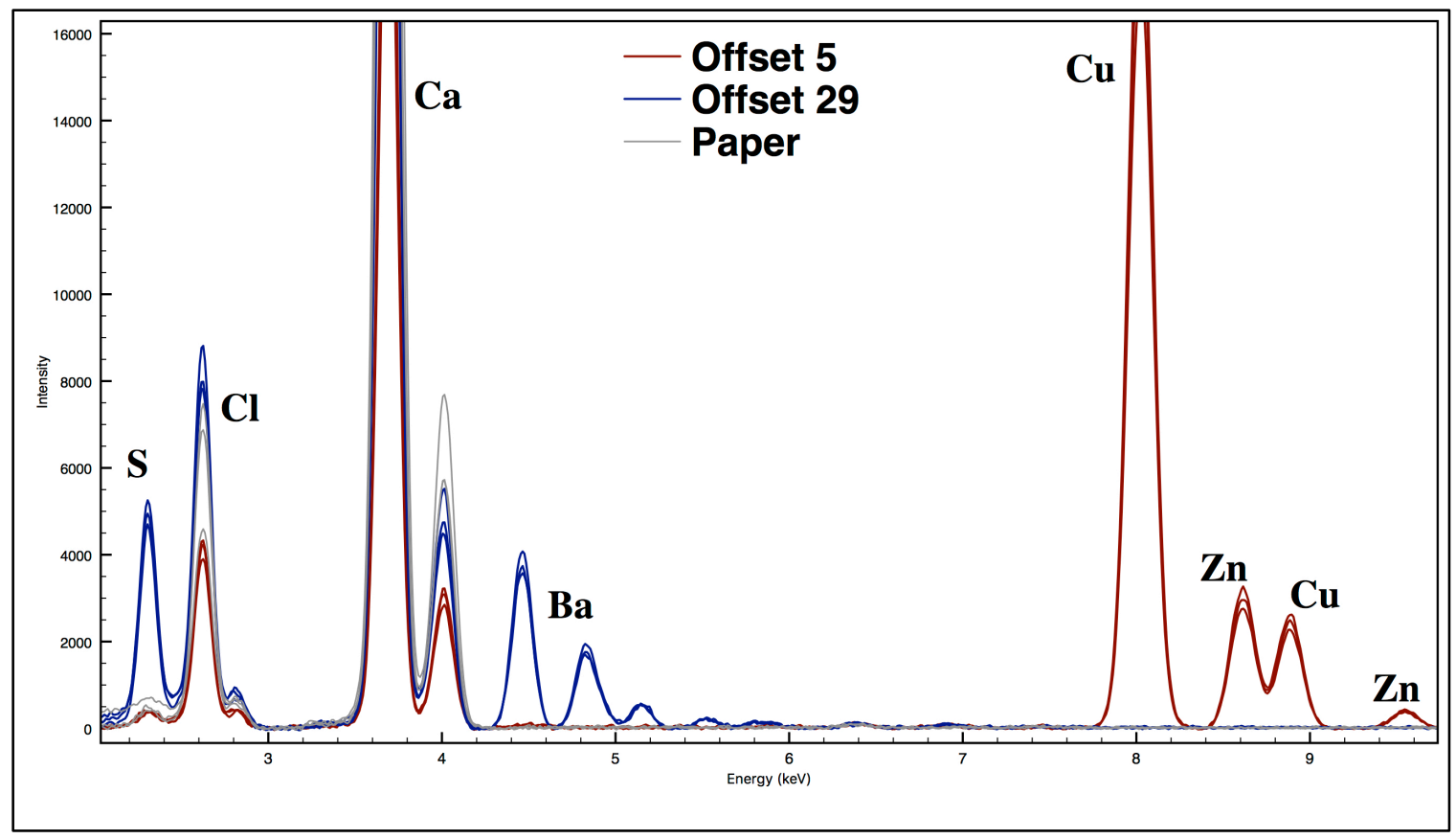

Figure 23 - Example of two offset inks that are easily distinguished; the paper blank is also included to demonstrate the high concentration of calcium

Figure 15 shows the spectra of two offset inks that were easily distinguished using the concentration of $\mathrm{S}, \mathrm{Ba}, \mathrm{Cu}$, and $\mathrm{Zn}$. However, these two inks are different colors (brown versus orange) and can be distinguished visually. Since offset inks include a variety of colors, it was not feasible to estimate the percent discrimination for individual colors.

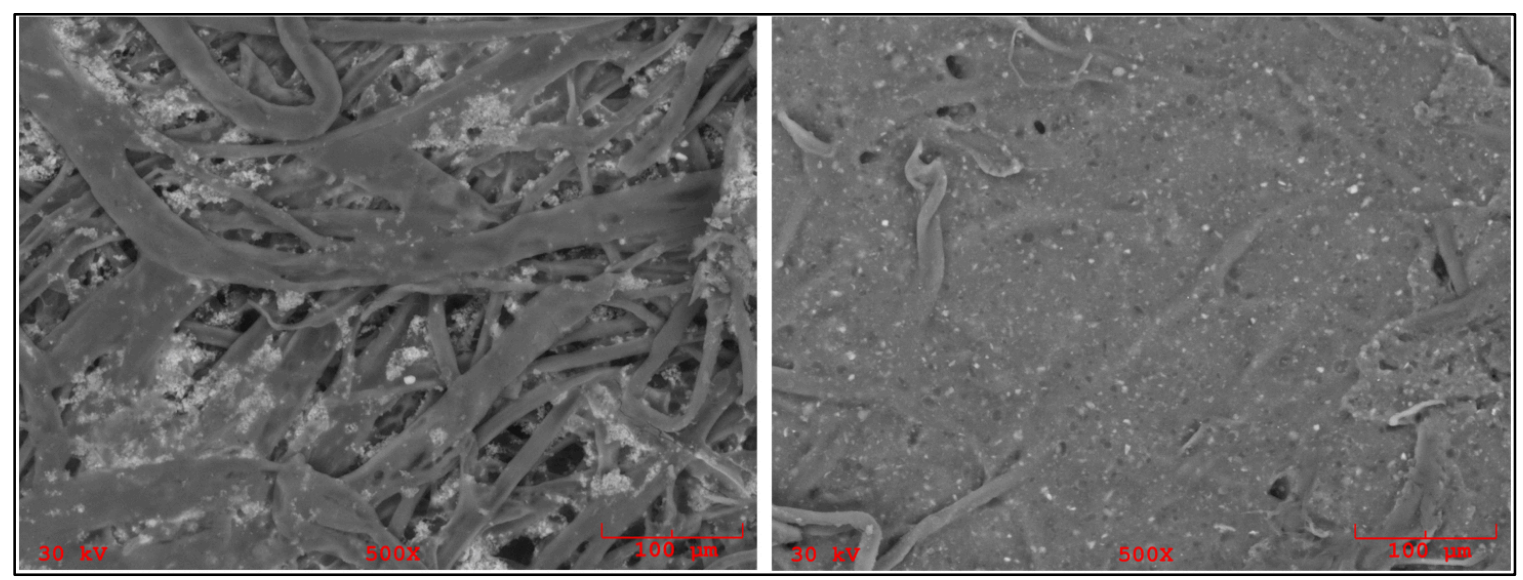

Figure 24 - SEM micrographs of two offset samples 
SEM imaging revealed few morphological differences between the offset samples (Figure 24). Moreover, those pairs that exhibited differences were readily distinguishable by their elemental profile, thus SEM imaging did not provide any additional information.

\subsubsection{Intaglio Inks}

The highest discrimination (98.2\%) was observed for the intaglio because of the large number of detectable elements in the ink formulations: $\mathrm{Al}, \mathrm{Ba}, \mathrm{Br}, \mathrm{Ca}, \mathrm{Cl}, \mathrm{Co}, \mathrm{Cr}, \mathrm{Cu}, \mathrm{F}$, Fe, K, Mg, P, S, Si, Ti, and Zn. Of the 64 pairs that were not discriminated, 28 were comparisons between banknotes from the same country. All six duplicate controls were correctly associated, resulting in no false exclusions.

Although there were several elements detected in the paper for the intaglio samples, paper contribution was irrelevant since the ink layer thickness of the intaglio samples was in the range of $19-45 \mu \mathrm{m}$ and the SEM electron beam does not typically penetrate more than $\sim 5 \mu \mathrm{m}^{2}$ into the sample. ${ }^{121}$ Figure 25 shows the EDS spectra for three different intaglio inks (Intaglio 1, 4, and 58) that were easily distinguished on the basis of several elements. Intaglio 58 was a fairly unique sample that contained metallic flakes (right SEM micrograph in Figure 26), giving the ink a golden shimmer. Intaglio 1 and 4 were both a dark green color, but originated from banknotes of different countries; this pair was easily distinguished by $\mathrm{Cl}, \mathrm{Ti}$, and $\mathrm{Br}$. Like offsets, intaglio inks included a variety of colors. It was therefore impractical to estimate the discrimination for each individual color. 


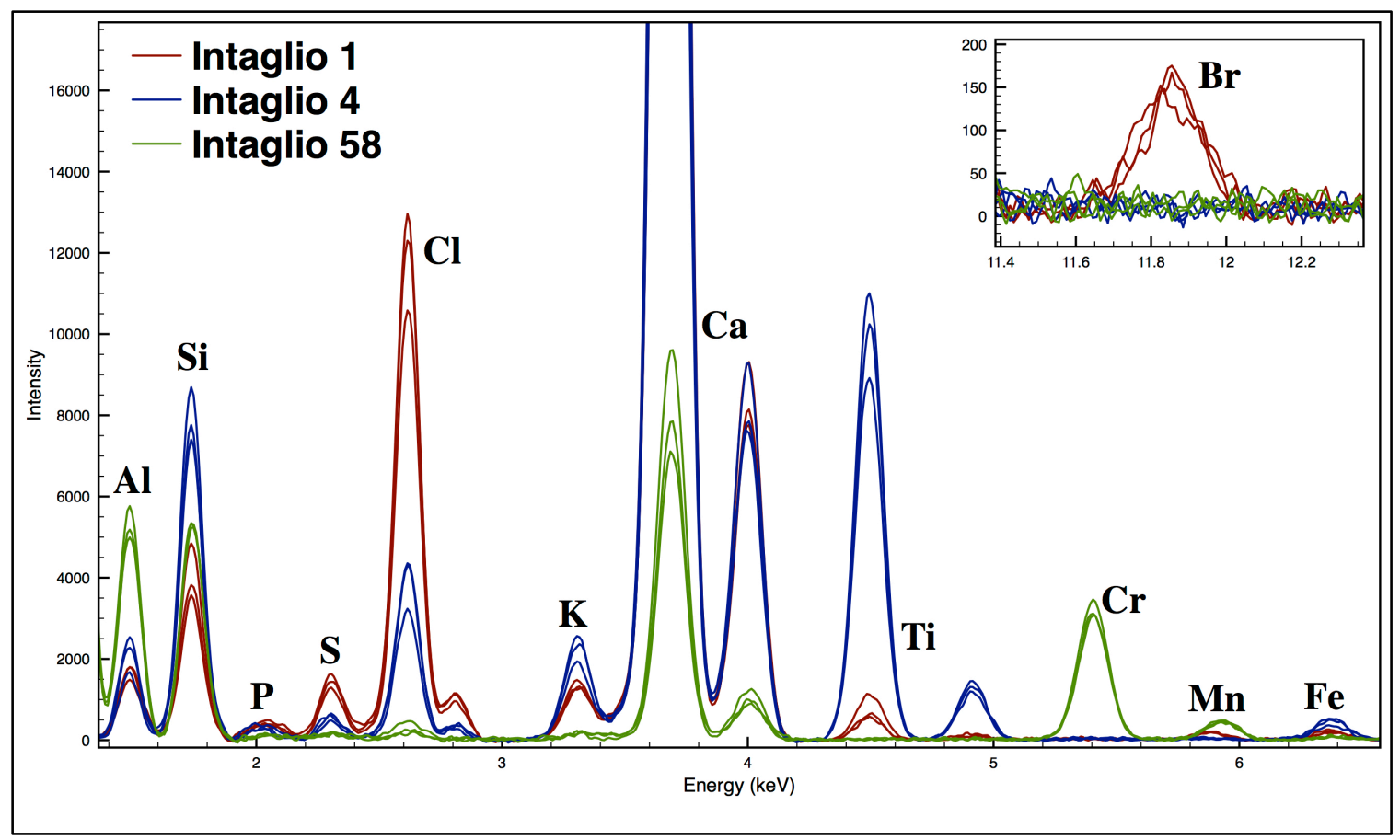

Figure 25 - Examples of three intaglio inks that were easily distinguished

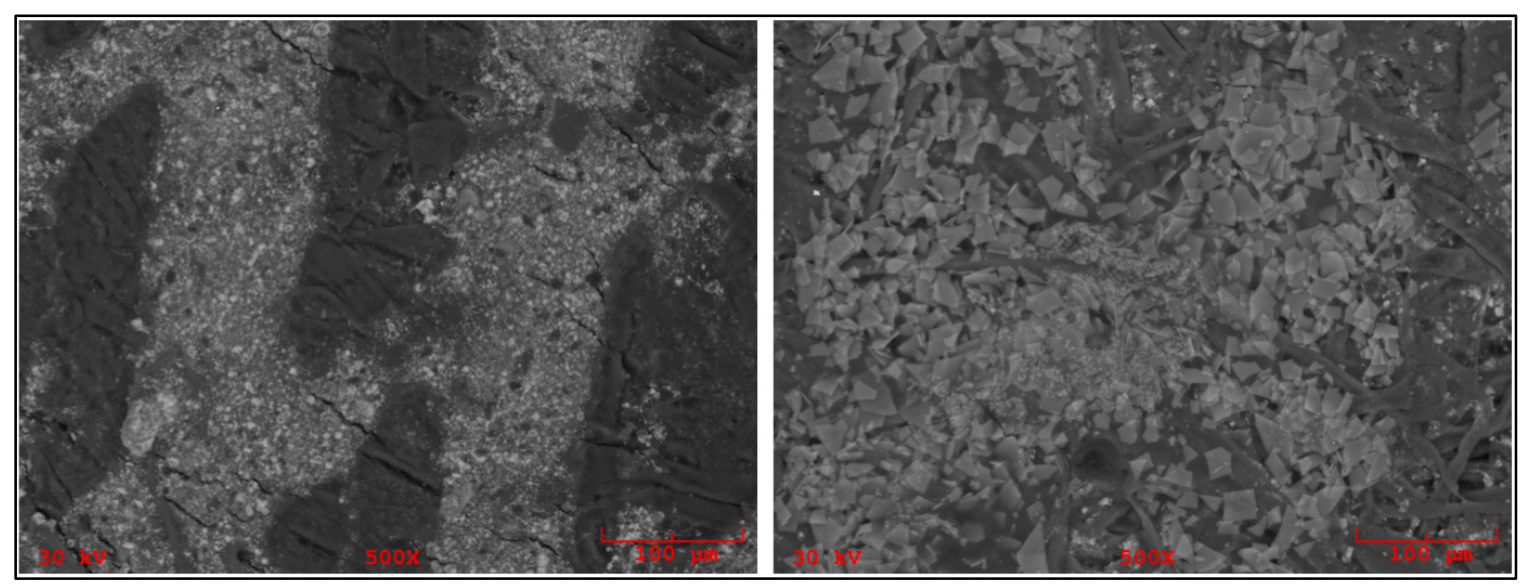

Figure 26 - SEM micrographs of two intaglio samples, one with particulates (left) and the other with metallic flakes (right)

Figure 26 shows the SEM micrograph of two intaglio samples exhibiting different morphologies (one with particles and the other with metallic flakes). However, this pair 
was readily distinguishable by their elemental profile. Overall, SEM imaging provided no additional information for the discrimination of intaglio.

\subsection{Comparison of SEM-EDS and LA-ICP-MS Results}

The analysis of all printing inks using LA-ICP-MS was conducted by another graduate student, Kiran Subedi. The present section compares to compare the SEM-EDS results presented earlier with a more sensitive elemental technique used herein. The analyses were conducted on a quadrupole ELAN DRC II (Perkin Elmer LAS, Shelton CT USA), coupled to a $213 \mathrm{~nm}$ ns-Nd:YAG laser (NW UP213, New Wave, CA). A $266 \mathrm{~nm}$ nsNd:YAG laser coupled to the same quadrupole was also used for the analysis of duplicate samples (J200, Applied Spectra, CA). A full mass spectrum from m/z 7 to m/z $238\left({ }^{7} \mathrm{Li}\right.$ to $\left.{ }^{238} \mathrm{U}\right)$ was collected using a line ablation mode. The optimized parameters for each ink type are listed in Table 14.

The mass spectra obtained by LA-ICP-MS analysis were normalized to the sum of the intensities for the entire spectrum. The elemental composition of the ink was subtracted from the paper contribution, if any. As was the case for SEM-EDS, pairwise comparisons were conducted using spectral overlay. 
Table 14 - Optimized LA-ICP-MS parameters for the analysis of printing inks; elements detected $(\mathrm{SNR} \geq 3)$ in the ink and paper are also reported

\begin{tabular}{|c|c|c|c|c|}
\hline & INKJET & TONER & OFFSET & INTAGLIO \\
\hline $\begin{array}{c}\text { Laser } \\
\text { Wavelength } \\
\text { (nm) }\end{array}$ & $213 / 266$ & $213 / 266$ & $213 / 266$ & 213 / NA \\
\hline Spot Size $(\mu \mathrm{m})$ & $190 / 200$ & $190 / 200$ & $190 / 200$ & 190 / NA \\
\hline $\begin{array}{l}\text { Laser Energy } \\
(\%)\end{array}$ & $40 / 0.5$ & $40 / 0.5$ & $25 / 0.2$ & 35 / NA \\
\hline $\begin{array}{l}\text { Stage Speed } \\
\qquad(\mu \mathrm{m} / \mathrm{s})\end{array}$ & $25 / 15$ & $25 / 15$ & $25 / 15$ & $25 / \mathrm{NA}$ \\
\hline $\begin{array}{l}\text { Repetition } \\
\text { Rate (Hz) }\end{array}$ & $10 / 5$ & $10 / 5$ & $10 / 5$ & 10 / NA \\
\hline $\begin{array}{c}\text { Ablation } \\
\text { Length }(\mu \mathrm{m})\end{array}$ & $950 / 1000$ & $950 / 1000$ & $800 / 1000$ & 800 / NA \\
\hline $\begin{array}{l}\text { Helium Flow } \\
\text { Rate (L/s) }\end{array}$ & $0.9 / 0.6$ & $0.9 / 0.6$ & $0.9 / 0.6$ & 0.9 / NA \\
\hline $\begin{array}{l}\text { Elements } \\
\text { Detected in } \\
\text { Ink }\end{array}$ & $\begin{array}{c}\mathrm{Al}, \mathrm{B}, \mathrm{Ba}, \mathrm{Cu}, \\
\mathrm{Hf}, \mathrm{K}, \mathrm{Li}, \mathrm{Mg}, \\
\mathrm{Na}, \mathrm{S}, \mathrm{Sn}, \mathrm{Zr}\end{array}$ & $\begin{array}{c}\mathrm{Al}, \mathrm{Ba}, \mathrm{Ca}, \mathrm{Cr}, \\
\mathrm{Cu}, \mathrm{Fe}, \mathrm{Hf}, \mathrm{K}, \\
\mathrm{Mg}, \mathrm{Mn}, \mathrm{Mo}, \\
\mathrm{Na}, \mathrm{Nb}, \mathrm{Sb}, \mathrm{Si}, \\
\mathrm{Sn}, \mathrm{Sr}, \mathrm{Ti}, \mathrm{W}, \\
\mathrm{Zn}, \mathrm{Zr}\end{array}$ & $\begin{array}{c}\mathrm{Al}, \mathrm{Ag}, \mathrm{Ba}, \mathrm{Ca}, \\
\mathrm{Ce}, \mathrm{Co}, \mathrm{Cu}, \mathrm{K}, \\
\mathrm{Mg}, \mathrm{Mn}, \mathrm{Mo}, \\
\mathrm{Na}, \mathrm{Nb}, \mathrm{Rh}, \mathrm{S}, \\
\mathrm{Si}, \mathrm{Sn}, \mathrm{Sr}, \mathrm{Ti} \\
\text { W, Zn, Zr }\end{array}$ & $\begin{array}{c}\mathrm{Al}, \mathrm{Ba}, \mathrm{Bi}, \mathrm{Ca}, \\
\mathrm{Co}, \mathrm{Cu}, \mathrm{Fe}, \mathrm{Hf}, \\
\mathrm{K}, \mathrm{Mg}, \mathrm{Mn}, \\
\mathrm{Mo}, \mathrm{Na}, \mathrm{Nb}, \\
\mathrm{Pb}, \mathrm{Sb}, \mathrm{Si}, \mathrm{Sn}, \\
\mathrm{Sr}, \mathrm{Ti}, \mathrm{W}, \mathrm{Zn}, \\
\mathrm{Zr}\end{array}$ \\
\hline $\begin{array}{c}\text { Elements } \\
\text { Detected in } \\
\text { Paper }\end{array}$ & $\begin{array}{c}\mathrm{Ba}, \mathrm{Ca}, \mathrm{Na}, \mathrm{Si}, \\
\mathrm{Zn}\end{array}$ & $\begin{array}{c}\mathrm{Ba}, \mathrm{Ca}, \mathrm{Na}, \mathrm{Si}, \\
\mathrm{Zn}\end{array}$ & $\begin{array}{c}\mathrm{Al}, \mathrm{Ba}, \mathrm{Ca}, \mathrm{Mg}, \\
\mathrm{Na}, \mathrm{Ti}, \mathrm{Si}, \mathrm{Sr} \\
\mathrm{Eu}, \mathrm{Zn}\end{array}$ & $\begin{array}{l}\mathrm{Co}, \mathrm{Cu}, \mathrm{Eu}, \mathrm{Fe}, \\
\mathrm{Hg}, \mathrm{Hf}, \mathrm{Ho}, \mathrm{K}, \\
\mathrm{Na}, \mathrm{Pb}, \mathrm{Sn}, \mathrm{Sm}, \\
\mathrm{Sr}, \mathrm{Ti}, \mathrm{U}, \mathrm{W}, \mathrm{Zr}\end{array}$ \\
\hline
\end{tabular}

\subsubsection{Inkjet Inks}

LA-ICP-MS performed better than SEM-EDS for the analysis of inkjets, providing a discrimination of $99.6 \%$ (2992 out of 3003 comparison pairs) and a false inclusion rate of $0.4 \%$. Because of its superior sensitivity, LA-ICP-MS was capable of detecting a greater number of elements: 12 versus just five detectable elements using SEM-EDS. Of the 11 pairs that were not discriminated, eight were same-brand comparisons; from the remaining three pairs, one pair was a same-color comparison and the other two were 
comparisons between magenta and yellow inks. However, SEM-EDS can provide some complementary information for the analysis of inkjets. Six of the 11 pairs that were not discriminated by LA-ICP-MS were differentiated by SEM-EDS on the basis of sulfur and/or chlorine.
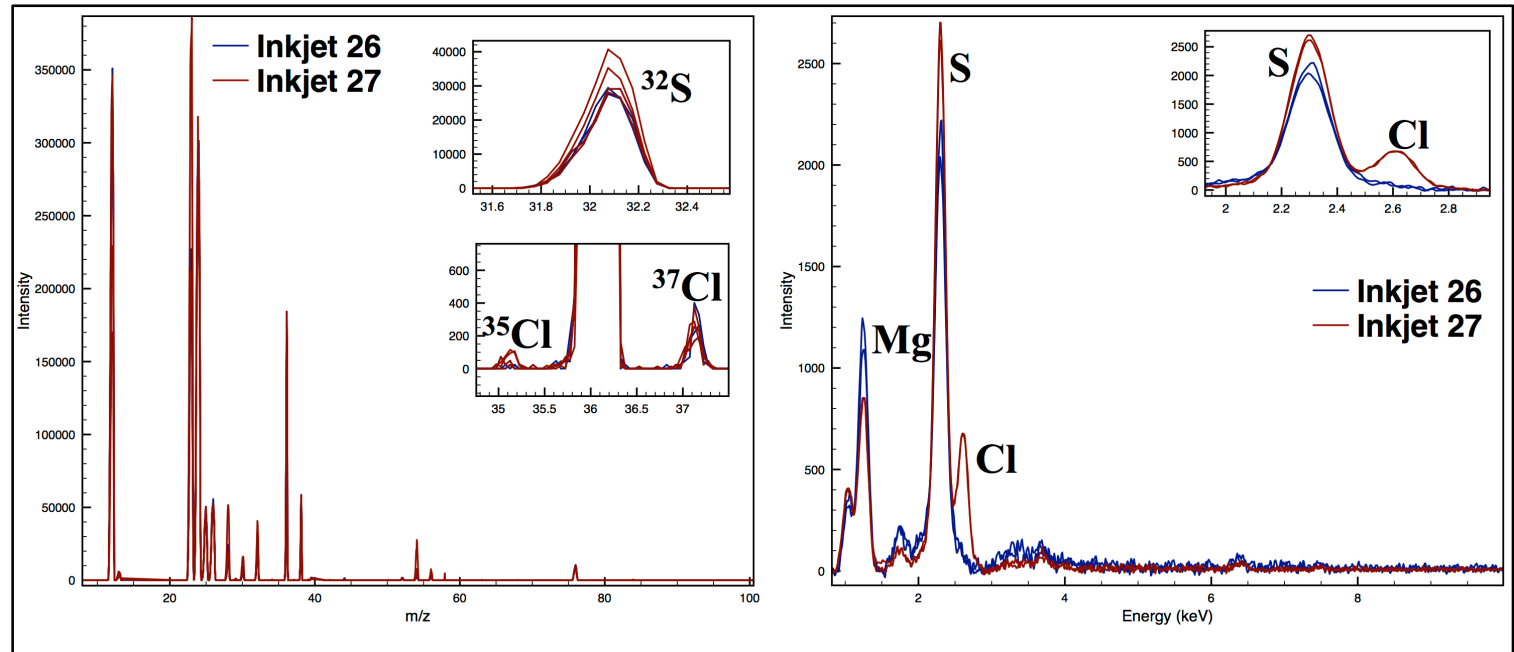

Figure 27 - Comparison of two inkjet samples that were indistinguishable by LA-ICP-MS (left), but distinguishable by SEM-EDS (right) on the basis of the sulfur and chlorine content

Figure 27 shows the spectra, and a close-up of the chlorine region, for two inkjets that were indistinguishable using LA-ICP-MS due to polyatomic interferences $\left({ }^{16} \mathrm{O}^{18} \mathrm{O}^{1} \mathrm{H}^{+}\right.$, ${ }^{34} \mathrm{~S}^{1} \mathrm{H}^{+},{ }^{36} \mathrm{Ar}^{1} \mathrm{H}^{+},{ }^{36} \mathrm{~S}^{1} \mathrm{H}^{+}$), but distinguishable using SEM-EDS on the basis of the chlorine and sulfur contribution. ${ }^{144}$ Apart from polyatomic interferences, LA-ICP-MS detection of sulfur and chlorine is challenging since both elements are difficult to ionize because of their high ionization energy. ${ }^{123}$ 
Table 15 - Discrimination (bolded), correct association (bolded), and false inclusion/exclusion rate for all LA-ICP-MS inkjet comparisons as well as for each individual color. Values in parentheses indicate either the number of pairwise comparisons or the fraction of discriminated/associated pairs.

\begin{tabular}{|c|c|c|c|c|c|}
\hline & OVERALL & BLACK & CYAN & MAGENTA & YELLOW \\
\hline $\begin{array}{l}\text { Number of } \\
\text { Samples }\end{array}$ & $\begin{array}{c}78 \\
(3003)\end{array}$ & $\begin{array}{c}33 \\
(528)\end{array}$ & $\begin{array}{c}16 \\
(120)\end{array}$ & $\begin{array}{c}16 \\
(120)\end{array}$ & $\begin{array}{c}13 \\
(78)\end{array}$ \\
\hline $\begin{array}{l}\text { Number of } \\
\text { Duplicates }\end{array}$ & $\begin{array}{c}3 \\
(3)\end{array}$ & NA & $\begin{array}{c}3 \\
(3)\end{array}$ & NA & NA \\
\hline Discrimination & $\begin{array}{c}\mathbf{9 9 . 6 \%} \\
(2992 / 3003)\end{array}$ & $\begin{array}{c}\mathbf{9 9 . 8 \%} \\
(527 / 528)\end{array}$ & $\begin{array}{c}\mathbf{1 0 0 \%} \\
(120 / 120)\end{array}$ & $\begin{array}{c}\mathbf{9 9 . 2 \%} \\
(119 / 120)\end{array}$ & $\begin{array}{l}\mathbf{9 8 . 7 \%} \\
(77 / 78)\end{array}$ \\
\hline $\begin{array}{c}\text { Correct } \\
\text { Association }\end{array}$ & $\begin{array}{c}100 \% \\
(6 / 6)\end{array}$ & NA & $\begin{array}{c}100 \% \\
(3 / 3)\end{array}$ & NA & NA \\
\hline False Inclusion & $\begin{array}{c}0.4 \% \\
(11 / 3003)\end{array}$ & $\begin{array}{c}0.2 \% \\
(1 / 528)\end{array}$ & $\begin{array}{c}0 \\
(0 / 120)\end{array}$ & $\begin{array}{l}0.8 \% \\
(1 / 20)\end{array}$ & $\begin{array}{l}0.3 \% \\
(1 / 78)\end{array}$ \\
\hline False Exclusion & $\begin{array}{c}0 \\
(0 / 3) \\
\end{array}$ & NA & NA & $\begin{array}{c}0 \\
(0 / 3) \\
\end{array}$ & NA \\
\hline
\end{tabular}

Table 15 shows the discrimination, correct association, and false inclusion and exclusion rate for all inkjets as well as for each individual color (CMYK). All colors resulted in greater than 99\% discrimination, highlighting the excellent discrimination capabilities for the analysis of toners. While black inkjets were especially problematic for SEM-EDS, LA-ICP-MS easily distinguished all but one pair. Moreover, all 3 duplicate controls were correctly associated using LA-ICP-MS, resulting in no false exclusions. It should be noted, however, that all 3 duplicates originated from the same cyan specimen (Inkjet 25). A larger set of duplicates originating from different sources is required to truly evaluate the correct association rate. 


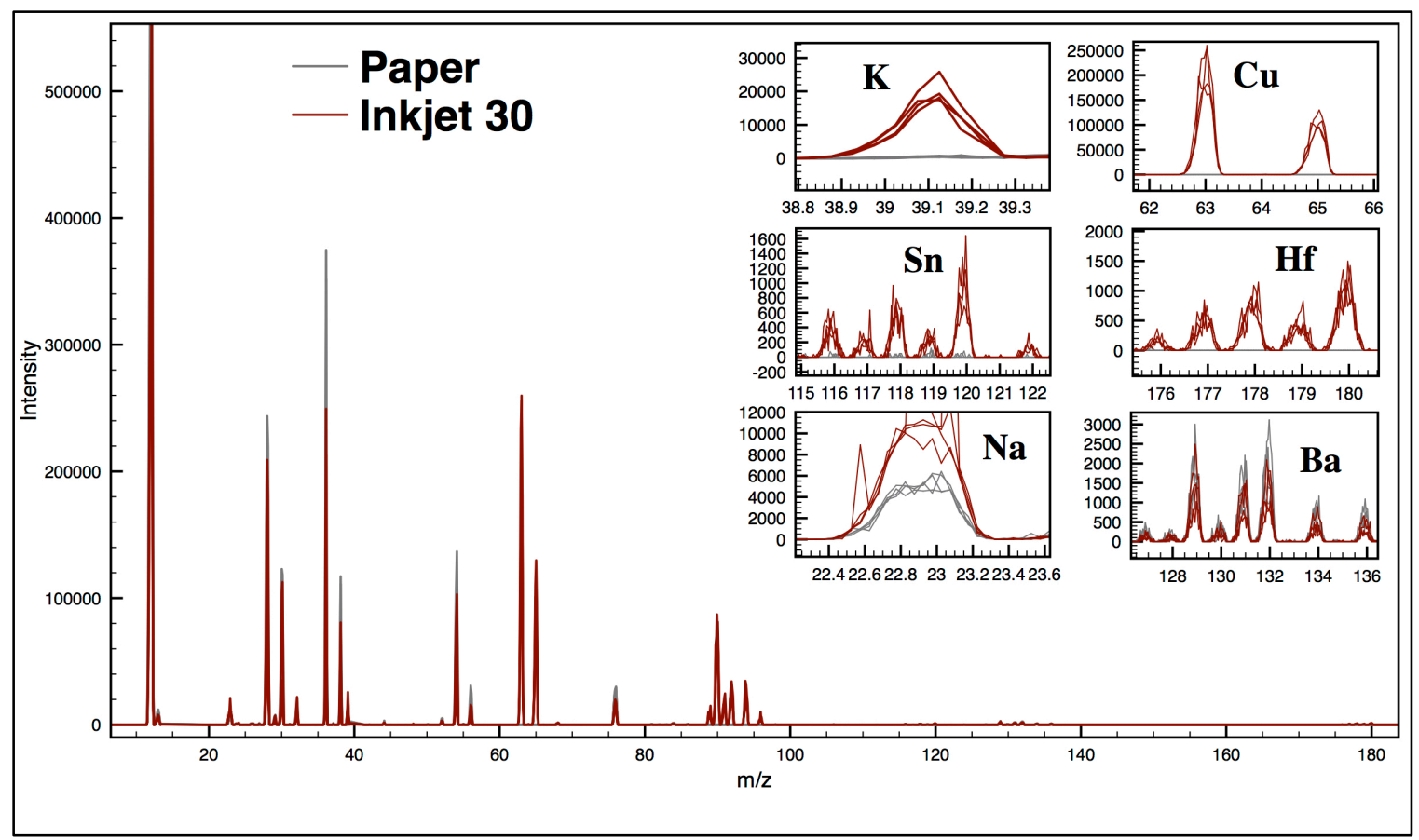

Figure 28 - Example of inkjet and paper mass spectra

Unlike SEM-EDS, LA-ICP-MS was capable of detecting 5 elements in the Whatman 42 paper: $\mathrm{Ba}, \mathrm{Ca}, \mathrm{Na}, \mathrm{Si}$, and $\mathrm{Zn}$. As such, only elements that were present at higher concentrations in the ink compared to the paper blank were included as discriminating elements. Figure 28 shows an example of an inkjet and a paper blank mass spectrum. In this example, several elements were detectable in the ink, but not the paper $(\mathrm{K}, \mathrm{Cu}, \mathrm{Sn}$, Hf). Other elements, such as $\mathrm{Ba}$, were detected in the ink and paper at approximately equal concentrations and thus excluded for discrimination purposes. Finally, some elements, such as $\mathrm{Na}$, were present at higher concentrations in the ink compared to the paper and thus were included for discrimination. 


\subsubsection{Toner Inks}

As with inkjets, LA-ICP-MS provided superior discrimination (99.1\%) for toners as a result of the technique's higher sensitivity, which allowed for the detection of more elements (21 elements compared to 14 elements by SEM-EDS). Of the 25 pairs that were indistinguishable by LA-ICP-MS, only four were samples that did not originate from the same brand and/or were the same color; as observed with SEM-EDS, these four pairs were comparisons between magenta and yellow toners. As mentioned previously, SEMEDS can provide complementary information for the analysis of toner inks: 14 of the 25 pairs that were not discriminated by LA-ICP-MS were differentiated by SEM-EDS on the basis of $\mathrm{Fe}, \mathrm{Zn}, \mathrm{Ca}, \mathrm{S}, \mathrm{Cl}$ and/or Si, elements that are difficult to analyze by LA-ICP-MS due to the presence of polyatomic interferences and/or high ionization potential.

Figure 29 shows the spectra for a pair of toners that was not differentiated by LA-ICPMS, but was differentiated by SEM-EDS on the basis of the silicon and zinc content. Silicon suffers from several nitrogen, carbon, and oxide polyatomic interferences $\left({ }^{14} \mathrm{~N}^{15} \mathrm{~N}^{+},{ }^{14} \mathrm{~N}_{2}{ }^{1} \mathrm{H}^{+},{ }^{13} \mathrm{C}^{16} \mathrm{O}^{+},{ }^{12} \mathrm{C}^{17} \mathrm{O}^{+},{ }^{12} \mathrm{C}^{16} \mathrm{O}^{1} \mathrm{H}^{+}\right)$, while zinc suffers from several sulfur

and oxide interferences $\left({ }^{36} \mathrm{~S}^{16} \mathrm{O}_{2}{ }^{+},{ }^{34} \mathrm{~S}^{16} \mathrm{O}^{18} \mathrm{O}^{+},{ }^{40} \mathrm{Ar}^{14} \mathrm{~N}_{2}{ }^{+},{ }^{35} \mathrm{Cl}^{16} \mathrm{O}^{17} \mathrm{O}^{+},{ }^{34} \mathrm{~S}_{2}{ }^{+},{ }^{36} \mathrm{Ar}^{32} \mathrm{~S}^{+}\right.$, $\left.{ }^{34} \mathrm{~S}^{17} \mathrm{O}_{2}^{+},{ }^{33} \mathrm{~S}^{17} \mathrm{O}^{18} \mathrm{O}^{+},{ }^{32} \mathrm{~S}^{18} \mathrm{O}_{2}+,{ }^{32} \mathrm{~S}^{36} \mathrm{~S}^{+}\right) \cdot{ }^{144}$

Although the toner ink thickness was in the range of $20-95 \mu \mathrm{m}$, paper contribution was relevant since the laser penetrates deeply enough to remove both ink and paper. Thus, only elements that were present at higher concentrations in the ink compared to the paper blank were included as discriminating elements. 


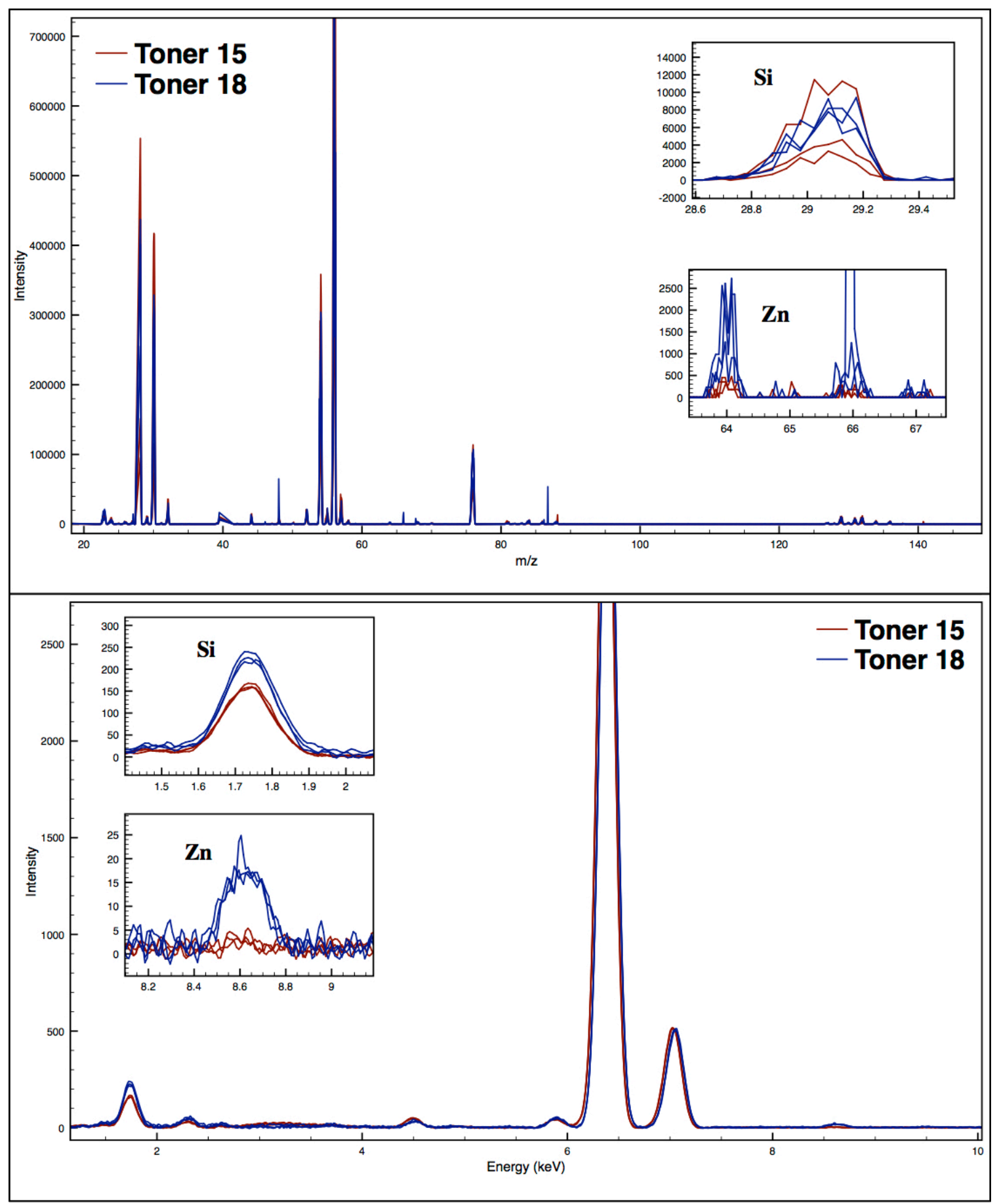

Figure 29 - Comparison of two toner samples that were indistinguishable by LA-ICP-MS (top), but distinguishable by SEM-EDS (bottom) on the basis of the silicon and zinc 
Table 16 shows the discrimination, correct association, and false inclusion and exclusion rate for all inkjets as well as for each individual color (CMYK). All colors resulted in greater than 99\% discrimination, highlighting the excellent discrimination capabilities for the analysis of inkjets.

Table 16 - Discrimination (bolded), correct association (bolded), and false inclusion/exclusion rate for all LA-ICP-MS toner comparisons as well as for each individual color. Values in parentheses indicate either the number of pairwise comparisons or the fraction of discriminated/associated pairs.

\begin{tabular}{cccccc}
\hline & OVERALL & BLACK & CYAN & MAGENTA & YELLOW \\
\hline Number of & 76 & 39 & 12 & 12 & 13 \\
Samples & $(2850)$ & $(741)$ & $(66)$ & $(66)$ & $(78)$ \\
Number of & 24 & 15 & 5 & 3 & 1 \\
Duplicates & $(26)$ & $(17)$ & $(5)$ & $(3)$ & $(1)$ \\
Discrimination & $\mathbf{9 9 . 1 \%}$ & $\mathbf{9 7 . 6 \%}$ & $\mathbf{9 8 . 5 \%}$ & $\mathbf{1 0 0 \%}$ & $\mathbf{9 7 . 4 \%}$ \\
Correct & $(2825 / 2850)$ & $(723 / 741)$ & $(65 / 66)$ & $(66 / 66)$ & $(76 / 78)$ \\
Association & $\mathbf{9 2 . 3 \%}$ & $\mathbf{1 0 0 \%}$ & $\mathbf{1 0 0 \%}$ & $\mathbf{3 3 . 3 \%}$ & $\mathbf{1 0 0 \%}$ \\
False Inclusion & $(24 / 26)$ & $(17 / 17)$ & $(5 / 5)$ & $(1 / 3)$ & $(1 / 1)$ \\
& $(25 / 3003)$ & $2.4 \%$ & $1.5 \%$ & 0 & $2.6 \%$ \\
False Exclusion & $7.7 \%$ & 0 & 0 & $66 \% 7 \%$ & 0 \\
& $(2 / 26)$ & $(0 / 17)$ & $(0 / 5)$ & $(2 / 3)$ & $(0 / 1)$ \\
\hline
\end{tabular}

Four quality controls and 20 duplicates were analyzed to assess the correct association. Thus, the total number of same-source comparisons is $26(4 \times 3 \div 2+20)$. A quality control is a single sample (toner 37) that is analyzed multiple times, while a duplicate is a sample that is analyzed a second time some time after the original sample was analyzed. Essentially, a quality control is a duplicate that has been analyzed more than twice; however, quality controls are useful for assessing intra-day and/or inter-day variation over a length of time. Two duplicate pairs were falsely excluded. However, these two 
pairs were analyzed by different laser ablation units as part of an inter-instrumental variation assessment and only differed in intensity for one element $(\mathrm{Mg})$.

\subsubsection{Offset Inks}

Offset inks presented a challenge for SEM-EDS since the ink is embedded into the paper substrate, which led to a convoluted signal for the ink and paper. Despite this issue, LAICP-MS was capable of discriminating $99.8 \%$ of the offset inks. Although a greater number of elements were detected in the paper substrate using LA-ICP-MS, the ink signal was typically greater than the paper signal. Four of the six false exclusions were comparisons between pairs with the same manufacturer. The remaining two pairs originated from different manufacturers and had a different color: Offset 47 with Offset 50 (green visa sample versus orange passport sample) and Offset 18 with Offset 65 (cyan sample from Sunchemical versus black sample from Unibrilliant).

SEM-EDS was able to discriminate 4 of the 6 offset pairs that were indistinguishable by LA-ICP-MS, often on the basis of the problematic elements $\mathrm{Si}, \mathrm{Cl}$, and/or Fe. Figure 30 shows one offset pair that was indistinguishable using LA-ICP-MS, but distinguishable using SEM-EDS. 


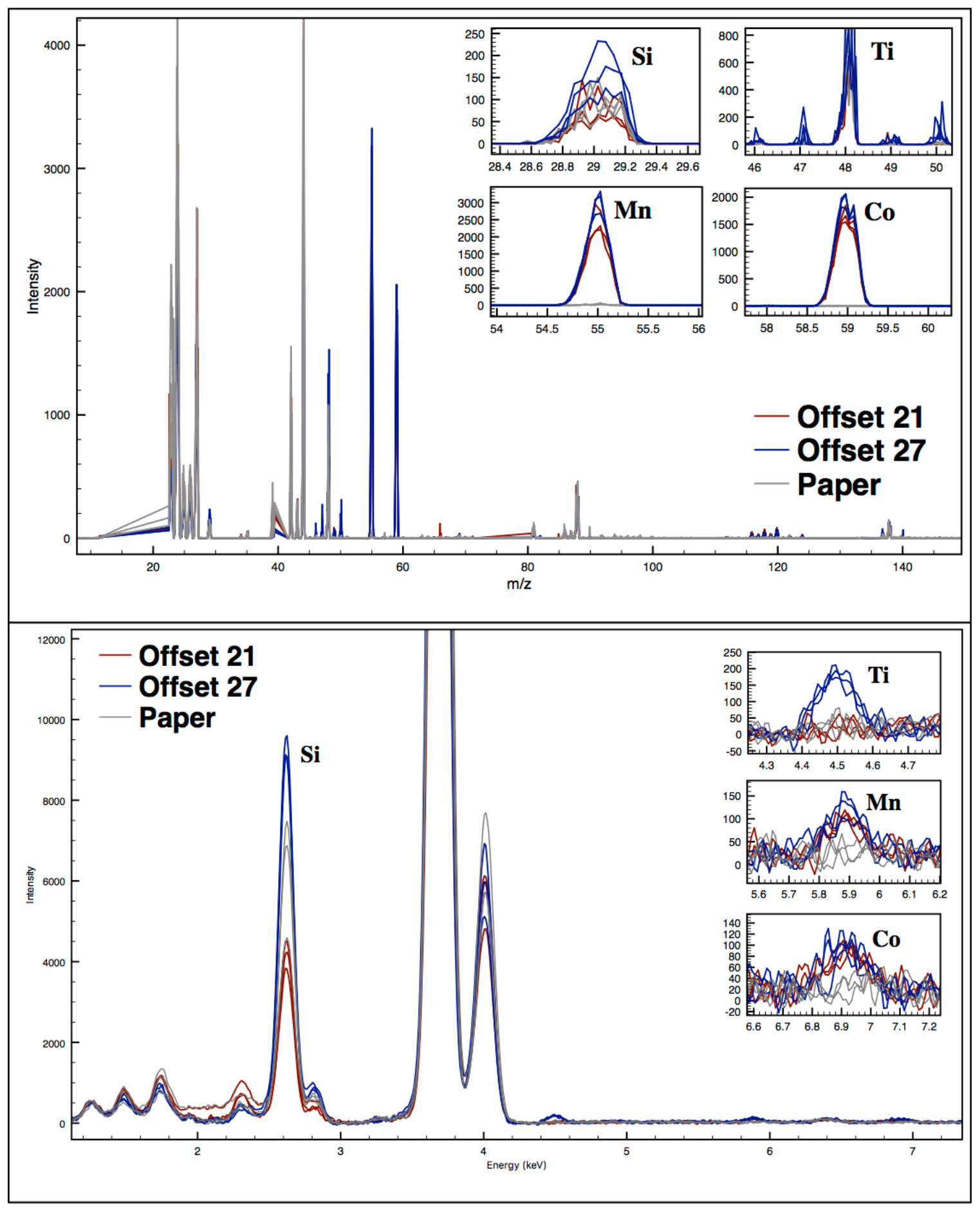

Figure 30 - Comparison of two offset samples that were indistinguishable by LA-ICP-MS (top), but distinguishable by SEM-EDS (bottom) on the basis of the silicon and titanium 
For SEM-EDS, Si, Ti, Mn, and Co are above the paper blank (though Mn and Co have small peaks just above the paper background); the remaining elements are below the paper contribution and are thus excluded for discrimination purposes. The two distinguishing elements ( $\mathrm{Si}$ and $\mathrm{Ti}$ ) using SEM-EDS are approximately equal to the paper contribution using LA-ICP-MS.

All three quality controls and three duplicates were correctly associated using LA-ICPMS, resulting in $100 \%$ correct association.

\subsubsection{Intaglio Inks}

LA-ICP-MS was capable of discriminating nearly all, $99.9 \%$, of the intaglio samples; only two pairs were indistinguishable: Intaglio 10 with Intaglio 11 (Ukrainian banknote) and Intaglio 35 with Intaglio 36 (Italian banknote). Both pairs were comparisons between specimens sampled from different areas of the same banknote. Intaglio 35 and Intaglio 36 were the same color (brown), while Intaglio 10 and Intaglio 11 were different colors (red versus gold, respectively). The two false inclusions were also associated using SEMEDS, thus SEM-EDS did not provide complementary information for intaglio inks. Figure 31 shows the spectra of an indistinguishable intaglio pair (Intaglio 10 and 11) as well as three intaglio samples that were distinguished by unusual elements $(\mathrm{Mo}, \mathrm{Sb}, \mathrm{Pb}$, and $\mathrm{Bi}$ ). The seven quality controls and 16 duplicates were correctly associated, resulting in no false exclusions. 


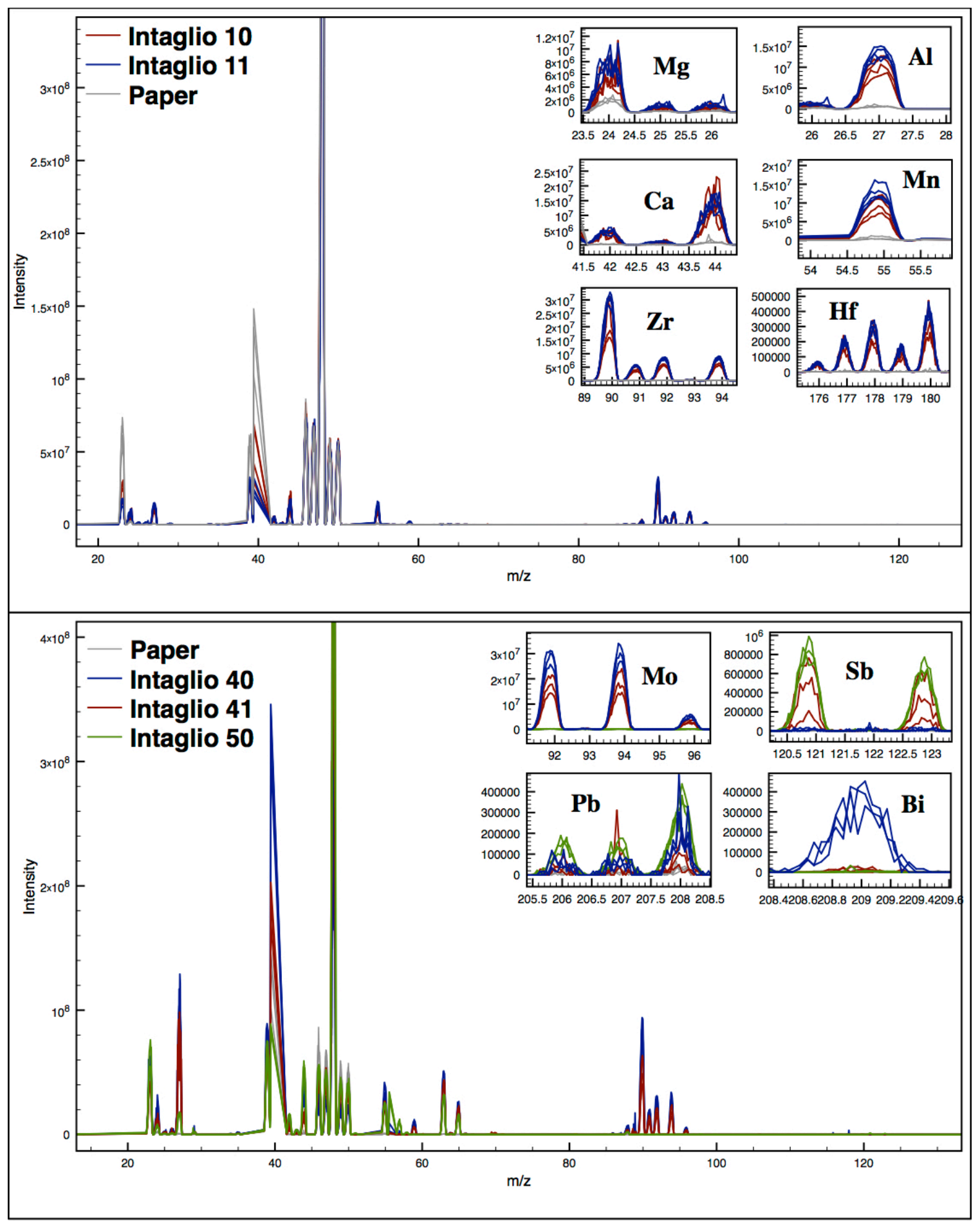

Figure 31 - Pair of intaglio samples that were indistinguishable (top) and three intaglio samples that were distinguishable by unusual elements (bottom) 


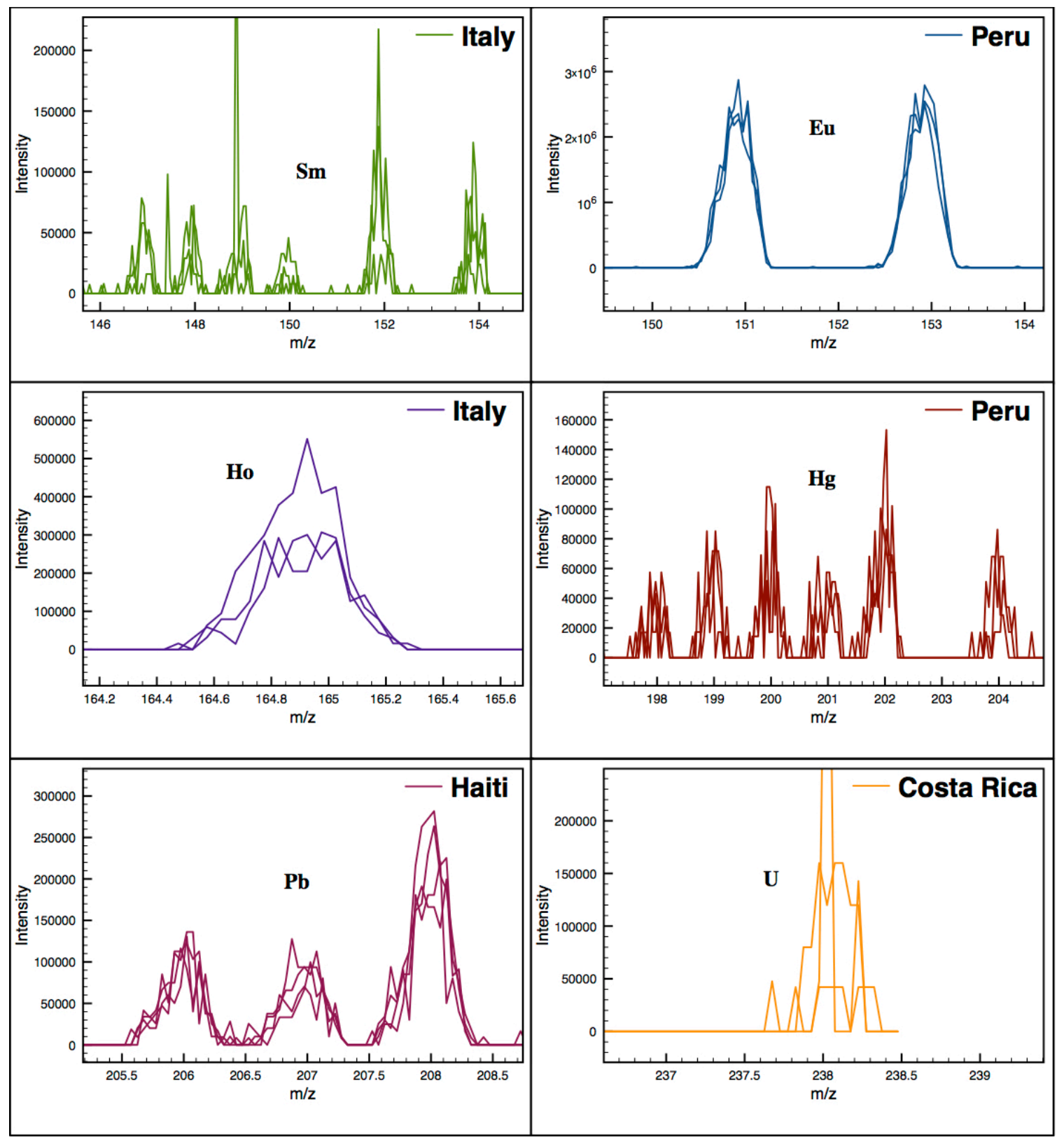

Figure 32 - Examples of unusual elements detected in banknote paper

Paper contribution was irrelevant for the analysis of the intaglio samples by SEM-EDS since the ink layer thickness of the intaglio samples was in the range of $19-45 \mu \mathrm{m}$. However, for LA-ICP-MS, the laser ablates both ink and paper. Thus, only the elements that were above the paper threshold were considered to be present in the ink and included 
for discrimination purposes. Several unusual elements were detected in the banknote paper (Figure 32). These elements may be present as contaminants in the raw materials or may be intentionally added for security purposes. ${ }^{1}$

\subsection{LIBS Analysis of Toners and Inkjets}

Although not originally included as part of the ink project, the discrimination and association potential of Laser Induced Breakdown Spectroscopy (LIBS) was evaluated using a subset of inkjet and toner samples. Offset and intaglio samples were excluded since the original samples were quite small and insufficient ink remained after analysis using several techniques (SEM-EDS, LA-ICP-MS, FTIR, py-GC-MS, DART-MS, and Raman). ${ }^{145} \mathrm{~A}$ total of 45 inkjet and 57 toner samples were analyzed using LIBS.

Optimization of SEM-EDS and LA-ICP-MS parameters involved changing one parameter at a time and selecting the value that provided the best compromise between a high signal-to-noise ratio and a low relative standard deviation (RSD). This optimization approach, referred to as a "one-at-a-time design," is time consuming and does not account for interactions between different parameters. An alternative method involves a Design of Experiments (DoE) approach. When using DoE, all parameters are varied simultaneously in order to identify which parameters significantly affect the response(s) of interest (SNR and RSD). ${ }^{146}$ Moreover, a DoE approach requires fewer experimental runs, significantly reducing the time required for optimization. DoE optimization was

accomplished using the "Definitive Screening Design" option in JMP (version 11). ${ }^{147-148}$ A total of 13 experimental runs were necessary in order to optimize the five LIBS parameters: laser energy, laser frequency, gate delay, stage speed, and argon flow rate. 
The spot size $(100 \mu \mathrm{m})$ and the line length $(2 \mathrm{~mm})$ were held constant; these values were selected based on previous LIBS experiments within the research group. For each parameter, a low value and a high value are specified; the JMP software then adds a middle point. For example, the low value for the laser energy was specified at $80 \%$ and the high value was specified at $100 \%$ (the middle value is thus $90 \%$ ). JMP then creates a table that specifies the value of each parameter for every experimental run.

Figure 33 shows the 13 experimental runs needed to optimize the LIBS parameters for the analysis of inkjets. Run 1 indicates that the laser energy should be set to $100 \%$, the frequency to $7 \mathrm{~Hz}$, the stage speed to $0.45 \mathrm{~mm} / \mathrm{s}$, the gate delay to $0.1 \mu \mathrm{s}$, and the argon flow rate to $1 \mathrm{~L} / \mathrm{min}$; note that the runs are randomized, which is why the run numbers are out of order. The alternating blue and purple pairs have one parameter set to the middle (shown in bold) and the remaining parameters at either extreme. When a parameter for one of the paired runs is set to the high value, the same parameter is set to the low value for the other paired run. For example, the paired runs 11 and 8 have the laser energy set to the midpoint, $90 \%$; all remaining parameters are set to the high value for run 11 and to the low value for run 8 . For the last run, shown in red, all parameters are at the center point. Finally, two additional runs (shown in green) have no parameters at the middle point.

Four replicate measurements were collected for each experimental run using a J200 LIBS system (Applied Spectra, Fremont CA, USA). The SNR and RSD were then calculated for elements of interest using the integrated peak areas; element peaks were integrated using the data analysis software included with the J200 (Aurora, version 1.0013). 


\begin{tabular}{|c|c|c|c|c|c|}
\hline Run Number & Laser Energy & Frequency & Stage Speed & Gate Delay & Ar Gas Flow \\
\hline & $\%$ & $\mathrm{~Hz}$ & $\mathrm{~mm} / \mathrm{s}$ & $\mu s$ & $\mathrm{~L} / \mathrm{min}$ \\
\hline 11 & 90 & 9 & 0.5 & 0.5 & 1 \\
\hline 8 & 90 & 7 & 0.4 & 0.1 & 0.4 \\
\hline 12 & 80 & 8 & 0.5 & 0.1 & 0.4 \\
\hline 13 & 100 & 8 & 0.4 & 0.5 & 1 \\
\hline 1 & 100 & 7 & 0.45 & 0.1 & 1 \\
\hline 10 & 80 & 9 & 0.45 & 0.5 & 0.4 \\
\hline 4 & 80 & 7 & 0.5 & 0.3 & 1 \\
\hline 7 & 100 & 9 & 0.4 & 0.3 & 0.4 \\
\hline 2 & 100 & 9 & 0.5 & 0.1 & 0.7 \\
\hline 5 & 80 & 7 & 0.4 & 0.5 & 0.7 \\
\hline 3 & 80 & 9 & 0.4 & 0.1 & 1 \\
\hline 9 & 100 & 7 & 0.5 & 0.5 & 0.4 \\
\hline 6 & 90 & 8 & 0.45 & 0.3 & 0.7 \\
\hline
\end{tabular}

Figure 33 - Definitive Screening Design to optimize LIBS parameters for inkjet analysis

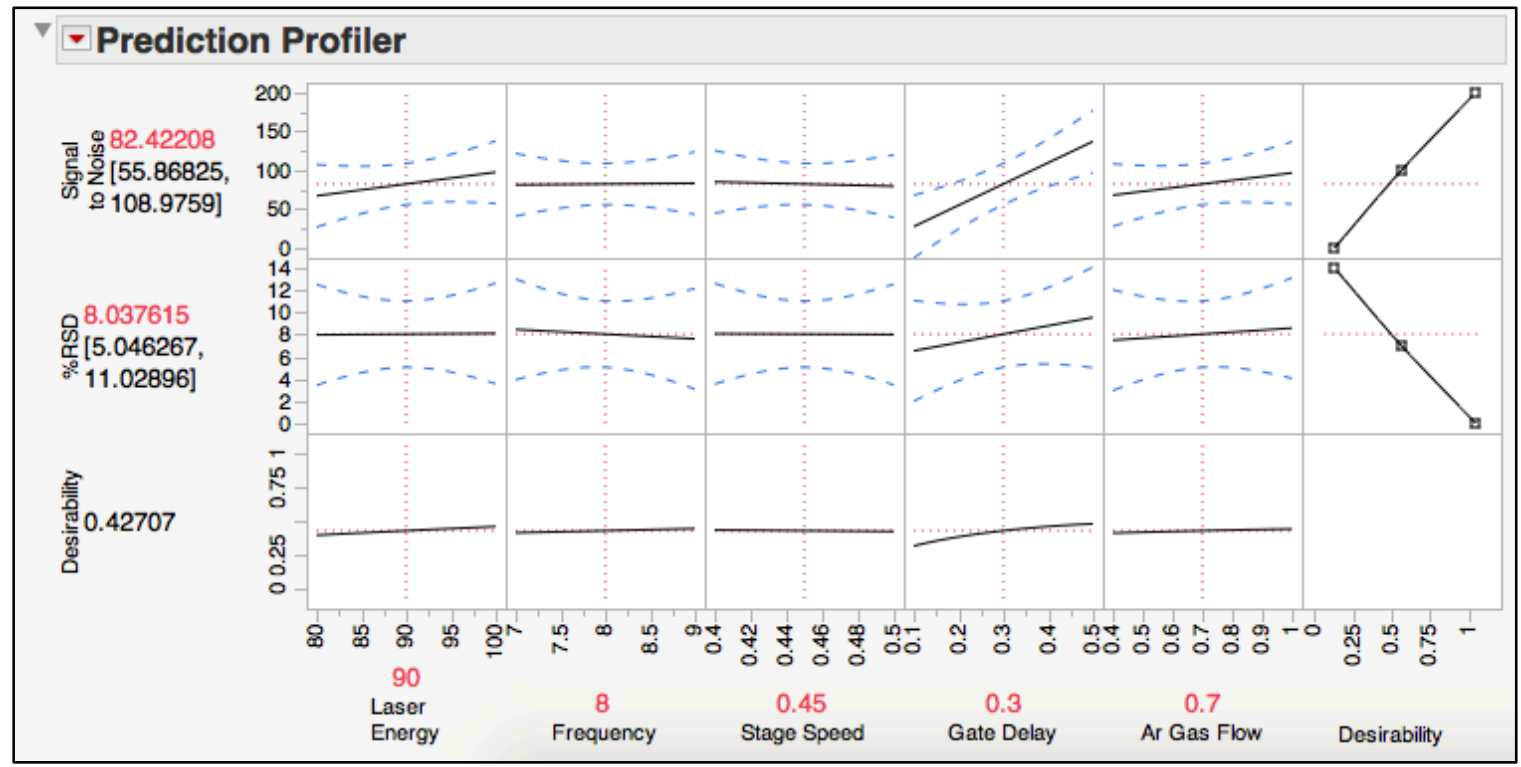

Figure 34 - Prediction Profiler in JMP showing parameter values expected to provide the best compromise between SNR and RSD for sodium 
The "Prediction Profiler" in JMP provides the parameter values expected to result in the best compromise between SNR and RSD. Figure 34 shows a screenshot of the Prediction Profiler for one element, sodium. The model fitting and prediction must be done individually for each element of interest. The analyst then decides on the best overall parameters for all elements of interest. The same optimization procedure was applied to the toner samples. Table 17 shows the optimized parameters for inkjets and toners as well as the elements that were detected in each ink type (SNR $\geq 3)$. A lower laser energy and frequency as well as a faster stage speed were necessary for inkjets in order to minimize the removal of the paper.

Table 17 - Optimized LIBS parameters and elements detected for each ink type

\begin{tabular}{ccc}
\hline & INKJET & TONER \\
\hline Laser Energy $(\%)$ & 90 & 100 \\
Frequency $(\mathbf{H z})$ & 8 & 9 \\
Stage Speed $(\mu \mathrm{m} / \mathbf{s})$ & 0.45 & 0.4 \\
Gate Delay $(\boldsymbol{\mu s})$ & 0.3 & 0.3 \\
Argon Flow $(\mathrm{L} / \mathbf{m i n})$ & 0.7 & 0.4 \\
Spot Size $(\mu \mathrm{m})$ & 100 & 100 \\
Line Length $(\mathbf{m m})$ & 2 & 2 \\
Elements Detected & $\mathrm{Cu}, \mathrm{Li}, \mathrm{K}, \mathrm{Mg}, \mathrm{Na}$ & $\mathrm{Al}, \mathrm{Ba}, \mathrm{Cr}, \mathrm{Cu}, \mathrm{Fe}, \mathrm{K}, \mathrm{Mg}, \mathrm{Na}, \mathrm{Si}, \mathrm{Sr}, \mathrm{Ti}$ \\
\hline
\end{tabular}

\subsubsection{Inkjet Inks}

All inkjet pairs were compared using spectral overlay, which resulted in a discrimination of $93.8 \%$ (61 indistinguishable pairs out of 990 total pairs). Thus, LIBS performed better than SEM-EDS (78.4\% discrimination), but not as well as LA-ICP-MS (99.6\% discrimination); this is unsurprising since the detection limit for LIBS typically falls between the two techniques. Of the 61 false inclusions, 15 were comparisons between 
different brands and different colors (mostly magenta with black or yellow inks). A total of 5 elements were detected in the inkjet set: $\mathrm{Cu}, \mathrm{Li}, \mathrm{K}, \mathrm{Mg}$, and $\mathrm{Na}$. Although calcium was also detected, its paper blank emission signal was quite high. It was not feasible to determine whether part of the calcium signal could be attributed to the ink, thus calcium was excluded from the element menu.

To evaluate the correct association rate, a total of 8 quality controls $(8 \times 7 \div 2=28$ pairwise comparisons) and 10 duplicates (10 pairwise comparisons) were analyzed. As described earlier, a quality control is a single sample that is analyzed multiple times during an experimental run and/or over several days; the purpose of a quality control is to assess the intra-day and/or inter-day reproducibility. A duplicate is a sample that is prepared and analyzed a second time; a set of duplicate samples can be analyzed in order to assess the correct association rate. It should be noted, however, that quality controls could be treated as duplicates that have simply been analyzed more than twice.

Unfortunately, LIBS exhibited poor reproducibility, which resulted in a high false exclusion rate for the duplicates and controls. The spectra were subsequently normalized in an effort to improve the reproducibility. Energy normalization was used, which assumes that the total energy of each spectrum is constant across shots. ${ }^{149}$ Figure 35 shows a comparison of the raw versus the normalized spectra for a pair of quality controls and a pair of duplicates. Prior to normalization, the duplicates (Inkjet 38) were falsely excluded by $\mathrm{Na}, \mathrm{Li}$, and $\mathrm{K}$. However, after normalization, the pair was correctly associated. Prior to normalization, the quality controls were falsely excluded by $\mathrm{Cu}, \mathrm{Na}$, $\mathrm{Li}$, and $\mathrm{K}$. After normalization, the pair was still excluded by Li (but the signals were much closer). Overall, even after normalization, the correct association was fairly poor: 2 
quality control pairs and 6 duplicate pairs were falsely excluded, resulting in a correct association of $78.9 \%$ (30 out of 38 pairs).
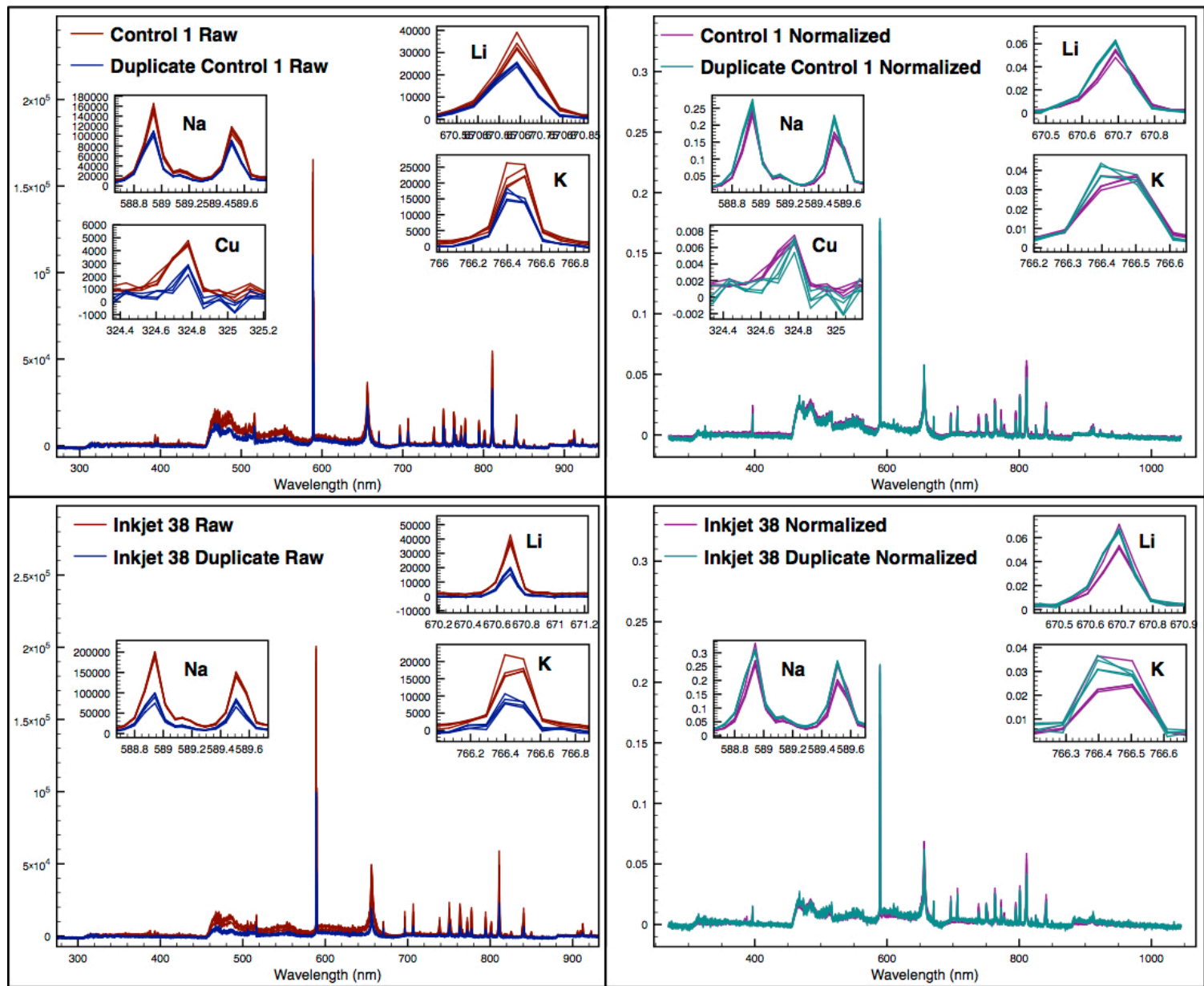

Figure 35 - Raw (left) versus normalized (right) spectra for a pair of quality controls (top) and a pair of duplicates (bottom)

In order to improve the correct association results, an alternative approach to data analysis was investigated. The peak areas for the elements of interest were integrated as described for LIBS optimization. The following element ratios (along with the emission wavelength) were then selected based on their RSDs and discrimination capabilities. 


$$
\frac{N a 588}{L i 670} \frac{L i 670}{K 766} \frac{N a 588}{K 766} \frac{L i 670}{M g 279} \frac{C u 327}{M g 280} \frac{K 770}{M g 279} \frac{M g 279}{C u 324} \frac{N a 588}{M g 279} \frac{K 766}{C u 324}
$$

A match criterion, similar to the one described in the standard methodology for the analysis of glass using LA-ICP-MS (ASTM E2927), was used for the LIBS pairwise comparisons. ASTM E2927 suggests a modified \pm 4 standard deviations for the comparison between two samples. ${ }^{115}$ First, the average and standard deviation for each element (or in the case of LIBS, element ratio) of sample 1 are calculated. If the calculated standard deviation is less than $3 \%$ of the mean, then $3 \%$ of the mean is used instead of the standard deviation. The comparison interval for sample 1 is then defined as the average \pm 4 times the standard deviation (or \pm 4 times $3 \%$ of the average, whichever is greatest). The average of sample 2 is then calculated and compared to the sample 1 comparison interval. This procedure is done for all elements (or ratios) measured. If the average of sample 2 lies within the comparison interval of sample 1 for all elements/ratios, then the two samples are indistinguishable. Alternatively, if the average of sample 2 lies outside the sample 1 comparison interval for one or more element(s)/ratio(s), then the two samples are distinguishable. Since LA-ICP-MS exhibits high precision, a $3 \%$ minimum RSD was necessary to avoid a high false exclusion rate. In the case of LIBS, reproducibility was quite poor and a higher minimum was necessary to avoid a high false exclusion rate. The new minimum for each element ratio was set to the RSD calculated using the 8 controls; the RSDs ranged from $4.2 \%$ to as high as $25.9 \%$, depending on the element ratio. This approach is similar to what the Bundeskriminalamt (BKA) has reported for LA-ICP-MS glass analysis. ${ }^{37}$ The BKA established a "Fixed 
Relative Standard Deviation" (FRSD) by calculating the RSD for each element using data collected from a control sample that was analyzed 90 times. However, unlike the ASTM match criterion, the BKA match criterion does not use the standard deviation (SD) of sample 1; in the latter case, the comparison interval uses an RSD that is "fixed" to the value that was calculated from the control data. In the current methodology, the comparison interval is given by the average $\pm 4 \times \mathrm{SD}$ (or $\pm 4 \times \mathrm{RSD} \%$ of the average, whichever is greatest).

Figure 36 shows the control charts for the 8 quality controls using the 9 element ratios. The green solid line is the overall average, the dashed grey line is the average $\pm 5 \%$, and the dotted grey line is the average $\pm 20 \%$. As can be seen for most ratios, many controls deviate more than 5\% from the overall average and a few deviate more than $20 \%$. These charts illustrate the poor reproducibility of LIBS and the necessity of establishing a high minimum RSD for the pairwise comparisons. The most reproducible ratio was Na588/Li670 (RSD 4.2\%) followed by Li670/K766 and Na588/K766 (both with an RSD of 7.3\%). These ratios likely performed best since these 3 elements ( $\mathrm{Li}, \mathrm{Na}$, and $\mathrm{K}$ ) are excellent emitters and had an intense emission peak (see top left panel of Figure 35). On the other hand, ratios involving Mg had an RSD between 22 and 26\%, likely because $\mathrm{Mg}$ was not detected in the control sample. However, these ratios were included because $\mathrm{Mg}$ is present in other inkjet samples. It should be noted that these RSDs were calculated for a small set of controls (8 controls analyzed over 3 days); a larger set of controls analyzed over a longer period of time is necessary to obtain a more accurate measure of the interday variation. 

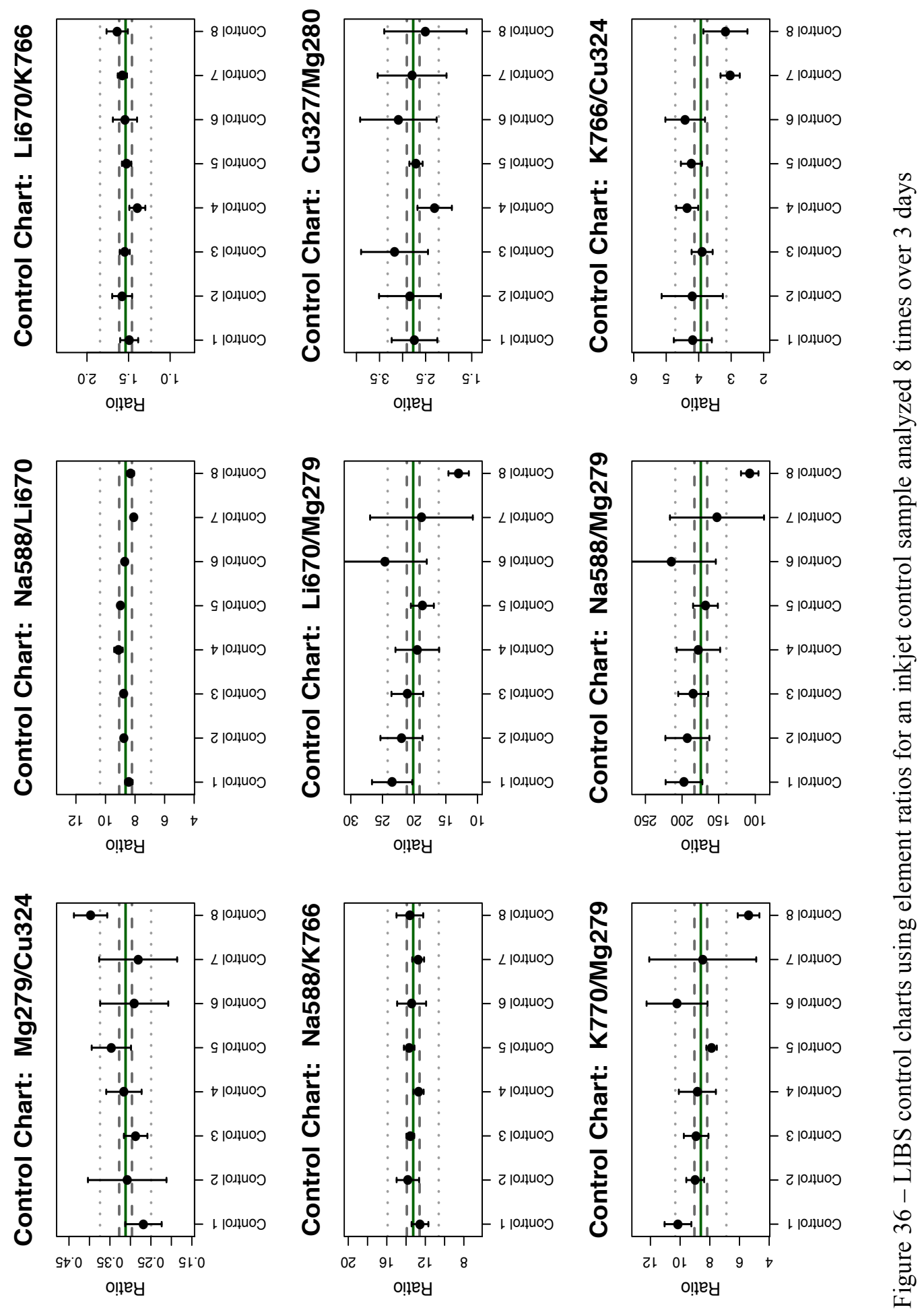
Table 18 shows the overall and individual color discrimination for inkjet inks using the match criterion described above. The discrimination for same-color comparison pairs is also included. Note that the number of pairwise comparisons is given by $n \times n-1$ because the match criterion is asymmetrical (i.e., the results depend on which sample is treated as sample 1 and which is treated as sample 2). Thus each comparison pair was analyzed twice so that each sample in the pair was treated as sample 1 and sample 2.

Table 18 - Discrimination (bolded), correct association (bolded), and false inclusion/exclusion rate for all LIBS inkjet comparisons as well as for each individual color. Values in parentheses indicate either the number of pairwise comparisons or the fraction of discriminated/associated pairs.

\begin{tabular}{cccccc}
\hline & OVERALL & BLACK & CYAN & MAGENTA & YELLOW \\
\hline Number of & 45 & 17 & 10 & 10 & 8 \\
Samples & $(1980)$ & $(272)$ & $(90)$ & $(90)$ & $(56)$ \\
Number of & 10 & 2 & 3 & 4 & 1 \\
Duplicates & $(10)$ & $(2)$ & $(3)$ & $(4)$ & $(1)$ \\
Number of & 8 & 0 & 8 & 0 & 0 \\
Controls & $(28)$ & $(0)$ & $(28)$ & $(0)$ & $(0)$ \\
Discrimination & $\mathbf{9 5 . 9 \%}$ & $\mathbf{8 6 . 8 \%}$ & $\mathbf{9 5 . 6 \%}$ & $\mathbf{9 7 . 8 \%}$ & $\mathbf{8 3 . 9 \%}$ \\
Correct & $(1899 / 1980)$ & $(236 / 272)$ & $(86 / 90)$ & $(88 / 90)$ & $(47 / 56)$ \\
Association & $\mathbf{9 4 . 7 \%}$ & $\mathbf{5 0 . 0 \%}$ & $\mathbf{9 6 . 8 \%}$ & $\mathbf{1 0 0 \%}$ & $\mathbf{1 0 0 \%}$ \\
False Inclusion & $(36 / 38)$ & $(1 / 2)$ & $(30 / 31)$ & $(4 / 4)$ & $(1 / 1)$ \\
& $(81 / 1980)$ & $13.2 \%$ & $4.4 \%$ & $2.2 \%$ & $16.1 \%$ \\
False Exclusion & $5.3 \%$ & $(36 / 272)$ & $(4 / 90)$ & $(2 / 90)$ & $(9 / 56)$ \\
& $(2 / 38)$ & $(1 / 2)$ & $(1 / 31)$ & $(0 / 4)$ & $(0 / 1)$ \\
\hline
\end{tabular}

LIBS provided an overall discrimination of $95.9 \%$ and an overall (including duplicates and controls) correct association of $94.7 \%$ using the match criterion previously described. All controls were correctly associated and 8 out of 10 duplicates were correctly associated. The match criterion provided better results than the more subjective spectral overlay comparisons, even after energy normalization. LIBS also provided excellent 
discrimination and association (>95\%) of cyan and magenta inks. The discrimination and association of black inkjets was more limited ( $86.8 \%$ and $50 \%$, respectively). However, LIBS is more suitable for the analysis of inkjets than SEM-EDS, which resulted in extremely poor discrimination for black inkjets (49.2\%). Additionally, the correct association for black inks was estimated using only 2 duplicates; a much larger set is necessary to more accurately estimate the false exclusion rate. Finally, LIBS performed poorest for the yellow inkjets, resulting in relatively low discrimination of $83.9 \%$. This low discrimination may be because most yellow inkjets contained only two detectable elements $(\mathrm{Na}$ and/or $\mathrm{K})$ at similar concentrations. Overall, LIBS provided improved discrimination for black, cyan, and magenta inkjets and similar discrimination for yellow inkjets. Thus, LIBS offers an attractive alternative to SEM-EDS: higher discrimination, faster analysis $(\sim 1$ minute versus 20 minutes per replicate), and less expensive instrumentation.

\subsubsection{Toner Inks}

Spectral overlay of toners resulted in good discrimination (97.9\%). A total of 11 elements were detected in the toner set: $\mathrm{Al}, \mathrm{Ba}, \mathrm{Cr}, \mathrm{Cu}, \mathrm{Fe}, \mathrm{K}, \mathrm{Mg}, \mathrm{Na}, \mathrm{Si}, \mathrm{Sr}$, and Ti. Calcium was detected as well but excluded for discrimination purposes since it was present in high amounts in the blank paper. Of the 33 false inclusions, only 4 were comparisons between different brands (Hewlett Packard versus Canon) and different colors (magenta versus black or yellow). Unfortunately, as was the case for inkjets, spectral overlay provided poor correct association (21.7\%). Only 1 out of 10 duplicates and 9 out of 36 controls were correctly associated. To improve the correct association rate, the match criterion 
described earlier was used for pairwise comparisons. The following 21 ratios were selected for the comparisons.

$\frac{N a 588}{K 766} \frac{M g 279}{B a 455} \frac{S r 408}{B a 455} \frac{C u 324}{F e 273} \frac{T i 336}{B a 455} \frac{S i 288}{A l 396} \frac{A l 396}{K 766} \frac{M g 279}{A l 396} \frac{B a 455}{C r 283} \frac{C u 324}{B a 455} \frac{B a 455}{F e 273}$
$\frac{K 766}{B a 455} \frac{\text { Si288 }}{\text { Ba455 }} \frac{F e 273}{C r 283} \frac{K 766}{C r 283} \frac{\text { Na588 }}{\text { Cr283 }} \frac{K 766}{C u 324} \frac{K 766}{F e 273} \frac{\text { Sr } 408}{F e 273} \frac{\text { Sr } 408}{K 766} \frac{\text { Ti336 }}{K 766}$

As was the case for inkjets, the overall RSD of each ratio for the 9 control samples was selected as the minimum RSD for the pairwise comparisons. For toners, the RSDs ranged from $15.5 \%$ to a high $37.3 \%$ (ratios with RSD $>40 \%$ were excluded for discrimination). Figure 37 shows the control charts for several selected ratios. None of the ratios, including the ones not shown in the figure, fell within the average $\pm 5 \%$ for all 9 controls. Additionally, nearly all ratios had at least one control sample that fell outside the average $\pm 20 \%$. Finally, the standard deviation was quite large for most controls, as indicated by the large error bars in the control charts. The control charts highlight the high intra-day and inter-day variation for LIBS analysis. The ratio with the best RSD was Si288/Al396 (15.5\% RSD); both elements were present at high concentration in the control sample. On the other hand, the ratio with the poorest RSD was Mg279/Ba455 (37.3\% RSD); Ba was not detected in the control, but is an important element for other toner samples. 

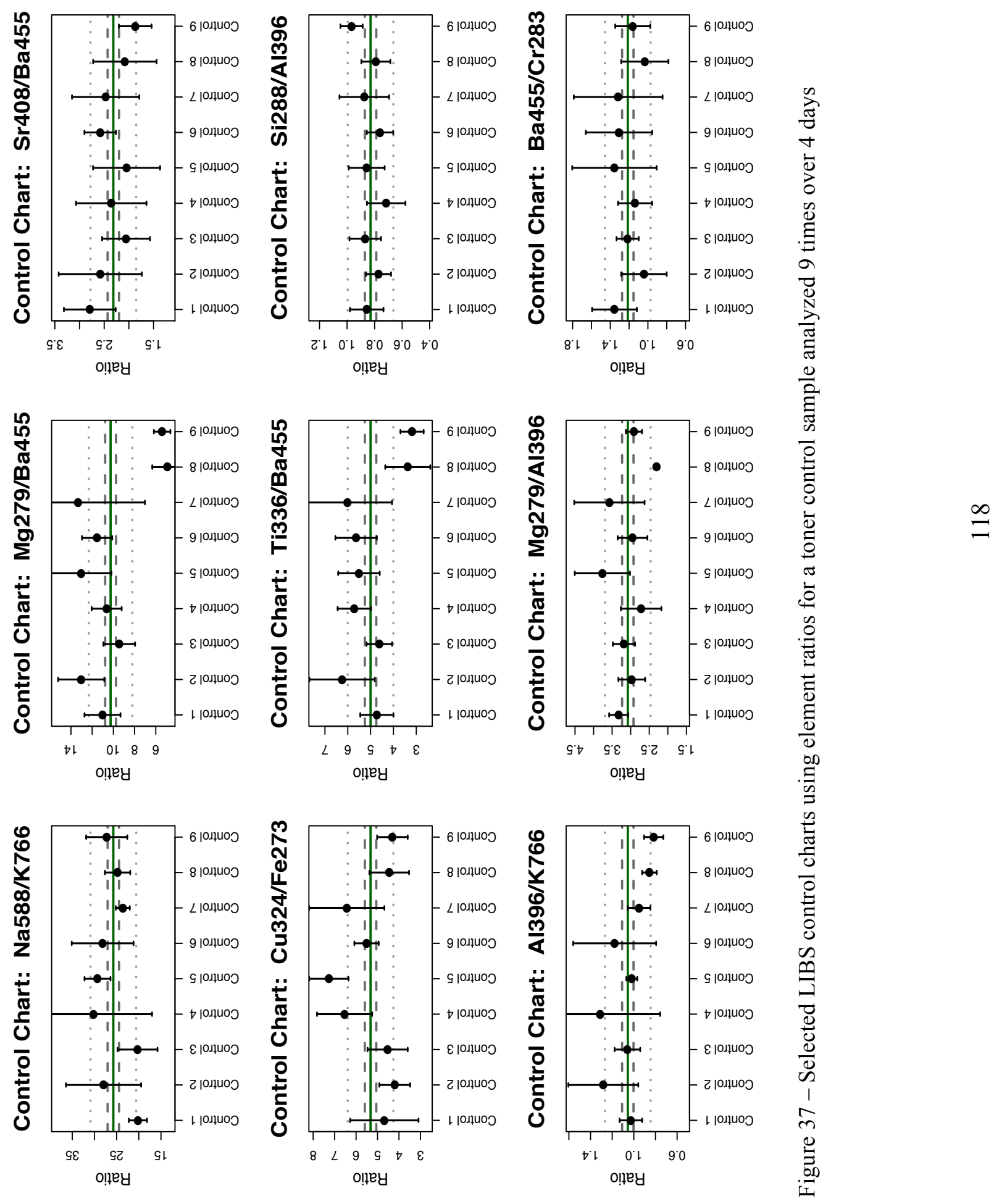
Table 19 shows the overall and individual color discrimination for toner inks using the modified \pm 4 SD match criterion. LIBS provided an overall discrimination of $96.9 \%$ and an overall (including duplicates and controls) correct association of $89.1 \%$ using the match criterion. For the controls, 33 out of 36 pairs were correctly associated and for the duplicates, 8 out of 10 pairs were correctly associated. Compared to spectral overlay, using a match criterion significantly improved the correct association rate.

Table 19 - Discrimination (bolded), correct association (bolded), and false inclusion/exclusion rate for all LIBS toner comparisons as well as for each individual color. Values in parentheses indicate either the number of pairwise comparisons or the fraction of discriminated/associated pairs.

\begin{tabular}{cccccc}
\hline & OVERALL & BLACK & CYAN & MAGENTA & YELLOW \\
\hline Number of & 57 & 23 & 11 & 11 & 12 \\
Samples & $(3192)$ & $(506)$ & $(110)$ & $(110)$ & $(132)$ \\
Number of & 10 & 6 & 2 & 1 & 1 \\
Duplicates & $(10)$ & $(6)$ & $(2)$ & $(1)$ & $(1)$ \\
Number of & 9 & 9 & 0 & 0 & 0 \\
Controls & $(36)$ & $(36)$ & $(0)$ & $(0)$ & $(0)$ \\
Discrimination & $\mathbf{9 6 . 9 \%}$ & $\mathbf{9 5 . 8 \%}$ & $\mathbf{9 8 . 2 \%}$ & $\mathbf{9 8 . 2 \%}$ & $\mathbf{9 5 . 5 \%}$ \\
Correct & $(3094 / 3192)$ & $(485 / 506)$ & $(108 / 110)$ & $(178 / 110)$ & $(126 / 132)$ \\
Association & $\mathbf{8 9 . 1 \%}$ & $\mathbf{8 8 . 1 \%}$ & $\mathbf{1 0 0 \%}$ & $\mathbf{1 0 0 \%}$ & $\mathbf{1 0 0 \%}$ \\
False Inclusion & $(41 / 46)$ & $(37 / 42)$ & $(2 / 2)$ & $(1 / 1)$ & $(1 / 1)$ \\
& $(98 / 3 \%$ & $4.2 \%$ & $1.8 \%$ & $1.8 \%$ & $4.5 \%$ \\
False Exclusion & $10.9 \%$ & $(21 / 506)$ & $(2 / 110)$ & $(2 / 110)$ & $(6 / 132)$ \\
& $(5 / 46)$ & $(5 / 42)$ & $(0 / 2)$ & 0 & 0 \\
\hline
\end{tabular}

LIBS also provided excellent discrimination $(>95 \%)$ and good correct association $(>$ $88 \%$ ) for all individual toner ink colors. Thus, LIBS provides an improvement over SEMEDS and similar results to LA-ICP-MS, making LIBS a viable alternative to both SEMEDS and LA-ICP-MS for the analysis of toner inks of the same color. 


\subsection{FIACS Database}

Part of the ink project involved the development of the Forensic Ink Analysis and Comparison System (FIACS), a searchable database that allows the analyst to compare a questioned ink sample to a reference collection in order to narrow down the possibilities of the questioned ink's origin. ${ }^{145}$ The FIACS database utilizes two classification tools: Partial Least Squares Discriminant Analysis (PLSDA) and K-Nearest Neighbor (KNN). The algorithms were created in house using Matlab and a user-friendly Graphical User Interface (GUI) is provided. Figure 38 shows an example of the FIACS PLSDA and KNN output.

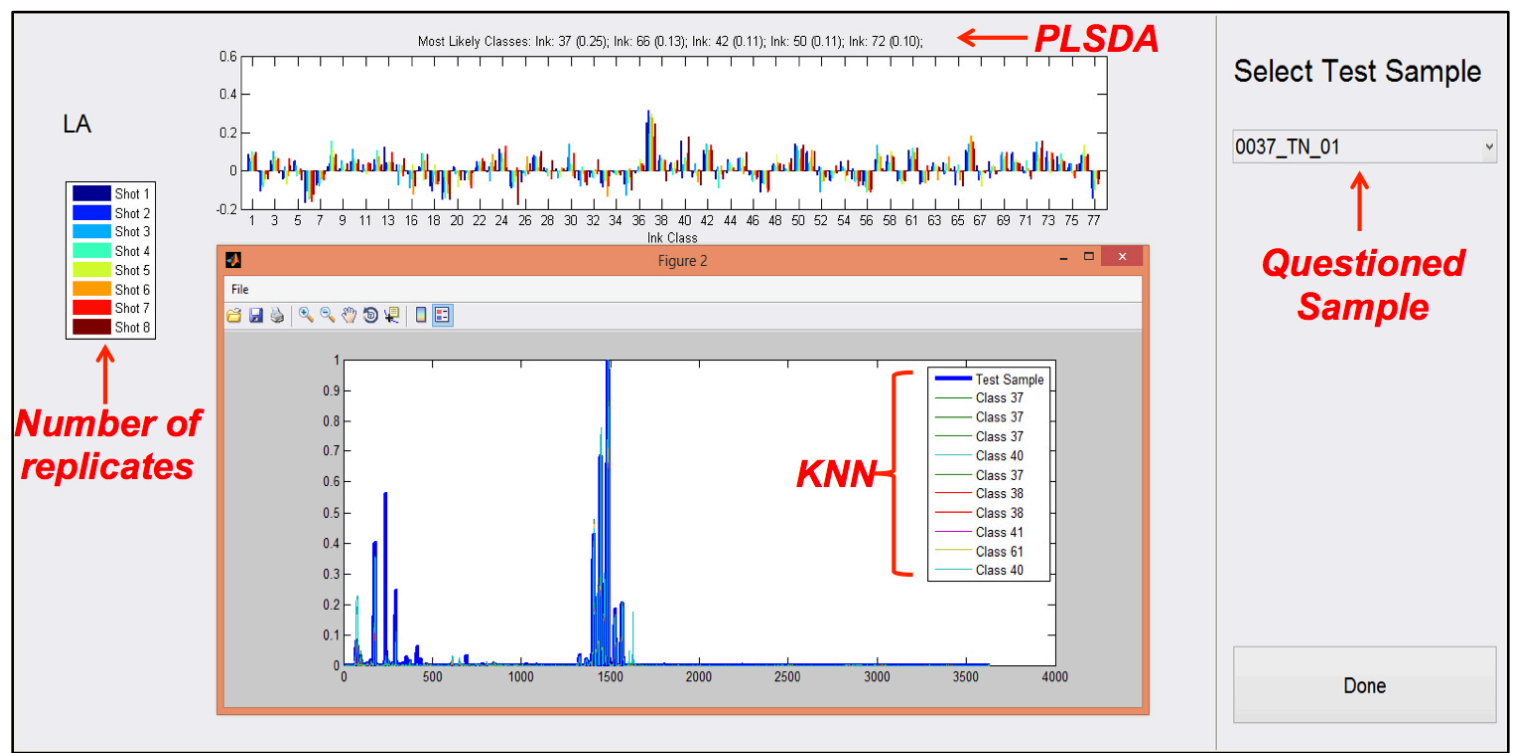

Figure 38 - Example of FIACS PLSDA and KNN output

In PLSDA, Partial Least Squares Regression (PLSR) is used to create linear combinations of predictor variables rather than the original variables (i.e., elements). Variables that show a high correlation with the response variables (i.e., intensity) are given extra weight. ${ }^{146}$ By default, the number of PLSDA components for the FIACS 
database is set to the number of unique samples in the reference collection plus one. For example, for toners ( $\mathrm{N}=76$ samples), the number of PLSDA components would be equal to $76+1=77$. After the comparison of the questioned sample to the reference database, the FIACS database provides the 5 reference samples that are closest to the questioned sample. Figure 39 shows a close up of the FIACS PLSDA output (from Figure 38) using a duplicate sample, Toner 37 , as the questioned sample. The first of the 5 inks listed on top of the graph is the sample that is most similar to the questioned sample, as indicated by the highest similarity score (values in parentheses). The most similar sample in the reference database is Toner 37 (circled in black), with a similarity score of 0.25 ; this is unsurprising since the questioned sample is a duplicate of Toner 37.

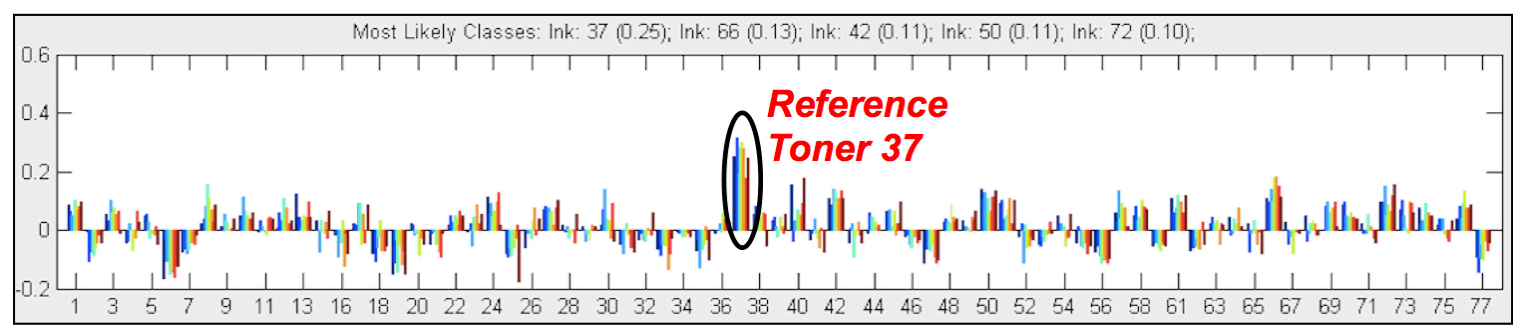

Figure 39 - Close up of FIACS PLSDA output for duplicate Toner 37

The next most similar samples are: Toner $66(0.13)$, Toner $42(0.11)$, Toner $50(0.11)$, and Toner $72(0.10)$. The colored bars in the graph indicate the 8 replicates for each toner sample in the reference database.

In KNN, samples are compared using a distance measure, usually the Euclidean distance. For the FIACS database, the $\min / \max [0,1]$ normalized Euclidean distance is used to compare spectra. That is, all spectra are normalized so that the lowest intensity is set to 0 and the highest intensity is set to 1 . This normalization helps reduce within-source 
variation because of changes in experimental conditions. After normalization, the Euclidean distance is calculated as follows:

$$
d=\sqrt{\left(x_{1}-y_{1}\right)^{2}+\left(x_{2}-y_{2}\right)^{2}+\cdots+\left(x_{n}-y_{n}\right)^{2}}
$$

In the formula above, $x$ represents the intensity for each data point of sample 1 and $y$ represents the intensity for each data point of sample 2. Thus, the Euclidean distance takes the difference between the y-axis values (intensity) of two samples for every $\mathrm{x}$-axis data point (e.g., m/z, energy, wavelength). Then the squared differences are summed and the square root of the sum is taken. ${ }^{146}$ The FIACS database lists the 10 reference spectra that are closest (smallest Euclidean distance) to the questioned sample being compared to the reference database.

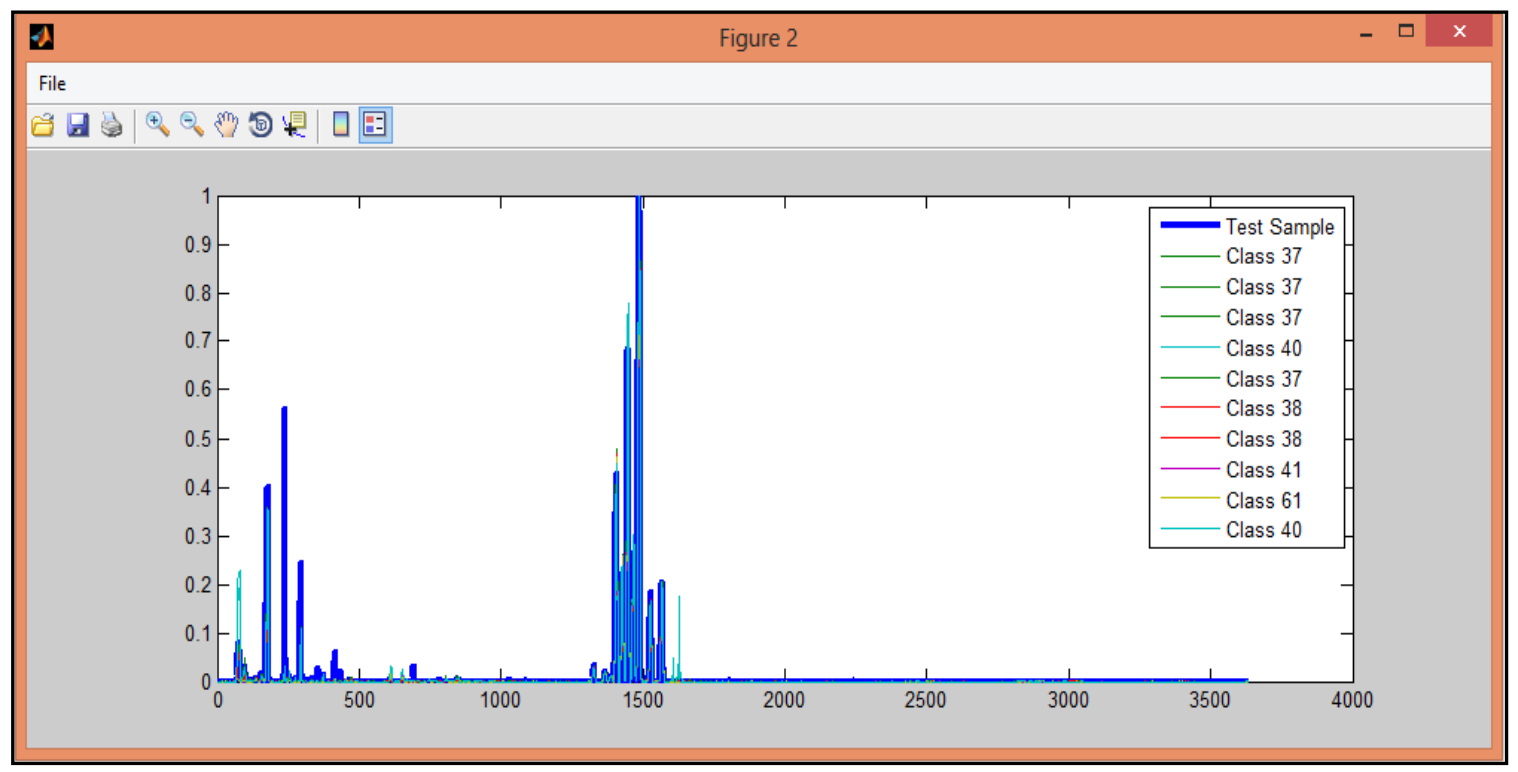

Figure 40 - Close up of FIACS KNN output for duplicate Toner 37

Figure 40 shows a close up of the KNN output (from Figure 38) for the duplicate Toner 37. The KNN window allows the user to compare one replicate of the questioned sample 
(shown in blue) to the top 10 closest spectra in the reference database. Reference Toner 37 appears on the top 3 hits. The top 10 closest spectra is given for each individual replicate of the questioned sample, thus the top 10 spectra may differ between different replicate measurements for the questioned sample.

In order to test the performance of the FIACS database, the duplicate samples for each type of ink were treated as questioned samples. The correct classification rate was then calculated for the each classification tool, PLSDA and KNN. For PLSDA, if the correct reference ink appeared as one of the top 5 inks, then the reference and duplicate ink were correctly associated. For KNN, if the correct reference ink appeared at least once in the top 10 spectra for every replicate of the questioned (duplicate) sample, then the reference and duplicate ink were correctly associated. The results for each type of ink using SEMEDS and LA-ICP-MS data are presented below. LIBS was excluded from this study since only a fairly small subset of inkjets and toners were analyzed.

\subsubsection{Inkjet Inks}

The correct association results for inkjets using SEM-EDS and LA-ICP-MS are shown in Table 20. LA-ICP-MS resulted in 100\% correct association for both PLSDA and KNN; however, it should be noted that only one duplicate was available for the test set. SEMEDS performed poorly with a low $33.3 \%$ correct association for PLSDA and $50 \%$ correct association for KNN. These results are unsurprising since SEM-EDS showed limited utility for the analysis of inkjet inks. Since many inkjet samples were indistinguishable, several reference samples were confused with the questioned (duplicate) sample, yielding incorrect "matches." For the falsely excluded pairs using KNN, the correct reference ink 
appeared in the top 10 spectra for at least 1 out of the 3 SEM-EDS replicate measurements. However, based on the definition of a correct association given above, these pairs were falsely excluded. Still, since the user is able to visually inspect the spectra, it is ultimately the analyst's responsibility to decide whether a questioned sample is indistinguishable from a similar reference sample. Moreover, when combining the results of PLSDA and KNN, 4 out of the 6 pairs were correctly associated (66.7\%). Thus, using both classification tools may improve the results.

Table 20 - PLSDA and KNN correct association for inkjet inks using SEM-EDS and LA-ICPMS data

\begin{tabular}{|c|c|c|c|c|}
\hline & SEM-EDS & LA-ICP-MS & SEM-EDS & LA-ICP-MS \\
\hline & \multicolumn{2}{|c|}{ PLSDA } & \multicolumn{2}{|c|}{ KNN } \\
\hline Number of Duplicates & 6 & 1 & 6 & 1 \\
\hline Correct Association & $\begin{array}{c}\mathbf{3 3 . 3 \%} \\
(2 / 6)\end{array}$ & $\begin{array}{c}\mathbf{1 0 0 \%} \\
(1 / 1)\end{array}$ & $\begin{array}{c}50.0 \% \\
(3 / 6)\end{array}$ & $\begin{array}{c}\mathbf{1 0 0 \%} \\
(1 / 1)\end{array}$ \\
\hline False Exclusion & $\begin{array}{c}66.7 \% \\
(4 / 6)\end{array}$ & $\begin{array}{c}0 \\
(1 / 1)\end{array}$ & $\begin{array}{c}50.0 \% \\
(3 / 6) \\
\end{array}$ & $\begin{array}{c}0 \\
(1 / 1) \\
\end{array}$ \\
\hline
\end{tabular}

It is worth noting that the larger the reference database and the more replicate measurements collected per sample, the better the performance of the PLSDA and KNN algorithms. The FIACS database can be continuously expanded as more ink samples are collected and analyzed. Additionally, a larger test set would be beneficial in order to truly evaluate the correct association rates.

\subsubsection{Toner Inks}

Table 21 shows the PSLDA and KNN correct association for toner inks. Using LA-ICPMS, there were no false exclusions with KNN and only two false exclusions with 
PLSDA. For one of the false exclusions (Toner 56), the top hit was a sample (Toner 54) that was indistinguishable with the reference sample, which is likely the reason that Toner 56 was confused with Toner 54. SEM-EDS performed better for toners compared to inkjets: $72 \%$ and $60 \%$ correct association using PLSDA and KNN classification, respectively. For KNN classification, the correct reference ink appeared in the top 10 closest spectra for at least one replicate of all 25 duplicate samples.

Table 21 - PLSDA and KNN correct association for toner inks using SEM-EDS and LA-ICP-MS data

\begin{tabular}{|c|cc|cc} 
& \multicolumn{2}{|c|}{ SEM-EDS } & LA-ICP-MS & \multicolumn{2}{c}{ SEM-EDS } & LA-ICP-MS \\
\cline { 2 - 5 } & \multicolumn{2}{c|}{ PLSDA } & \multicolumn{2}{c}{ KNN } \\
\hline \multirow{2}{*}{ Number of Duplicates } & 25 & 20 & 25 & 20 \\
Correct Association & $\mathbf{7 2 . 0} \%$ & $\mathbf{9 0 . 0} \%$ & $\mathbf{6 0 . 0} \%$ & $\mathbf{1 0 0 \%}$ \\
& $(18 / 25)$ & $(18 / 20)$ & $(15 / 25)$ & $(20 / 20)$ \\
False Exclusion & $28.0 \%$ & $10.0 \%$ & $40.0 \%$ & 0 \\
& $(7 / 25)$ & $(2 / 20)$ & $(10 / 25)$ & $(0 / 120)$ \\
\hline
\end{tabular}

When combining PLSDA and KNN, a correct association of $76 \%$ is achieved, which is relatively low considering the good discrimination (97.1\%) that SEM-EDS provided for toners. It should be noted that only 3 replicate measurements were collected for SEMEDS and, as mentioned in the previous section, a greater number of replicates would improve the performance of the algorithms.

\subsubsection{Offset Inks}

The offset inks exhibited the best results: both SEM-EDS and LA-ICP-MS resulted in a high $(>94 \%)$ correct association using either PLSDA or KNN classification (Table 22). 
For SEM-EDS, the KNN falsely included pair showed the correct reference ink in the top 10 spectra for 2 out of the 3 replicate measurements of the questioned ink.

Table 22 - PLSDA and KNN correct association for offset inks using SEM-EDS and LA-ICPMS data

\begin{tabular}{c|cc|cc}
\hline \multirow{2}{*}{ Number of Duplicates } & SEM-EDS & LA-ICP-MS & SEM-EDS & LA-ICP-MS \\
\cline { 2 - 5 } & \multicolumn{2}{|c}{ PLSDA } & \multicolumn{2}{c}{ KNN } \\
Correct Association & 20 & 17 & 20 & 17 \\
& $\mathbf{9 5 . 0} \%$ & $\mathbf{9 4 . 1} \%$ & $\mathbf{9 5 . 0 \%}$ & $\mathbf{9 4 . 1 \%}$ \\
False Exclusion & $(19 / 20)$ & $(16 / 17)$ & $(19 / 20)$ & $(16 / 17)$ \\
& $5.0 \%$ & $5.9 \%$ & $5.0 \%$ & $5.9 \%$ \\
& $(1 / 20)$ & $(1 / 17)$ & $(1 / 20)$ & $(1 / 17)$ \\
\hline
\end{tabular}

Combining the two classification tools did not improve the correct association rate because both PLSDA and KNN falsely excluded the same duplicate pair (Offset 67). The high correct association for SEM-EDS was surprising since the technique performed poorly for the offset ink samples. The thin ink film led to a large paper contribution. When performing spectral overlay comparisons, only the ink signals that were above the paper signal were included for discrimination. However, the raw spectra, which are a mixture of the ink and paper signal, were included in the reference database. Thus, it is possible that the correct association results for the FIACS database are unrealistically high since both elements present in the ink and paper are used for association. It would be beneficial to investigate methods of de-convoluting the ink and paper signal.

\subsubsection{Intaglio Inks}

Like inkjets, few (2-6) duplicates were available to serve as the intaglio test set (Table 23). PLSDA classification performed poorly for SEM-EDS (33.3\% correct association) 
but well for LA-ICP-MS (100\%). The reverse was true for KNN: SEM-EDS performed well (100\%) but LA-ICP-MS performed poorly (50\%). For the KNN falsely included pair using LA-ICP-MS, 9 of the 12 duplicate replicates showed the correct reference sample in the top 10 closest spectra.

Table 23 - PLSDA and KNN correct association for intaglio inks using SEM-EDS and LA-ICPMS data

\begin{tabular}{|c|c|c|c|c|}
\hline & $\overline{\text { SEM-EDS }}$ & $\overline{\text { LA-ICP-MS }}$ & $\overline{\text { SEM-EDS }}$ & $\overline{\text { LA-ICP-MS }}$ \\
\hline & \multicolumn{2}{|c|}{ PLSDA } & \multicolumn{2}{|c|}{ KNN } \\
\hline Number of Duplicates & 6 & 2 & 6 & 2 \\
\hline Correct Association & $\begin{array}{c}\mathbf{3 3 . 3 \%} \mathbf{0} \\
(2 / 6)\end{array}$ & $\begin{array}{c}100 \% \\
(2 / 2)\end{array}$ & $\begin{array}{c}100 \% \\
(6 / 6)\end{array}$ & $\begin{array}{c}\mathbf{5 0 . 0 \%} \\
(1 / 2)\end{array}$ \\
\hline False Exclusion & $\begin{array}{c}66.7 \% \\
(4 / 6)\end{array}$ & $\begin{array}{c}0 \\
(0 / 2)\end{array}$ & $\begin{array}{c}0 \\
(0 / 6)\end{array}$ & $\begin{array}{c}50.0 \% \\
(1 / 2)\end{array}$ \\
\hline
\end{tabular}

2.6 Conclusions for Elemental Analysis of Printing Inks

The utility of SEM-EDS for the discrimination of four types of printing inks (toner, inkjet, offset, and intaglio) was evaluated in this study. Additionally, the SEM-EDS results were compared to LA-ICP-MS, a more sensitive elemental technique. LA-ICPMS was capable of detecting many more minor and trace elements compared to SEMEDS, and therefore provided greater than $99.6 \%$ discrimination among the different samples within each of the four ink types. Moreover, LA-ICP-MS provided excellent overall correct association ( $>90 \%$ ) for ink samples known to originate from the same source. Finally, when toner and inkjet samples of the same color (cyan, magenta, yellow, or black) were compared, LA-ICP-MS was still able to provide discrimination greater than $97 \%$ within each color. 
However, unlike SEM-EDS, LA-ICP-MS is not routinely used in many forensic laboratories because of its high cost. Although less sensitive than LA-ICP-MS, SEMEDS was still capable of providing greater than $97 \%$ discrimination, as well as no false exclusions, for toner and intaglio samples. Moreover, SEM-EDS offers both imaging and elemental analysis capabilities, which is particularly important for toner samples since differences in the particle morphology can provide an additional means of differentiation. When comparing toners of the same color, greater than $94 \%$ of the samples were distinguishable. However, the technique was found to have limited utility for the analysis of inkjet and offset samples. Few elements were detected for the inkjet set, resulting in a relatively low discrimination $(\sim 78 \%)$. When comparing inkjet samples of the same color, the discrimination for cyan, magenta, and yellow inks improved slightly, while less than half of the black inkjets were distinguishable. Although more elements were detectable for the offset samples the contribution from the paper substrate led to a relatively poor discrimination ( 80\%). Interestingly, SEM-EDS provided some complementary information to LA-ICP-MS for the toner, inkjet, and offset samples. Several elements (e.g., K, Fe, Ca, S, Si, Cl) that are difficult to detect by LA-ICP-MS due to polyatomic interferences were easily detected by SEM-EDS and allowed for the differentiation of some pairs that were indistinguishable by LA-ICP-MS.

A subset of toner and ink samples was also analyzed using LIBS. While LIBS provided good discrimination $(>90 \%)$ using spectral overlay, the poor reproducibility of the technique resulted in poor correct association rates $(78.9 \%$ and $21.7 \%$ for inkjets and toners, respectively). In order to improve the association rates, a modified $\pm 4 \mathrm{SD}$ match criterion, similar to that used for LA-ICP-MS analysis of glass, was attempted for 
pairwise comparisons using element ratios. The match criterion resulted in good overall discrimination (> 95\%) and good correct association rates (> 89\%) for both inkjets and toners. Moreover, LIBS offered good discrimination for each individual ink color (CMYK): $>80 \%$ for inkjets and $>95 \%$ for toners. The results indicate that LIBS is an excellent alternative to SEM-EDS; it performs better for the analysis of inkjets (particularly black inkjets) and yields comparable results for toners. Moreover, LIBS provides advantages over LA-ICP-MS: it is less expensive, it requires less maintenance, and it is less complex to operate, while still offering comparable discrimination capabilities. Most forensic laboratories do not have LA-ICP-MS because of its cost. LIBS, on the other hand, is more likely to be implemented in forensic laboratories.

The final part of the ink project involved the development of a searchable ink database, FIACS. The database uses PLSDA and KNN to compare an unknown ink sample to a reference collection. Duplicate samples of the four ink types were used as test sets in order to evaluate the performance of the database. In general, LA-ICP-MS performed better than SEM-EDS, likely because of its greater discriminating power. LA-ICP-MS provided a correct association $>94 \%$ for all ink types when PLSDA and KNN were combined. However, inkjet and intaglio samples had limited duplicate samples (between 1 and 6 duplicates); a larger set of duplicates is necessary to estimate the correct classification rate of the FIACS database. SEM-EDS provided good association ( $\geq 95 \%)$ for offset and intaglio samples when PLSDA and KNN were combined. However, even after combining the two classification tools, SEM-EDS provided a correct association rate $\leq 76 \%$ for inkjet and toner samples. It should be noted that SEM-EDS had a limited number of replicate measurements ( 3 replicates per sample) because of the long sampling 
time ( $\sim 20$ minutes per replicate). On the other hand, LA-ICP-MS had 4-12 replicates, depending on the sample, because of the much shorter sampling time $(\sim 2$ minutes per replicate). A larger number of replicates is expected to improve the results since the algorithms will be able to model the data better. A larger reference collection would also be beneficial, which is why the user has the capability of continuously adding new ink samples to the reference collection set, expanding the FIACS database over time. 


\section{LA-ICP-MS ANALYSIS OF GLASS AND COLLECTION OF GLASS DATABASES}

\subsection{FIU Vehicle Glass Sample Collection and Preparation}

The FIU vehicle glass collection contains a total of 420 glass samples taken from the inner and outer windshield pane of 210 vehicles located at the M\&M Service and Salvage Yard in Ruckersville, Virginia. A complete list of all vehicles sampled is provided in Appendix B. As can be seen in Figure 41 B., windshield glass consists of two glass panes held together by a plastic film. The windshield glass was cut using a RHYNO laminated glass cutter. One to three large $(\sim 6$ by $8 \mathrm{~cm})$ glass pieces, shown in Figure $41 \mathrm{C}$, were collected depending on how much glass was available for a particular vehicle. Only vehicles with a legible VIN were sampled at the salvage yard; apart from the VIN, the make and model of each car was also cataloged. Whenever possible, a picture of the windshield sticker was taken (Figure 41 A.); this sticker discloses the glass manufacturer as well as the date of manufacture. Unfortunately, only 82 of the 210 vehicles sampled had this sticker intact, thus for most samples, the origin of the glass is unknown. While sampling the windshields, a sticker with the sample ID was placed on the outer windshield pane (Figure 41 C.) in order to differentiate the inner and outer pane. Once back at FIU, one large piece was fractured by pounding the surface with a pestle, which allowed smaller glass fragments to be peeled from the polymer film. Three small pieces (typically less than $1 \mathrm{~cm}^{2}$ ) were taken from the outer pane and the side of the pane that was not in contact with the polymer film was labeled with a black "." (Figure 41 D.); the same was done for the inner pane, though these were labeled with a purple " $\times$ " (Figure 41 E.). The side of the glass that was marked, either with a black "." or purple " $\times$," was the 
side analyzed using LA-ICP-MS. In some cases, this pane side corresponded with the float side (verified by the large ${ }^{118} \mathrm{Sn}$ signal).

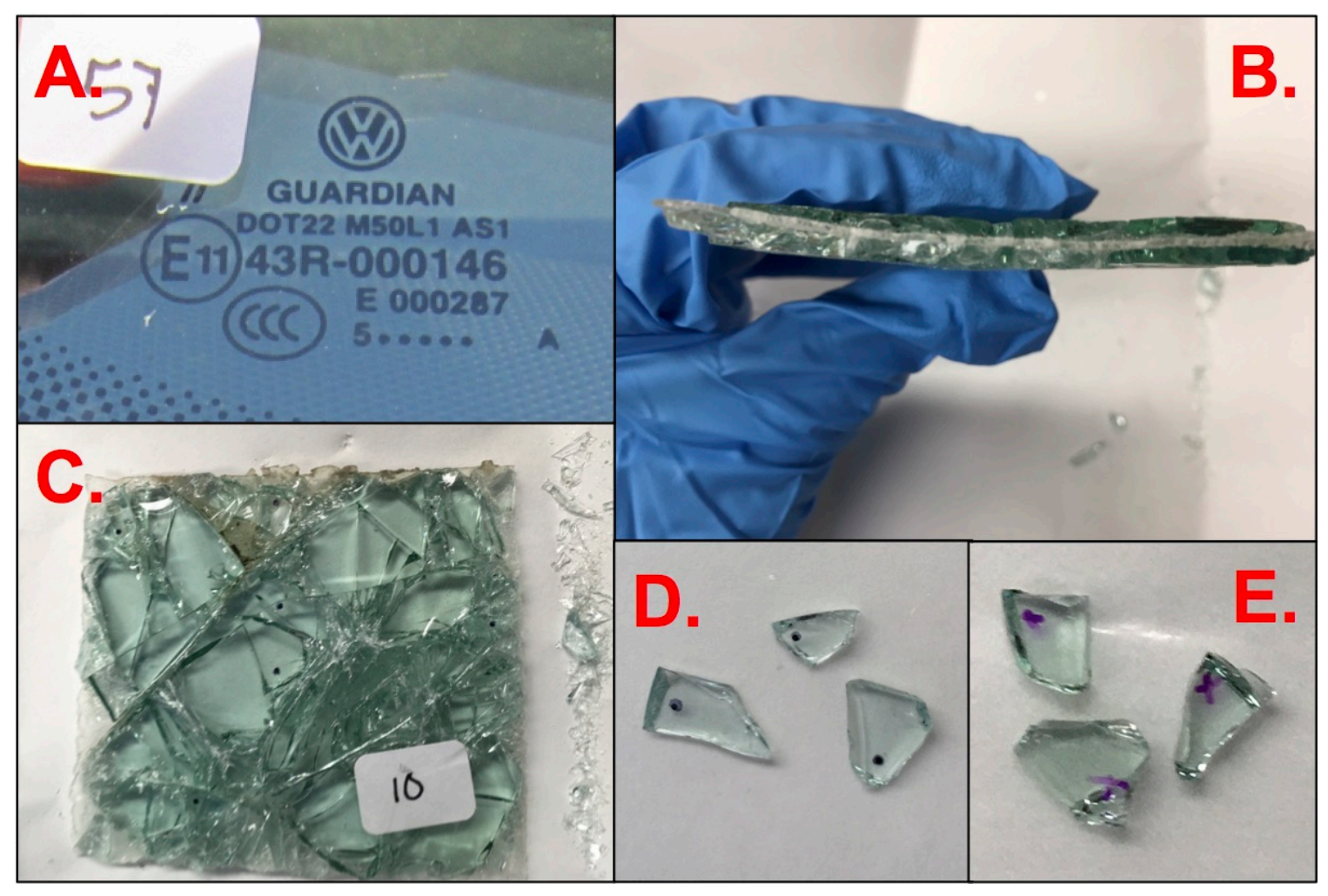

Figure 41 - Example of windshield sticker disclosing glass manufacturer, (B.) side view of laminated glass, (C.) example of large piece of glass cut from windshield, (D.) small glass fragments broken from outer windshield pane, and (E.) small glass fragments broken from inner windshield pane

For each of the three small fragments, 5 replicate measurements were collected, for a total of 15 replicates per sample. Each replicate analysis was named with an ID number indicating the vehicle number, followed by the fragment number $(1,2$, or 3$)$, then the pane side (inner or outer), and finally the replicate number (1-5). For example, the name “067.2 F3 Inner R4” indicates vehicle 067 (the number after the decimal point indicates which of the 3 large glass pieces from the windshield was sampled), fragment 3 , inner 
pane, and replicate 4. The name "192.3 F1 Outer R1" indicates vehicle 192 (third glass piece), fragment 1 , outer pane, and replicate 1.

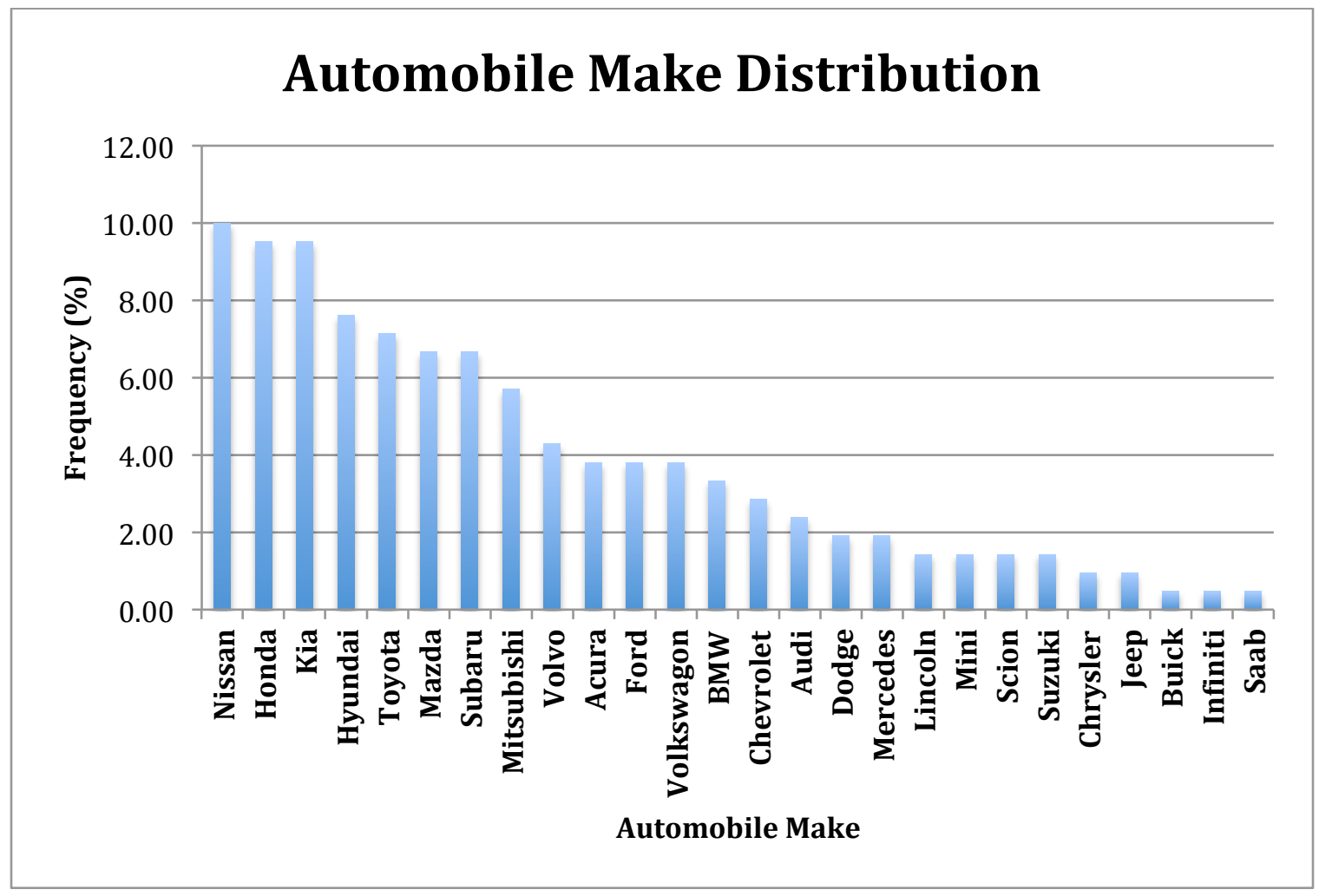

Figure 42 - Relative frequency of automobile make for windshield glass in FIU collection set

A total of 26 automobile makes are represented in the FIU vehicle collection set. The percent frequency for each automobile make is shown in Figure 42 . Over $40 \%$ of the collection set originated from just 5 vehicle makes: Nissan, Honda, Kia, Hyundai, and Toyota. Although these vehicle makes are popular, the two top-selling makes in the United States, Ford and Chevrolet, are underrepresented. Nevertheless, Toyota, Honda, and Nissan are top-selling brands and are well represented in the FIU collection.

Figure 43 shows the percent unit vehicle sales in the United States for each vehicle make; the data was compiled from the website "carsalesbase.com" and includes all unit sales 
between the years 2004 and 2017 (the represented years in the FIU collection set). Of course, this figure shows the distribution of vehicles across the entire United States; distributions may vary widely depending on location.

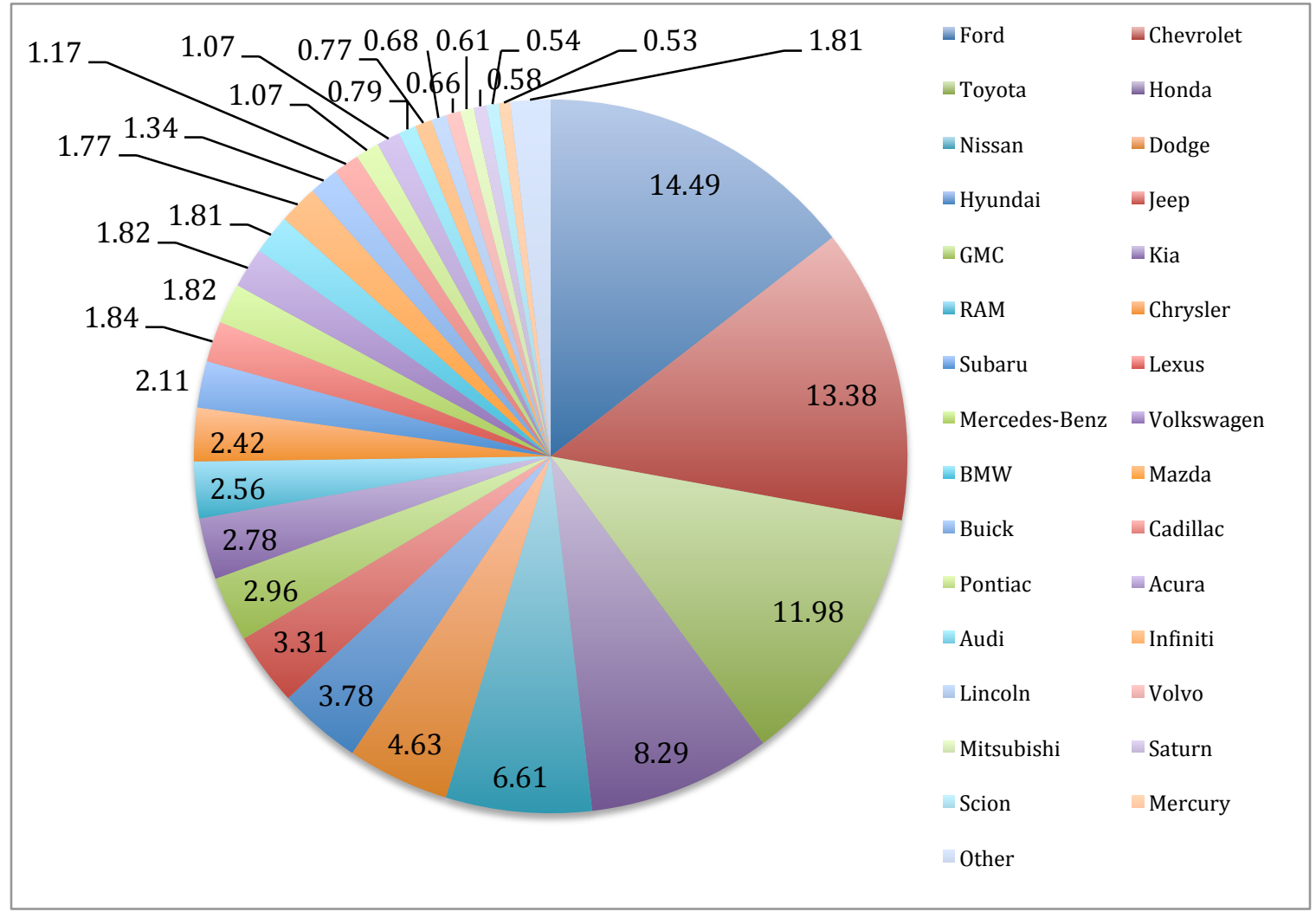

Figure 43 - Relative percent of unit sales in the United States for each automobile make

It should also be noted that, while this figure gives an idea of the frequency of vehicle makes within the US, it does not give information about the frequency of glass produced by a particular glass plant. Different vehicle manufacturers may obtain their glass from the same glass plant; additionally, each vehicle manufacturer may obtain glass from several different glass plants. However, the glass plant(s) used by the top-selling vehicle manufacturers are still expected to be the most prevalent in a given population. 
While vehicles between the years 2004 and 2017 were sampled, the majority of the vehicles $(\sim 42 \%)$ were manufactured between the years 2014 and 2016. Figure 44 shows the $\%$ frequency of vehicles manufactured in a particular year.

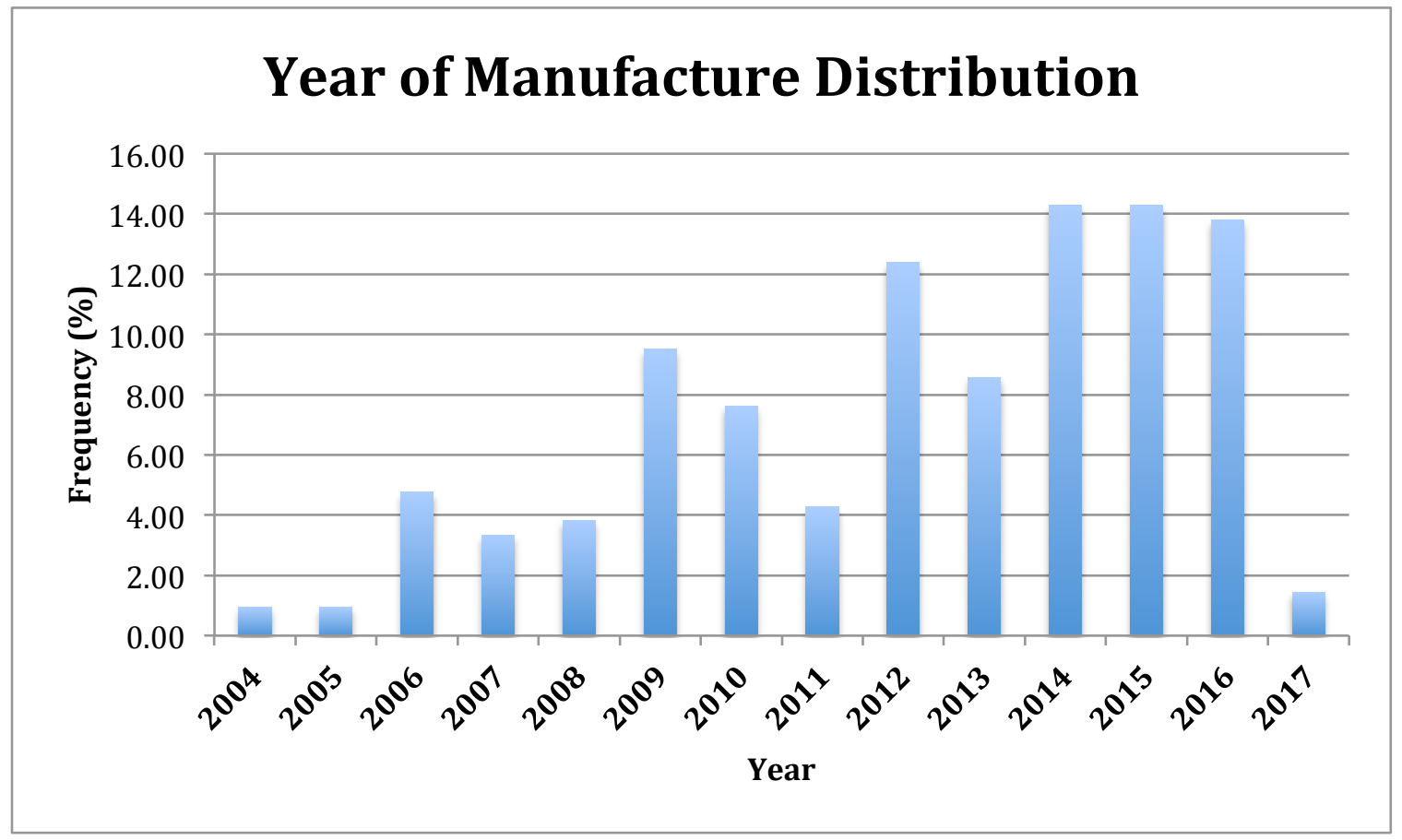

Figure 44 - Relative frequency of manufacturing year for windshield glass in FIU collection set

\subsection{Elemental Analysis of FIU Vehicle Glass using LA-ICP-MS}

The 17 isotopes listed in ASTM E2927 were monitored: ${ }^{7} \mathrm{Li},{ }^{25} \mathrm{Mg},{ }^{27} \mathrm{Al},{ }^{39} \mathrm{~K},{ }^{42} \mathrm{Ca},{ }^{49} \mathrm{Ti}$, ${ }^{55} \mathrm{Mn},{ }^{57} \mathrm{Fe},{ }^{85} \mathrm{Rb},{ }^{88} \mathrm{Sr},{ }^{90} \mathrm{Zr},{ }^{137} \mathrm{Ba},{ }^{139} \mathrm{La},{ }^{140} \mathrm{Ce},{ }^{146} \mathrm{Nd},{ }^{178} \mathrm{Hf}$, and $\mathrm{Pb}$ (average of ${ }^{206} \mathrm{~Pb}$, ${ }^{207} \mathrm{~Pb}$, and $\left.{ }^{208} \mathrm{~Pb}\right) .{ }^{115}$ Two additional isotopes were monitored, but not used for characterization purposes: ${ }^{29} \mathrm{Si}$ was used as the internal standard since $\mathrm{SiO}_{2}$ is present at high concentration $(\sim 72 \%)$ and ${ }^{118} \mathrm{Sn}$ was monitored to determine the float side of the glass pane. The isotopes were quantified using single-point calibration with the Glitter ${ }^{\mathrm{TM}}$ software (MacQuarie University, Australia). Float Glass Standard 2 (FGS 2) was used as 
the calibrator, while FGS 1 and NIST 1831 were analyzed daily to assess bias. Prior to analysis, a daily performance using the NIST 612 standard was performed to ensure that the instrument sensitivity was adequate and that doubly charged species as well as oxides were below 3\%. A ns-213 nm Nd:YAG laser (ESI New Wave Research, Portland OR USA) coupled to a quadrupole ELAN DRC II (Perkin Elmer LAS, Shelton CT USA) was used for analysis. The laser parameters for analysis were as follows: 100\% laser energy $(\sim 0.65 \mathrm{~mJ}), 10 \mathrm{~Hz}, 90 \mu \mathrm{m}$ spot size, and 60 -second dwell. The laser ablation parameters and the ICP-MS method were previously developed during two inter-laboratory studies in which FIU participated: the Natural Isotopes and Trace Elements in Criminalistics and Environmental Forensic (NITECRIME) and the Elemental Analysis Working group (EAWG). ${ }^{36,70,73,115,134}$

The typical sequence of analysis is shown in Table 24. In general, 8 vehicles were completed in one day (approximately a 12 hour run). This amounts to 48 glass fragments in one day (excluding the calibrator and controls): 3 fragments for each inner and outer pane of every vehicle. The calibrator (FGS 2) was analyzed at the beginning, middle, and end of the sequence in order to account for instrumental drift. NIST 1831 was analyzed in the first half of the sequence, while FGS 1 was analyzed in the second half of the sequence.

After analysis of the 420 glass samples (inner and outer panes from 210 vehicles), 40 randomly selected duplicates were reanalyzed in order to assess the correct association rate. All glass samples and selected duplicates were analyzed over a total of 40 days. 
Table 24 - Typical LA-ICP-MS analysis sequence for FIU vehicle glass collection

\section{Analysis Sequence}

FGS 2: calibrator

Sample 1: inner \& outer pane

Sample 2: inner \& outer pane

NIST 1831: control

Sample 3: inner \& outer pane

Sample 4: inner \& outer pane

FGS 2: calibrator

Sample 5: inner \& outer pane

Sample 6: inner \& outer pane

FGS 1: control

Sample 7: inner \& outer pane

Sample 8: inner \& outer pane

FGS 2: calibrator

\subsection{Performance of Control Samples}

Two control samples, FGS 1 and NIST 1831, were run daily in order to assess the accuracy of the single-point calibration using FGS 2. Five replicate measurements were collected for each control for every day of analysis. Table 25 shows the percent bias for all 39 days in which FGS 1 was analyzed, while Table 26 shows the percent bias for all 40 days in which NIST 1831 was analyzed. A bias between 10 and $15 \%$ is highlighted in yellow, while a bias greater than $15 \%$ is highlighted in red. Note that FGS 1 was not analyzed on one of the days, since there was insufficient argon gas for a full 12-hour run.

The bias is a measure of how close the experimental value is to the expected (reported or certified) value. The percent bias is calculated as follows:

$$
\% \text { Bias }=100 \times \frac{(\text { Reported Value }- \text { Experimental Value })}{\text { Reported Value }}
$$

For FGS 1, Li often had a bias between 10 and 15 percent indicating that the experimental concentration was often higher than the reported value of 6 parts per million 
(ppm). Fe, on the other hand, was often lower than the reported value of $580 \mathrm{ppm} . \mathrm{Rb}$ had a bias worse than $-10 \%$ for every single day of analysis; that is, $\mathrm{Rb}$ was always measured to be lower than the reported value of $8.6 \mathrm{ppm}$. This is likely an indication of an incorrect reported value either for the FGS 2 calibrator or the FGS 1 control. The issue with the reported value of $\mathrm{Rb}$ is further corroborated from the results of an inter-laboratory study (Chapter 5), in which all but one lab consistently obtained a lower concentration for $\mathrm{Rb}$ than is reported. The bias for $\mathrm{Pb}$ on Day 08 of analysis was at an extremely high $46 \%$. The high concentration for $\mathrm{Pb}$ on Day 08 was reproducible (i.e., there were no outliers in the data nor were there any spikes in the transient signal). It is unknown what caused this high $\mathrm{Pb}$ concentration on Day 08 for FGS 1, as the Pb concentration for both FGS 2 and NIST 1831 were within the usual limits. Moreover, two of the glass samples analyzed on Day 08 (045.1 Outer and 046.1 Inner) were reanalyzed as duplicates on Day 42 and 43, respectively. The originals run on Day 08 and the duplicates were not found to be significantly different using the ASTM 2927 match criterion (discussed in more detail in Chapter 4). ${ }^{115}$ Relatively high bias was observed for a few other elements, though these instances were rare.

For NIST 1831, both $\mathrm{Li}$ and $\mathrm{Pb}$ consistently gave an experimental concentration that was higher than the reported values of $5 \mathrm{ppm}$ and $1.8 \mathrm{ppm}$, respectively. This may be due to the low concentration of these elements, particularly for $\mathrm{Pb}$, which may be close to the method detection limit. Fe was consistently lower than the reported value, which may indicate an incorrect reported value. This is corroborated by an inter-laboratory study in which the 7 participants obtained a lower value than reported (average of $500 \mathrm{ppm}$ 
compared to the reported $608 \mathrm{ppm}) .{ }^{115}$ The results of the inter-laboratory are discussed in detail in Chapter 5.

For both controls, the relative standard deviation (RSD) of the five replicates for each day of analysis was below $10 \%$, with a few rare exceptions. The RSD is a measure of precision and is calculated as follows:

$$
\% R S D=100 \times \frac{\text { Standard Deviation }}{\text { Average }}
$$

RSDs greater than $10 \%$ were observed only for Li or Pb, particularly for NIST 1831 . Even so, the RSD for these exceptions was below 15\% for all but one instance. The slightly high RSD for these two elements may be because of their low concentration. Overall, however, good precision was observed for both FGS 1 and NIST 1831. 


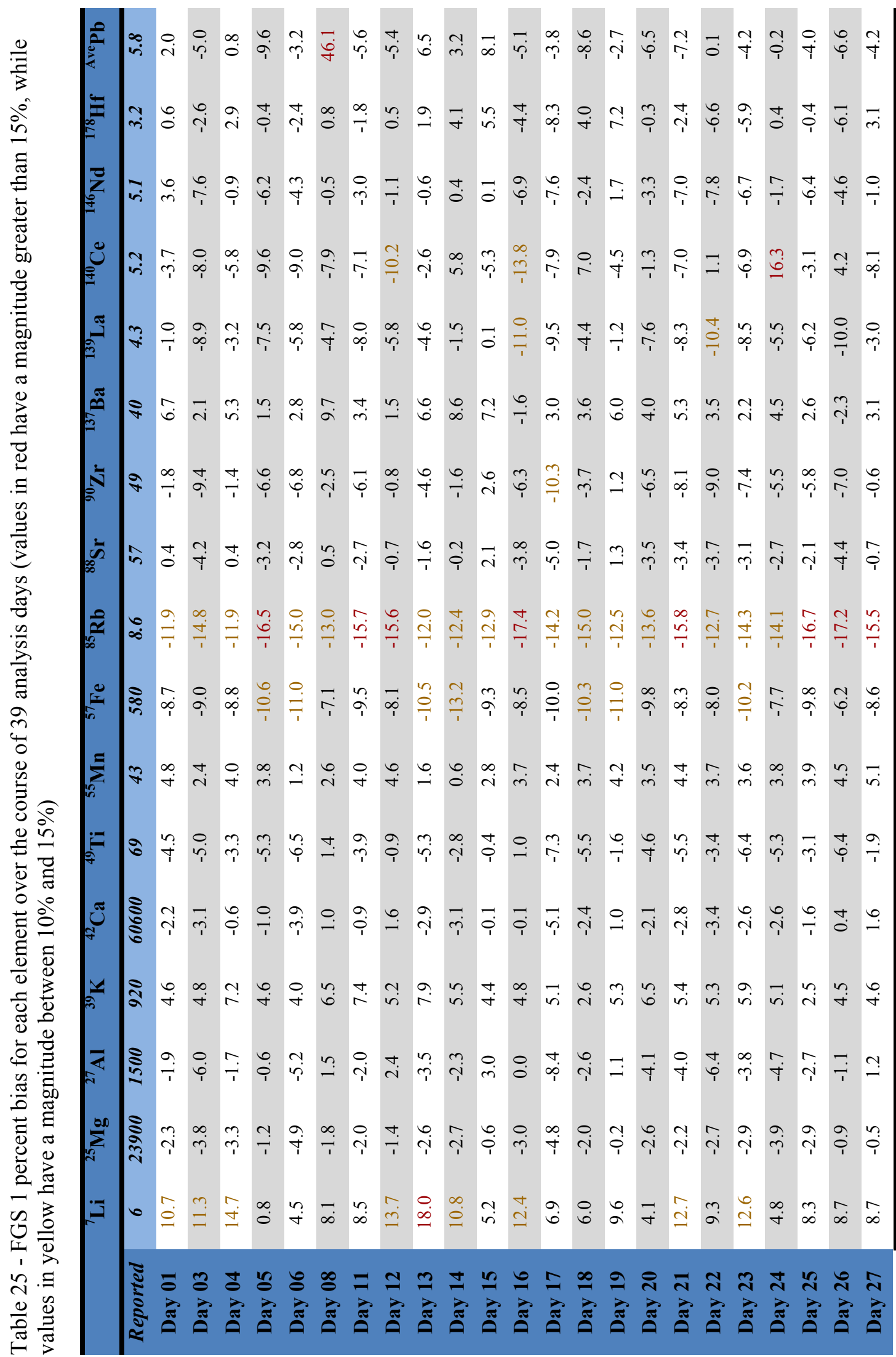




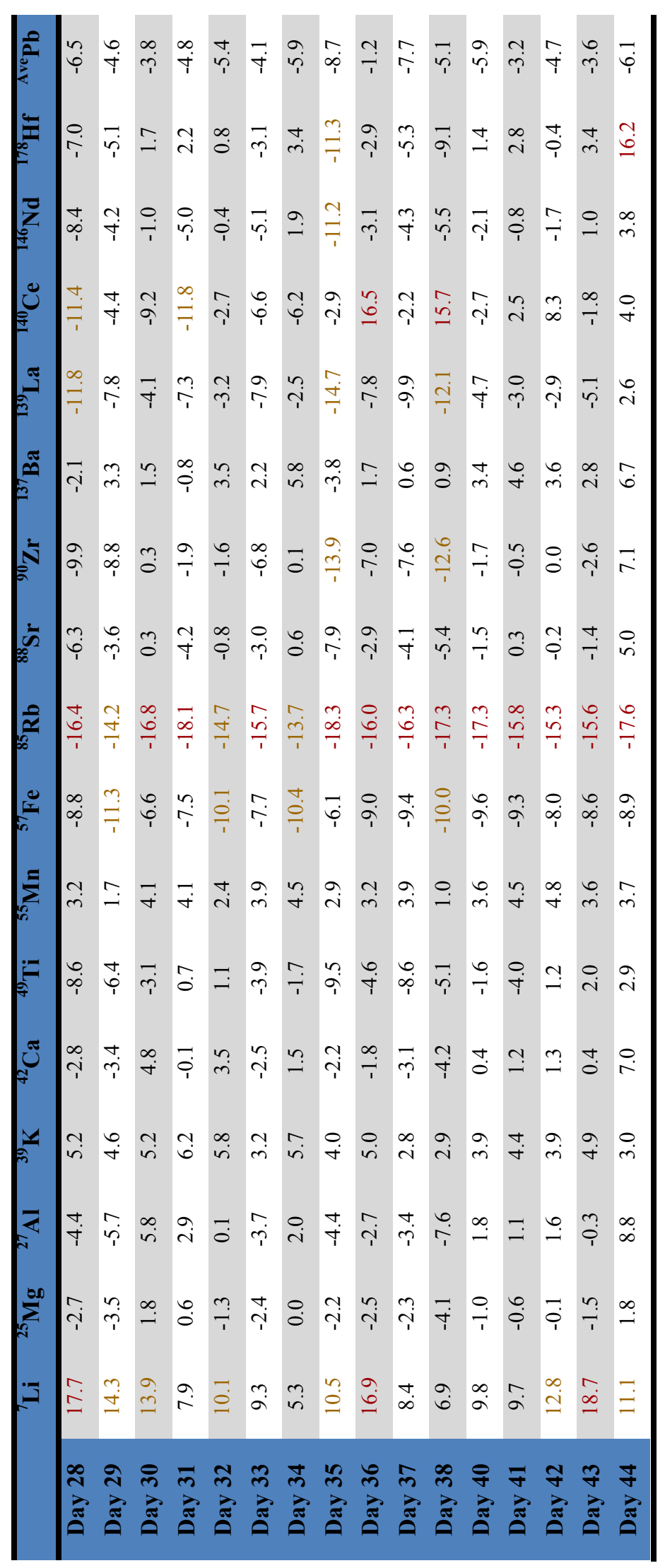




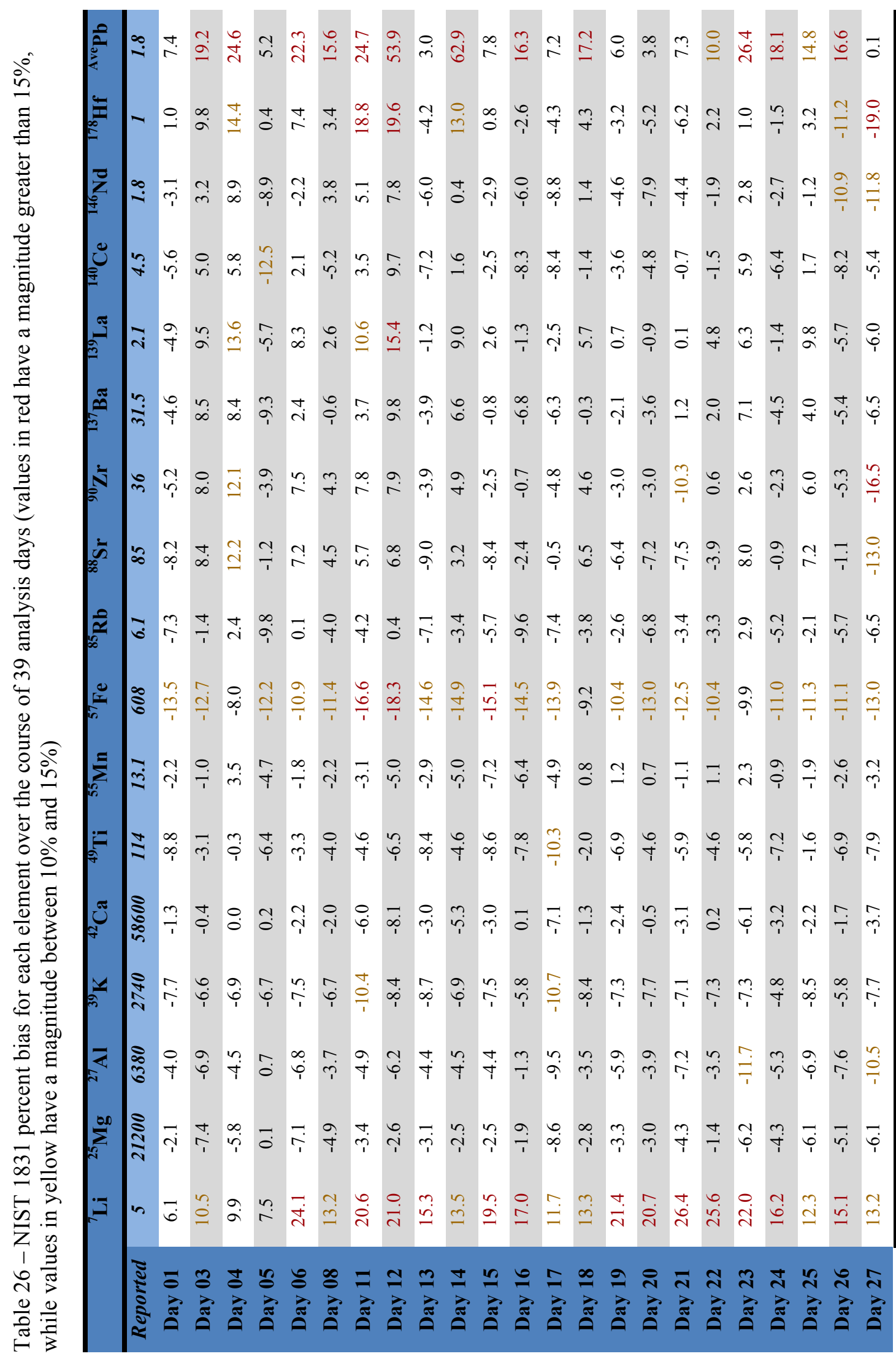




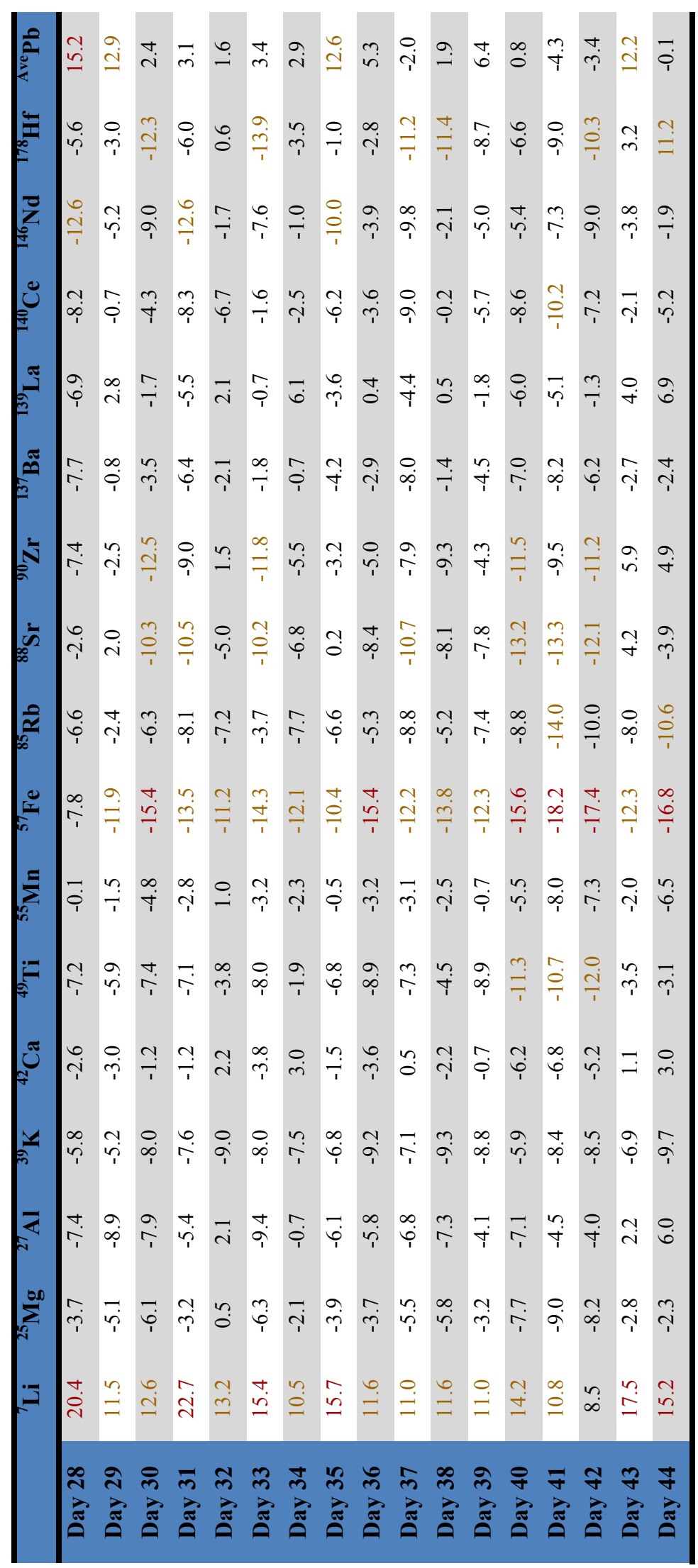


In order to assess variation of each element over time, a Levey-Jennings control chart was generated using RStudio (software version 1.0.143) for all elements measured. Figure 45 and Figure 46 show the control charts for FGS 1 and NIST 1831, respectively. The dashed green line reflects the overall average for all days of analysis. The dashed yellow and red lines indicate the \pm 2 and \pm 3 standard deviations, respectively. Average daily concentrations (shown as black points) between the yellow and red lines should be considered warnings, while concentrations past the red lines indicate a problem with the analysis.

For FGS 1 , most elements fell between $\pm 2 \mathrm{~s}$. However, on the final day of analysis, Day 44, several elements fell between the $+2 \mathrm{~s}$ and +3 s lines: $\mathrm{Mg}, \mathrm{Al}, \mathrm{Ca}, \mathrm{Sr}, \mathrm{Zr}, \mathrm{La}$, and $\mathrm{Hf}$. Although the instrument passed the daily performance criteria on this day, the fact that the concentration of several elements was higher than usual indicates a potential problem with the data collected on that day. On Day 08 , the average concentration for $\mathrm{Pb}$ was past the $+3 \mathrm{~s}$ line; this is the same day in which the bias for $\mathrm{Pb}$ was extremely high (discussed earlier). All other elements were within the $\pm 2 \mathrm{~s}$ lines for that day, thus only $\mathrm{Pb}$ seemed to be problematic. For NIST 1831, the Al concentration was past the $+3 \mathrm{~s}$ line for Day 44 , corroborating the results obtained for FGS 1 . Pb had one point past the +3 s lines (Day 14), but this was the only problematic element for that day. Overall, good performance can be seen for both controls over time, with the exception of a few instances with $\mathrm{Pb}$. Inspection of the data collected on Day 44 suggests that the problem for that day may have been due to the pulse/analog calibration of the ICP-MS detector. 


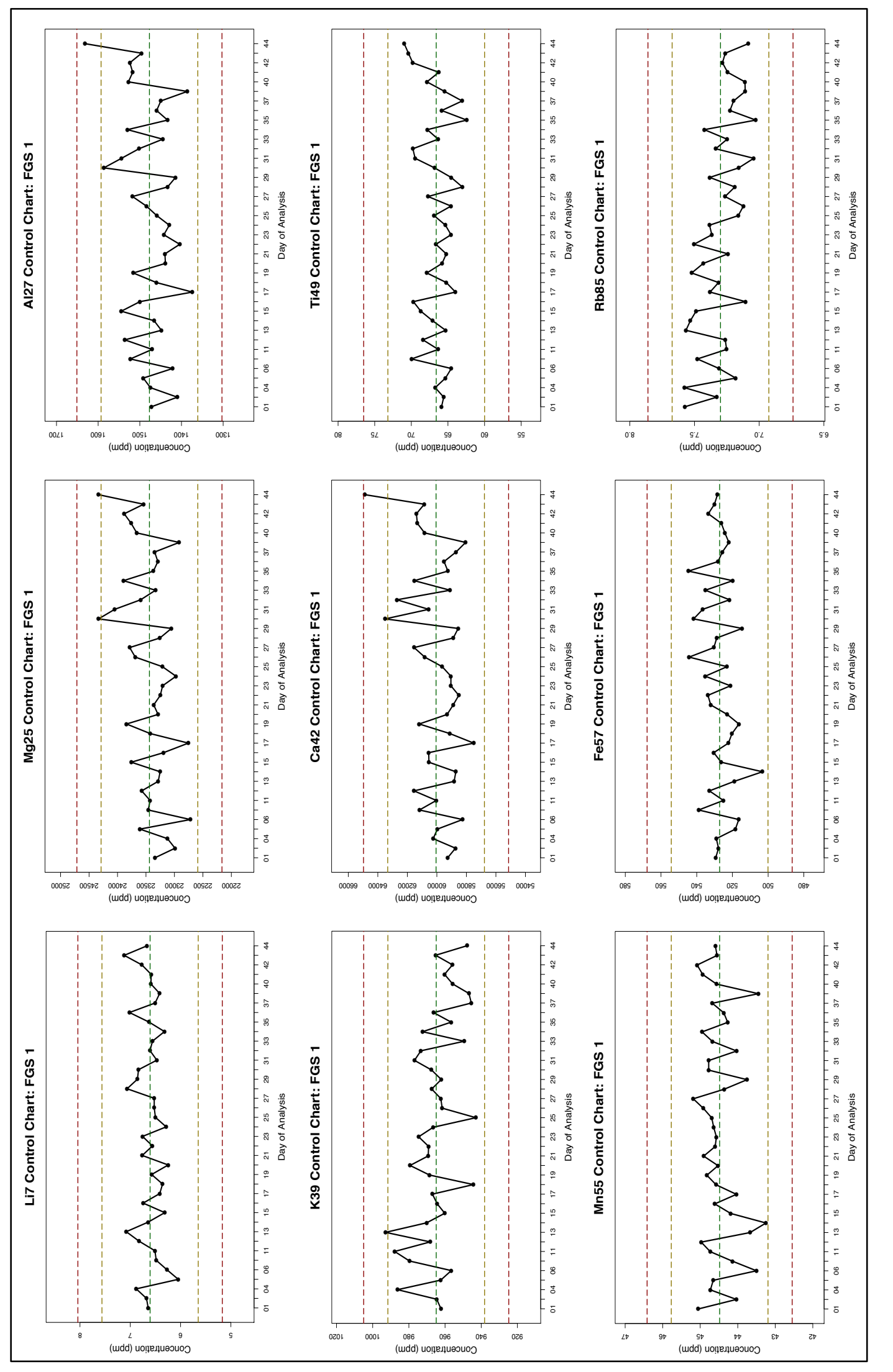

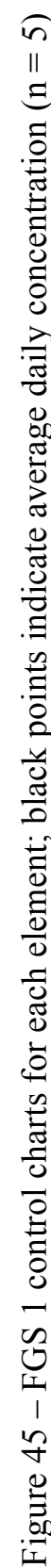




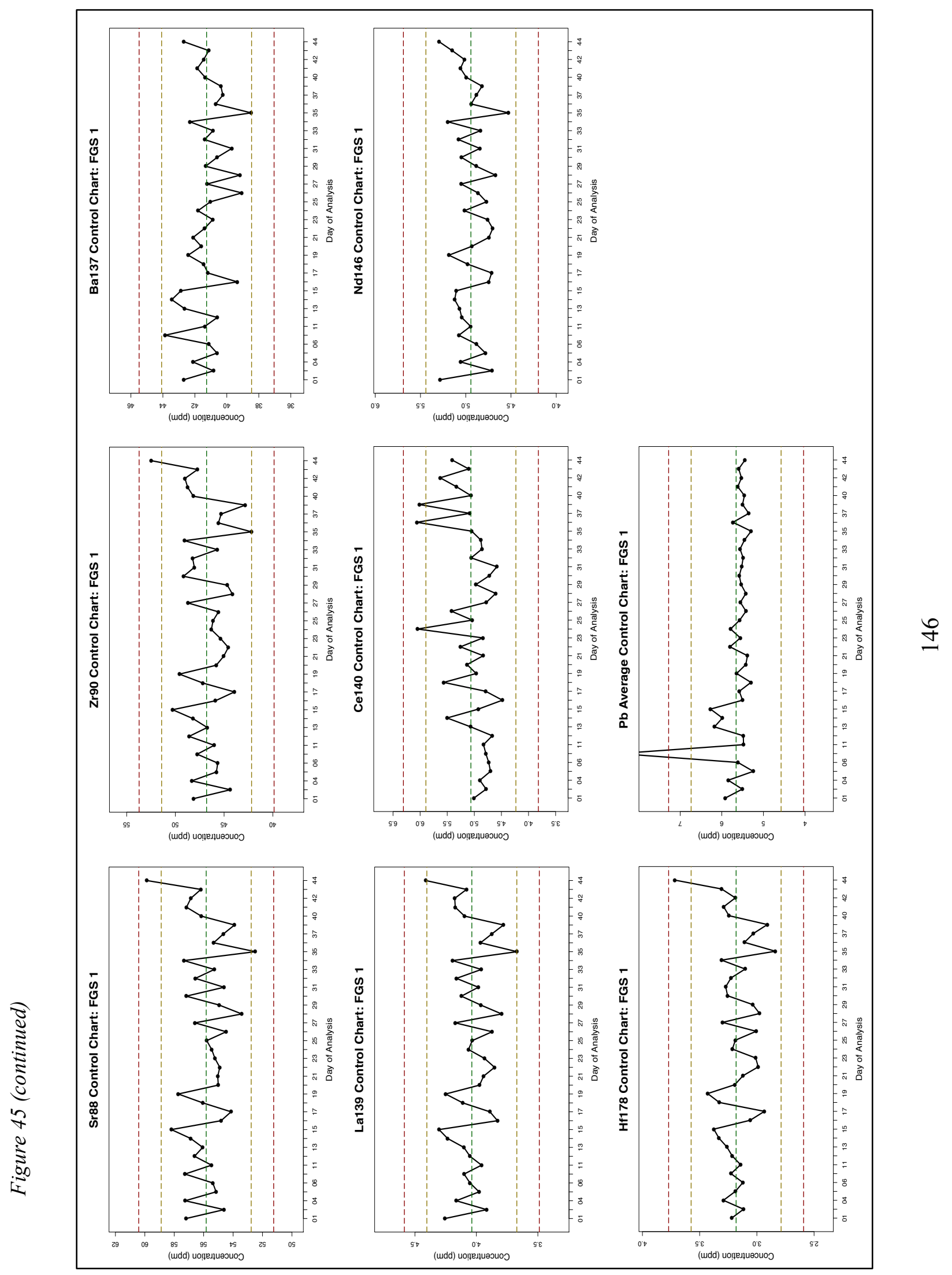




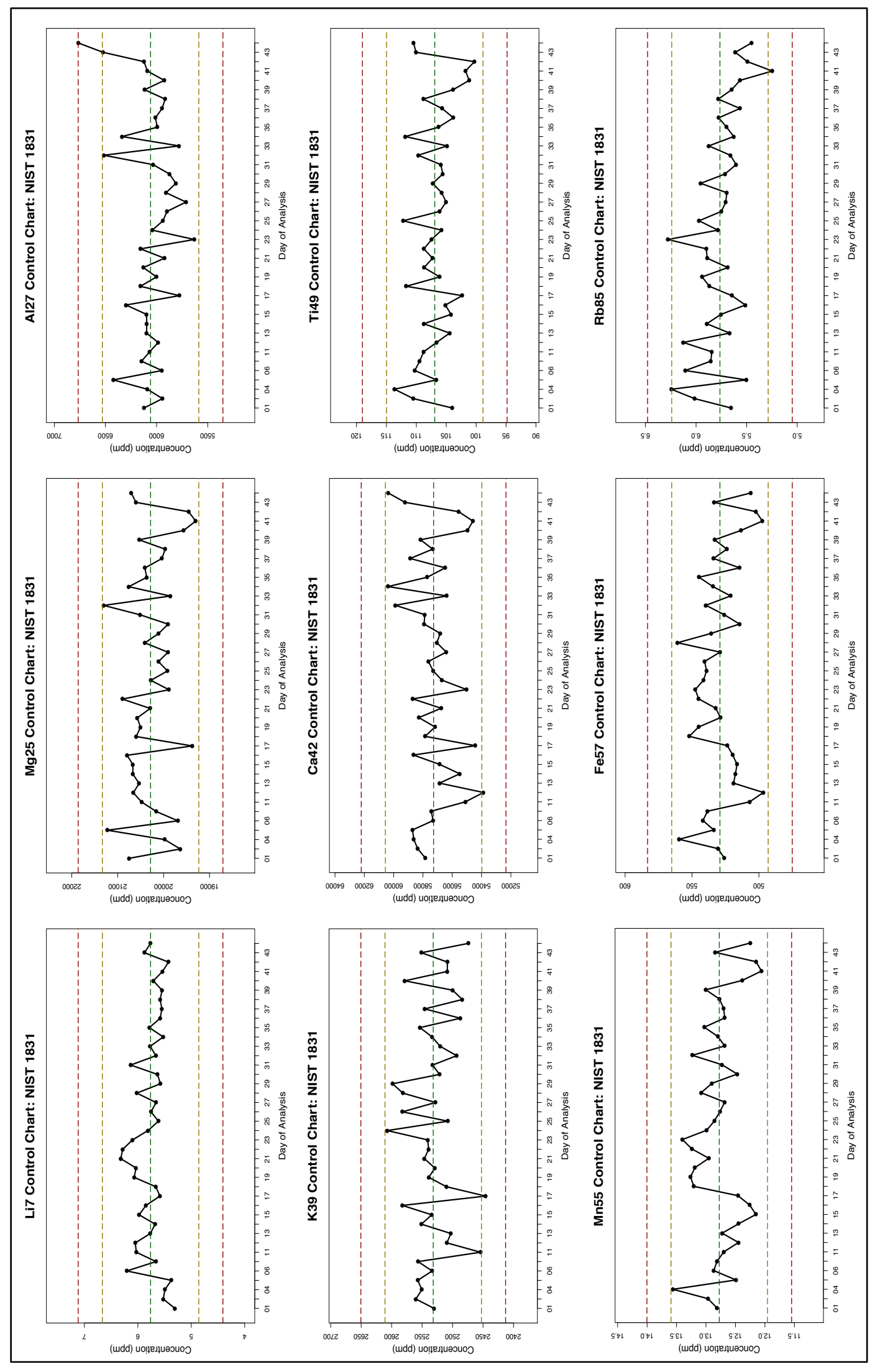

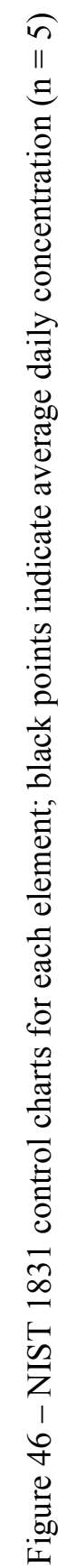




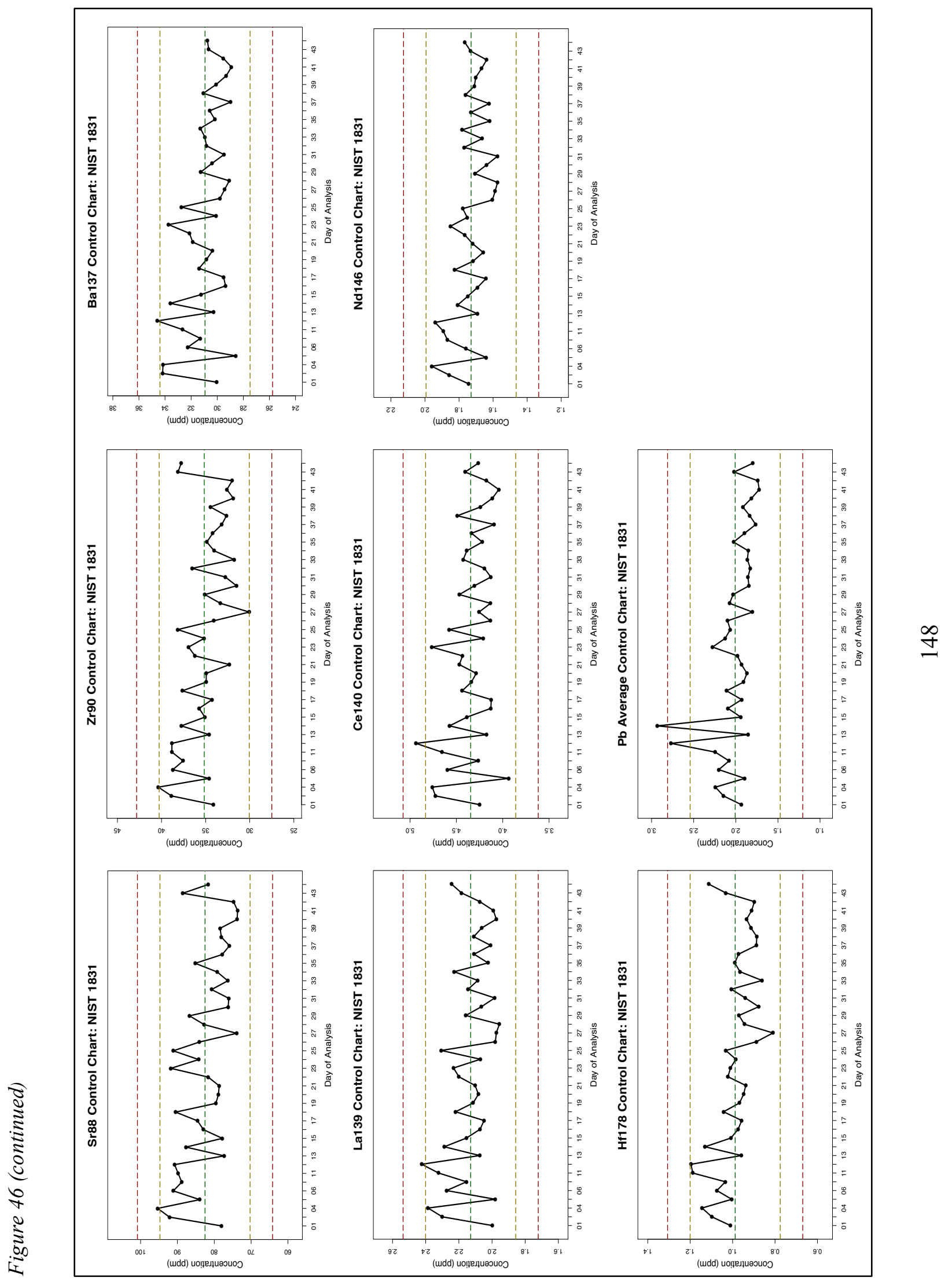


3.4 Figures of Merit and Descriptive Statistics for the FIU Vehicle Glass Database

\subsubsection{Limits of Detection}

In order to determine the method detection limit, a multi-point external calibration curve was generated for each element using the following glass standards: FGS 2, NIST 610, NIST 612, NIST 614, and NIST 1831. The standard FGS 1 was also analyzed in order to assess the accuracy of the calibration curves. The reference (certified or reported) values used for each calibration standard are listed in Table 27. Reference concentrations were obtained from the GeoRem Database (available online) for Geological and Environmental Reference Materials or from ASTM 2927. ${ }^{115}$

Table 27 - Reference concentration (ppm) used for each calibration standard

\begin{tabular}{cccccc}
\hline & FGS 2 & NIST 610 & NIST 612 & NIST 614 & NIST 1831 \\
\hline${ }^{7} \mathbf{L i}$ & 29 & 468 & 41.54 & 1.69 & 5 \\
${ }^{25} \mathbf{M g}$ & 23400 & 432 & 77.44 & 33.8 & 21200 \\
${ }^{27} \mathrm{Al}$ & 7400 & 10319 & 11164 & 10796 & 6380 \\
${ }^{39} \mathrm{~K}$ & 4600 & 464 & 66.26 & 30 & 2740 \\
${ }^{42} \mathrm{Ca}$ & 59300 & 81476 & 85262.5 & 85049 & 58600 \\
${ }^{49} \mathrm{Ti}$ & 326 & 452 & 48.11 & 3.61 & 114 \\
${ }^{55} \mathrm{Mn}$ & 221 & 444 & 38.43 & 1.42 & 13.1 \\
${ }^{57} \mathbf{F e}$ & 2600 & 458 & 56.33 & 18.8 & 608 \\
${ }^{85} \mathrm{Rb}$ & 35 & 425.7 & 31.63 & 0.855 & 6.11 \\
${ }^{88} \mathrm{Sr}$ & 253 & 515.5 & 78.4 & 45.8 & 85 \\
${ }^{90} \mathrm{Zr}$ & 223 & 448 & 35.99 & 0.848 & 36 \\
${ }^{137} \mathrm{Ba}$ & 199 & 452 & 37.74 & 3.2 & 31.5 \\
${ }^{139} \mathbf{L a}$ & 18 & 440 & 35.77 & 0.72 & 2.12 \\
${ }^{140} \mathrm{Ce}$ & 23 & 453 & 38.35 & 0.813 & 4.54 \\
${ }^{146} \mathrm{Nd}$ & 25 & 430 & 35.24 & 0.752 & 1.8 \\
${ }^{178} \mathbf{H f}$ & 15 & 435 & 34.77 & 0.711 & 0.96 \\
${ }^{\mathbf{A v e}} \mathbf{P b}$ & 24 & 426 & 38.86 & 2.32 & 1.8 \\
\hline
\end{tabular}


For each element, the transient signal was integrated and subsequently divided by the width of the integration to obtain counts per second (cps); the same was done for the blank signal. Integration was accomplished using Plot2 (software version 2.2.10). Then the blank cps was subtracted from the signal cps. Finally, each isotope was normalized to the internal standard, ${ }^{29} \mathrm{Si}$. This normalized cps was plotted against the reported values shown in Table 27. All calibration curves were plotted using RStudio (software version 1.0.143).

The calibration curves for each element showed excellent linearity $\left(R^{2}>0.99\right)$, with the exception of $\mathrm{Al}\left(\mathrm{R}^{2}=0.954\right)$ and $\mathrm{Ca}\left(\mathrm{R}^{2}=0.968\right)$. For both $\mathrm{Al}$ and $\mathrm{Ca}$, several of the calibration standards have similar concentrations thus yielding a 2-point or 3-point curve, rather than a 5-point curve, and limiting the concentration range of the calibration curves. Two trials were performed for each element and the linearity for both trials were similar. Figure 47 shows the Trial 1 calibration curves for a selection of elements. The standards used as calibrators are shown in blue, with a best-fit linear regression line added, while the calibration check standard (FGS 1) is plotted in red. Each plot shows the linear equation for the best-fit linear trend line, the $\mathrm{R}^{2}$ value, the limit of detection (LOD), and the concentration of the calibration check (FGS 1) using the best-fit linear equation. The LOD was calculated as follows:

$$
L O D=\frac{3 \times s}{m}
$$

In the equation above, $s$ is the standard error of the regression line and $m$ is the slope of the regression line. Table 28 shows the Trial 1 and Trial 2 LOD for each element. The table also lists the FGS 1 reported concentration for each element as well as the bias for 
both trials. For the most part, bias was within acceptable limits $(<15 \%)$. Rb showed a high bias though as discussed previously, this element is suspected of having an incorrect reported value. The calibration curve for $\mathrm{Rb}$ (Figure 47) showed excellent linearity and the calibration check, FGS 1, fell on the linear regression line. $\mathrm{La}, \mathrm{Ce}, \mathrm{Nd}, \mathrm{Hf}$, and $\mathrm{Pb}$, all also had high bias. However, these five elements are present at low concentrations that are near or below the LOD. For the most part, the LODs between the two trials were in agreement. However, a few elements showed a marked difference in LOD between the two trials: $\mathrm{Mg}, \mathrm{Mn}$, and $\mathrm{Ba}$.

Table 28 - Limit of detection (LOD) in parts per million (ppm) and FGS 1 bias for each element

\begin{tabular}{cccccc}
\hline & $\begin{array}{c}\text { LOD: } \\
\text { Trial } \mathbf{1}\end{array}$ & $\begin{array}{c}\text { LOD: } \\
\text { Trial 2 }\end{array}$ & $\begin{array}{c}\text { FGS1 } \\
\text { Reported }\end{array}$ & $\begin{array}{c}\text { FGS1 Bias: } \\
\text { Trial 1 }\end{array}$ & $\begin{array}{c}\text { FGS1 Bias: } \\
\text { Trial 2 }\end{array}$ \\
\hline${ }^{7} \mathbf{L i}$ & 5.9 & 5.19 & 6 & 10.00 & 11.67 \\
${ }^{25} \mathbf{M g}$ & 859 & 2068 & 23900 & -3.02 & -2.35 \\
${ }^{27} \mathbf{A l}$ & 2373 & 2317 & 1500 & -7.32 & 13.60 \\
${ }^{39} \mathbf{K}$ & 336 & 417 & 920 & 10.22 & 13.46 \\
${ }^{42} \mathbf{C a}$ & 12289 & 13480 & 60600 & -3.75 & 0.26 \\
${ }^{49} \mathbf{T i}$ & 34.6 & 47.8 & 69 & -1.84 & 2.75 \\
${ }^{55} \mathbf{M n}$ & 17.1 & 3.6 & 43 & 3.07 & 1.19 \\
${ }^{57} \mathbf{F e}$ & 100.6 & 112.5 & 580 & -5.29 & -6.86 \\
${ }^{85} \mathbf{R b}$ & 3.9 & 2.9 & 8.6 & -18.95 & -10.23 \\
${ }^{88} \mathbf{S r}$ & 17.2 & 19.6 & 57 & 0.42 & 5.84 \\
${ }^{90} \mathbf{Z r}$ & 23.7 & 28.7 & 49 & -7.41 & -7.65 \\
${ }^{137} \mathbf{B a}$ & 14.0 & 29.4 & 40 & 2.73 & 10.70 \\
${ }^{139} \mathbf{L a}$ & 5.0 & 5.4 & 4.3 & 13.49 & 20.70 \\
${ }^{140} \mathbf{C e}$ & 2.4 & 3.9 & 5.2 & 26.54 & 19.42 \\
${ }^{146} \mathbf{N d}$ & 5.5 & 6.3 & 5.1 & 14.71 & 20.59 \\
${ }^{178} \mathbf{H f}$ & 4.8 & 3.6 & 3.2 & 24.38 & 14.06 \\
${ }^{\mathbf{A v}} \mathbf{P b}$ & 3.7 & 6.7 & 5.8 & 3.79 & 18.45 \\
\hline
\end{tabular}



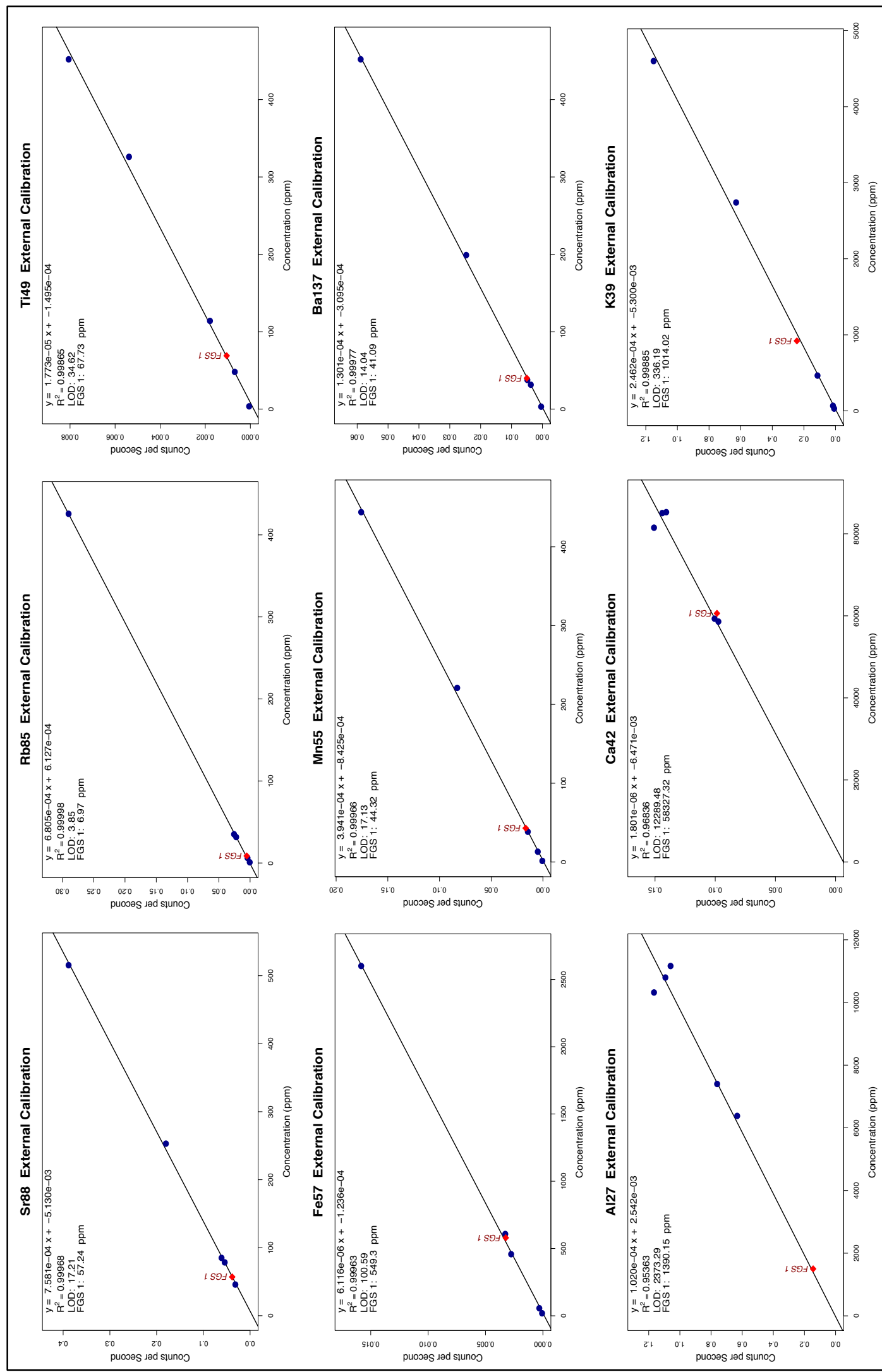

กี 


\subsubsection{Descriptive Statistics for FIU Vehicle Database}

In order to assess the spread of each variable (element) in the FIU vehicle database, a box and whisker plot was produced (Figure 48). In the plot, the $\log _{10}$ of the concentration was used so that all elements could be plotted on a smaller scale.

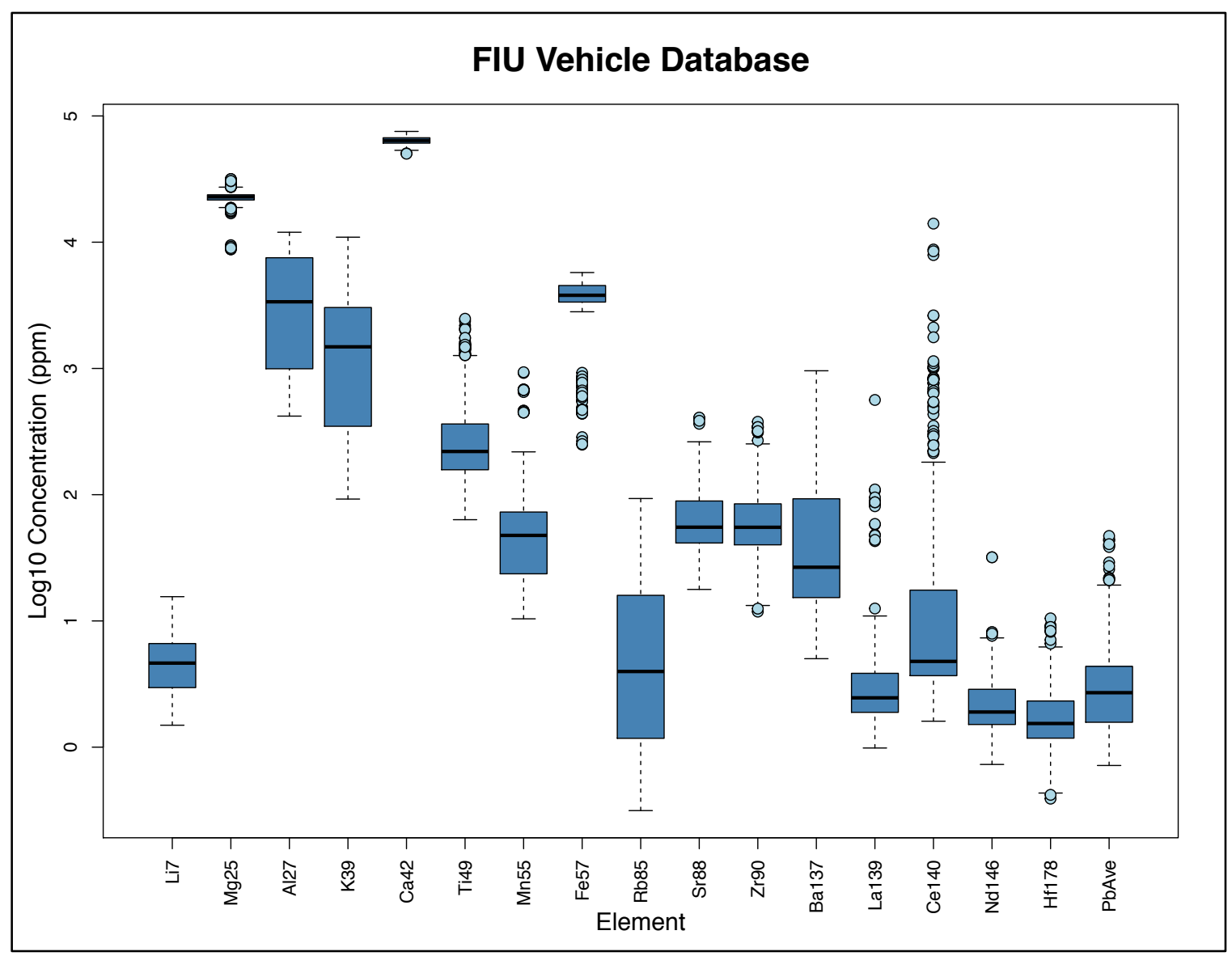

Figure 48 - Box and whisker plot for FIU vehicle database

The horizontal black line across each box represents the median value, while the upper and lower limits of the box are the upper and lower quartiles $\left(75^{\text {th }}\right.$ and $25^{\text {th }}$ percentile), respectively. The upper and lower whiskers are calculated as follows:

$$
\text { Upper whisker }=Q 3+1.5 \times I Q R
$$




\section{Lower whisker $=Q 1-1.5 \times I Q R$}

In the equations above, $Q 3$ is the upper quartile (i.e., the upper limit of the box), $Q 1$ is the lower quartile (i.e., the lower limit of the box), and $I Q R$ is the box length $(Q 3-Q 1)$. If the calculation of the upper whisker, using the formula above, is greater than the maximum value in the database, then the upper whisker is set as the maximum value; likewise, if the calculation of the lower whisker is less than the minimum value in the database, then the lower whisker is set as the minimum value. The light blue points that fall outside the whisker limits are extreme values that extend beyond the majority of the data for each particular element.

Some elements (e.g., $\mathrm{Mg}$ and $\mathrm{Ca}$ ) show little variation across all database samples, as indicated by the small range of the box. Other elements (Al, K, Rb) have much wider spreads. Fe has many extreme values that are approximately an order of magnitude lower than the rest of the data. Ce has the opposite trend: the majority of samples have a low $\mathrm{Ce}$ concentration, but many extreme values have a concentration that is two or three orders of magnitude higher; a similar trend is seen for La. The remaining elements have a relatively moderate spread with few, or no, extreme values.

In addition to the spread of each variable, the correlation between variables was investigated, shown in Figure 49. Hf and $\mathrm{Zr}$ are nearly perfectly correlated (positive correlation of 0.996 ), thus it may not be necessary to include both for the purpose of discrimination. $\mathrm{K}$ and $\mathrm{Al}$ and $\mathrm{Ba}$ and $\mathrm{Pb}$ are highly correlated as well (each pair has a positive correlation greater than 0.8 ). Other element pairs with fairly high correlation (positive correlation greater than 0.7 ) include: $\mathrm{Al}$ and $\mathrm{Rb}, \mathrm{K}$ and $\mathrm{Ba}$, and $\mathrm{La}$ and $\mathrm{Nd}$. 


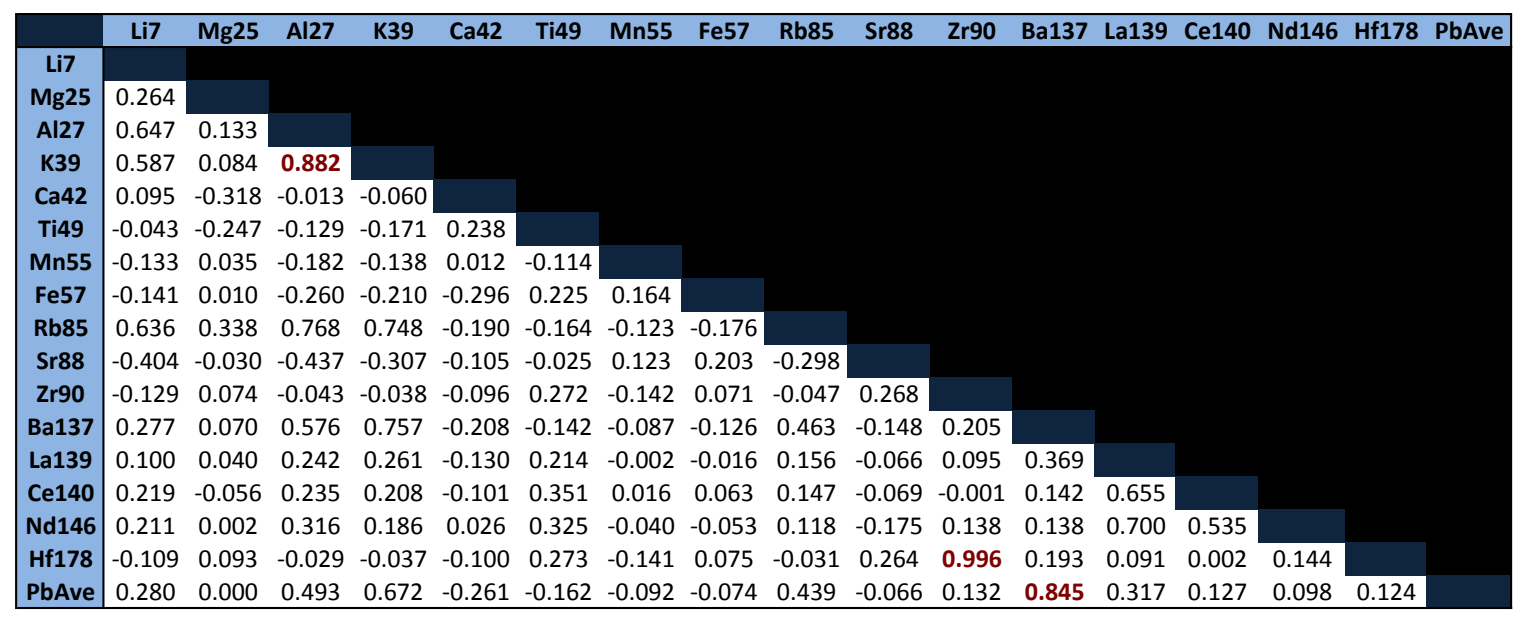

Figure 49 - Correlation between elements for FIU vehicle database

\subsection{Description of Additional Collected Datasets}

Apart from the FIU Vehicle database discussed above, several additional LA-ICP-MS databases were collected for interpretation purposes (Chapter 4). All of the databases subsequently described contain glass samples that were provided to the Bundeskriminalamt (BKA) laboratory in Germany for analysis. The BKA then kindly shared these datasets with FIU. Typically, the BKA analyzes the following 18 isotopes: ${ }^{7} \mathrm{Li},{ }^{23} \mathrm{Na},{ }^{25} \mathrm{Mg},{ }^{27} \mathrm{Al},{ }^{39} \mathrm{~K},{ }^{42} \mathrm{Ca},{ }^{49} \mathrm{Ti},{ }^{55} \mathrm{Mn},{ }^{57} \mathrm{Fe},{ }^{85} \mathrm{Rb},{ }^{88} \mathrm{Sr},{ }^{90} \mathrm{Zr},{ }^{137} \mathrm{Ba},{ }^{139} \mathrm{La},{ }^{140} \mathrm{Ce}$, ${ }^{146} \mathrm{Nd},{ }^{178} \mathrm{Hf}$, and ${ }^{208} \mathrm{~Pb}$ (as well as the internal standard, ${ }^{29} \mathrm{Si}$ ). However, ${ }^{23} \mathrm{Na}$ was eliminated from all datasets since it is not included in the element menu listed in the United States standard, ASTM E2927. ${ }^{115}$ All samples were analyzed with a total of 6 replicate measurements each. After LA-ICP-MS analysis, signal processing was accomplished with the Glitter ${ }^{\mathrm{TM}}$ software. The LA-ICP-MS instrumental parameters changed over time, but were in accordance with the relevant literature. ${ }^{37,73,115}$ 


\subsubsection{BKA Casework Database}

The BKA Casework Database is comprised of 385 glass samples that were submitted as part of a case (both known and questioned samples). These samples were analyzed over a long period of time: from 2005 to 2016. The casework database includes an assortment of glass types: float glass, container glass, pre-float window glass, etc. As such, the assumption that $\mathrm{SiO}_{2}$ is present at $\sim 72 \%$ may not be valid.

As for the FIU vehicle database, a box and whisker plot was produced for the BKA casework database. Compared to the FIU database, the BKA database exhibits more extreme values. This is not surprising since the BKA database consists of many types of glass samples, while the FIU database only includes float glass taken from automobile windshields. Furthermore, the FIU database contains many samples from vehicles of the same make, model, and year of manufacture; samples from similar vehicles are expected to have similar elemental profiles.

For the BKA casework database, $\mathrm{Zr}$ and $\mathrm{Hf}$ were found to be perfectly correlated (correlation of 1.0). Compared to the FIU database, there were many more element pairs that had a high correlation ( $\geq 0.9$ ), including: $\mathrm{Li}$ and $\mathrm{Ti}, \mathrm{Li}$ and $\mathrm{Zr}$, Li and $\mathrm{Hf}, \mathrm{Ti}$ and $\mathrm{Zr}$, Ti and Hf, $\mathrm{La}$ and $\mathrm{Ce}, \mathrm{La}$ and $\mathrm{Nd}$, and $\mathrm{Ce}$ and $\mathrm{Nd}$. 


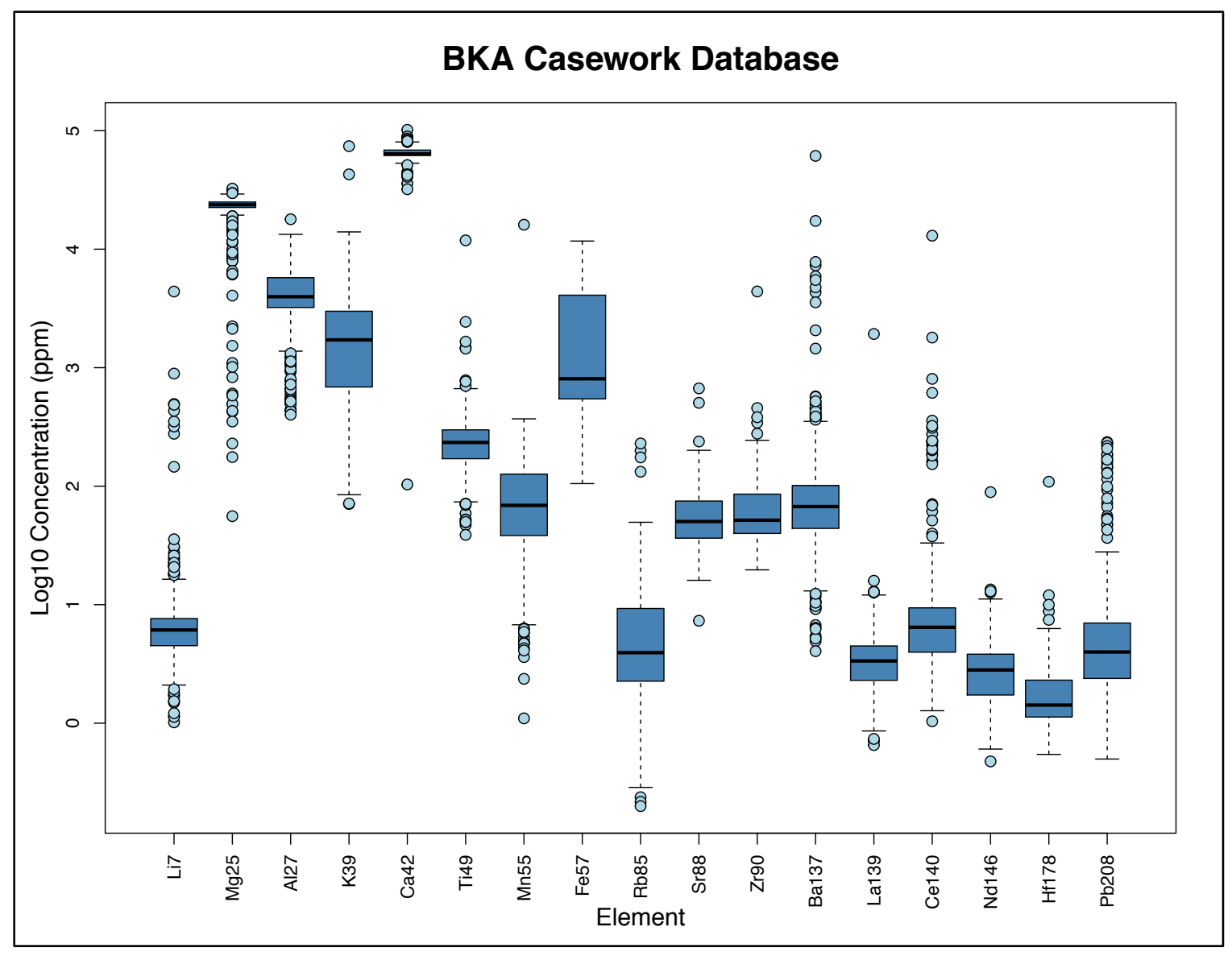

Figure 50 - Box and whisker plot for BKA casework database

The remaining collected datasets will only briefly be discussed since they are smaller datasets that are useful as test sets, rather than training sets (more detail can be found in Chapter 4).

\subsubsection{BKA Same Pane}

The BKA Same Pane consists of 34 glass fragments that all originated from a single pane provided by the FBI. These 34 fragments were analyzed a total of 44 times over a period 
of 11 days. Since all fragments originated from the same pane, all samples in this dataset are expected to be associated.

\subsubsection{BKA Float Glass}

This dataset includes 62 float glass samples that are part of the BKA reference collection. Unlike the other datasets discussed, the glass manufacturing plant for these samples is known. Glass from this set originates from one of eight corporations: Pilkington, Asahi Glass Co., Saint Gobain, Guardian Industries, PPG, Ford Glass Division, Central Glass Co., or Nippon Sheet Glass Co.

\subsubsection{NFI Daily Samples}

This set includes 41 samples provided by the Netherlands Forensic Institute (NFI) and analyzed by the BKA. All samples were produced within the same (undisclosed) manufacturing plant between June $21^{\text {st }} 2005$ and July $31^{\text {st }} 2005$. Since the chemical composition of the glass changes over time within a manufacturing plant, it is expected that samples produced closer together should be more similar than those produced further apart in time.

\subsubsection{FBI Vehicle Glass}

This set contains 151 vehicle glass samples provided by the FBI and analyzed by the BKA. This set includes vehicles that were manufactured between 1998 and 2010 from 17 automobile makes. In some cases, glass from the same vehicle but different windows 
(windshield, front driver, passenger, etc.) was sampled. Thus, these glass samples may have similar elemental profiles.

\subsubsection{Guardia Civil Vehicle Glass}

This dataset is comprised of 38 vehicle glass samples provided by the Guardia Civil in Spain and analyzed by the BKA. The set includes vehicles that were manufactured between 1989 and 2006 from 20 automobile makes. Like the FBI Vehicle Glass set, there were some cases in which different windows were sampled from the same vehicle.

\subsubsection{BKA Container Glass Set}

The final dataset is a small collection of 20 container glass samples from 9 glass manufacturers in Germany. The set includes a variety of products: wine bottles, champagne bottles, beer bottles, soda bottles, and glass jars.

\subsection{Conclusions for LA-ICP-MS Analysis of Glass and Collection of Glass Databases}

In this chapter, a detailed description of the FIU Vehicle Glass database, including sample collection, preparation, and LA-ICP-MS analysis, was discussed. The performance of the two daily controls, FGS 1 and NIST 1831, was evaluated. Good precision and bias $(<15 \%)$ was obtained for most elements; the few exceptions were likely due either to low concentration $(\mathrm{Li}$ and $\mathrm{Pb})$ or incorrect reference values $(\mathrm{Rb}$ and Fe). The limit of detection was determined for all measured elements with a multi-point external calibration curve. Using FGS 1 as a calibration check, good bias $(<15 \%)$ was observed for those elements that were above the LOD. 
A description of the casework database provided by the BKA was also given. Compared to the FIU database, the BKA database shows greater variation for each element measured. This is likely due to the fact that the BKA database includes many different types of glass samples, whereas the FIU database includes only float glass obtained from vehicle windshields. Both databases showed a high correlation between $\mathrm{Zr}$ and $\mathrm{Hf}$, suggesting that $\mathrm{Hf}$ (which typically has a much lower concentration than $\mathrm{Zr}$ ) may not be useful for discrimination purposes.

Finally, a brief description of smaller datasets shared by the BKA was also provided. These collections will be useful as test sets in order to evaluate the performance of a likelihood ratio approach to evidence interpretation, the results of which are discussed in the next chapter. 


\section{ANALYSIS AND INTERPRETATION OF LA-ICP-MS GLASS DATA}

Once the glass samples are analyzed and each isotope quantified (Chapter 3), an important consideration is the analysis and interpretation of the collected data. Typically, in forensic casework, a match criterion (e.g., t-test, range overlap, $n$-sigma) is used to compare the known sample to the questioned sample. ${ }^{36-38}$ This approach to evidence interpretation is termed the "frequentist" approach. In the United States, the match criterion outlined in the standard ASTM E2927 is applied for the comparison of the known and questioned sample. ${ }^{115}$ An alternative to the frequentist approach is the "Bayesian" approach, which provides a quantitative measure (the likelihood ratio) of the evidence. In this chapter, both approaches are evaluated and compared.

\subsection{Performance of ASTM E2927 Comparison Criterion}

\subsubsection{Description of ASTM E2927 Comparison Criterion}

ASTM E2927 suggests a modified \pm 4 standard deviations for the comparison between a known $(\mathrm{K})$ and questioned $(\mathrm{Q})$ sample. ${ }^{115}$ First, the average and standard deviation for the $\mathrm{K}$ are calculated. If the calculated standard deviation is less than $3 \%$ of the mean, then $3 \%$ of the mean is used instead of the standard deviation. The comparison interval for the $\mathrm{K}$ is then defined as the average \pm 4 times the standard deviation (or \pm 4 times $3 \%$ of the average, whichever is greatest). The average of the Q sample is then calculated and compared to the $\mathrm{K}$ comparison interval. This procedure is done for all 17 elements measured. If the average of the Q sample lies within the K comparison interval for all 17 elements, then the $\mathrm{K}$ and $\mathrm{Q}$ are indistinguishable. Alternatively, if the average of the $\mathrm{Q}$ 
sample lies outside the $\mathrm{K}$ comparison interval for one or more element(s), then the $\mathrm{K}$ and $\mathrm{Q}$ are distinguishable.

\subsubsection{Comparison Criterion Results for FIU Vehicle Database}

The ASTM comparison criterion, as described above, was applied to all pairwise comparisons for the FIU vehicle database. Since this comparison criterion is asymmetrical, different results may be obtained depending on which sample is treated as the known or questioned sample. Thus, each pair of samples was compared twice using the comparison criterion. For example, Sample 1 (as the known) was compared to Sample 2 (as the questioned) using all 15 replicates of each sample; then the roles were reversed (Sample 1 as the questioned and Sample 2 as the known). This brings the total number of pairwise comparisons to:

$$
n \times(n-1)
$$

In the equation above, $n$ is the number of samples in the database. For the FIU database, $n$ $=420$ and the number of pairwise comparisons is therefore equal to 175,980 . However, in some cases, the inner and outer pane of a vehicle windshield is expected to originate from the same manufacturing source. To account for this, any comparison between the inner and outer pane of the same vehicle was removed, bringing the total number of comparisons down to $175,560(175,980-420)$. This ensures that only comparisons between different vehicles are treated as different sources.

Table 29 shows the false inclusion rate for the FIU database. Only 208 pairs out of the 175,560 totals pairs were associated, giving a low false inclusion rate of $0.12 \%$. Of those 208 false inclusions, 165 were comparisons between vehicles of the same make and/or 
year of manufacture. This may explain why these pairs have similar elemental profiles and were found to be indistinguishable using the ASTM comparison criterion. Unfortunately, without the windshield sticker, the glass manufacturing plant where the windshield glass was produced is unknown. Therefore, it cannot be determined whether these false inclusions are truly random matches or correct associations. Still, even if all false inclusions truly originated from different sources (i.e., different glass manufacturers), the false inclusion rate is quite low.

Table 29 - False inclusion and exclusion for FIU database and duplicates using ASTM comparison criterion

\begin{tabular}{cc} 
& FIU Vehicle Database \\
& ASTM Comparison Criterion \\
\hline False Inclusions & $\mathbf{0 . 1 2 \%}$ \\
& $(208 / 175,560)$ \\
False Exclusions & $\mathbf{5 2 . 5 \%}$ \\
& $(42 / 80)$ \\
\hline
\end{tabular}

In order to assess the false exclusion rate, the 40 duplicates that were analyzed were compared to their respective original sample. That is, Sample 1 was compared to Sample 1 Duplicate, Sample 2 to Sample 2 Duplicate, and so on. As mentioned previously, to account for the asymmetry of the ASTM comparison criterion, each pair was compared twice so that each sample was treated as the known and questioned. Thus, the total number of comparisons is equal to $80(40 \times 2)$. The ASTM criterion led to an extremely high false exclusion rate of $52.5 \%$. The most discriminating elements were $\mathrm{Al}, \mathrm{Zr}$, and $\mathrm{Pb}$. Al was especially problematic for duplicates analyzed on Day 44, which is not surprising since it is suspected that Day 44 suffered from a pulse/analog calibration issue, 
as discussed in Chapter 3. Some of the discriminating elements (e.g., La, Nd, Hf, $\mathrm{Pb}$ ) are present at low concentrations and therefore may have had detection issues. Other elements may have had high inter-day variation that was not accounted for using the ASTM criterion. For example, the BKA found that $\mathrm{Zr}$ and $\mathrm{Hf}$ have high inter-day variation. In order to account for this, the BKA established a fixed relative standard deviation (FRSD) that differs for each element. ${ }^{37}$ Another graduate student in the group, Tricia Hoffman, implemented an approach similar to that of the BKA; the results showed an improved false exclusion rate (1.9\%, compared to $52.5 \%$ for the ASTM criterion).

High inter-day variation might not affect casework, since the known and questioned samples are typically analyzed on the same day, as recommended in ASTM E2927. However, it would affect the use of a database to calculate a frequency of occurrence. For this approach, the questioned sample is compared not only to the known but also to an entire glass database using the ASTM comparison criterion. Then, the number of "matches" is counted (i.e., the number of times the questioned sample is indistinguishable to a sample in the database). The frequency of occurrence is subsequently calculated by:

$$
\text { Frequency }=\frac{M}{N}
$$

$M$ is the number of "matches" and $N$ is the total number of samples in the database (including the known). With the ASTM criterion, it is expected that the frequency of occurrence will be underrepresented. To correct this, a match criterion that accounts for the inter-day variation should be used. For this reason, if a laboratory aims to use a frequency of occurrence approach, the BKA approach is suggested (after the lab establishes its own FRSD). 


\subsubsection{Comparison Criterion Results for BKA Casework Database}

The ASTM criterion was also used to determine the false exclusion rate for the BKA casework database. For the BKA database, $n=385$ and the number of comparison pairs is equal to 147,840 . A false exclusion rate of $0.018 \%(28 / 147,840$ pairs $)$ was obtained. The much lower false exclusion rate for the BKA database, compared to the FIU database, is unsurprising because the FIU database contains samples from similar sources (i.e., same vehicle make/model or year of manufacture), whereas the BKA database includes casework samples of many different glass types.

The false inclusion rate could not be estimated for the BKA database since no duplicate samples were analyzed.

\subsection{Frequentist versus Bayesian Approach}

The frequentist approach has several disadvantages: it suffers from the "fall-off-the-cliff" effect, in which a small change in the evidence can lead to a drastic change in the final decision; it does not account for the rarity of an elemental profile; and it does not provide a weight of evidence. The latter disadvantage, however, may be overcome through the use of a verbal scale in order to assign the strength of an association. Nonetheless, the use

of a verbal scale can be subjective since it relies on the analyst's personal experience. A more objective approach is possible if a database is available. This allows a frequency of occurrence to be calculated by comparing the elemental profile of the questioned sample to all the samples in the database that are known to originate from a different source. Although this calculation would account for the rarity of the elemental profile, it would 
still not address the strict cutoff established by match criteria that leads to the "fall-offthe-cliff" effect. ${ }^{41}$

An alternative to a match criterion is the continuous likelihood ratio (LR), also known as Bayes' factor, which is given by the middle term in Bayes theorem:

$$
\frac{P\left(H_{1} \mid E\right)}{P\left(H_{2} \mid E\right)}=\frac{P\left(E \mid H_{1}\right)}{P\left(E \mid H_{2}\right)} \times \frac{P\left(H_{1}\right)}{P\left(H_{2}\right)}
$$

The first term in Bayes theorem is known as the "posterior odds" and the last term is known as the "prior odds." Each juror determines his own prior odds and it is the role of the LR to update those prior odds to ultimately obtain the posterior odds. However, the forensic analyst is typically only interested in the likelihood ratio, while the prior and posterior odds are reserved for the courts. The LR is defined as the ratio between the probability of the evidence given $\mathrm{H}_{1}$ (an association) and the probability of the evidence given $\mathrm{H}_{2}$ (a non-association). If the $\mathrm{LR}$ is greater than 1, it shows support for the hypothesis of an association $\left(H_{l}\right)$ and if the LR is less than 1, it shows support for the hypothesis of no association $\left(H_{2}\right)$. Furthermore, the larger the LR the stronger the support for $\mathrm{H}_{1}$, while the smaller the LR the stronger the support for $\mathrm{H}_{2}$. A likelihood ratio equal to 1 indicates that neither hypothesis is favored. The LR takes into account the similarity between the known and questioned samples, the within-source and between-source variation, the rarity of the elemental profile, and the correlation between variables. ${ }^{42}$ Moreover, the LR approach answers the post-data question "how much does the evidence...increase the probability that it was the accused who left it," which is what the court is interested in, rather than the pre-data question "what is the probability of obtaining this match by carrying out this procedure," which is what the frequentist 
approach answers. ${ }^{41}$ For this project, the multivariate kernel (MVK) model proposed by Aitken and Lucy was employed for the calculation of the likelihood ratio followed by a post-hoc calibration. ${ }^{40,44-45,140,142}$ Both procedures are discussed in more detail below.

\subsubsection{Multivariate Kernel (MVK) Model}

The MVK model is also referred to as the two-level model since it accounts for two levels of variation: the within-source and between-source variation. The model uses a normal distribution for the within-source variation and a kernel density estimate for the between-source variation. A detailed description of the calculation for the numerator and denominator of the likelihood ratio using this model is given in Aitken and Lucy. ${ }^{40}$ The calculation can be made using the freely available R package "comparison," which requires the additional packages "nnls" and "isotone." Unfortunately, the model leads to an unreasonable weight of evidence for LA-ICP-MS glass data; that is, the model results in extremely large or small LRs. This is likely due to the high dimensionality of the data (i.e., many variables). Thus, to improve the accuracy of the likelihood ratio, a calibration step is necessary.

\subsubsection{Calibration of Likelihood Ratio}

Vergeer, et al. and van Es, et al. reported one method of calibration that involves the use of density models followed by the empirical lower and upper bound (ELUB) method to limit the LR output. ${ }^{44-45}$ This approach to calibration will subsequently be referred to as the "ELUB" method. The authors successfully applied the ELUB method to LRs calculated using the MVK model (also referred to as the two-level model). A casework 
LA-ICP-MS glass database collected at the Netherlands Forensic Institute (NFI) was utilized for all calculations. Low false exclusion $(0.3 \%)$ and false inclusion $(0.2 \%)$ rates were reported. Moreover, the false exclusions and inclusions generally showed weak support for the incorrect hypothesis.

Another method of calibration employs the Pool Adjacent Violators (PAV) algorithm. The PAV transformation improves the calibration of a set of posterior probabilities and sets an upper and lower limit to the LR, while still maintaining the discriminating power of the LRs. ${ }^{140,142}$ A detailed description of the algorithm is given in Appendix C of Zadora, et al. ${ }^{42}$ The PAV transformation has previously been applied to speaker recognition data and SEM-EDX glass data. ${ }^{139-141}$ However, this study presents the first use of the PAV algorithm to calibrate LRs generated for LA-ICP-MS glass data. This calibration approach will subsequently be referred to as the "PAV method."

The performance of the likelihood ratio can be evaluated through the use of Empirical Cross Entropy (ECE) plots, which are described in the book by Zadora, et al. ${ }^{42}$ Moreover, supplementary information for the book (the relevant $\mathrm{R}$ codes and data) is freely available for download from wiley.com. Examples of ECE plots are given in Figure 51. The solid red line represents the ECE for the LR values, the dashed blue line represents the ECE after the PAV transformation, and the dotted grey line represents the ECE for the neutral case in which the LR is always equal to 1 (i.e., the LR provides equal weight for either hypothesis). The lower the solid red curve, the better the accuracy and the lower the dashed blue curve, the better the discrimination. Finally, the difference between the solid and dashed lines is an indication of the calibration; the smaller this difference, the better the calibration. The ECE is plotted against a wide range of prior odds, shown 
on the $\mathrm{x}$-axis. This is important since the forensic analyst does not know the prior odds (this is for the jury to decide). Thus, the LR should perform well over as large a range as possible. That is, the solid line (and dashed line) should fall below the neutral (dotted grey) line for a wide range of prior odds. ${ }^{42,150}$

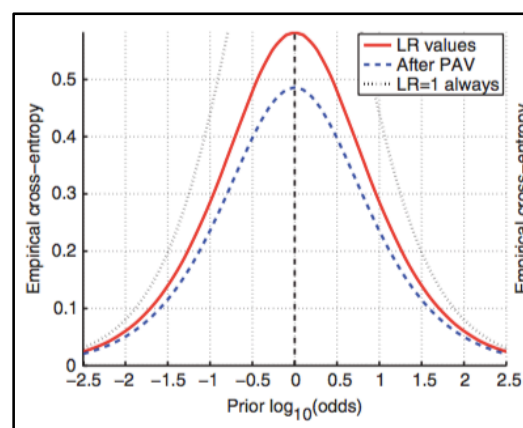

(a)

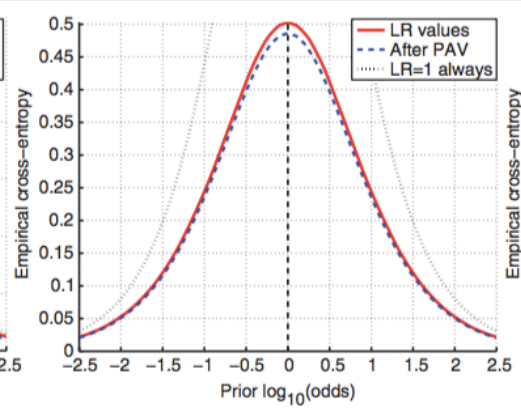

(b)

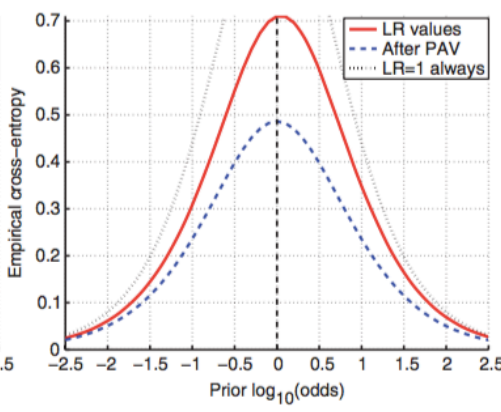

(c)

Figure 51 - Examples of ECE plots in which the validation set of LRs is: (a.) well calibrated, (b.) better calibrated, and (c.) badly calibrated (Zadora, et al. ${ }^{42}$, figure reproduced with permission from Wiley \& Sons, Ltd)

\subsection{Performance of Calibrated Likelihood Ratio for FIU and BKA Database}

\subsubsection{Double 10-Fold Cross Validation using the MVK + PAV Approach}

Prior to the likelihood ratio calculation, all elements were normalized to the element with the highest average concentration (calcium). Then the base-10 logarithm was taken for the 16 element ratios. This data pre-treatment reduces the dimensionality and makes the multivariate probability distribution more amenable in subsequent calculations. A double 10-fold cross validation using the MVK model and PAV calibration was implemented in Matlab by Javier Franco-Pedroso and Daniel Ramos using the databases collected at FIU and the BKA; the results of this study have been published in Talanta. ${ }^{46}$ Each database was split into 10 groups and the calibrated LRs were computed for all pairwise 
comparisons within one group while the remaining 9 groups were used as the background population for the MVK model. The procedure was then repeated for each of the 10 groups (this iteration will be referred to as the "outer loop"). Within the 9 groups used as the background population, a second 10 -fold cross validation was implemented to train the PAV model. The 9 groups were pooled together and then split into 10 groups. The uncalibrated LRs were calculated for all pairwise comparisons within one group, while the remaining 9 groups served as the background population for the MVK model. Once the un-calibrated LRs were computed for all comparisons in each of the 10 groups (the "inner loop"), the LRs were pooled together and used to train the PAV model, which was then used to calibrate the LRs from the outer loop. Figure 52 shows a schematic of the double 10-fold cross validation procedure.

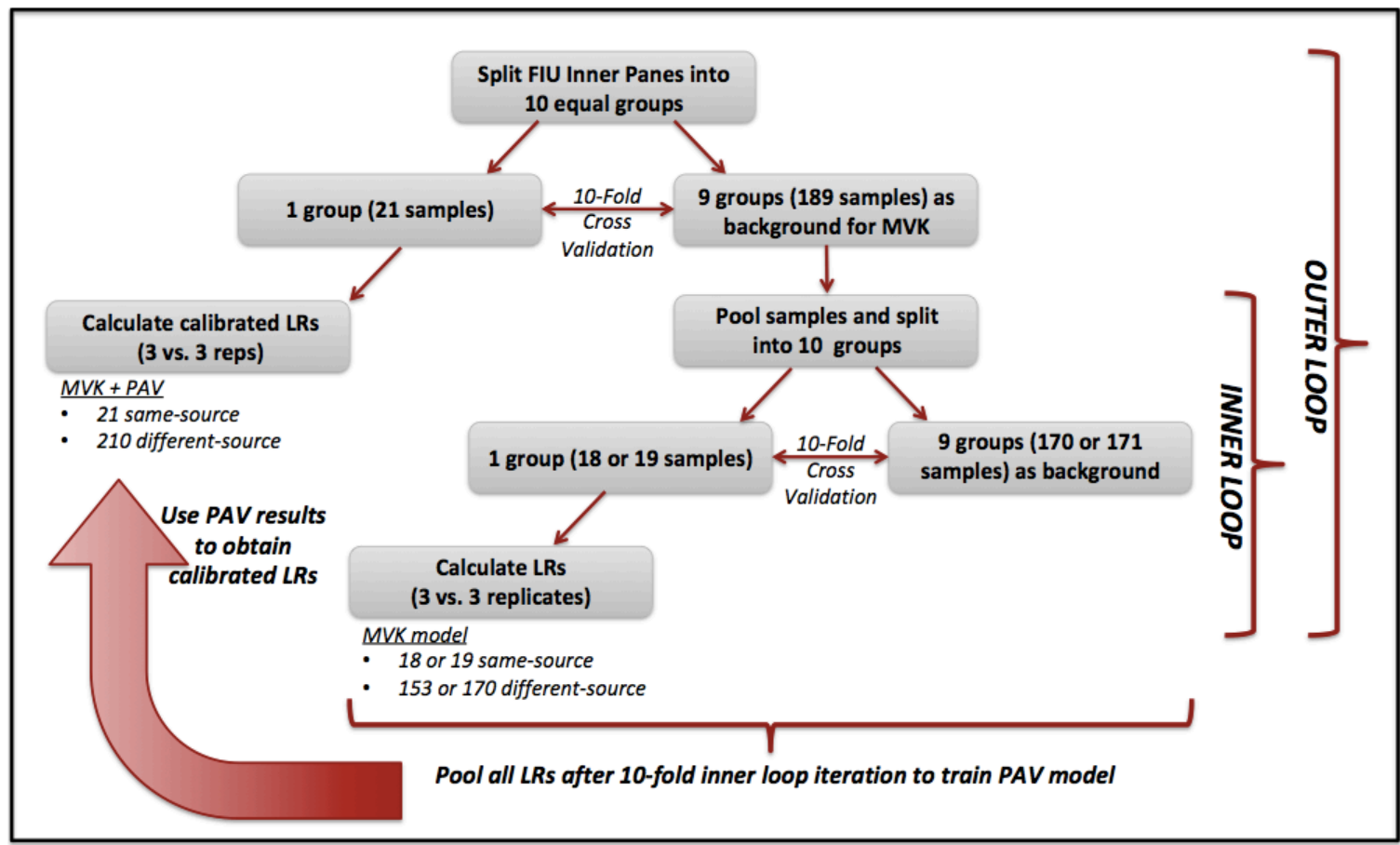

Figure 52 - Schematic of double 10-fold calibration procedure using FIU Inner Panes as an example 
The false exclusion rate can be estimated using pairwise comparisons known to originate from the same source. Likewise, the false inclusion rate can be estimated with pairwise comparisons known to originate from different sources. Same-source pairwise comparisons were generated by comparing the first 3 replicates of each sample to the last 3 replicates of the same sample. Although 15 replicate measurements were collected for the FIU database, only 6 replicates were used in order to match the number of replicates in the BKA database; this was especially important in the case where the BKA database was used as the background database to compute LRs for the FIU database (discussed below). The likelihood ratio is symmetrical, so the total number of same-source comparisons is simply given by the size of each database (385 and 420 for the BKA and FIU database, respectively). This approach of spitting the replicates of a sample in order to estimate the false exclusion rate is not ideal since inter-day variation is not accounted for. It would be better to obtain duplicates (analyzed on a different day than the original) for each sample. However, it would be time-consuming to reanalyze every sample in the database. Since a large number of same-source pairwise comparisons are necessary to calibrate the likelihood ratio, the current method of splitting replicates is the most practical solution.

Different-source pairwise comparisons were obtained by comparing the first 3 replicates of each sample to the last 3 replicates of all different samples within each of the 10 groups of the outer loop. Since the BKA database is not divisible by 10,5 groups contained 38 samples and 5 groups contained 39 samples. Thus, the total number of different-source comparison pairs is given by: 


$$
5 \times \frac{(38 \times(38-1))}{2}+5 \times \frac{(39 \times(39-1))}{2}=7220
$$

For the FIU database, "different-source" refers to panes that originated from different vehicles. This is because, in some cases, the inner and outer panes of a vehicle windshield originate from the same source. Therefore, the FIU database was first separated into inner and outer panes and the 10-fold cross validation procedure was done separately for the inner panes and for the outer panes. The total number of different-source pairs is then 4,200 (2,100 for the inner panes plus 2,100 for the outer panes):

$$
2 \times \frac{\left(\frac{210}{10}\right)\left(\frac{210}{10}-1\right)}{2} \times 10=4200
$$

A second approach for the FIU database was also completed. Rather than using the FIU database as both the training and test set via a double cross validation procedure, the BKA database was used as the background population for the MVK model and to train the PAV algorithm via a single 10 -fold cross. Once the MVK and PAV models were trained with the BKA database, calibrated LRs for the FIU database were calculated. In this case, the number of different-source comparison pairs for the FIU database is given by all possible combinations between the inner panes plus all possible combinations between the outer panes, giving a total of 43,890 pairs:

$$
2 \times \frac{(210 \times(210-1))}{2}=43890
$$

Using the BKA database as the background population to calculate LRs for the FIU database is preferable since the BKA database includes real-world samples from actual cases submitted to the laboratory. On the other hand, the FIU database is comprised of glass from vehicles manufactured within a narrow time frame, many of which have the 
same make and/or year of manufacture. The BKA database therefore provides a more relevant population than the limited FIU database. Additionally, using the same database to train both the MVK and PAV models via a double cross validation may lead to overly optimistic results.

As mentioned previously, the MVK model tends to lead to unreasonably large (or small) likelihood ratios for LA-ICP-MS glass data. Most of the same-source pairs had an LR greater than $10^{20}$, while most of the different-source pairs had an LR less than $10^{-300}$. The top left panel in Figure 53 shows the un-calibrated $\log 10$-likelihood ratios for samesource (in blue) and different-source (in red) pairs for the BKA database. The little overlap between the two histograms is an indication of the excellent discrimination capabilities of LA-ICP-MS, but the extreme LR values indicate poor calibration. The Pool Adjacent Violators (PAV) Algorithm not only calibrates the LRs, but also sets an upper and lower bound to the likelihood ratio. The top right panel in Figure 53 shows the $\log 10$-likelihood ratios for the double 10-fold BKA database after the PAV transformation. Excellent discrimination is still retained, while the LR is limited to a range of about $10^{-2.5}-10^{3.8}$ (or $3.0 \times 10^{-3}$ to $6.1 \times 10^{3}$ ). Similar results were obtained for the double 10-fold FIU database (middle panels in Figure 53) and the FIU database using the BKA database as the background population (bottom panels in Figure 53).

Figure 54 shows the ECE plots before (the three left panels) and after (the three right panels) calibration for the double 10-fold BKA database (top), double 10-fold FIU database (middle), and FIU database with the BKA database as the background (bottom). In all cases, it is evident that the un-calibrated LRs perform worse than the neutral case $(\mathrm{LR}=1)$; that is, the solid red line lies above the dotted grey line. Additionally, the 
accuracy for all three cases is very poor (accuracy is given by the height of the red line). On the other hand, the discrimination (given by the height of the blue line) is good. The large gap between the red and blue lines indicates very poor calibration. After calibration using the PAV algorithm, the results significantly improve. For all three cases, the red line (accuracy) is below the neutral dotted grey line for a wide range of prior odds and the blue line shows good discrimination.

Additionally, the gap between the red and blue line is quite small (note the scale of the yaxis), indicating good calibration. Several useful metrics can be obtained from the ECE plots in order to gauge the performance of the LRs. The cost log-likelihood ratio (Cllr), which is a measure of accuracy, is the ECE value where the red line crosses a $\log 10$ prior odds equal to zero $(\mathrm{x}=0)$. The closer the Cllr is to zero, the better the accuracy. The minimum cost log-likelihood ratio $\left(\mathrm{Cllr}_{\min }\right)$, which is a measure of discrimination, is the ECE value where the blue line crosses a $\log 10$ prior odds equal to zero. The closer the Cllr is to zero, the better the discrimination. Finally, the calibration cost log-likelihood ratio $\left(\mathrm{Cllr}_{\text {cal }}\right)$ is the difference between $\mathrm{Cllr}$ and $\mathrm{Cllr}_{\min }$; the smaller the difference, the better the calibration. These three metrics are useful for the relative comparison of different LR systems. 

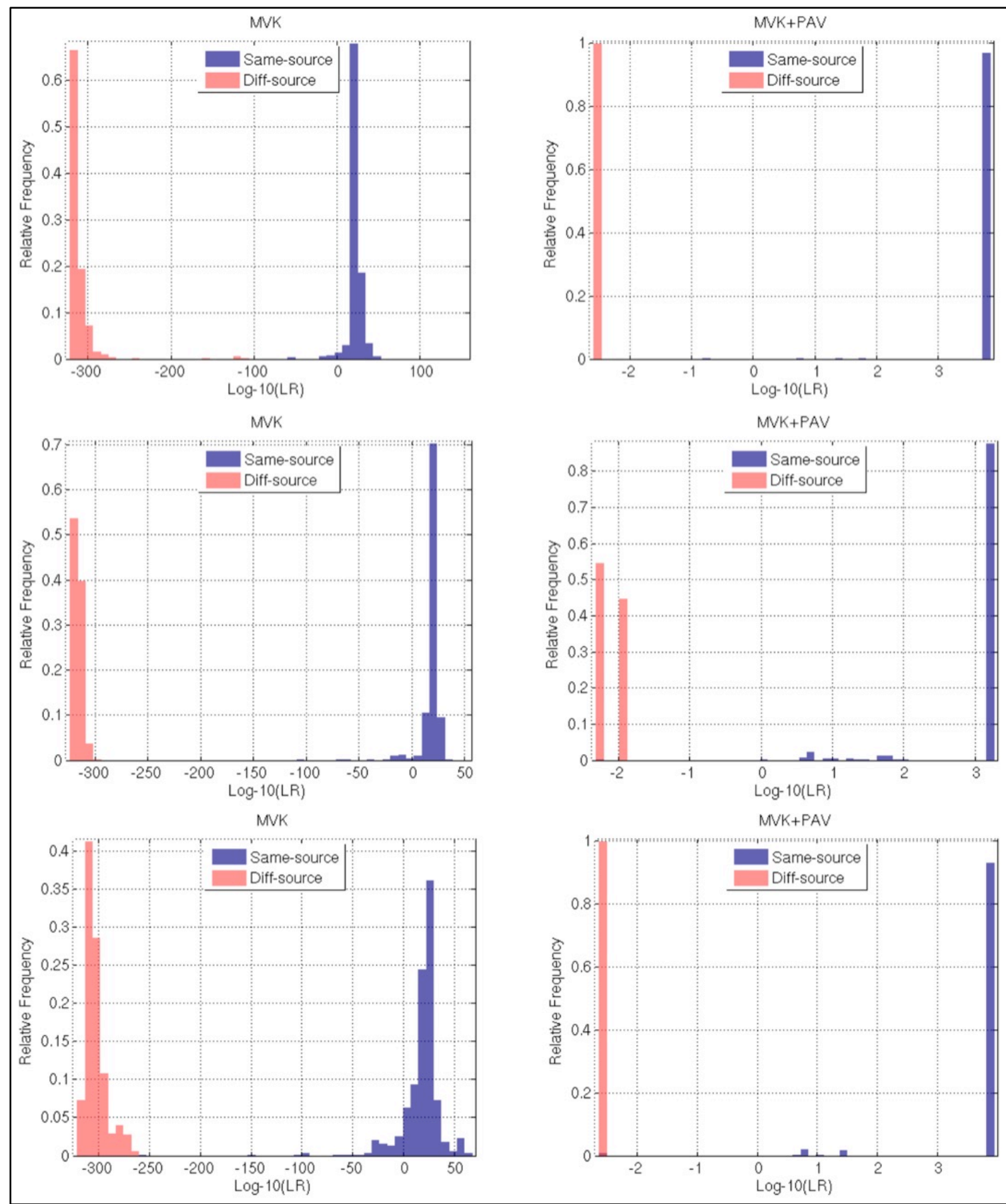

Figure 53 - Un-calibrated (left panels) and calibrated (right panels) $\log 10$ LRs for the BKA database (top), the FIU database (middle), and the FIU database with the BKA database as the background population (bottom). 


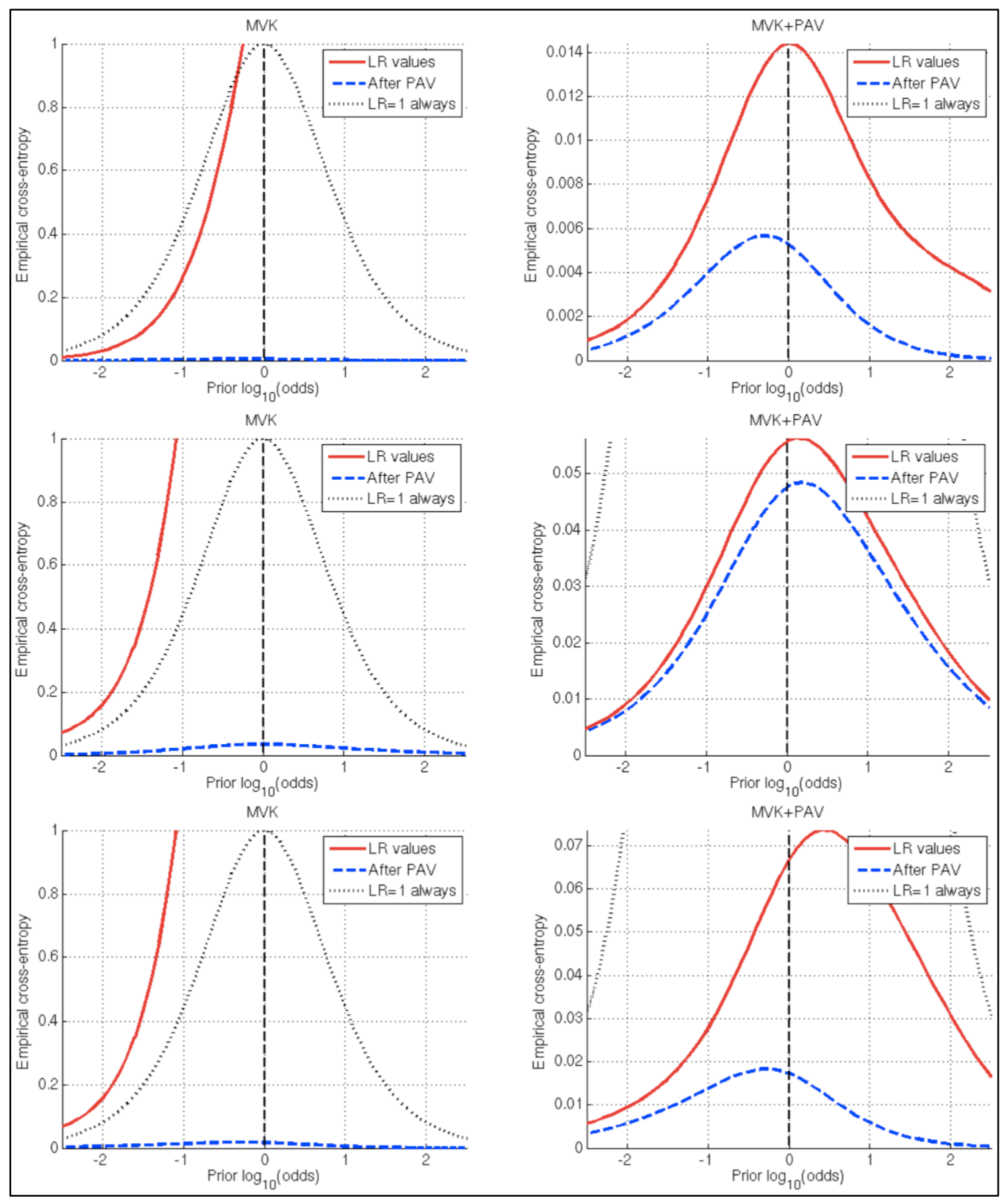

Figure 54 - Un-calibrated (left panels) and calibrated (right panels) ECE plots for the BKA database (top), the FIU database (middle), and the FIU database with the BKA database as the background population (bottom). 
Table 30 - False exclusion rate, false inclusion rate, Cllr and Cllr $r_{\text {min }}$ (if applicable) for the BKA and FIU databases using different comparison criteria. Fractions within parentheses indicate the number of pairs that were falsely excluded/included over the total number of pairwise comparisons.

\begin{tabular}{cccccc}
\hline & \multicolumn{2}{c}{ BKA Database } & \multicolumn{3}{c}{ FIU Database } \\
\cline { 2 - 6 } & $\begin{array}{c}\text { ASTM } \\
\text { Criterion }\end{array}$ & $\begin{array}{c}\text { LR } \\
(\mathbf{1 0 - f o l d})\end{array}$ & $\begin{array}{c}\text { ASTM } \\
\text { Criterion }\end{array}$ & $\begin{array}{c}\text { LR } \\
(\mathbf{1 0 - f o l d )}\end{array}$ & $\begin{array}{c}\text { LR } \\
\text { (with BKA) }\end{array}$ \\
\hline \% False & $\mathbf{3 . 2 5 \%}$ & $\mathbf{0 . 5 2 \%}$ & $\mathbf{7 . 5 0 \%}$ & $\mathbf{1 . 1 9 \%}$ & $\mathbf{1 . 1 9 \%}$ \\
Exclusions & $(25 / 770)$ & $(2 / 385)$ & $(63 / 840)$ & $(5 / 420)$ & $(5 / 420)$ \\
\% False & $\mathbf{0 . 0 2 2 \%}$ & $\mathbf{0 . 2 1 \%}$ & $\mathbf{0 . 1 0 \%}$ & $\mathbf{0 . 6 0 \%}$ & $\mathbf{0 . 3 3 \%}$ \\
Inclusions & $(33 / 147840)$ & $(15 / 7220)$ & $(88 / 87780)$ & $(25 / 4200)$ & $(143 / 43890)$ \\
Cllr & - & 0.014 & - & 0.056 & 0.067 \\
Cllr $_{\text {min }}$ & - & 0.005 & - & 0.048 & 0.017 \\
Cllr $_{\text {cal }}$ & - & 0.009 & - & 0.008 & 0.049 \\
\hline
\end{tabular}

Table 30 shows the error rates for the FIU and BKA database using both the calibrated likelihood ratio and the ASTM comparison criterion. In order to fairly compare the two approaches, the pairwise comparisons using the ASTM criterion were redone so that the first 3 replicates of one sample were compared to the last 3 replicates of the same sample (for same-source comparisons) or a different sample (for different-source comparisons). Additionally, for the FIU database, all comparisons between inner and outer panes were excluded. Note that the total number of pairwise comparisons for the ATSM criterion is double that of the likelihood ratio; this is because the likelihood ratio is symmetrical, while the ASTM criterion is not (Chapter 3).

Compared to the calibrated likelihood ratio, the ASTM criterion led to a higher false exclusion rate: $3.25 \%$ for the BKA database and $7.50 \%$ for the FIU database. It should be noted, however, that ASTM E2927 suggests a minimum of 9 replicate measurements to fully characterize the known sample, but only 3 replicate measurements were used in this study. It is possible that the high false exclusion rate is due to insufficient replicate 
measurements to fully characterize the known sample. The calibrated likelihood ratio offered a lower false exclusion rate: $0.52 \%$ for the BKA database and $1.19 \%$ for the FIU database (regardless of the background database used).

On the other hand, the false inclusion rate was lower for the ASTM criterion than the calibrated likelihood ratio. For the BKA database, a total of 31 pairs were associated using the ASTM criterion, leading to a low false inclusion rate of $0.022 \%$. Using the calibrated LR, 15 pairs were associated yielding a false inclusion rate that was an order of magnitude greater than that of the ASTM criterion. Nonetheless, in both cases, the false inclusion rate is quite low $(<0.5 \%)$. Unfortunately, since the samples in the BKA database are casework samples, their origin is unknown. Therefore, it cannot be stated whether the two samples being compared were manufactured in the same glass plant at around the same time or whether, despite originating from different sources, the two samples have a chemical profile that coincidentally "match."

For the FIU database, the ASTM criterion resulted in a low false inclusion rate of $0.10 \%$. However, though higher, the calibrated LR still performed well $(<1 \%)$ regardless of which database was used as the background population.

Apart from the rates of misleading evidence (false exclusions and inclusions), other metrics to assess the performance of the likelihood ratio include the previously mentioned log-likelihood ratio cost $(\mathrm{Cllr})$ and the minimum log-likelihood ratio cost $\left(\mathrm{Cllr}_{\mathrm{min}}\right){ }^{150}$ Table 30 shows a Cllr of 0.014 and a Cllr $r_{\min }$ of 0.049 (both values close to zero) for the BKA database, indicating both good accuracy and discrimination. Slightly worse accuracy and discrimination is seen for the two FIU database approaches. However, the Cllr and the Cllr $r_{\text {min }}$ are still fairly close to zero for both cases. The calibration cost log- 
likelihood ratio $\left(\mathrm{Cllr}_{\text {cal }}\right)$ can be calculated by subtracting the $\mathrm{Cllr}_{\min }$ from the Cllr. The Cllr $r_{\text {cal }}$ values are $0.008,0.009$, and 0.05 for the double 10 -fold FIU database, the double 10-fold BKA database, and the FIU database with the BKA database as the background, respectively. Thus, the double 10-fold BKA and FIU database are approximately equally well calibrated (though the BKA database has better accuracy and discrimination). The FIU database with BKA background is well calibrated, but not as well as the other two approaches.

Table 31 - Percent of falsely inclusions that originate from the same vehicle make and/or year of manufacture for the FIU database. The fractions within parentheses indicate the number of false inclusions over the total number of different-source pairwise comparisons.

\begin{tabular}{cccc}
\hline & ASTM Criterion & LR (10-fold) & LR (with BKA) \\
\hline Same Vehicle Make and & $\mathbf{0 . 7 9 \%}$ & $\mathbf{0 . 4 8 \%}$ & $\mathbf{0 . 1 2 \%}$ \\
Year & $(57 / 7220)$ & $(20 / 4200)$ & $(53 / 43890)$ \\
Same Vehicle Make, & $\mathbf{0 . 0 1 4 \%}$ & $\mathbf{0 . 0 4 8 \%}$ & $\mathbf{0 . 0 2 7 \%}$ \\
Different Year & $(1 / 7220)$ & $(2 / 4200)$ & $(12 / 43890)$ \\
Same Year, Different & $\mathbf{0 . 2 2 \%}$ & $\mathbf{0 . 0 2 4 \%}$ & $\mathbf{0 . 0 7 1 \%}$ \\
Vehicle Make & $(16 / 7220)$ & $(1 / 4200)$ & $(31 / 43890)$ \\
Different Vehicle Make & $\mathbf{0 . 1 9 \%}$ & $\mathbf{0 . 0 4 8 \%}$ & $\mathbf{0 . 1 2 \%}$ \\
and Year & $(14 / 7220)$ & $(2 / 4200)$ & $(47 / 43890)$ \\
\hline
\end{tabular}

Since all of the FIU glass samples originated from known vehicles, it is possible to determine whether the glass from two different vehicles may have been produced in the same glass manufacturing plant. Table 31 shows the percentage of falsely included pairs that originated from similar vehicles. The greatest percentage of falsely included pairs originated from vehicles that have the same make and year of manufacture, thus it is likely that the windshield glass for these vehicles were produced in the same glass manufacturing plant and are therefore indistinguishable. In some cases, different 
automobile manufacturers obtain their glass from the same glass manufacturer; this may account for the falsely included pairs that have a different make but same year of manufacture. Relatively few false inclusions had the same vehicle make but different year of manufacture; for the most part, these vehicles were manufactured 1 to 2 years apart. The remaining pairs had a different vehicle make and year of manufacture; these too were typically manufactured 1 to 2 years apart. The highest number of false inclusions that originated from a different vehicle make and different year of manufacture can be seen for the calibrated LR using the BKA database as the background population. These pairs may genuinely be coincidental "matches" or, despite having different vehicle manufacturers, may have the same glass manufacturer. Unfortunately, although the vehicle origin for each glass sample is known, the windshield sticker that discloses the glass manufacturing plant was absent or illegible for most vehicles. Without this sticker, it is unknown whether the same glass manufacturer produced the windshield glass for these falsely included pairs.

Many of the falsely excluded pairs using the ASTM criterion were discriminated by $\mathrm{Pb}$, $\mathrm{Li}$, and/or Hf, all of which are typically present at low concentrations $(<3 \mathrm{ppm})$ and are therefore near the limit of detection. The remaining false exclusions were distinguished by: $\mathrm{Zr}, \mathrm{Nd}, \mathrm{Sr}, \mathrm{La}, \mathrm{Ce}, \mathrm{Ba}, \mathrm{Rb}, \mathrm{Mn}$, and/or Ti. This may be because the ASTM criterion does not account for large (> 3\% relative standard deviation) inter-day variation. Rather than using a minimum of $3 \%$ of the average, it may be beneficial to establish a different minimum depending on the inter-day variation for each particular element (the approach that the BKA applies for their match criterion). ${ }^{37}$ Some of the false exclusions were due to the "fall-off-the-cliff" effect, in which the average concentration of the questioned 
sample fell just outside the comparison interval for the known sample. For example, one same-source pair was discriminated by $\mathrm{Ba}$ and $\mathrm{Pb}$. For both elements a difference of less than $0.2 \mathrm{ppm}$ for the average concentration of the questioned sample would have led to a correct association. The calibrated likelihood ratio correctly associated most of the falsely excluded pairs that were due to the "fall-off-the-cliff" for the ASTM criterion. The "falloff-the-cliff" effect can also be seen for the false inclusions, in which the average concentration of one or more element(s) of the questioned sample falls just within the known comparison interval. However, for reasons discussed previously, it is uncertain whether the glass from the falsely included pairs was manufactured at the same plant, and therefore has a similar chemical profile.

An advantage of the likelihood ratio over the ASTM criterion is that it provides a continuous approach to evidence interpretation rather than a binary approach (i.e., "match" versus "non-match"). Moreover, the magnitude of the likelihood ratio is an indication of the weight of evidence: the larger the likelihood ratio, the stronger the support for an association, while the smaller the likelihood ratio, the stronger the support for no association. It is expected that the LRs for same-source pairs show strong support for an association (LR $>>1$ ) and different-source pairs show strong support for an exclusion (LR $<<1)$. This is what is observed for both the BKA and FIU database: the majority of the same-source pairs have calibrated LRs at or near the upper limit established by the PAV algorithm and different-source pairs have calibrated LRs at or near the lower limit (Figure 53). It is also expected that falsely included pairs show only weak support for an association, while falsely excluded pairs show only weak support for a non-association. 
Table 32 - Weight of evidence for false inclusions for each database. The fractions within parentheses indicate the number of pairs that fall within a particular likelihood ratio range (given in the leftmost column) over the total number of different-source pairwise comparisons.

\begin{tabular}{|c|c|c|c|c|}
\hline LR Range & $\begin{array}{c}\text { BKA } \\
\text { (10-fold) }\end{array}$ & $\begin{array}{c}\text { FIU } \\
\text { (10-fold) }\end{array}$ & $\begin{array}{c}\text { FIU } \\
\text { (with BKA) }\end{array}$ & $\begin{array}{c}\text { Verbal Scale } \\
\left(\text { Support for } \mathrm{H}_{1}\right)\end{array}$ \\
\hline$>1-10$ & $\begin{array}{c}\mathbf{0 . 1 4 \%} \\
(10 / 7220)\end{array}$ & $\begin{array}{c}\mathbf{0 . 2 1 \%} \\
(9 / 4200)\end{array}$ & $\begin{array}{c}\mathbf{0 . 2 1 \%} \\
(91 / 43890)\end{array}$ & Weak support \\
\hline$>10-100$ & $\begin{array}{c}\mathbf{0 . 0 7 \%} \\
(5 / 7220)\end{array}$ & $\begin{array}{c}\mathbf{0 . 3 1 \%} \\
(13 / 4200)\end{array}$ & $\begin{array}{c}\mathbf{0 . 0 3 4 \%} \\
(15 / 43890)\end{array}$ & Moderate support \\
\hline$>100-1,000$ & 0 & $\begin{array}{l}\mathbf{0 . 0 2 4 \%} \% \\
(1 / 4200)\end{array}$ & 0 & Moderately strong support \\
\hline$>1,000-10,000$ & $\mathbf{0}$ & $\begin{array}{l}\mathbf{0 . 0 4 8 \%} \\
(2 / 4200)\end{array}$ & $\begin{array}{c}\mathbf{0 . 0 8 4 \%} \\
(37 / 43890)\end{array}$ & Strong support \\
\hline
\end{tabular}

Table 32 shows the percentage of falsely included pairs that fall within a particular range of LR values along with the corresponding verbal equivalent. ${ }^{41}$ Most of the falsely included pairs, for both the BKA and FIU database, fall within a LR of $>1-100$, which shows weak to moderate support for the incorrect hypothesis (an association). Using the ASTM criterion, these pairs would simply be said to be associated, without taking the strength of the association into account. Several false inclusions showed strong support of an association for the FIU database using the BKA database as the background population. However, the majority of these pairs (32 out of 37 pairs) originated from vehicles with the same make and/or year of manufacture, which may explain their similar elemental profile. The false exclusions showed similar results: most pairs for both the double 10-fold BKA and FIU databases showed weak to moderate support for $\mathrm{H}_{2}$ (no association). For the FIU database using the BKA database as the background population, all five false exclusions showed moderately strong support for $\mathrm{H}_{2}$. Nevertheless, the false exclusion rate was still low for all cases $(<1.5 \%)$. 


\subsubsection{Double 10-Fold Cross Validation using MVK + PAV Approach with Limited Databases}

After the calibration using Matlab, described in the previous section, was completed, it was decided that Hf should be removed from the element menu. As discussed in Chapter 3, Hf is highly correlated with $\mathrm{Zr}$ and is present at very low concentrations, thus its usefulness for discrimination is limited. Additionally, several samples were removed from the BKA and FIU database. Some samples in the BKA database were found to have the same LR (weak exclusion) when compared to all other samples in the database; this was unusual because the samples in question were found to be extremely different in elemental composition. These weak exclusions for extremely different samples are suspected to have occurred because the BKA database is dominated by float glass samples and only include very few non-float glass samples. To remedy this situation, suspected non-float glass samples were removed from the BKA database, leaving a total of 370 samples. For the FIU database, outer panes that were indistinguishable from the inner pane of the same vehicle were removed, since it is possible that these inner and outer panes originated from the same glass source and are therefore skewing the different-source variation. After removal of these outer panes, the FIU database contained 333 samples. Note that in this case, unlike what was described in the previous section, the inner and outer panes were not treated independently for the LR calculations; that is, the different-source pairs including comparisons between inner versus inner, outer versus outer, and inner versus outer panes. The exclusion of hafnium and several problematic samples were the major driving force to redo the MVK + PAV approach for both the FIU and BKA database. The same data pre-processing was applied: all elements were 
normalized to calcium and the $\log 10$ was taken for the element ratios. The MVK and PAV calculations were implemented using R; the R code for the PAV algorithm was kindly provided by Peter Vergeer of the Netherlands Forensic Institute (NFI).

Table 33 lists the error rates for the BKA database, the FIU database, and the combined $(\mathrm{BKA}+\mathrm{FIU})$ database before and after the exclusion of problematic samples. Note that the error rates for the double 10 -fold cross validation of the full databases (i.e., including the problematic samples) presented in Table 30 do not match those presented in Table 33. This difference is because of the exclusion of hafnium and slight differences in the algorithms used. An improvement in the error rates for the BKA and combined databases can be seen. On the other hand, similar error rates are observed for the FIU database; this may be because the FIU database contains many similar samples (i.e., same make, model, and/or year), even after the exclusion of outer panes that were indistinguishable from the inner pane of the same vehicle. Still, the limited FIU database offered an improvement in the weight of evidence: same-source samples showed stronger support for an association, different-source samples showed stronger support for an exclusion, and misleading evidence showed weaker support for the incorrect hypothesis. Thus, removing problematic samples led to better overall results for all three databases.

Table 33 - Error rates for the BKA, FIU, and combined database before and after the removal of problematic samples

\begin{tabular}{c|cc|cc|cc} 
& BKA & BKA & FIU & FIU & BKA + FIU & BKA + FIU \\
& Full & Limited & Full & Limited & Full & Limited \\
\hline \% False & $\mathbf{1 . 3 0} \%$ & $\mathbf{0 . 5 4 \%}$ & $\mathbf{1 . 4 3 \%}$ & $\mathbf{1 . 5 0 \%}$ & $\mathbf{0 . 6 2 \%}$ & $\mathbf{0 . 1 4 \%}$ \\
Exclusions & $(5 / 385)$ & $(2 / 370)$ & $(6 / 420)$ & $(5 / 333)$ & $(5 / 805)$ & $(1 / 703)$ \\
\% False & $\mathbf{1 . 6 9 \%}$ & $\mathbf{0 . 2 4 \%}$ & $\mathbf{0 . 3 5 \%}$ & $\mathbf{0 . 3 3 \%}$ & $\mathbf{0 . 6 0 \%}$ & $\mathbf{0 . 3 9 \%}$ \\
Inclusions & $(122 / 7220)$ & $(16 / 6660)$ & $(29 / 8400)$ & $(18 / 5379)$ & $(160 / 31793)$ & $(96 / 24360)$ \\
\hline
\end{tabular}




\subsubsection{Double 10-Fold Cross Validation using the MVK + ELUB Approach}

A second calibration procedure, the ELUB approach, was also investigated. ${ }^{44-45}$ The ELUB approach uses a kernel density estimation to calculate the different-source probabilities and a best-fit distribution function to calculate the same-source probabilities. After calibration, upper and lower limits are calculated using a Normalized Bayes Error rate, which takes into account the misleading evidence as well as the number of samesource and different-source comparison pairs. The same procedure described for the PAV method was used for the ELUB method: double 10-fold cross validation, exclusion of hafnium, and normalization to calcium followed by log (base 10) transformation.

After testing several distribution functions (Gaussian, Cauchy, Exponential, Weibull, Gamma, and T Distribution), the scaled T Distribution was selected for the same-source comparisons. Figure 55 shows the fit for the BKA and the FIU database. A QQ plot is also shown to illustrate the goodness of fit. Note that this figure only shows the fit for one of the ten groups in the double 10-fold cross validation; some groups showed a good fit, while others showed a greater deviation from the diagonal in the QQ plot. As can be seen in Figure 55, the majority of the data points lie on the diagonal of the QQ plot, indicating a good fit; however, it is clear that the tails, especially for the BKA database, deviate strongly from the distribution. This is particularly problematic for the left tail since the left side of the same-source histogram coincides with the different-source histogram and as such, will influence the calibrated LR more strongly. The different-source histogram is not included in Figure 55 since the kernel density estimation is fairly straightforward to implement; for more information on the kernel density estimation, the reader may refer to van Es, et al. and Vergeer, et al. ${ }^{44-45}$ 

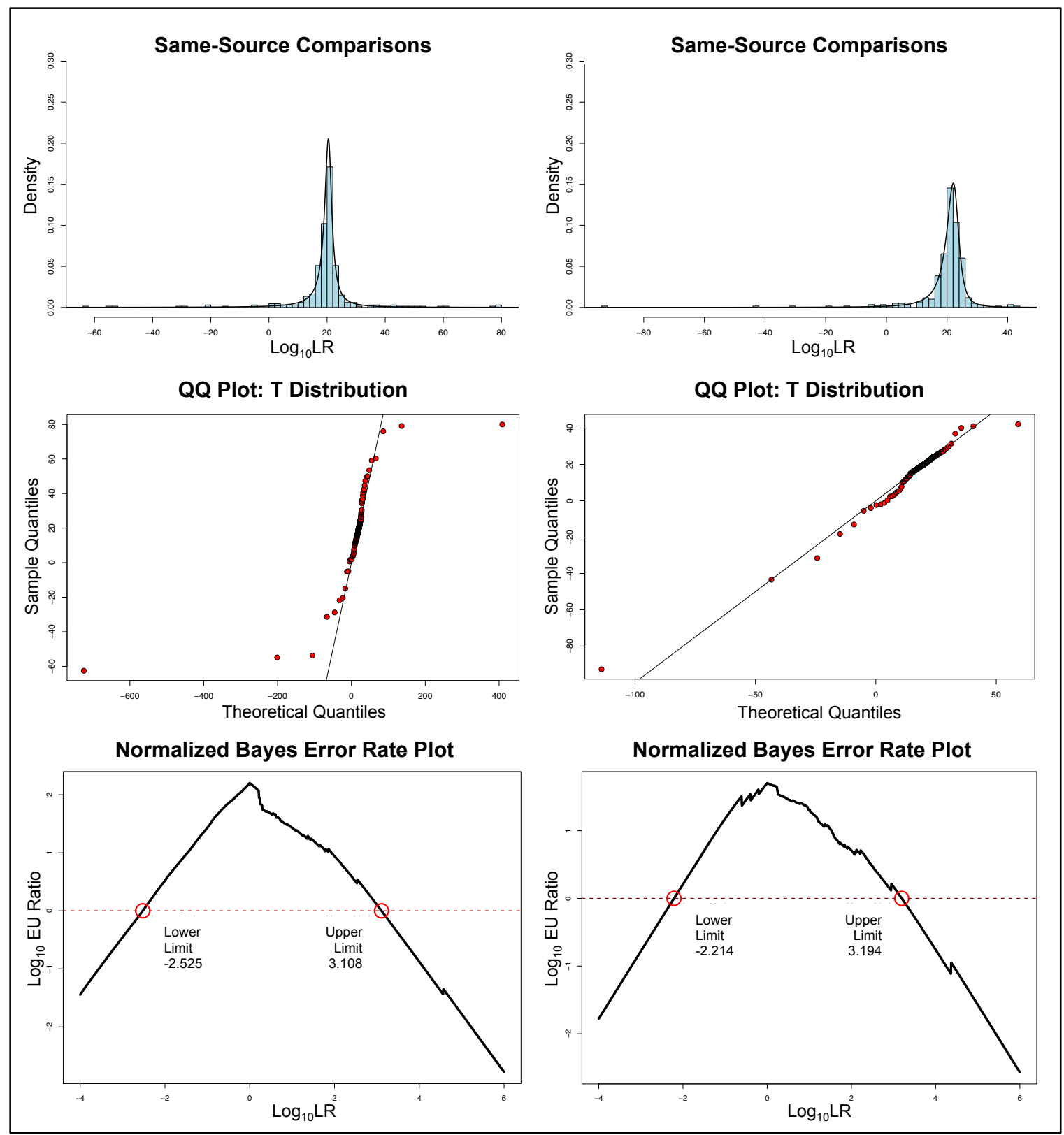

Figure 55 - Scaled T Distribution (top), QQ plot for the Scaled T Distribution (middle), and Normalized Bayes Error rate plot (bottom) for the BKA database (left panels) and the FIU database (right panels)

Figure 55 also shows the Normalized Bayes Error (NBE) rate plot for the calibrated LRs. $^{45}$ The two points where the solid black line crosses $y=0$ indicate the lower and upper limits of the calibrated LR. For example, the lower limit for the BKA database (see 
the bottom left panel in Figure 55) is calculated to be -2.525; note that the axes are on a $\log _{10}$ scale, so this value corresponds to a LR of $0.00299\left(10^{-2.525}\right)$. The upper LR limit for the BKA database is $1,282\left(10^{3.108}\right)$. The FIU database resulted in similar limits: 0.00611 $\left(10^{-2.214}\right)$ to $1,563\left(10^{3.194}\right)$.

Table 34 - Comparison of error rates using the PAV and the ELUB calibration method for the limited FIU and the limited BKA database

\begin{tabular}{ccccc} 
& BKA & BKA & FIU & FIU \\
& ELUB & P AV & ELUB & PAV \\
\hline \% False & $\mathbf{0}$ & $\mathbf{0 . 5 4 \%}$ & $\mathbf{1 . 2 0} \%$ & $\mathbf{1 . 5 0 \%}$ \\
Exclusions & $(0 / 370)$ & $(2 / 370)$ & $(4 / 333)$ & $(5 / 333)$ \\
\% False & $\mathbf{0 . 3 5 \%}$ & $\mathbf{0 . 2 4 \%}$ & $\mathbf{0 . 4 6 \%}$ & $\mathbf{0 . 3 3 \%}$ \\
Inclusions & $(23 / 6660)$ & $(16 / 6660)$ & $(25 / 5379)$ & $(18 / 5379)$ \\
\hline
\end{tabular}

Table 34 compares the error rates of the ELUB approach to those of the PAV approach. The PAV approach led to a slightly higher false exclusion rate and a slightly lower false inclusion rate for both databases. The lower LR limits for both the ELUB and PAV approach were similar. However, the PAV resulted in larger upper limits for both databases: 5,235 versus 1,282 for the BKA database and 3,890 versus 1,563 for the FIU database. Thus, the PAV algorithm provides stronger support for an association. Moreover, the ELUB approach resulted in a greater number of different-source pairs that showed weak $(1<\mathrm{LR} \leq 0.1)$ or moderate $(0.1<\mathrm{LR} \leq 0.01)$ support for an exclusion. As mentioned earlier, the left tail of the same-source distribution greatly influences the calculation of the calibrated LR. Thus, the poor fit at the tails of the same-source histogram likely contributed to the smaller upper LR limit and the high incidence of weak or moderate support for the correct proposition. Unfortunately, the limited database sizes 
makes it difficult to ascertain whether the scaled $\mathrm{T}$ Distribution is indeed the best function to model the same-source distribution. With a larger sample size, the true distribution would be more easily determined. Because of the better overall performance of the PAV calibration method, this approach was selected to calculate a calibrated LR for several small independent datasets. The results are presented in the next section.

\subsection{Performance of Likelihood Ratio for Additional Test Datasets}

This section describes the performance of the calibrated LR for the smaller datasets mentioned in Chapter 3. This allows the performance of the calibrated LR to be assessed for an independent set of data (i.e., a dataset that was not used for the calibration procedure). Since the limited BKA database, using the PAV calibration approach, provided the best results in terms of error rates and weight of evidence, this database was selected as the background population for the smaller independent datasets. Furthermore, the BKA database is more representative of a relevant population as it contains realworld samples that were analyzed as part of casework. The FIU and combined database were not tested for the calculation of the smaller independent datasets; however, Chapter 5 compares each of the three databases for the calculation of a calibrated LR using data collected as part of an inter-laboratory study. A single, rather than a double, 10-fold cross validation was employed using the BKA database. The BKA database was split into 10 equal groups (37 samples in each group) and the un-calibrated LR was calculated for all possible comparisons within one group, while the remaining nine groups were used as the background population for the MVK model. Once the un-calibrated LRs were calculated for all ten groups, all LRs were used for the PAV transformation. Finally, this PAV 
transformation was then used to calculate a calibrated LR for the smaller test sets. A double cross validation was previously used because the same database was used as the training set for the MVK and PAV models and as a test set. In the following examples, the BKA database was used as the training set for the MVK and PAV models via a single cross validation procedure, but an independent test set was utilized to evaluate the performance of the PAV-calibrated LR.

\subsubsection{BKA Same Pane}

As mentioned in Chapter 3, this dataset includes 44 glass samples that originated from the same pane. Thus, all pairwise comparisons should be associated. A total of 946 pairwise comparisons were generated $(44 \times 43 \div 2)$. No pairs were falsely excluded, leading to $100 \%$ correct association. Table 35 shows the distribution of the LR as well as the false inclusion and exclusion rates for all independent datasets tested. For the BKA same pane dataset, the majority $(\sim 87 \%)$ showed strong support for an association. However, 66 pairs showed only weak support for an association. Using the ASTM match criterion, most of these pairs were excluded on the basis of zirconium and/or lead, both of which have known reproducibility issues. Several of the pairs that showed moderate support for an association were discriminated by zirconium and/or lead as well as aluminum and/or lanthanum. 


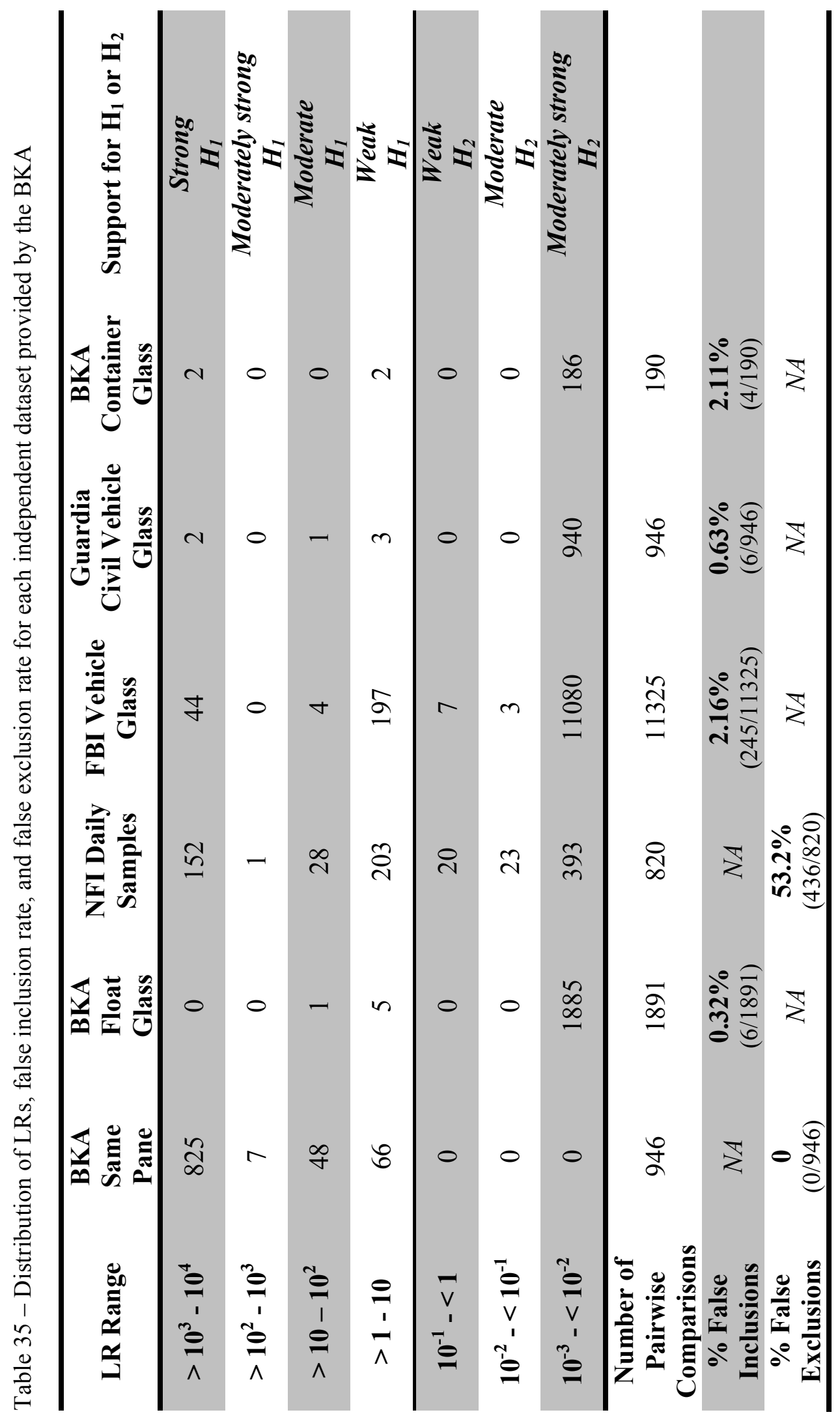




\subsubsection{BKA Float Glass}

For the BKA float glass collection, 6 out of 1,891 pairwise comparisons were falsely included, yielding a low false inclusion rate of $0.32 \%$. Five of the inclusions showed only weak support ( $\mathrm{LR}=4$ or 6 ) for the wrong hypothesis, an association, while the remaining pair showed moderate support $(\mathrm{LR}=36)$ for an association. In all cases, the pairs that were falsely included were manufactured by the same glass company (Pilkington or Saint Gobain).

\subsubsection{NFI Daily Samples}

The NFI dataset is a collection of glass samples manufactured by the same company over the span of 41 days; one sample was collected from each day so that, when comparing two samples, they were produced at least one day apart (i.e., no two samples were produced on the same day). It is known that the elemental composition of glass changes over time within a manufacturing plant. Therefore, it is expected that samples that were produced closer in time should be more strongly associated, while samples produced further apart in time should be more weakly associated or excluded. Figure 56 shows the distribution of the LR for samples produced 1 to $30+$ days apart. The different colors represent the weight of the evidence. For example, the green bars include pairs with a LR that shows a strong association $\left(10^{3}<\mathrm{LR} \leq 10^{4}\right)$. Note that the legend in the plot shows the $\log _{10}$ of the LR. It can be seen that for samples produced only 1 day apart, the majority of the pairs $(>90 \%)$ had an LR that showed strong support for an association. As the number of days produced apart increases for the two samples being compared, the strength of association begins to weaken; that is, the green bars shrink and the blue and 
purple bars enlarge. Additionally, as the number of days produced apart increases, the weak exclusions (yellow), moderate exclusions (orange), and moderately strong exclusions (red) increase as well. At about 11 days apart, half of the pairs are included and half are excluded. By 30 (or more) days apart, all samples are excluded.

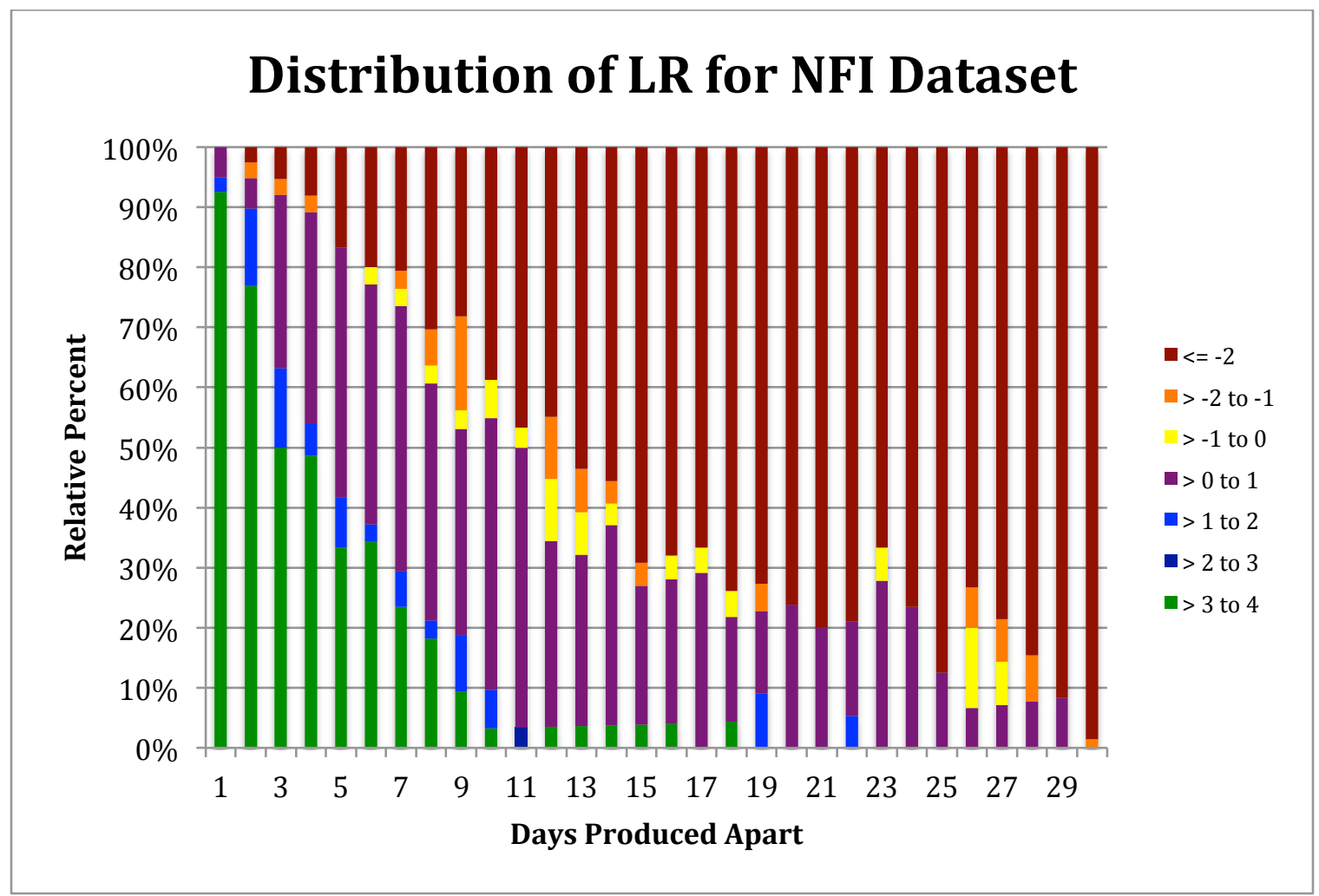

Figure 56 - Stacked column plot showing the distribution of LRs for the NFI Daily Samples set. The different colors represent the magnitude of the LR from a strong association (green) to a moderately strong exclusion (red). Note that the LRs are expressed in $\log _{10}$ in the legend.

Table 35 shows that the false exclusion rate for the NFI dataset is a high $53.2 \%$ (436 excluded pairs out of 820 total pairs). However, this value is misleading since it is difficult to establish when two samples are similar enough to be considered "samesource" or when they are different enough to be considered "different-source." Overall, 
the results were as expected: most pairs produced closely in time were associated and most pairs produced further apart in time were excluded.

\subsubsection{FBI Vehicle Glass}

The FBI vehicle set led to a relatively high false exclusion rate of $2.26 \%$ (245 out of 11,325). Thirty-five of those false inclusions, most of which showed strong support for an association, originated from the same vehicle but different vehicle window (rear window, windshield, or side windows). An additional 123 false inclusions were comparisons between different vehicles of the same make and/or year of manufacture. This left a remaining 87 pairs that originated from different vehicles, with different make, and different year of manufacture. Most of these 87 pairs showed only weak support $(\mathrm{LR}<$ 10) for an association; 6 pairs showed strong support for an association. Moreover, 67 of the 87 pairs were between samples from vehicles that were manufactured only one year apart; it is possible that, despite having different vehicle makes, the glass for these vehicles was obtained from the same manufacturing plant. Unfortunately, without the window sticker that discloses the glass manufacturer information, it is impossible to know whether the glass for these windows was indeed produced in the same glass plant and at around the same time. Overall, only 20 falsely included pairs originated from different vehicles of different make and produced at least 2 years apart.

\subsubsection{Guardia Civil Vehicle Glass}

For the Guardia Civil vehicle set, 6 out of 946 pairs were falsely included, resulting in a low false inclusion rate of $0.63 \%$. The two pairs that were strongly associated originated 
from the inner and outer windshield pane of the same vehicle, thus it is likely that the two glass panes were manufactured in the same glass plant. Two of the pairs that showed weak support also originated from the same vehicle, but from different windows (i.e., back window versus driver's front window). The two remaining false inclusions originated from different vehicles and had a different vehicle make and model: a Honda Civic versus a Mercedes 190 and a Volvo 740 versus a Mercedes 190. These last two pairs may have originated from the same glass manufacturer (the year of manufacture is unknown) or the glass samples may have coincidentally had a similar elemental profile.

\subsubsection{BKA Container Glass}

For the BKA container glass set, 4 pairs were falsely included $(2.11 \%$ false inclusion rate). Two of these pairs showed weak support ( $L R=5$ or 6 ) for an association and the remaining two pairs showed strong support $(\mathrm{LR}=6,517)$ for an association. All four false inclusions were comparisons between samples manufactured by the same company (Wiegand). One of the pairs that showed a weak association and one of the pairs that showed a strong association was a comparison between different types of product (champagne bottle versus beer bottle); however, the different types of bottles had the same color (green). It should be noted that the BKA database is comprised of float glass, thus it may not be an appropriate database for the calculation of a likelihood ratio for non-float (e.g., container) glass. 


\subsection{Conclusions for Analysis and Interpretation of LA-ICP-MS Glass Data}

This chapter evaluated the performance of a continuous likelihood ratio (LR) for the interpretation of forensic glass evidence. The Multivariate Kernel (MVK) model was shown to provide good discrimination, but the model ultimately led to an unreasonably large (or small) weight of evidence. Thus, the Pool Adjacent Algorithm (PAV) was implemented for the calibration of the LR and to impose a lower and upper limit to the LR output. A second calibration approach (the ELUB approach) was also assessed, however, the PAV algorithm performed better overall. Two databases were used for the evaluation of the PAV-calibrated LR: the FIU vehicle database and the BKA casework database. As expected, the BKA casework database resulted in better performance; this is because the BKA casework database includes a variety of samples obtained from actual casework samples collected over a long period of time, while the FIU vehicle database is a limited set of windshield glass from vehicles sampled in one location (the $M \& M$ Salvage Yard), many of which had the same make, model, and/or year of manufacture. Therefore, the BKA database has a higher between-source variation, which ultimately results in a stronger weight of evidence.

Excluding problematic samples from the databases led to improved results. For example, removing outer panes that were indistinguishable from the inner pane of the same vehicle in the FIU database led to stronger associations for same-source pairs and stronger exclusions for different-source pairs. Removing suspected non-float samples from the BKA casework database led to improved error rates. These results suggest that the proper selection of the background database is an important step for the calculation of a likelihood ratio. Unfortunately, a database that reflects the relevant population may not be 
available in many forensic laboratories. Further research is required in order to determine whether a single, shared database is appropriate for the calculation of a likelihood ratio. Since the PAV-calibrated BKA database performed best overall, this database was selected as the background population for several small independent datasets provided by the BKA. The calibrated LR performed well for most datasets. The relatively high false inclusion rate for two datasets (the FBI Vehicle set and the BKA Container set) could be explained by the similarity of the samples being compared; that is, the samples often had the same manufacturer or the same vehicle make, model, and/or year. The NFI set resulted in a high false exclusion rate. However, as explained earlier, this error rate is misleading since it is difficult to establish when two samples should be considered to be same-source or different-source pairs. The NFI set showed stronger associations for samples that were produced more closely in time and stronger exclusions for samples produce further apart in time. These results are an excellent demonstration of the weight of evidence provided by the likelihood ratio approach. On the other hand, match criteria result in a binary conclusion ("match" versus "non-match") and do not provide the strength of an association or exclusion.

Validation studies for the likelihood ratio approach are needed prior to implementing this approach in forensic casework. Ideally, each forensic laboratory would generate its own glass database from the relevant population (i.e., glass from the lab's surrounding area) and evaluate the performance of its database using known samples. Moreover, each lab may require a different element menu depending on the reproducibility of each element; this is discussed in more detail in the next chapter. Unfortunately, it is unlikely that every forensic lab would be able to generate a database that is sufficiently large for the 
calculation of a likelihood ratio. Thus, the second best option would be the use of a common database. The following chapter presents the results of an inter-laboratory study in which three PAV-calibrated databases (BKA, FIU, and BKA + FIU) were used to calculate the likelihood ratio for data collected by participating laboratories. 


\section{GLASS LA-ICP-MS INTER-LABORATORY STUDY}

An inter-laboratory study was included as part of the glass project (referred to as the Glass Interpretation Working Group, GIWG) in order to standardize the reporting language used by forensic practitioners and to evaluate the performance of the likelihood ratio across laboratories. A total of three exercises, also known as "round robins," were completed for the inter-laboratory study. For each round robin, known and questioned samples (taken from the FIU vehicle collection) were sent to each participating laboratory, which would then analyze each glass fragment using LA-ICP-MS. The data was then sent to the coordinators (FIU) of the inter-laboratory study. The LR for every known and questioned sample pair was calculated using three databases as the background population: the BKA casework database, the FIU vehicle database, and the two databases combined. The elements listed in ASTM E2927 were included for LR calculations with the exception of hafnium for reasons discussed in Chapter 4. Each database was calibrated using a 10 -fold cross validation. That is, the database was split into ten groups and the un-calibrated LR for all pairwise comparisons within one group were calculated, while the remaining nine groups were used as the background population for the MVK model. As described in Chapter 4, same-source pairs were generated by comparing the first 3 replicates of a sample to the last 3 replicates of the same sample, while different-source pairs were generated by comparing the first 3 replicates of a sample to the last 3 replicates of a different sample. This procedure was then implemented for each of the ten groups. Once the un-calibrated LR was calculated for all possible comparison pairs within each group, the LRs were pooled together and 
used to train the PAV algorithm. The un-calibrated LR for the round robin data submitted by the participating laboratories was then calculated using each database as the background population for the MVK model and subsequently calibrated using the PAV transformation for each database. All calculations were executed using RStudio (version 1.0.143). The error rates for each inter-laboratory test are presented below.

\subsection{Inter-laboratory Study: Exercise 1}

A total of 11 laboratories, including FIU, participated in the first round robin. However, only five laboratories performed LA-ICP-MS analysis; the remaining laboratories used solution ICP-MS, solution ICP-OES, LIBS, $\mu \mathrm{XRF}$, and/or refractive index to characterize the known and questioned samples. The results presented will only focus on LA-ICP-MS since, for this project, only LA-ICP-MS glass databases were available for the calculation of likelihood ratios.

For the first round robin, 2 known $(\mathrm{K})$ samples, each with an inner and outer pane, and 2 questioned $(\mathrm{Q})$ samples, each taken from the outer pane of an automobile windshield, were sent to every participating laboratory. Three glass fragments were submitted for every $\mathrm{K}$ and $\mathrm{Q}$. The labs were asked to analyze each fragment in triplicate (i.e., a minimum of 3 replicate measurements per fragment as specified in ASTM E2927). ${ }^{115}$ The 3 fragments for each $\mathrm{K}$ were grouped together so that each $\mathrm{K}$ had a minimum of 9 replicate measurements. Unlike the $\mathrm{K}$ samples, the $\mathrm{Q}$ fragments were not grouped together, but treated independently. Table 36 shows the identity of each $\mathrm{K}$ and $\mathrm{Q}$ submitted to participating laboratories: K1 was sampled from a 2009 Mitsubishi Galant, K2 from a 2008 Subaru Impreza, Q1 from a 2009 Mitsubishi Galant, and Q2 from a 2008 
Subaru Impreza. As can be seen in Table 36, K2 and Q2 have the same Vehicle Identification Number (VIN) since they were sampled from the same vehicle, thus Q2 should be indistinguishable from the outer pane of K2. K1 and Q1 were sampled from similar vehicles: same make, same model, same year, and close VIN.

Table 36 - Identity of Known and Questioned samples included in round robin 1

\begin{tabular}{lcccc}
\hline \multicolumn{1}{c}{ Sample } & Make & Model & Year & VIN \\
\hline K1 (inner \& outer) & Mitsubishi & Galant & 2009 & 4A3AB36F39E024088 \\
K2 (inner \& outer) & Subaru & Impreza & 2008 & JF1GE61658H503418 \\
Q1 (outer) & Mitsubishi & Galant & 2009 & 4A3AB36F39E029145 \\
Q2 (outer) & Subaru & Impreza & 2008 & JF1GE61658H503418 \\
\hline
\end{tabular}

Figure 57 shows the LR for all $\mathrm{K}$ and Q comparisons using the BKA database. Each lab was given an alphabetic label in order to protect the identity of the participating laboratories. One lab (Lab C) did not analyze K1 inner since that sample was excluded from all others on the basis of physical characteristics. One lab (Lab D) processed the data twice: once using FGS 2 as the calibrator and once using NIST 612 as the calibrator. For most cases in which the $\mathrm{K}$ and Q originated from different vehicles (K1 inner/outer \& Q1, K1 inner/outer \& Q2, K2 inner/outer \& Q1), the LR was less than one, providing support for a non-association. Moreover, the LR was equal to the lower limit established by the PAV algorithm $(L R=0.00270)$. However, some labs weakly associated K1 outer with Q1; this is likely because $\mathrm{K} 1$ outer and Q1 originated from the outer pane of similar vehicles (see Table 36) and thus, it is unknown whether these samples should be associated. Indeed, using the ASTM E2927 comparison criterion, most labs associated $\mathrm{K} 1$ outer and Q1. Only two labs found differences in one or two elements ( $\mathrm{Li}$ and/or $\mathrm{Sr}$ ). 
All comparisons between $\mathrm{K}$ and $\mathrm{Q}$ samples that originated from vehicles with a different make and/or year of manufacture had a LR less than 1, resulting in a correct discrimination. For the case in which the $\mathrm{K}$ and $\mathrm{Q}$ originated from the same pane of the same vehicle (K2 outer and Q2), most labs (Labs B, D, and E) showed a LR that provided strong support for an association. Lab A provided relatively weak support for an association. However, this lab does not typically use potassium $(\mathrm{K})$ or iron $(\mathrm{Fe})$ in casework because the lab obtained poor external calibration curves for these two elements. If $\mathrm{K}$ and $\mathrm{Fe}$ are removed from the element menu, the LR for Lab A changes from a moderate association $(10<\mathrm{LR} \leq 100)$ to a strong association $(1000<\mathrm{LR} \leq$ 10,000). ${ }^{41}$ Lab C stated that their instrumentation at the time of analysis was not performing optimally and was leading to high RSDs. A closer inspection of Lab C's data revealed a high RSD (> 15\%) for potassium. After removing potassium, Lab C showed a strong association for all $\mathrm{K} 2$ outer and Q2 pairs. These results suggest that each laboratory may need to validate their own element menu based on their reproducibility for each element.

Overall, using the BKA database led to a false exclusion rate of 5.6\% (2/36 K2 \& Q2 pairs); after removing the problematic elements for certain labs, there were no false exclusions. The false inclusion rate (using only comparisons between different vehicles) was $5.9 \%(6 / 102)$, though, as stated earlier, all false inclusions were comparisons between similar vehicles. If the $\mathrm{K}$ and $\mathrm{Q}$ pairs from similar vehicles are not considered as different sources, then the calibrated LR yields no false inclusions. 


\begin{tabular}{|l|cccccc|}
\hline \multicolumn{7}{|c|}{ USING BKA DATABASE (N = 370 SAMPLES) } \\
\hline Comparison Pair & Lab A & Lab B & Lab C & Lab D & Lab D 2 & Lab E \\
\hline K1 Inner \& Q1.1 & 0.00270 & 0.00270 & NA & 0.00270 & 0.00270 & 0.0648 \\
K1 Inner \& Q1.2 & 0.00270 & 0.00270 & NA & 0.00270 & 0.00270 & 0.248 \\
K1 Inner \& Q1.3 & 0.00270 & 0.00270 & NA & 0.00270 & 0.00270 & 0.0858 \\
\hline K1 Inner \& Q2.1 & 0.00270 & 0.00270 & NA & 0.00270 & 0.00270 & 0.00270 \\
K1 Inner \& Q2.2 & 0.00270 & 0.00270 & NA & 0.00270 & 0.00270 & 0.00270 \\
K1 Inner \& Q2.3 & 0.00270 & 0.00270 & NA & 0.00270 & 0.00270 & 0.00270 \\
\hline K1 Outer \& Q1.1 & 0.0357 & 6 & 0.00270 & 0.0641 & 0.00270 & 0.0648 \\
K1 Outer \& Q1.2 & 4 & 9 & 0.00270 & 2 & 0.164 & 0.248 \\
K1 Outer \& Q1.3 & 0.00270 & 6 & 0.00270 & 0.113 & 4 & 0.0858 \\
\hline K1 Outer \& Q2.1 & 0.00270 & 0.00270 & 0.00270 & 0.00270 & 0.00270 & 0.00270 \\
K1 Outer \& Q2.2 & 0.00270 & 0.00270 & 0.00270 & 0.00270 & 0.00270 & 0.00270 \\
K1 Outer \& Q2.3 & 0.00270 & 0.00270 & 0.00270 & 0.00270 & 0.00270 & 0.00270 \\
\hline K2 Inner \& Q1.1 & 0.00270 & 0.00270 & 0.00270 & 0.00270 & 0.00270 & 0.00270 \\
K2 Inner \& Q1.2 & 0.00270 & 0.00270 & 0.00270 & 0.00270 & 0.00270 & 0.00270 \\
K2 Inner \& Q1.3 & 0.00270 & 0.00270 & 0.00270 & 0.00270 & 0.00270 & 0.00270 \\
\hline K2 Inner \& Q2.1 & 9 & 6517 & 36 & 6517 & 6517 & 6517 \\
K2 Inner \& Q2.2 & 6 & 6517 & 6 & 6517 & 6517 & 6517 \\
K2 Inner \& Q2.3 & 9 & 6517 & 0.00270 & 6517 & 6517 & 6517 \\
\hline K2 Outer \& Q1.1 & 0.00270 & 0.00270 & 0.00270 & 0.00270 & 0.00270 & 0.00270 \\
K2 Outer \& Q1.2 & 0.00270 & 0.00270 & 0.00270 & 0.00270 & 0.00270 & 0.00270 \\
K2 Outer \& Q1.3 & 0.00270 & 0.00270 & 0.00270 & 0.00270 & 0.00270 & 0.00270 \\
\hline K2 Outer \& Q2.1 & 16 & 6517 & 24 & 6517 & 6517 & 6517 \\
K2 Outer \& Q2.2 & 36 & 6517 & 6517 & 6517 & 6517 & 6517 \\
K2 Outer \& Q2.3 & 36 & 6517 & 0.00270 & 6517 & 6517 & 6517 \\
\hline
\end{tabular}

Figure 57 - Calibrated LR for all round robin $1 \mathrm{~K}$ and Q comparisons using the BKA database as the background population

Similar results were obtained when using the FIU database as the background population

Figure 58. Labs B, D, and E strongly associated K2 outer and Q2, with one exception for Lab D (using NIST 612 as the calibrator), in which one pair provided moderately strong support for an association $(\mathrm{LR}=107)$. Lab A provided weak support for an association for all three $\mathrm{K} 2$ outer and Q2 pairs, while Lab C provided a weak association for two pairs and an exclusion for one pair. As with the BKA database, the false exclusion rate 
using the FIU database was 5.6\%. Compared to the BKA database, more labs weakly associated the same pane side of similar vehicles (K1 outer and Q1), leading to a higher false inclusion rate of $15.7 \%$ (16/102). No false inclusions were obtained if the glass from similar vehicles is not considered to be different sources.

\begin{tabular}{|l|cccccc|}
\hline \multicolumn{7}{c|}{ USING FIU DATABASE (N = 333 SAMPLES) } \\
\hline Comparison Pair & Lab A & Lab B & Lab C & Lab D & Lab D 2 & Lab E \\
\hline K1 Inner \& Q1.1 & 0.00596 & 0.00608 & NA & 0.00608 & 0.00608 & 4 \\
K1 Inner \& Q1.2 & 0.00596 & 0.00608 & NA & 0.00608 & 0.00608 & 4 \\
K1 Inner \& Q1.3 & 0.00596 & 0.00608 & NA & 0.00608 & 0.00596 & 4 \\
\hline K1 Inner \& Q2.1 & 0.00596 & 0.00596 & NA & 0.00596 & 0.00596 & 0.00596 \\
K1 Inner \& Q2.2 & 0.00596 & 0.00596 & NA & 0.00596 & 0.00596 & 0.00596 \\
K1 Inner \& Q2.3 & 0.00596 & 0.00596 & NA & 0.00596 & 0.00596 & 0.00596 \\
\hline K1 Outer \& Q1.1 & 4 & 4 & 0.00608 & 4 & 4 & 4 \\
K1 Outer \& Q1.2 & 4 & 4 & 4 & 4 & 4 & 4 \\
K1 Outer \& Q1.3 & 4 & 4 & 0.00608 & 4 & 4 & 4 \\
\hline K1 Outer \& Q2.1 & 0.00596 & 0.00596 & 0.00608 & 0.00596 & 0.00596 & 0.00596 \\
K1 Outer \& Q2.2 & 0.00596 & 0.00596 & 0.00608 & 0.00596 & 0.00596 & 0.00596 \\
K1 Outer \& Q2.3 & 0.00596 & 0.00596 & 0.00608 & 0.00596 & 0.00596 & 0.00596 \\
\hline K2 Inner \& Q1.1 & 0.00596 & 0.00596 & 0.00608 & 0.00596 & 0.00596 & 0.00596 \\
K2 Inner \& Q1.2 & 0.00596 & 0.00596 & 0.00608 & 0.00596 & 0.00596 & 0.00596 \\
K2 Inner \& Q1.3 & 0.00596 & 0.00596 & 0.00608 & 0.00596 & 0.00596 & 0.00596 \\
\hline K2 Inner \& Q2.1 & 4 & 107 & 4 & 4881 & 4881 & 4881 \\
K2 Inner \& Q2.2 & 4 & 107 & 4 & 4881 & 4881 & 4881 \\
K2 Inner \& Q2.3 & 4 & 640 & 0.00608 & 4881 & 4881 & 4881 \\
\hline K2 Outer \& Q1.1 & 0.00596 & 0.00596 & 0.00608 & 0.00596 & 0.00596 & 0.00596 \\
K2 Outer \& Q1.2 & 0.00596 & 0.00596 & 0.00608 & 0.00596 & 0.00596 & 0.00596 \\
K2 Outer \& Q1.3 & 0.00596 & 0.00596 & 0.00608 & 0.00596 & 0.00596 & 0.00596 \\
\hline K2 Outer \& Q2.1 & 4 & 4881 & 4 & 4881 & 107 & 4881 \\
K2 Outer \& Q2.2 & 4 & 4881 & 4 & 4881 & 4881 & 4881 \\
K2 Outer \& Q2.3 & 4 & 4881 & 0.00608 & 4881 & 4881 & 4881 \\
\hline
\end{tabular}

Figure 58 - Calibrated LR for all round robin $1 \mathrm{~K}$ and Q comparisons using the FIU database as the background population

Finally, using the combined BKA and FIU database led to similar results as well (Figure 59). The false exclusion rate was $5.6 \%(2 / 36)$ and the false inclusion rate was $12.7 \%$ 
$(13 / 102)$. There were no false inclusions if the glass from similar vehicles is considered to be the same source. Labs A and C showed relatively weak support for an association for the comparison between K2 outer and Q2, likely because of the problematic elements mentioned earlier ( $\mathrm{K}$ and $\mathrm{Fe})$.

\begin{tabular}{|l|cccccc|}
\hline \multicolumn{7}{|c|}{ USING BKA \& FIU DATABASE (N = 703 SAMPLES) } \\
\hline Comparison Pair & Lab A & Lab B & Lab C & Lab D & Lab D 2 & Lab E \\
\hline K1 Inner \& Q1.1 & 0.00143 & 0.00143 & NA & 0.00143 & 0.00143 & 2 \\
K1 Inner \& Q1.2 & 0.00143 & 0.00143 & NA & 0.00143 & 0.00143 & 2 \\
K1 Inner \& Q1.3 & 0.00143 & 0.00143 & NA & 0.00143 & 0.00143 & 2 \\
\hline K1 Inner \& Q2.1 & 0.00143 & 0.00143 & NA & 0.00143 & 0.00143 & 0.00143 \\
K1 Inner \& Q2.2 & 0.00143 & 0.00143 & NA & 0.00143 & 0.00143 & 0.00143 \\
K1 Inner \& Q2.3 & 0.00143 & 0.00143 & NA & 0.00143 & 0.00143 & 0.00143 \\
\hline K1 Outer \& Q1.1 & 0.00143 & 8 & 0.00143 & 2 & 0.00143 & 2 \\
K1 Outer \& Q1.2 & 2 & 9 & 2 & 2 & 2 & 2 \\
K1 Outer \& Q1.3 & 0.00143 & 8 & 0.00143 & 2 & 2 & 2 \\
\hline K1 Outer \& Q2.1 & 0.00143 & 0.00143 & 0.00143 & 0.00143 & 0.00143 & 0.00143 \\
K1 Outer \& Q2.2 & 0.00143 & 0.00143 & 0.00143 & 0.00143 & 0.00143 & 0.00143 \\
K1 Outer \& Q2.3 & 0.00143 & 0.00143 & 0.00143 & 0.00143 & 0.00143 & 0.00143 \\
\hline K2 Inner \& Q1.1 & 0.00143 & 0.00143 & 0.00143 & 0.00143 & 0.00143 & 0.00143 \\
K2 Inner \& Q1.2 & 0.00143 & 0.00143 & 0.00143 & 0.00143 & 0.00143 & 0.00143 \\
K2 Inner \& Q1.3 & 0.00143 & 0.00143 & 0.00143 & 0.00143 & 0.00143 & 0.00143 \\
\hline K2 Inner \& Q2.1 & 8 & 238 & 23 & 9879 & 1453 & 9879 \\
K2 Inner \& Q2.2 & 2 & 238 & 8 & 9879 & 9879 & 9879 \\
K2 Inner \& Q2.3 & 8 & 238 & 0.00143 & 9879 & 1453 & 9879 \\
\hline K2 Outer \& Q1.1 & 0.00143 & 0.00143 & 0.00143 & 0.00143 & 0.00143 & 0.00143 \\
K2 Outer \& Q1.2 & 0.00143 & 0.00143 & 0.00143 & 0.00143 & 0.00143 & 0.00143 \\
K2 Outer \& Q1.3 & 0.00143 & 0.00143 & 0.00143 & 0.00143 & 0.00143 & 0.00143 \\
\hline K2 Outer \& Q2.1 & 23 & 9879 & 23 & 9879 & 1453 & 9879 \\
K2 Outer \& Q2.2 & 23 & 9879 & 70 & 9879 & 9879 & 9879 \\
K2 Outer \& Q2.3 & 23 & 9879 & 0.00143 & 9879 & 1453 & 9879 \\
\hline
\end{tabular}

Figure 59 - Calibrated LR for all round robin $1 \mathrm{~K}$ and Q comparisons using the combined BKA and FIU database as the background population

Although a fairly high false inclusion rate was obtained using each database (if similar vehicles are considered different sources), it should be noted that the calculated LR 
typically provided only weak support $(\mathrm{LR}<10)$ for an association. Thus, the LR is only weakly misleading and would provide little weight if this were a case.

Table 37 - Strength of association or discrimination for round robin 1 K2 outer and Q2

\begin{tabular}{ccccl}
\hline LR Range & BKA & FIU & BKA + FIU & \multicolumn{1}{c}{ Support for $\mathbf{H}_{\mathbf{1}}$ or $\mathbf{H}_{\mathbf{2}}$} \\
\hline$>\mathbf{1 0}^{\mathbf{3}}-\mathbf{1 0}$ & 25 & 20 & 21 & Strong $\left(\mathrm{H}_{1}\right)$ \\
$>\mathbf{1 0 0}-\mathbf{1 0}^{\mathbf{3}}$ & 0 & 4 & 3 & Moderately strong $\left(\mathrm{H}_{1}\right)$ \\
$>\mathbf{1 0}-\mathbf{1 0 0}$ & 5 & 0 & 6 & Moderate $\left(\mathrm{H}_{1}\right)$ \\
$>\mathbf{1}-\mathbf{1 0}$ & 4 & 10 & 4 & Weak $\left(\mathrm{H}_{1}\right)$ \\
\hline $\mathbf{0 . 1}-<\mathbf{1}$ & 0 & 0 & 0 & Weak $\left(\mathrm{H}_{2}\right)$ \\
$\mathbf{0 . 0 1}-<\mathbf{0 . 1}$ & 0 & 0 & 0 & Moderate $\left(\mathrm{H}_{2}\right)$ \\
$\mathbf{0 . 0 0 1}-<\mathbf{0 . 0 1}$ & 2 & 2 & 2 & Moderately strong $\left(\mathrm{H}_{2}\right)$ \\
\hline Correct & $\mathbf{9 4 . 4 \%}$ & $\mathbf{9 4 . 4 \%}$ & $\mathbf{9 4 . 4 \%}$ & \\
Association & & & & \\
\hline
\end{tabular}

Table 37 shows the strength of association or discrimination for glass pairs from the same vehicle, K2 and Q2, using each of the three databases. For all three databases, the two pairs that were excluded by Lab C showed moderately strong support for the incorrect hypothesis, $\mathrm{H}_{2}$. Although the three databases resulted in the same correct association, $94.4 \%$, the strength of association differed between databases. The BKA database provided the best overall results since a greater number of pairwise comparisons showed the maximum support for an association $(1000<\mathrm{LR} \leq 10,000)$. The FIU database led to the worst results overall since this database had the greatest number of weakly associated pairs. Finally, the results for the combined database lay in between the BKA database and FIU database.

It is suspected that the BKA database performed best because it exhibits the greatest between-source variation, thus pairwise comparisons between the same (or similar) samples would result in stronger associations. On the other hand, even after excluding the 
outer panes that are indistinguishable from the inner panes of the same vehicle, the FIU database exhibits much less variation between sources because it includes many vehicles that have the same make, model, and/or year (Appendix B). Therefore, weaker associations are obtained when using the FIU database as the background population. Since the combined database includes the BKA and FIU database, the between-source variation lies somewhere in between each individual database.

All participants were asked to analyze NIST 1831 as a control in order to determine whether different labs were obtaining consistent results. The NIST 1831 data for one lab was compared to the NIST 1831 data for another lab. A LR was calculated for all possible comparisons between labs: a total of 15 pairwise comparisons $(6 \times 5 \div 2)$. Regardless of which database was used as the background population, only 3 out of 15 pairs were associated and the strength of the associations were mostly weak to moderate. The associated pairs included: Lab B with Lab D, Lab B with Lab E, and Lab D with Lab E. Interestingly, the 3 associated pairs were comparisons between labs that use the same calibrator (FGS 2) and calibration technique (single-point). The remaining 12 pairs were comparisons between labs that use a different calibrator (FGS 2, NIST 612, etc.) and/or different calibration techniques (single-point versus multi-point calibration). When using the ASTM Criterion, Lab B was associated with Lab D and Lab D was associated with Lab E; Lab B was distinguished from Lab $\mathrm{E}$ on the basis of iron only. Like the LR results, the remaining 12 pairwise comparisons between labs were discriminated; the most discriminating elements were found to be iron $(\mathrm{Fe})$, magnesium $(\mathrm{Mg})$, and titanium (Ti). Other, less problematic, elements included: potassium (K), lithium (Li), and lanthanum (La). 
Figure 60 shows a control chart that compares the NIST 1831 concentration across participating laboratories for selected elements. The green dashed line represents the reported concentration $\left(R_{C}\right)$ listed in ASTM E2927, the yellow dashed lines represent \pm 2 $\times R_{C} \times s_{R} \div 100$, and the red dashed lines represent $\pm 3 \times R_{C} \times s_{R} \div 100$, where $s_{R}$ is the between-lab reproducibility reported in ASTM E2927. ${ }^{115}$ For the most part, all labs fell within the control limits, though iron is a clear exception. Iron was the only element that had a high overall RSD ( $>20 \%$ ), whereas all other elements had an RSD $<10 \%$. Lab C and Lab D 2 reported much lower concentrations for Fe; these two labs used NIST 612 as the calibrator, which may be an indication that the reported concentration of Fe in NIST 612 is inaccurate.

Since labs that use the same calibrator and calibration technique seemed to obtain more similar data, it was decided that for the second round robin all labs would be asked to use the same calibrator and calibration technique (single-point calibration using FGS 2). Additionally, FGS 1 was selected as a control for round robin 2, since it is more similar to the windshield glass for the known and questioned samples. It should be noted that while the calibration method is important for the calculation of a frequency using a match criterion, it is suspected that the calibration method does not significantly affect the LR. The FGS 2 and NIST 612 calibrated data for Lab D resulted in similar LRs. Therefore, it may not be necessary for labs to calibrate with the same method in order to use the same glass database for LR calculations. 

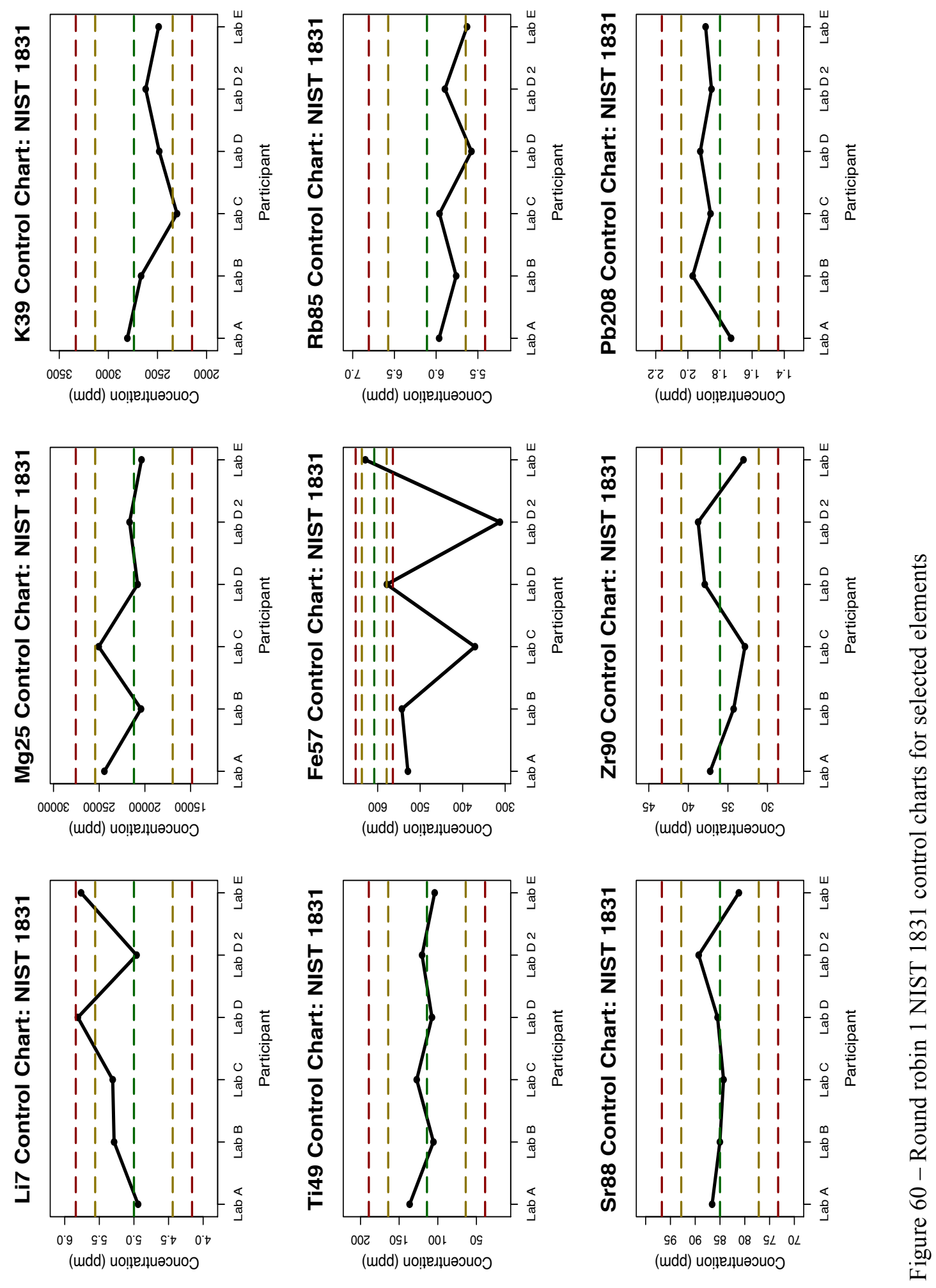


\subsection{Inter-laboratory Study: Exercise 2}

A total of 14 labs participated in the second round robin, 9 of which provided LA-ICPMS data. One K sample (inner and outer pane) and two Q samples were sent to each participant. Three glass fragments were submitted for each K and Q and participants were asked to analyze each fragment in triplicate. As can be seen in Table 38, the $\mathrm{K}$ was sampled from the inner and outer pane of a 2006 Honda Civic, Q1 from the same 2006 Honda Civic, and Q2 from the outer pane of a 2014 BMW 2 Series. Two of the Q1 fragments originated from the inner pane of the vehicle's windshield and thus should be indistinguishable from $\mathrm{K} 1$ inner, while the third fragment was sampled from the outer pane of the vehicle's windshield and thus should be indistinguishable from K1 outer. The K1 inner pane, K1 outer pane, and Q2 are all easily distinguished based on their elemental profile. Participants were asked to use FGS 2 as the calibrator for single-point calibration and FGS 1 and NIST 1831 as controls.

Table 38 - Identity of Known and Questioned samples included in round robin 2

\begin{tabular}{ccccc}
\hline Sample & Make & Model & Year & VIN \\
\hline K1 (inner \& outer) & Honda & Civic & 2006 & 2HGFG21506H707035 \\
Q1 (inner \& outer) & Honda & Civic & 2006 & 2HGFG21506H707035 \\
Q2 (outer) & BMW & 2 Series & 2014 & WBA1F5C58EVV98871 \\
\hline
\end{tabular}

Figure 61 shows the LR for all $\mathrm{K}$ and $\mathrm{Q}$ comparisons using each of the three databases. Lab B (which is the coordinating lab, FIU) only analyzed one Q2 fragment since it was decided after analysis that three fragments should be sent to participants. For all labs, the LR correctly supported an exclusion in the case where the $\mathrm{K}$ and $\mathrm{Q}$ originated from 
different vehicles (K1 and Q2). Moreover, as was the case for round robin 1, the LR was

typically equal to the lower limit imposed by the PAV algorithm.

\begin{tabular}{|l|ccccccccc}
\hline \multicolumn{10}{|c|}{ USING BKA DATABASE (N = 370 SAMPLES) } \\
\hline Comparison Pair & Lab A & Lab B & Lab C & Lab D & Lab E & Lab F & Lab G & Lab H & Lab I \\
\hline K1 Inner \& Q1.1 & 6517 & 6517 & 6517 & 6517 & 6517 & 6517 & 6517 & 6517 & 6517 \\
K1 Inner \& Q1.2 & 6517 & 6517 & 9 & 6517 & 6517 & 6517 & 6517 & 6517 & 6517 \\
K1 Inner \& Q1.3 & 0.00270 & 0.00270 & 0.00270 & 0.00270 & 0.00270 & 0.00270 & 0.00270 & 0.00270 & 0.00270 \\
\hline K1 Inner \& Q2.1 & 0.00270 & 0.00270 & 0.00270 & 0.00270 & 0.00270 & 0.00270 & 0.00270 & 0.00270 & 0.00270 \\
K1 Inner \& Q2.2 & 0.00270 & NA & 0.00270 & 0.00270 & 0.00270 & 0.00270 & 0.00270 & 0.00270 & 0.00270 \\
K1 Inner \& Q2.3 & 0.00270 & NA & 0.00270 & 0.00270 & 0.00270 & 0.00270 & 0.00270 & 0.00270 & 0.00270 \\
\hline K1 Outer \& Q1.1 & 0.00270 & 0.00270 & 0.00270 & 0.00270 & 0.00270 & 0.00270 & 0.00270 & 0.00270 & 0.00270 \\
K1 Outer \& Q1.2 & 0.00270 & 0.00270 & 0.00270 & 0.00270 & 0.00270 & 0.00270 & 0.00270 & 0.00270 & 0.00270 \\
K1 Outer \& Q1.3 & 6517 & 6517 & 6517 & 6517 & 6517 & 6517 & 6517 & 6517 \\
\hline K1 Outer \& Q2.1 & 0.00270 & 0.00270 & 0.00270 & 0.00270 & 0.00270 & 0.00270 & 0.00270 & 0.00270 & 0.00270 \\
K1 Outer \& Q2.2 & 0.00270 & NA & 0.00270 & 0.00270 & 0.00270 & 0.00270 & 0.00270 & 0.00270 & 0.00270 \\
K1 Outer \& Q2.3 & 0.00270 & NA & 0.00270 & 0.00270 & 0.00270 & 0.00270 & 0.00270 & 0.00270 & 0.00270 \\
\hline
\end{tabular}

\begin{tabular}{|c|c|c|c|c|c|c|c|c|c|}
\hline \multicolumn{10}{|c|}{ USING FIU DATABASE ( $N=333$ SAMPLES) } \\
\hline Comparison Pair & Lab A & Lab B & Lab C & Lab D & Lab E & Lab F & Lab G & Lab H & Lab I \\
\hline K1 Inner \& Q1.1 & 4881 & 4881 & 107 & 4881 & 12 & 4881 & 4881 & 4881 & 4881 \\
\hline K1 Inner \& Q1.2 & 4881 & 4881 & 12 & 4881 & 4881 & 107 & 4881 & 4881 & 107 \\
\hline K1 Inner \& Q1.3 & 0.00596 & 0.00596 & 0.00596 & 0.00608 & 0.00596 & 0.00608 & 0.00596 & 0.00596 & 0.00596 \\
\hline K1 Inner \& Q2.1 & 0.00596 & 0.00596 & 0.00596 & 0.00608 & 0.00596 & 0.00608 & 0.00596 & 0.00596 & 0.00596 \\
\hline K1 Inner \& Q2.2 & 0.00270 & NA & 0.00596 & 0.00608 & 0.00596 & 0.00608 & 0.00596 & 0.00596 & 0.00596 \\
\hline K1 Inner \& Q2.3 & 0.00270 & NA & 0.00596 & 0.00608 & 0.00596 & 0.00608 & 0.00596 & 0.00596 & 0.00596 \\
\hline K1 Outer \& Q1.1 & 0.00596 & 0.00596 & 0.00596 & 0.00608 & 0.00596 & 0.00608 & 0.00596 & 0.00596 & 0.00596 \\
\hline K1 Outer \& Q1.2 & 0.00596 & 0.00596 & 0.00596 & 0.00608 & 0.00596 & 0.00608 & 0.00596 & 0.00596 & 0.00596 \\
\hline K1 Outer \& Q1.3 & 4881 & 107 & 107 & 4881 & 12 & 107 & 107 & 4881 & 4881 \\
\hline K1 Outer \& Q2.1 & 0.00596 & 0.00596 & 0.00596 & 0.00608 & 0.00596 & 0.00608 & 0.00596 & 0.00596 & 0.00596 \\
\hline K1 Outer \& Q2.2 & 0.00270 & NA & 0.00596 & 0.00608 & 0.00596 & 0.00608 & 0.00596 & 0.00596 & 0.00596 \\
\hline K1 Outer \& Q2.3 & 0.00270 & NA & 0.00596 & 0.00608 & 0.00596 & 0.00608 & 0.00596 & 0.00596 & 0.00596 \\
\hline
\end{tabular}

\begin{tabular}{|c|c|c|c|c|c|c|c|c|c|}
\hline \multicolumn{10}{|c|}{ USING BKA \& FIU DATABASE (N = 703 SAMPLES) } \\
\hline Comparison Pair & Lab A & Lab B & Lab C & Lab D & Lab E & Lab F & Lab G & Lab H & Lab I \\
\hline K1 Inner \& Q1.1 & 9879 & 9879 & 1453 & 9879 & 28 & 9879 & 1453 & 9879 & 9879 \\
\hline K1 Inner \& Q1.2 & 9879 & 9879 & 23 & 9879 & 9879 & 238 & 1453 & 9879 & 76 \\
\hline K1 Inner \& Q1.3 & 0.00143 & 0.00143 & 0.00143 & 0.00143 & 0.00143 & 0.00143 & 0.00143 & 0.00143 & 0.00143 \\
\hline K1 Inner \& Q2.1 & 0.00143 & 0.00143 & 0.00143 & 0.00143 & 0.00143 & 0.00143 & 0.00143 & 0.00143 & 0.00143 \\
\hline K1 Inner \& Q2.2 & 0.00143 & NA & 0.00143 & 0.00143 & 0.00143 & 0.00143 & 0.00143 & 0.00143 & 0.00143 \\
\hline K1 Inner \& Q2.3 & 0.00143 & NA & 0.00143 & 0.00143 & 0.00143 & 0.00143 & 0.00143 & 0.00143 & 0.00143 \\
\hline K1 Outer \& Q1.1 & 0.00143 & 0.00143 & 0.00143 & 0.00143 & 0.00143 & 0.00143 & 0.00143 & 0.00143 & 0.00143 \\
\hline K1 Outer \& Q1.2 & 0.00143 & 0.00143 & 0.00143 & 0.00143 & 0.00143 & 0.00143 & 0.00143 & 0.00143 & 0.00143 \\
\hline K1 Outer \& Q1.3 & 9879 & 238 & 238 & 9879 & 78 & 238 & 238 & 9879 & 238 \\
\hline K1 Outer \& Q2.1 & 0.00143 & 0.00143 & 0.00143 & 0.00143 & 0.00143 & 0.00143 & 0.00143 & 0.00143 & 0.00143 \\
\hline K1 Outer \& Q2.2 & 0.00270 & NA & 0.00143 & 0.00143 & 0.00143 & 0.00143 & 0.00143 & 0.00143 & 0.00143 \\
\hline K1 Outer \& Q2.3 & 0.00270 & NA & 0.00143 & 0.00143 & 0.00143 & 0.00143 & 0.00143 & 0.00143 & 0.00143 \\
\hline
\end{tabular}

Figure 61 - LR results for all round robin $2 \mathrm{~K}$ and Q comparisons using the BKA (top), FIU (middle), and combined (bottom) database as the background population

All labs correctly associated two Q1 fragments with the inner pane of K1 and one Q1 fragment with the outer pane of $\mathrm{K} 1$, leading to a correct association of $100 \%$ regardless of which database was used as the background population. As was the case for round 
robin 1, the BKA database led to the best results: all labs strongly associated the three $\mathrm{K} 1$ inner/outer and Q1 fragments. The only exception was one pair for Lab C, which showed weak support $(\mathrm{LR}=9)$ for an association. Upon closer inspection, it was determined that this lab had a reproducibility issue for cerium; after removing Ce, this $\mathrm{K} 1$ inner and Q1 pair was strongly associated.

When using the FIU or the combined database as the background population, several labs (Labs B, C, E, F, G, and I) showed a relatively weak $(\mathrm{LR}<250)$ association for some pairwise comparisons. However, all of these labs were able to correctly associate K1 inner/outer and Q1 using the ASTM match criterion. Thus, it is suspected that the relatively weak associations are due to the lack of variation between sources for the FIU database and, to a lesser extent, the combined database. Lab E was the only lab that falsely excluded one pair (K1 inner and Q1.1) using the ASTM criterion; seven elements were found to be distinguishable, many of which were excluded because of the "fall off the cliff" effect. Lab E provided an explanation for the false exclusion: the replicate measurements were taken on a shoulder on the side of the glass fragment, rather than at the top, which may have affected the accuracy. When Lab E repeated their analyses, the pair was correctly associated using the ASTM criterion. Labs A and C have reported reproducibility issues with specific elements (potassium and iron). Finally, Lab I reported an extremely high RSD ( $>45 \%$ ) for lithium and a high RSD ( $>15 \%$ ) for lead for fragment Q1.2.

Table 39 shows a summary of the strength of association for K1 inner/outer and Q1 using each of the three different databases. As expected, the BKA database provided the best results: nearly all pairs were strongly associated and the one pair that was weakly 
associated was corrected after cerium was excluded. The FIU database and the combined database performed similarly. However, when comparing the magnitudes of the LR (Figure 61), it can be seen that the combined database generally resulted in larger LRs for pairs that should have been associated.

Table 39 - Strength of association or discrimination for round robin 2 K1 and Q1

\begin{tabular}{c|cccl}
\hline LR Range & BKA & FIU & BKA + FIU & \multicolumn{1}{|c}{ Support for $\mathbf{H}_{\mathbf{1}}$ or $\mathbf{H}_{\mathbf{2}}$} \\
\hline$>\mathbf{1 0} \mathbf{3}-\mathbf{1 0}$ & 26 & 17 & 17 & Strong $\left(\mathrm{H}_{1}\right)$ \\
$>\mathbf{1 0 0}-\mathbf{1 0}$ & 0 & 7 & 6 & Moderately strong $\left(\mathrm{H}_{1}\right)$ \\
$>\mathbf{1 0}-\mathbf{1 0 0}$ & 0 & 3 & 4 & Moderate $\left(\mathrm{H}_{1}\right)$ \\
$>\mathbf{1}-\mathbf{1 0}$ & 1 & 0 & 0 & Weak $\left(\mathrm{H}_{1}\right)$ \\
\hline $\begin{array}{c}\text { Correct } \\
\text { Association }\end{array}$ & $\mathbf{1 0 0} \%$ & $\mathbf{1 0 0 \%}$ & $\mathbf{1 0 0 \%}$ & \\
\hline
\end{tabular}

The control for round robin 2, FGS 1, was compared between laboratories in order to evaluate how similar the inter-lab concentrations were. The LR was calculated for all between-lab comparison pairs using each of the three databases as the background population. A total of 19 out of 36 pairwise comparisons were associated, yielding a low correct association of $52.8 \%$. The ASTM criterion revealed that cerium $(\mathrm{Ce})$, zirconium $(\mathrm{Zr})$, and rubidium $(\mathrm{Rb})$ were the most common distinguishing elements. However, several other elements were found to be discriminating across laboratories: iron $(\mathrm{Fe})$, lithium (Li), lanthanum (La), aluminum (Al), strontium $(\mathrm{Sr})$, neodymium $(\mathrm{Nd})$, and lead $(\mathrm{Pb})$. Rubidium was only a problematic element for Lab F, which was the only laboratory that obtained a concentration close to the reported value of $8.6 \mathrm{ppm}$ (parts per million); all other laboratories consistently obtained a value closer to $7 \mathrm{ppm}$ (Figure 62). Aluminum was only problematic for Lab B, which had a known issue with the instrumental pulse/analog calibration. After removing $\mathrm{Ce}, \mathrm{La}$, and $\mathrm{Rb}$, the correct 
association increased to $83.3 \%$. Although, $\mathrm{Zr}$ was a problematic element using the ASTM criterion, removing $\mathrm{Zr}$ had no effect on the LR results; this may be because there is high variation for within- and between-samples in the background database for $\mathrm{Zr}$.

Figure 62 shows the FGS 1 control charts for selected elements. The green dashed line represents the reported value and the yellow and red dashed lines represent \pm 2 and \pm 3 reported standard deviations, respectively. ${ }^{73}$ Compared to other elements, potassium (K) and zirconium $(\mathrm{Zr})$ varied widely across laboratories. With a few exceptions, the remaining elements are fairly consistent between labs. The majority of the elements for FGS 1 resulted in an overall RSD $<10 \%$; the only exception was rubidium, with an RSD of $10.6 \%$. Overall, using FGS 2 as the calibrator and FGS 1 as the control led to better performance than the other calibration methods and controls used in round robin 1 . Therefore, it was decided that a single-point calibration using FGS 2 and the control sample FGS 1 would also be utilized for round robin 3 . 

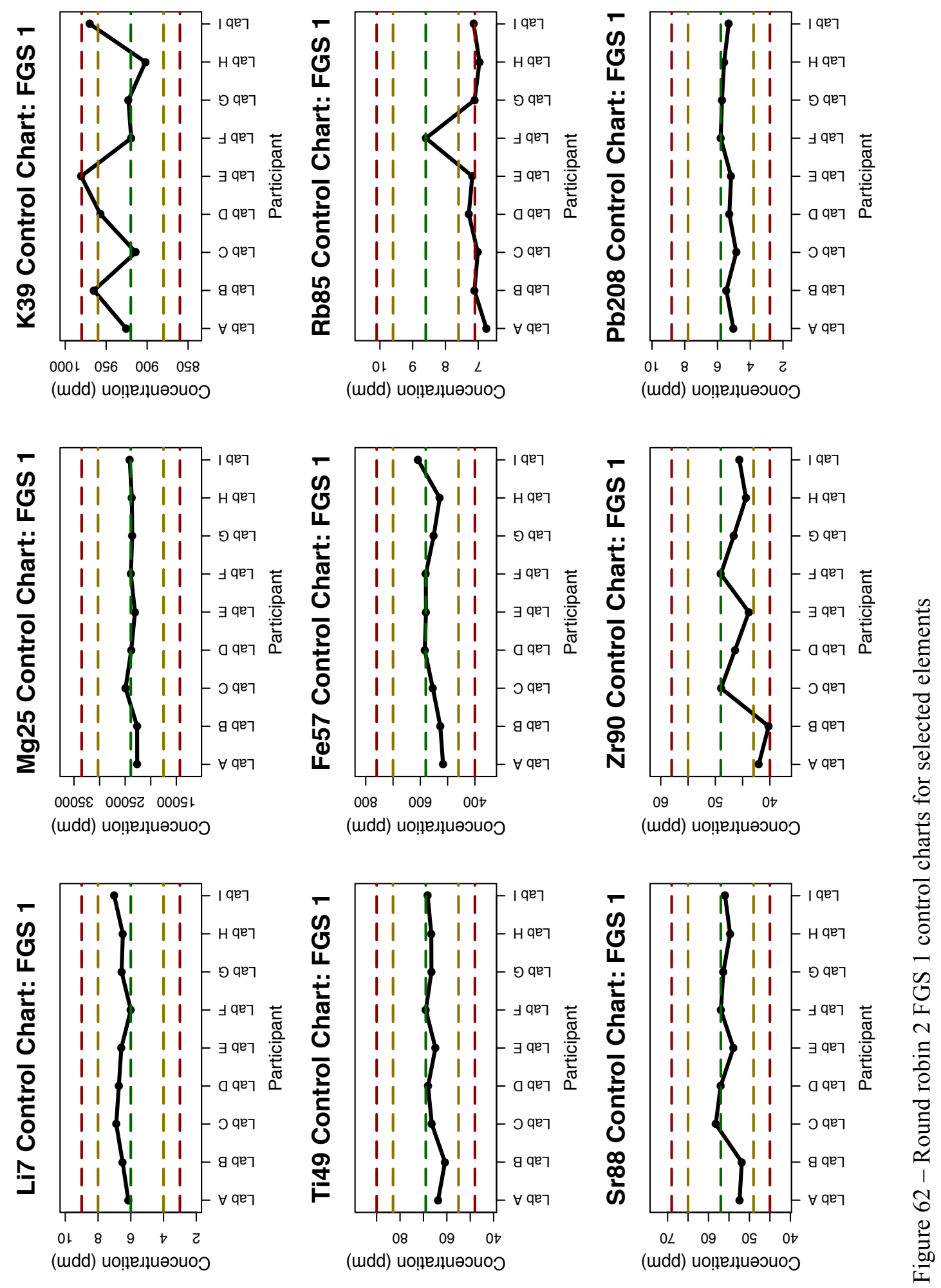


\subsection{Inter-laboratory Study: Exercise 3}

A total of 16 labs participated in the second round robin, 10 of which provided LA-ICPMS data. One K sample (inner and outer pane) and two Q samples were sent to each participant. Three glass fragments were submitted for each K and Q and participants were asked to analyze each fragment in triplicate. As can be seen in Table 40, the $\mathrm{K}$ was sampled from the inner and outer pane of a 2007 Honda Civic, Q1 from the outer pane of the same 2007 Honda Civic, and Q2 from the outer pane of a 2009 Mercedes R Series. All three Q1 fragments originated from the outer pane of the vehicle's windshield and thus should be indistinguishable from $\mathrm{K} 1$ outer. The $\mathrm{K} 1$ inner pane, $\mathrm{K} 1$ outer pane, and Q2 are all easily distinguished based on their elemental profile. Participants were asked to use FGS 2 as the calibrator for single-point calibration and FGS 1 and NIST 1831 as controls.

Table 40 - Identity of Known and Questioned samples included in round robin 3

\begin{tabular}{ccccc}
\hline Sample & Make & Model & Year & VIN \\
\hline K1 (inner \& outer) & Honda & Civic & 2007 & 2HGFG12607H511521 \\
Q1 (outer) & Honda & Civic & 2007 & 2HGFG12607H511521 \\
Q2 (outer) & Mercedes & R Series & 2009 & 4JGCB65E59A094913 \\
\hline
\end{tabular}

Figure 63 shows the LR for all $\mathrm{K}$ and $\mathrm{Q}$ comparisons using each of the three databases. All labs correctly excluded samples that originated from different vehicles (K1 and Q2) as well as Q1 from the inner pane of K1, regardless of which database was used as the background database. Most of the correct exclusions had a LR equal to the lower limit established by the PAV algorithm. 


\begin{tabular}{|c|c|c|c|c|c|c|c|c|c|c|}
\hline Comparison Pair & Lab A & Lab B & Lab C & Lab D & Lab E & Lab F & Lab G & Lab H & Lab I & Lab J \\
\hline K1 Inner \& Q1.1 & 0.00270 & 0.00270 & 0.00270 & 0.00270 & 0.00270 & 0.00270 & 0.00270 & 0.00270 & 0.00270 & 0.00270 \\
\hline K1 Inner \& Q1.2 & 0.00270 & 0.00270 & 0.00270 & 0.00270 & 0.00270 & 0.00270 & 0.00270 & 0.00270 & 0.00270 & 0.00270 \\
\hline K1 Inner \& Q1.3 & 0.00270 & 0.00270 & 0.00270 & 0.00270 & 0.00270 & 0.00270 & 0.00270 & 0.00270 & 0.00270 & 0.00270 \\
\hline K1 Inner \& Q2.1 & 0.00270 & 0.00270 & 0.00270 & 0.00270 & 0.00270 & 0.00270 & 0.00270 & 0.00270 & 0.00270 & 0.00270 \\
\hline K1 Inner \& Q2.2 & 0.00270 & 0.00270 & 0.00270 & 0.00270 & 0.00270 & 0.00270 & 0.00270 & 0.00270 & 0.00270 & 0.00270 \\
\hline K1 Inner \& Q2.3 & 0.00270 & 0.00270 & 0.00270 & 0.00270 & 0.00270 & 0.00270 & 0.00270 & 0.00270 & 0.00270 & 0.00270 \\
\hline K1 Outer \& Q1.1 & 6517 & 6517 & 36 & 6517 & 6517 & 6517 & 6517 & 6517 & 9 & 6517 \\
\hline K1 Outer \& Q1.2 & 6 & 6517 & 6517 & 6517 & 6517 & 6517 & 6517 & 6517 & 6517 & 6517 \\
\hline K1 Outer \& Q1.3 & 6 & 6517 & 6517 & 6517 & 6517 & 6517 & 6517 & 6517 & 6517 & 6517 \\
\hline K1 Outer \& Q2.1 & 0.00270 & 0.00270 & 0.00270 & 0.00270 & 0.00270 & 0.00270 & 0.00270 & 0.00270 & 0.00270 & 0.00270 \\
\hline K1 Outer \& Q2.2 & 0.00270 & 0.00270 & 0.00270 & 0.00270 & 0.00270 & 0.00270 & 0.00270 & 0.00270 & 0.00270 & 0.00270 \\
\hline K1 Outer \& Q2.3 & 0.00270 & 0.00270 & 0.00270 & 0.00270 & 0.00270 & 0.00270 & 0.00270 & 0.00270 & 0.00270 & 0.00270 \\
\hline
\end{tabular}

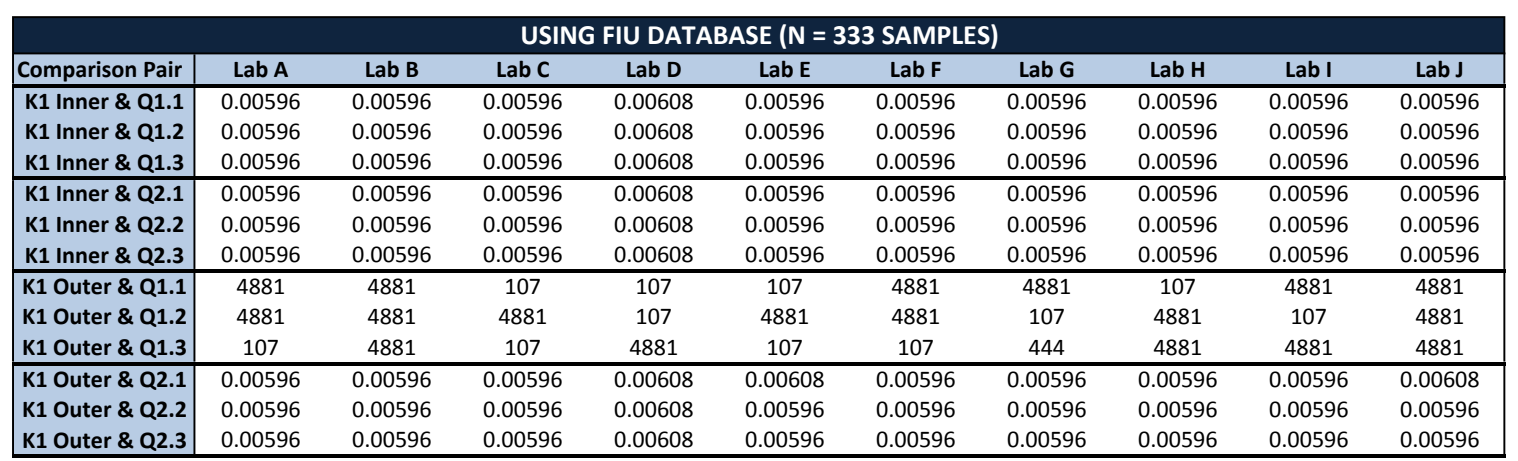

\begin{tabular}{|c|c|c|c|c|c|c|c|c|c|c|}
\hline \multicolumn{11}{|c|}{ USING BKA \& FIU DATABASE ( $N=703$ SAMPLES) } \\
\hline Comparison Pair & Lab A & Lab B & Lab C & Lab D & Lab E & Lab F & Lab G & Lab H & Lab I & Lab J \\
\hline K1 Inner \& Q1.1 & 0.00143 & 0.00143 & 0.00143 & 0.00143 & 0.00143 & 0.00143 & 0.00143 & 0.00143 & 0.00143 & 0.00143 \\
\hline K1 Inner \& Q1.2 & 0.00143 & 0.00143 & 0.00143 & 0.00143 & 0.00143 & 0.00143 & 0.00143 & 0.00143 & 0.00143 & 0.00143 \\
\hline K1 Inner \& Q1.3 & 0.00143 & 0.00143 & 0.00143 & 0.00143 & 0.00143 & 0.00143 & 0.00143 & 0.00143 & 0.00143 & 0.00143 \\
\hline K1 Inner \& Q2.1 & 0.00143 & 0.00143 & 0.00143 & 0.00143 & 0.00143 & 0.00143 & 0.00143 & 0.00143 & 0.00143 & 0.00143 \\
\hline K1 Inner \& Q2.2 & 0.00143 & 0.00143 & 0.00143 & 0.00143 & 0.00143 & 0.00143 & 0.00143 & 0.00143 & 0.00143 & 0.00143 \\
\hline K1 Inner \& Q2.3 & 0.00143 & 0.00143 & 0.00143 & 0.00143 & 0.00143 & 0.00143 & 0.00143 & 0.00143 & 0.00143 & 0.00143 \\
\hline K1 Outer \& Q1.1 & 9879 & 9879 & 76 & 238 & 238 & 1453 & 1453 & 238 & 238 & 1453 \\
\hline K1 Outer \& Q1.2 & 238 & 238 & 238 & 238 & 9879 & 238 & 238 & 9879 & 238 & 9879 \\
\hline K1 Outer \& Q1.3 & 238 & 9879 & 238 & 9879 & 238 & 238 & 238 & 9879 & 1453 & 9879 \\
\hline K1 Outer \& Q2.1 & 0.00143 & 0.00143 & 0.00143 & 0.00143 & 0.00143 & 0.00143 & 0.00143 & 0.00143 & 0.00143 & 0.00143 \\
\hline K1 Outer \& Q2.2 & 0.00143 & 0.00143 & 0.00143 & 0.00143 & 0.00143 & 0.00143 & 0.00143 & 0.00143 & 0.00143 & 0.00143 \\
\hline K1 Outer \& Q2.3 & 0.00143 & 0.00143 & 0.00143 & 0.00143 & 0.00143 & 0.00143 & 0.00143 & 0.00143 & 0.00143 & 0.00143 \\
\hline
\end{tabular}

Figure 63 - LR results for all round robin $3 \mathrm{~K}$ and Q comparisons using the BKA (top), FIU (middle), and combined (bottom) database as the background population

All labs correctly associated Q1 with the outer pane of $\mathrm{K} 1$, leading to $100 \%$ correct association. However, the strength of the association depended on which database was used as the background population. When the BKA database was used as the background population, all but four pairs showed strong support for an association, with the LR equal to the upper limit established by the PAV algorithm $(\mathrm{LR}=6517)$. A closer inspection of the four weakly $(\mathrm{LR}=6$ or 9$)$ or moderately $(\mathrm{LR}=36)$ associated pairs revealed several 
problematic elements. Lab A and Lab I showed high RSDs with lithium; removing lithium resulted in a strong association for all problematic pairs. Lab C showed high RSDs for rubidium and lanthanum; removing both elements resulted in a strong association for the problematic pair.

Table 41 - Strength of association or discrimination for round robin $3 \mathrm{~K} 1$ and Q1

\begin{tabular}{ccccl}
\hline LR Range & BKA & FIU & BKA + FIU & \multicolumn{1}{c}{ Support for $\mathbf{H}_{\mathbf{1}}$ or $\mathbf{H}_{\mathbf{2}}$} \\
\hline$>\mathbf{1 0}^{\mathbf{3}}-\mathbf{1 0}^{\mathbf{4}}$ & 26 & 18 & 13 & Strong $\left(\mathrm{H}_{1}\right)$ \\
$>\mathbf{1 0 0}-\mathbf{1 0}^{\mathbf{3}}$ & 0 & 12 & 16 & Moderately strong $\left(\mathrm{H}_{1}\right)$ \\
$>\mathbf{1 0}-\mathbf{1 0 0}$ & 1 & 0 & 1 & Moderate $\left(\mathrm{H}_{1}\right)$ \\
$>\mathbf{1}-\mathbf{1 0}$ & 3 & 0 & 0 & Weak $\left(\mathrm{H}_{1}\right)$ \\
\hline $\begin{array}{c}\text { Correct } \\
\text { Association }\end{array}$ & $\mathbf{1 0 0 \%}$ & $\mathbf{1 0 0 \%}$ & $\mathbf{1 0 0} \%$ & \\
\hline
\end{tabular}

Table 41 shows the strength of association for all K1 outer and Q1 comparisons using each of the three databases. When using the FIU database, all pairs showed either a moderately strong or strong association. Although no pairs showed a weak or moderate association, only $60 \%$ (18 out of 30 ) of the pairs had a LR equal to the upper limit established by the PAV algorithm. With the combined (BKA + FIU) database, even fewer pairs (13 out of 30) showed a strong association; no pairs had a LR equal to the upper limit established by the PAV algorithm (LR = 16,471). As discussed earlier, the greater number of strong associations observed for the BKA database is likely the result of the greater between-source variation.

Figure 64 shows the FGS 1 control charts for selected elements. The green dashed line represents the reported value and the yellow and red dashed lines represent \pm 2 and \pm 3 reported standard deviations, respectively. ${ }^{73}$ As with round robin 2, potassium and, to a 
lesser extent, zirconium showed higher variation between labs compared to the other elements. All labs consistently obtained a concentration lower than the reported value for rubidium.

When comparing FGS 1 across laboratories using the ASTM criterion, 53 out of 90 pairs were associated $(58.9 \%$ correct association). Most falsely excluded pairs were distinguished by iron, cerium, and/or lead. Other problematic elements included zirconium, lanthanum, and titanium. Using the calibrated LR with the BKA database as the background population led to a correct association of $68.9 \%$ (31 out of 45 pairs). However, of these 31 pairs only seven showed a strong association $(L R=6517)$; the remaining pairs had a LR that was less than 40. Removing iron, cerium, and lead improved the correct association only slightly, from $68.9 \%$ to $73.3 \%$. Further excluding potassium (known to be an issue for Lab A) resulted in a correct association of $82.2 \%$; nonetheless, Lab A was still discriminated from most of the remaining labs. Thus, even after using the same calibration method (single-point with FGS 2), using a matrixmatched control (FGS 1), and excluding problematic elements, the element concentrations across laboratories are different enough to yield false exclusions. These results indicate that a single shared database may not be appropriate for the calculation of the LR; instead it may be more suitable for each lab to have its own glass database. With the latter approach, the inter-laboratory variation would not need to be taken into account, while the intra-laboratory variation for elements with poor reproducibility would be taken into account. 

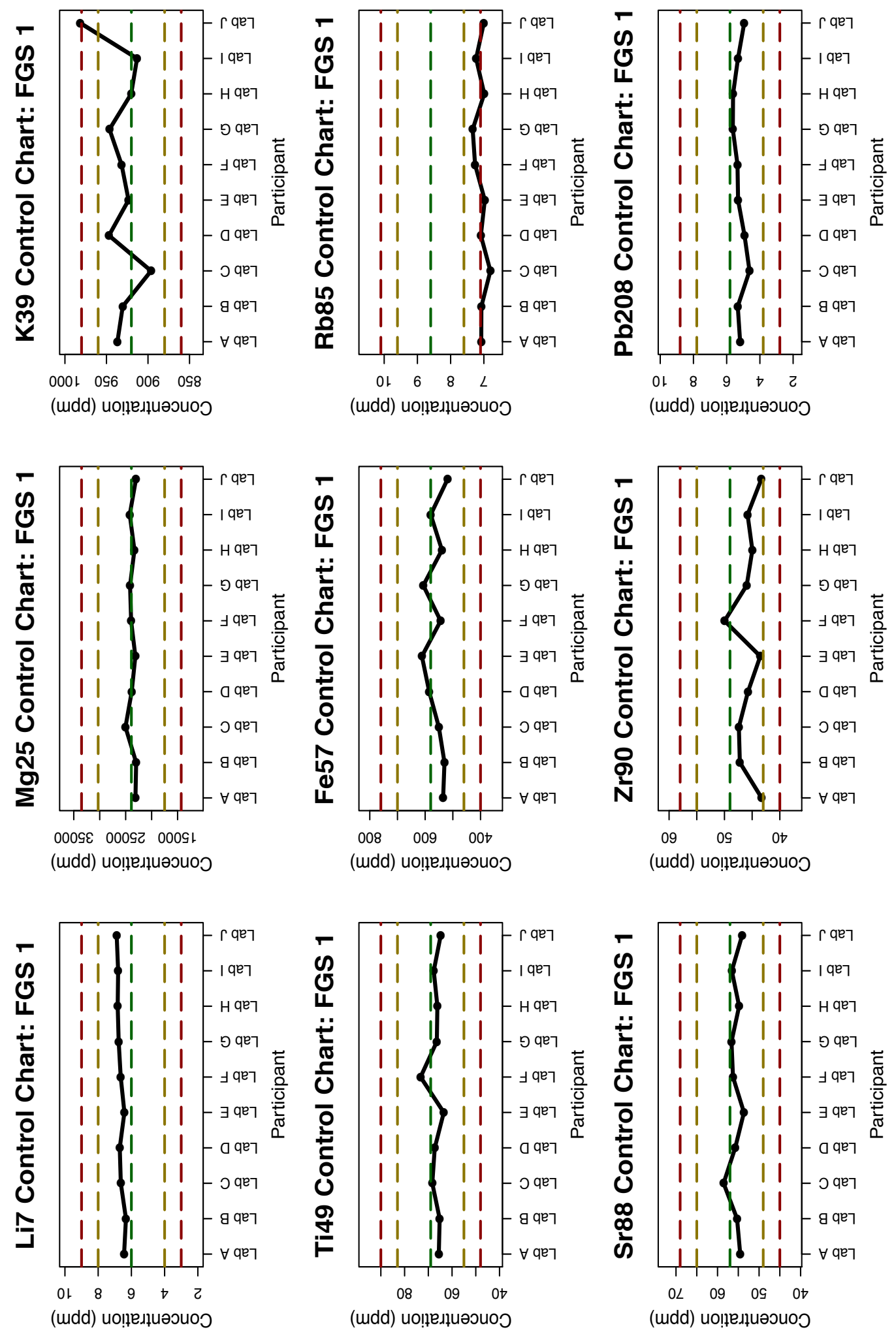

$\frac{a}{2}$

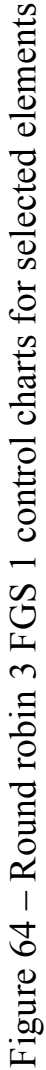




\subsection{Conclusions for Glass LA-ICP-MS Inter-Laboratory Study}

This chapter discussed the results of a calibrated likelihood ratio for the interpretation of forensic glass evidence. An inter-laboratory study was implemented in order to assess the performance of the LR using several different background databases. The Multivariate Kernel model, proposed by Aitken and Lucy, followed by a calibration using the Pool Adjacent Violators algorithm was employed for the calculation of the LR.

For round robin 1, two pairs were falsely excluded, leading to a correct association of $94.4 \%$ regardless of which database was used as the background population. Round robin 2 and 3 resulted in no false exclusions (100\% correct association). For all round robins, pairs that originated from different vehicles were correctly excluded; the exceptions to this were several weak inclusions between similar vehicles (same make, model, or year) in round robin 1. Unfortunately, it is unknown whether the glass windshields for these two vehicles were manufactured in the same glass plant at around the same time.

Overall, using the BKA casework database as the background population resulted in a greater number of strong associations for same-source comparisons. This is likely the result of greater variation between sources when compared to the FIU or the combined (BKA + FIU) database. As discussed earlier in the chapter, the FIU database contains many samples from similar vehicles collected within a short time frame, while the BKA database includes casework samples collected over many years. Although the same error rates were observed for all three databases, it seems clear that the selection of the background population significantly affects the weight of evidence. As such, an important step for the calculation of a LR is the proper selection of a database that accurately reflects the relevant population. 
In all three round robins, a few labs showed reproducibility issues for certain elements. These reproducibility issues led to weak associations for samples that should have been strongly associated. Lab A has known problems with potassium and iron and therefore does not use either element for discrimination in casework. Other labs (Lab A and I) showed issues with potassium, cerium, lithium, lanthanum, and/or rubidium. These results suggest that each forensic lab may need its own limited element menu based on the lab's reproducibility and accuracy for each element. Alternatively, each lab may generate its own glass database thereby accounting for the poor reproducibility of problematic elements.

In round robin 1, labs that calibrated their data using a single-point calibration with FGS 2 obtained more similar results than those that calibrated using alternative standards or with a different calibration method. Thus, for the following two round robins, singlepoint calibration with FGS 2 was specified in the instructions. Additionally, the standard FGS 1 (rather than NIST 1831) was selected as the control since it is a better matrix match to the Known and Questioned samples distributed to participating labs. Overall, the results for FGS 1 were more reproducible across laboratories compared to the NIST 1831 results. However, it is clear that there are significant differences in the concentration of FGS 1 obtained between labs; only about half of the comparison pairs were associated and the majority of these inclusions showed weak support for an association. These inter-lab differences in concentration are unsurprising since glass databases take into account the intra-day variation for same-source samples and inter-day variation for different-source samples; that is, variations across laboratories are unaccounted for. Still, this may not be an issue for the likelihood ratio since casework 
samples are analyzed in the same laboratory and usually on the same day. Indeed, the majority of same-source comparisons showed strong or moderately strong support for an association for all three round robins.

Only one of the participating laboratories currently uses a likelihood ratio for LA-ICPMS data in casework. For two samples to be considered indistinguishable 16 out of 18 elements must "match" using their match criterion and the LR must show an association $\left(\log _{10} L R>4\right)$. The LR is presently treated as a score since it is un-calibrated, though efforts to calibrate their casework database have recently been published. ${ }^{44-45}$ Since the LR is a relatively new approach in the U.S., using a match criterion combined with the LR has its merits. Much research has been published on the use of a match criterion for the comparison of casework samples, thus forensic analysts are familiar with this frequentist approach. On the other hand, analysts are unfamiliar with the much more complex LR approach. Adding the LR approach would allow analysts to compare this new approach to the previously established approach. A gentle introduction to the LR may help U.S. practitioners overcome their reservations for the LR approach. 


\section{OVERALL CONCLUSIONS}

The first part of this study focused on the evaluation of SEM-EDS for the analysis of four types of printing inks: inkjet, toner, offset, and intaglio. A total of 319 ink specimens were collected and analyzed using SEM-EDS. Ink sample pairs of the same ink type were then compared using spectral overlay after spectral normalization. Despite its relatively high limit of detection ( 1000 ppm), SEM-EDS offered high discrimination capabilities for toner inks (97.1\%) and intaglio inks (98.2\%). However, the technique showed limited utility for inkjet and offset inks. Compared to toner and intaglio inks, few elements were detectable in the inkjets, resulting in poor discrimination (78.4\%). Although many elements were detected in the offset inks, relatively low discrimination $(80.3 \%)$ was obtained because of the thin ink film, which led to high paper contribution to the ink signal.

The SEM-EDS results were compared to the more sensitive elemental technique LA-ICPMS. The lower detection limit of LA-ICP-MS resulted in a greater number of detectable elements and improved discrimination capabilities ( $>99 \%$ for all ink types). However, for some toner, inkjet, and offset samples, SEM-EDS provided complementary information and was able to discriminate some pairs that were indistinguishable using LA-ICP-MS. Elements that are difficult to detect using LA-ICP-MS because of polyatomic interferences or high ionization potential (e.g. $\mathrm{Si}, \mathrm{Cl}, \mathrm{S}, \mathrm{Fe}$ ) were easily detected using SEM-EDS. Moreover, SEM-EDS provides imaging capabilities, which was found to be useful for the classification of toners. Some toner inks that were indistinguishable by their elemental profile were easily distinguished based on their 
particle shape and size. There were morphological differences seen in offset and intaglio inks as well. However, SEM imaging of these two ink types did not provide any additional discrimination.

The majority of the indistinguishable toner and inkjet pairs were comparisons between inks of the same brand and/or color. However, many indistinguishable pairs were comparisons between black and magenta or yellow and magenta inks, suggesting a similarity in their inorganic components. Cyan inks were easily distinguished from the other colors because of the presence of copper (which likely originates from the most commonly used blue colorant, copper phthalocyanine).

Since the toner and inkjet inks were collected from pure color cartridges (cyan, magenta, yellow, and black), the discrimination between ink samples of the same color was also calculated. This provides a more reasonable evaluation of each technique's discrimination capabilities since inks of different colors are easily distinguished by visual inspection alone. SEM-EDS provided a discrimination between $83.3 \%$ and $90.8 \%$ for cyan, magenta, and yellow inkjets. However, fewer than half (49.2\%) of the black inkjets were distinguishable. The poor discrimination is unsurprising since about half of the black inkjets had no detectable elements at all and the other half had only one detectable element (sulfur or potassium). Therefore, SEM-EDS is particularly limited for the analysis of black inkjets. For toner inks, SEM-EDS provided greater than $94 \%$ discrimination for each ink color. LA-ICP-MS provided greater than $98 \%$ discrimination for inkjets and greater than $97 \%$ discrimination for toners of the same color. The offset and intaglio samples included a wide variety of colors, making it impractical to evaluate the discrimination potential for inks of the same color. 
In order to assess the false exclusion rate, a set of duplicates and quality controls was analyzed for each ink type. For SEM-EDS, all duplicates were found to be indistinguishable from the original sample, providing $100 \%$ correct association for all ink types. For LA-ICP-MS, 100\% correct association was obtained for all inkjet, offset, and intaglio duplicates and controls. However, two toner duplicates were falsely excluded ( $92.3 \%$ correct association). These two excluded pairs were analyzed by different laser ablation units as part of an inter-instrumental variation assessment and only differed in intensity for one element $(\mathrm{Mg})$.

The main advantages of using LA-ICP-MS for the analysis of printing inks are its superior sensitivity and faster analysis time. However, LA-ICP-MS is not currently routinely used in forensic laboratories because of its cost and its complexity. On the other hand, SEM-EDS is typically available in forensic laboratories. Moreover, the electron beam does not penetrate deeply into the sample, which is advantageous for the analysis of inks. SEM-EDS is generally thought of as a non-destructive technique, while LA-ICPMS is quasi-destructive, removing small $(\sim \mu \mathrm{g})$ amounts of the sample. Nevertheless, it should be noted that discoloration was observed for some inkjets, particularly the magenta inkjets.

Although LIBS was not originally included in the ink project, a small subset of toner and inkjet samples was subsequently analyzed with this technique. A total of 45 inkjet and 57 toner samples were analyzed. After analysis, sample pairs were initially compared using spectral overlay, as was the case for SEM-EDS and LA-ICP-MS. However, though spectral overlay provided good discrimination $(93.8 \%$ and $97.9 \%$ for inkjets and toners, respectively), the correct association results were poor even after normalizing the spectra 
( $78.9 \%$ and $21.7 \%$ for inkjets and toners, respectively). Thus, an alternative method for pairwise comparisons was evaluated. The peaks for all elements of interest were integrated and used to generate element ratios. All ink samples were then compared with a modified 4-sigma match criterion using the element ratios. The element ratios used for discrimination were selected based on their reproducibility and discrimination potential. If all ratios for a pair of ink samples were indistinguishable using the match criterion, then that pair was associated. However, if at least one ratio differed, then the pair was discriminated. The match criterion provided better results than the spectral overlay comparison. Inkjets showed $95.9 \%$ discrimination and $94.7 \%$ correct association. Moreover, $\sim 87 \%$ of the black inkjets were discriminated. Thus LIBS is more suitable than SEM-EDS for the analysis of black inkjets. A discrimination greater than $83.9 \%$ was observed for inkjets of the remaining three colors (cyan, magenta, and yellow). Toners showed $96.9 \%$ discrimination, slightly less than the $97.1 \%$ obtained with SEM-EDS, and $89.1 \%$ correct association. A discrimination greater than $95 \%$ was observed for all samecolor toner pairs. The LIBS results suggest that the technique is an attractive alternative to SEM-EDS or LA-ICP-MS. LIBS offers some of the same advantages as LA-ICP-MS (i.e. higher sensitivity than SEM-EDS and faster analysis) without the high cost or complexity. It is therefore more likely to be implemented in forensic laboratories than LA-ICP-MS. However, the poor reproducibility, as seen with the high false exclusion rate using spectral overlay, must be carefully taken into account when conducting pairwise comparisons.

The final part of the ink project involved the development of the Forensic Ink Analysis and Comparison System (FIACS). The searchable database uses PLSDA and KNN to 
compare an unknown ink sample to a reference collection. The overall goal of the FIACS database is to narrow down the possible sources for a questioned ink sample. To evaluate performance of the database, duplicate samples were treated as unknowns and compared to the reference samples. Overall, the superior discriminating power of LA-ICP-MS led to better performance than SEM-EDS. LA-ICP-MS provided a correct association $>94 \%$ for all ink types when both the PLSDA and KNN were used. However, it should be noted that the inkjet and intaglio duplicates set was quite limited (between 1 and 6 duplicates). A much larger set of duplicates is advisable in order to better estimate the correct classification rate of the FIACS database. SEM-EDS provided good association ( $\geq 95 \%)$ for the offset and intaglio set when PLSDA and KNN were combined. However, even after combining the two classification tools, SEM-EDS provided a correct association rate $\leq 76 \%$ for inkjet and toner samples. It is expected that a larger number of samples in the reference collection and the test set (i.e. duplicates) as well as a larger number of replicates per sample would improve the results since the algorithms would produce better models. For this reason, the FIACS database has the capability of being expanded as additional samples are collected and analyzed. There are several considerations that were not investigated for this project, but that nonetheless deserve attention. Firstly, all ink samples were collected over a short time period (a few months). It is currently unknown whether cartridges of the same manufacturer and model change in elemental composition over time. Secondly, pure color cartridges were collected and analyzed for the toner and inkjet set. Although black is the most common ink color encountered in questioned documents analysis, there may be cases in which a colored document is encountered. Since colored documents are printed by mixing the pure colors, the 
elemental composition of the color mixture would be a combination of the pure colors. Therefore, the FIACS database (which is populated only with pure colors) would not be able to correctly associate a color mixture to the individual colors. It may be feasible to isolate the contribution from each individual color; however, this was not investigated in the present study. Finally, paper contribution was not considered for the FIACS database. Currently, it is unknown whether the same ink printed on different paper would be correctly associated using the FIACS database. Paper contribution may not be an issue for the inks with a thick film (toner and intaglio), but is expected to be problematic for inks with a thin film (inkjet and offset). As in the case for color mixtures, it may be possible to isolate the contribution from the ink and paper. Further research is necessary to address these concerns.

The second project in this dissertation focused on the interpretation of forensic evidence. Although the methods discussed can be applied to many types of forensic evidence, this study focused on glass evidence since it is a well-characterized matrix. Typically, forensic evidence is compared using a match criterion, much like what was described previously for the analysis of inks using LIBS. Known and questioned samples that differ in the concentration of one or more element(s) are excluded. However, if no differences are found, then the known sample cannot be excluded as a possible source for the questioned sample. A verbal scale may be used to provide a level of association in the case where the known and questioned samples are indistinguishable. The match criterion, referred to as the frequentist approach, has several drawbacks: it suffers from the "fall off the cliff" effect, it does not take the rarity of the elemental profile into account, and it does not provide a weight of evidence. These disadvantages can be overcome using a 
likelihood ratio (LR) approach to evidence interpretation. The LR is defined as the probability of the evidence given $\mathrm{H}_{1}$ (an association) divided by the probability of the evidence given $\mathrm{H}_{2}$ (a non-association). A LR greater than 1 shows support for $\mathrm{H}_{1}$ and a LR less than 1 shows support for $\mathrm{H}_{2}$. Furthermore, a larger LR offers stronger support for $\mathrm{H}_{1}$ and a smaller LR offers stronger support for $\mathrm{H}_{2}$. For the calculation of a LR, a relevant background population is necessary. Two databases were used in this study: the LA-ICPMS FIU vehicle database, which consists of windshield glass samples from 210 vehicles; and the LA-ICP-MS BKA casework database, which consists of different types of glass samples encountered in casework. The LR was calculated for each database using the Multivariate Kernel (MVK) model, which resulted in extreme values. Thus, in order to limit the LR to more reasonable values, the Pool Adjacent Violators (PAV) algorithm was used to calibrate the LR. A double 10-fold cross-validation procedure was implemented. Compared to the match criterion, the calibrated LR offered improved false exclusion rates $(<1.2 \%)$ and similar false inclusion rates $(<1 \%)$. Moreover, the majority of the misleading evidence (i.e. false exclusions and inclusions) provided weak support for the incorrect hypothesis; that is, the LR values were close to one. Finally, samples that showed incorrect strong associations originated from vehicles of the same make and/or model.

After these initial results, it was decided that hafnium should be excluded from the element menu since it is highly correlated with zirconium and does not provide additional discrimination. Furthermore, several samples were removed from the FIU and BKA database: for the FIU database, outer pane samples that were indistinguishable from the inner pane of the same vehicle were excluded in order to avoid biasing the database; for 
the BKA database, unusual glass samples, suspected of being non-float glass, were removed, again in order to avoid biasing the database. The LR was then recalculated using these limited databases. Overall, the BKA database performed better than the FIU database (false inclusion and false exclusion rate $<0.54 \%$ ). Therefore, the limited BKA database was selected as the background population in order to calculate a calibrated likelihood ratio for several smaller LA-ICP-MS glass datasets.

The "BKA same pane" set consisted of 34 glass fragments, analyzed 44 times, that all originated from the same source. Therefore, all $946(44 \times 43 \div 2=946)$ pairwise comparisons should be associated. The calibrated LR resulted in no false exclusions for this dataset. The "BKA float glass" set included 62 float glass samples from different sources. A total of 6 out of 1,891 pairwise comparisons were falsely included using the calibrated LR $(0.32 \%$ false inclusion rate). In all cases, the pairs that were falsely included were manufactured by the same glass company (Pilkington or Saint Gobain). The "FBI vehicle glass" set included 151 glass fragments from windshields, back windows, and side windows of automobiles. A slightly high false inclusion rate $(2.6 \%)$ was observed for this set. However, many glass samples originated from different windows of the same vehicle and therefore may have originated from the same glass source. A total of 87 out of the 245 falsely included pairs originated from a different vehicle make and/or year of manufacture. The "Guardia Civil vehicle glass" set consisted of 38 glass fragments from windshields, back windows, and side windows of automobiles. Only 6 pairs were falsely included $(0.63 \%)$, four of which originated from the same vehicle. The "BKA container glass" set included 20 samples from glass containers. A slightly high false inclusion rate (2.1\%) was observed for this set as well. 
However, this was a fairly small sample set. Moreover, since the BKA casework database used as the background population consists mostly of float (not container) glass, it is suspected that the BKA casework database is an inappropriate background database. The last dataset was the most interesting: the "NFI daily samples" set consisted of 41 glass samples, one from each day of production over the course of 41 days in the same glass manufacturing plant. Since the elemental composition is known to vary over time within the same manufacturing plant, it was expected that samples produced closer in time should be fairly similar, while samples produced further apart in time should be more dissimilar. The majority of the glass pairs that were manufactured only one day apart were strongly associated ( $L R>1000)$. As the number of days between the production of two glass samples increased, fewer pairs were strongly associated and more pairs were either weakly associated or excluded. Thus, the number of strong associations and the number of days between production dates showed a negative correlation. On the other hand, the number of strong exclusions and the number of days between production dates showed a positive correlation.

The final part of the glass project included an inter-laboratory study: the Glass Interpretation Working Group (GIWG). A total of 10 LA-ICP-MS laboratories participated in three exercises. A calibrated LR was calculated for all known and questioned sample comparison pairs using three background databases: the limited BKA casework database, the limited FIU vehicle database, and the combined limited BKA and FIU database. No false inclusions were observed for any different-source pairs and false exclusion rates $<6 \%$ were observed for same-source pairs. Although the different background databases resulted in the same correct association rates (>94\%), the BKA 
database performed best in terms of the weight of evidence. That is, same-source pairs were more strongly associated for the BKA database than for the remaining two databases. This is likely because the BKA casework database exhibits greater variation between sources than the FIU vehicle database (which includes many samples from similar vehicles). Some labs exhibited a weak association for same-source pairs, even when the BKA database was used as the background population. These labs were found to have reproducibility issues for specific elements. Thus the LR can provide a measure of uncertainty for an association (or exclusion). That is, samples with high RSDs result in a limited LR.

Overall, the calibrated likelihood ratio performed well for the double 10-fold cross validation experiments, for the smaller independent datasets, and for the inter-laboratory data. Moreover, it is clear that the LR provides several advantages over the match criterion: it provides a quantitative measure for the weight of evidence, it does not suffer from the "fall off the cliff" effect, and it takes the rarity of the elemental profile into account. Still, the results indicate that the selection of the background database is an important step for the calculation of the LR. A disadvantage of the LR approach is that many forensic laboratories do not have a database available. It may be possible for forensic glass analysts to use a single compiled database for the calculation of the LR. A shared database may not be the most ideal approach since the frequency of glass elemental profiles is expected to differ across different locations and thus may not be representative of the relevant population. However, this study showed that regardless of the database used, good correct association rates (>94\%) and no false inclusions were obtained, thus the use of a shared database may be justified. A second disadvantage of the 
LR approach is its complexity. An exit survey was sent to all inter-laboratory participants in order to gauge their reactions to the LR approach, which is a relatively new approach in the United States. Several participants stated that the following would hinder their use of the LR approach: the complexity of the calculations, the difficulty in interpreting the LR value, the difficulty in explaining the LR in court, and the opinion that the current method (i.e., the match criterion) is appropriate and the LR is unnecessary. Therefore, before the LR is widely accepted in the forensic community, the analysts must be convinced of the improvement the LR offers over the currently used, and more subjective, interpretation approaches (e.g., verbal scale, frequency). The development of a user-friendly program for the calculation of the LR that is accessible to practitioners can potentially aid in encouraging analysts to become more familiar with the LR approach. Moreover, a standard methodology for the calculation of the LR is expected to increase the acceptance of the LR approach within the U.S. forensic community. 


\section{LIST OF REFERENCES}

1. Warner, R. D.; Adams II, R. M., Introduction to Security Printing. PIA/GATF Press: Pennsylvania, 2005.

2. Kobilinsky, L., Forensic Chemistry Handbook. John Wiley \& Sons: New Jersey, USA, 2012.

3. Saferstein, R., Forensic Science Handbook. 2 ed.; Prentice Hall: New Jersey, USA, 2002; Vol. 1.

4. Akao, Y.; Kobayashi, K.; Seki, Y., Examination of Spur Marks Found on Inkjetprinted Documents. Journal of forensic sciences 2005, 50 (4).

5. Jasuja, O. M. P.; Singla, A. K., Application of the ESDA in demonstrating traced forgeries. Forensic science international 1995, 75, 25-28.

6. Kaur, R.; Saini, K.; Sood, N. C., Application of Video Spectral Comparator (absorption spectra) for establishing the chronological order of intersecting printed strokes and writing pen strokes. Science \& Justice 2013, 53 (2), 212-219.

7. Saini, K.; Kaur, R.; Sood, N. C., A study for establishing the sequence of superimposed lines: inkjet versus writing instruments. Forensic science international 2009, 193 (1-3), 14-20.

8. Strach, S. J.; McCormack, G. M.; Radley, R. W.; Westwood, P. D., Secondary impressions of writing detected by ESDA. Forensic science international 1995, 74, 193204.

9. Sugawara, S., Comparison of Near Infrared Light Photography and Middle Infrared Light Photography for Deciphering Obliterated Writings. Journal of forensic sciences 2004, 49 (6), 1349-1352.

10. Aginsky, V. N., Forensic Examination of "Slightly Soluble" Ink Pigments Using Thin-Layer Chromatography. Journal of forensic sciences 1993, 38 (5), 1131-1133.

11. Aginsky, V. N., Dating and Characterizing Writing, Stamp Pad and Jet Printer Inks by Gass $\backslash$ Chromatography/Mass Spectrometry. International Journal of Forensic Document Examiners 1996, 2 (2), 103-115. 
12. Armitage, S.; Saywell, S.; Roux, C.; Lennard, C.; Greenwood, P., The Analysis of Forensic Samples Using Laser Micro-Pyrolysis Gas Chromatography Mass Spectrometry. Journal of forensic sciences 2001, 46 (5), 1043-1052.

13. Assis, A. C.; Barbosa, M. F.; Nabais, J. M.; Custodio, A. F.; Tropecelo, P., Diamond cell Fourier transform infrared spectroscopy transmittance analysis of black toners on questioned documents. Forensic science international 2012, 214, 59-66.

14. Braz, A.; Lopez-Lopez, M.; Garcia-Ruiz, C., Raman spectroscopy for forensic analysis of inks in questioned documents. Forensic science international 2013, 232, 20612.

15. Chu, P. C.; Cai, B. Y.; Tsoi, Y. K.; Yuen, R.; Leung, K. S.; Cheung, N. H., Forensic analysis of laser printed ink by X-ray fluorescence and laser-excited plume fluorescence. Analytical chemistry 2013, 85 (9), 4311-5.

16. Corzo, R.; Subedi, K.; Trejos, T.; Almirall, J., Evaluation of the Forensic Utility of Scanning Electron Microscopy-Energy Dispersive Spectroscopy and Laser AblationInductively Coupled Plasma-Mass Spectrometry for Printing Ink Examinations. Journal of forensic sciences 2016, 61 (3), 725-734.

17. Egan, W. J.; Galipo, R. C.; Kochanowski, B. K.; Morgan, S. L.; Bartick, E. G.; Miller, M. L.; Ward, D. C.; Mothershead, R. F., 2nd, Forensic discrimination of photocopy and printer toners. III. Multivariate statistics applied to scanning electron microscopy and pyrolysis gas chromatography/mass spectrometry. Analytical and bioanalytical chemistry 2003, 376 (8), 1286-1297.

18. Egan, W. J.; Morgan, S. L.; Bartick, E. G.; Merrill, R. A.; Taylor, H. J., 3rd, Forensic discrimination of photocopy and printer toners II. Discriminant analysis applied to infrared reflection-absorption spectroscopy. Analytical and bioanalytical chemistry 2003, 376 (8), 1279-85.

19. Heudt, L.; Debois, D.; Zimmerman, T. A.; Kohler, L.; Bano, F.; Partouche, F.; Duwez, A. S.; Gilbert, B.; De Pauw, E., Raman spectroscopy and laser desorption mass spectrometry for minimal destructive forensic analysis of black and color inkjet printed documents. Forensic science international 2012, 219, 64-75.

20. Johnson, C. E.; Martin, P.; Roberts, K. A.; Trejos, T.; Corzo, R.; Almirall, J. R.; Safer, A. M., The Capability of Raman Microspectroscopy to Differentiate Printing Inks. Journal of forensic sciences 2017, 66-79. 
21. Kemp, G. S.; Totty, R. N., The Differentiation of Toners Used in Photocopy Processes by Infrared Spectroscopy. Forensic science international 1983, 22, 75-83.

22. Kivioja, A.; Hartus, T.; Vuorinen, T.; Gane, P.; Jaaskelainen, A. S., Use of total internal reflection Raman (TIR) and attenuated total reflection infrared (ATR-IR) spectroscopy to analyze component separation in thin offset ink films after setting on coated paper surfaces. Applied spectroscopy 2013, 67 (6), 661-671.

23. Merrill, R. A.; Bartick, E. G.; Taylor, J. H., 3rd, Forensic discrimination of photocopy and printer toners I. The development of an infrared spectral library. Analytical and bioanalytical chemistry 2003, 376 (8), 1272-1278.

24. Brandi, J.; James, B. D.; Gutowski, S. J., Differentiation and Classification of Photocopier Toners. International Journal of Forensic Document Examiners 1997, 3, $324-344$.

25. Lennard, C. J.; Mazzella, W. D., A simple combined technique for the analysis of toners and adhesives. Journal of Forensic Science Society 1991, 365-371.

26. Pagano, L. W.; Surrency, M. J.; Cantu, A. A. Inks: Forensic Analysis by ThinLayer (Planar) Chromatography; 2000.

27. Poon, N. L.; Ho, S. S. H.; Li, C. K., Differentiation of coloured inks of inkjet printer cartridges by thin layer chromatography and high performance liquid chromatography. Science \& Justice 2005, 45 (4), 187-194.

28. Tandon, G.; Jasuja, O. P.; Sehgal, V. N., Thin layer chromatography analysis of photocopy toners. Forensic science international 1995, 73, 149-154.

29. Trejos, T.; Corzo, R.; Subedi, K.; Almirall, J., Characterization of toners and inkjets by laser ablation spectrochemical methods and Scanning Electron MicroscopyEnergy Dispersive X-ray Spectroscopy. Spectrochimica Acta Part B 2014, 92, 9-22.

30. Trzcinska, B. M., Classification of black powder toners on the basis of integrated analytical information provided by Fourier transform infrared spectrometry and X-ray fluorescence spectrometry. Journal of forensic sciences 2006, 51 (4), 919-924.

31. Trzcinska, B. M.; Brozek-Mucha, Z., The Possibilities of Identifying Photocopy Toners by Means of Infrared Spectroscopy (FT-IR) and Scanning Microscopy. Mikrochimica Acta 1997, 14, 235-237. 
32. Udriştioiu, E. G.; Bunaciu, A. A.; Aboul-Enein, H. Y.; Tănase, I. G., Forensic Analysis of Color Toners by Raman Spectroscopy. Instrumentation Science \& Technology 2009, 37 (1), 23-29.

33. Williams, R. L., Analysis of Photocopying Toners by Infrared Spectroscopy. Forensic science international 1983, 22, 85-95.

34. Szynkowska, M. I.; Czerski, K.; Paryjczak, T.; Parczewski, A., Ablative analysis of black and colored toners using LA-ICP-TOF-MS for the forensic discrimination of photocopy and printer toners. Surface and Interface Analysis 2010, 42 (5), 429-437.

35. Subedi, K.; Trejos, T.; Almirall, J., Forensic analysis of printing inks using tandem Laser Induced Breakdown Spectroscopy and Laser Ablation Inductively Coupled Plasma Mass Spectrometry. Spectrochimica Acta Part B: Atomic Spectroscopy 2015, 103-104, 76-83.

36. Trejos, T.; Koons, R.; Weis, P.; Becker, S.; Berman, T.; Dalpe, C.; Duecking, M.; Buscaglia, J.; Eckert-Lumsdon, T.; Ernst, T.; Hanlon, C.; Heydon, A.; Mooney, K.; Nelson, R.; Olsson, K.; Schenk, E.; Palenik, C.; Pollock, E. C.; Rudell, D.; Ryland, S.; Tarifa, A.; Valadez, M.; van Es, A.; Zdanowicz, V.; Almirall, J., Forensic analysis of glass by $\mu$-XRF, SN-ICP-MS, LA-ICP-MS and LA-ICP-OES: evaluation of the performance of different criteria for comparing elemental composition. Journal of Analytical Atomic Spectrometry 2013, 28 (8), 1270-1282.

37. Weis, P.; Dücking, M.; Watzke, P.; Menges, S.; Becker, S., Establishing a match criterion in forensic comparison analysis of float glass using laser ablation inductively coupled plasma mass spectrometry. Journal of Analytical Atomic Spectrometry 2011, 26 (6), 1273-1284.

38. Koons, R. D.; Buscaglia, J., Interpretation of Glass Composition Measurements: The Effects of Match Criteria on Discrimination Capability. Journal of forensic sciences 2002, 47 (3), 505-512.

39. Curran, J. M.; Triggs, C. M.; Almirall, J. R.; Buckleton, J. S.; Walsh, K. A. J., The interpretation of elemental composition measurements from forensic glass evidence: II. Science \& Justice 1997, 37 (4), 245-249.

40. Aitken, C. G. G.; Lucy, D., Evaluation of trace evidence in the form of multivariate data. Journal of Applied Statistics 2004, 53, 109-122. 
41. Robertson, B.; Vignaux, G. A.; Berger, C. E. H., Interpreting Evidence: Evaluating Forensic Science in the Courtroom. 2 ed.; John Wiley \& Sons, Ltd: West Sussex, United Kingdom, 2016.

42. Zadora, G.; Martyna, A.; Ramos, D.; Aitken, C., Statistical Aalysis in Forensic Science: Evidential Value of Multivariate Physicochemical Data. John Wiley \& Sons, Ltd: West Sussex, United Kingdom, 2014.

43. Zadora, G., Evaluation of evidence value of glass fragments by likelihood ratio and Bayesian Network approaches. Analytica chimica acta 2009, 642 (1-2), 279-290.

44. van Es, A.; Wiarda, W.; Hordijk, M.; Alberink, I.; Vergeer, P., Implementation and assessment of a likelihood ratio approach for the evaluation of LA-ICP-MS evidence in forensic glass analysis. Science \& Justice 2017, 57 (3), 181-192.

45. Vergeer, P.; van Es, A.; de Jongh, A.; Alberink, I.; Stoel, R., Numerical likelihood ratios outputted by LR systems are often based on extrapolation: When to stop extrapolating? Science \& Justice 2016, 56 (6), 482-491.

46. Corzo, R.; Hoffman, T.; Weis, P.; Franco-Pedroso, J.; Ramos, D.; Almirall, J., The Use of LA-ICP-MS Databases to Estimate Likelihood Ratios for the Forensic Analysis of Glass Evidence. Talanta 2018, https://doi.org/10.1016/j.talanta.2018.02.027.

47. Biedermann, A.; Bozza, S.; Taroni, F., Probabilistic evidential assessment of gunshot residue particle evidence (Part I): likelihood ratio calculation and case preassessment using Bayesian networks. Forensic science international 2009, 191 (1-3), 2435.

48. Biedermann, A.; Bozza, S.; Taroni, F., Probabilistic evidential assessment of gunshot residue particle evidence (Part II): Bayesian parameter estimation for experimental count data. Forensic science international 2011, 206 (1-3), 103-10.

49. Champod, C.; Taroni, F., Bayesian framework for the evaluation of fibre transfer evidence. Science \& Justice 1997, 37 (2), 75-83.

50. Causin, V.; Schiavone, S.; Marigo, A.; Carresi, P., Bayesian framework for the evaluation of fiber evidence in a double murder--a case report. Forensic science international 2004, 141 (2-3), 159-70.

51. Collins, A.; Morton, N. E., Likelihood ratios for DNA identification. Proceedings of the National Academy of Sciences of the United States of America 1994, 91, 60076011. 
52. Biedermann, A.; Taroni, F., Bayesian networks for evaluating forensic DNA profiling evidence: a review and guide to literature. Forensic Science International: Genetics 2012, 6 (2), 147-157.

53. Curran, J. M.; Buckleton, J. S., An investigation into the performance of methods for adjusting for sampling uncertainty in DNA likelihood ratio calculations. Forensic Science International: Genetics 2011, 5 (5), 512-516.

54. Curran, J. M.; Buckleton, J.; Triggs, C. M., The robustness of a continuous likelihood approach to bayesian analysis of forensic glass evidence. Forensic science international 1999, 104, 91-103.

55. Bolck, A.; Weyermann, C.; Dujourdy, L.; Esseiva, P.; van den Berg, J., Different likelihood ratio approaches to evaluate the strength of evidence of MDMA tablet comparisons. Forensic science international 2009, 191 (1-3), 42-51.

56. Bolck, A.; Ni, H.; Lopatka, M., Evaluating score- and feature-based likelihood ratio models for multivariate continuous data: applied to forensic MDMA comparison. Law, Probability and Risk 2015, 14 (3), 243-266.

57. Bolck, A.; Alberink, I., Variation in likelihood ratios for forensic evidence evaluation of XTC tablets comparison. Journal of Chemometrics 2011, 25 (1), 41-49.

58. Farmer, N.; Meier-Augenstein, W.; Lucy, D., Stable isotope analysis of white paints and likelihood ratios. Science \& Justice 2009, 49 (2), 114-9.

59. Franco-Pedroso, J.; Ramos, D.; Gonzalez-Rodriguez, J., Gaussian Mixture Models of Between-Source Variation for Likelihood Ratio Computation from Multivariate Data. PLoS One 2016, 11 (2), e0149958.

60. Frost, D.; Ishihara, S. In Likelihood Ratio-based Forensic Voice Comparison on L2 speakers: A Case of Hong Kong native male production of English vowels, Australasian Language Technology Association Workshop, 2015; pp 39-47.

61. Gonzalez-Rodriguez, J.; Drygajlo, A.; Ramos-Castro, D.; Garcia-Gomar, M.; Ortega-Garcia, J., Robust estimation, interpretation and assessment of likelihood ratios in forensic speaker recognition. Computer Speech \& Language 2006, 20 (2-3), 331-355.

62. van Leeuwen, D. A.; Brümmer, N., The distribution of calibrated likelihood-ratios in speaker recognition. Interspeech 2013. 
63. Martyna, A.; Sjastad, K.-E.; Grzegorz, Z.; Ramos, D., Analysis of lead isotopic ratios of glass objects with the aim of comparing them for forensic purposes. Talanta 2013, 105, 158-166.

64. Michalska, A.; Martyna, A.; Zięba-Palus, J.; Zadora, G., Application of a likelihood ratio approach in solving a comparison problem of Raman spectra recorded for blue automotive paints. Journal of Raman Spectroscopy 2015, 46 (9), 772-783.

65. Menzyk, A.; Martyna, A.; Zadora, G., Evidential value of polymeric materialschemometric tactics for spectral data compression combined with likelihood ratio approach. The Analyst 2017, 142 (20), 3867-3888.

66. Martyna, A.; Zadora, G.; Stanimirova, I.; Ramos, D., Wine authenticity verification as a forensic problem: an application of likelihood ratio test to label verification. Food chemistry 2014, 150, 287-295.

67. Muehlethaler, C.; Massonnet, G.; Hicks, T., Evaluation of infrared spectra analyses using a likelihood ratio approach: A practical example of spray paint examination. Science \& Justice 2016, 56 (2), 61-72.

68. Ramos, D.; Haraksim, R.; Meuwly, D., Likelihood ratio data to report the validation of a forensic fingerprint evaluation method. Data Brief 2017, 10, 75-92.

69. Trejos, T.; Almirall, J. R., Sampling strategies for the analysis of glass fragments by LA-ICP-MS Part I. Micro-homogeneity study of glass and its application to the interpretation of forensic evidence. Talanta 2005, 67 (2), 388-95.

70. Trejos, T.; Koons, R.; Becker, S.; Berman, T.; Buscaglia, J.; Duecking, M.; Eckert-Lumsdon, T.; Ernst, T.; Hanlon, C.; Heydon, A.; Mooney, K.; Nelson, R.; Olsson, K.; Palenik, C.; Pollock, E. C.; Rudell, D.; Ryland, S.; Tarifa, A.; Valadez, M.; Weis, P.; Almirall, J., Cross-validation and evaluation of the performance of methods for the elemental analysis of forensic glass by $\mu-\mathrm{XRF}, \mathrm{ICP}-\mathrm{MS}$, and LA-ICP-MS. Analytical and bioanalytical chemistry 2013, 405 (16), 5393-409.

71. Trejos, T.; Almirall, J. R., Sampling strategies for the analysis of glass fragments by LA-ICP-MS Part II: Sample size and sample shape considerations. Talanta 2005, 67 (2), 396-401.

72. Trejos, T.; Montero, S.; Almirall, J. R., Analysis and comparison of glass fragments by laser ablation inductively coupled plasma mass spectrometry (LA-ICP-MS) and ICP-MS. Analytical and bioanalytical chemistry 2003, 376 (8), 1255-64. 
73. Latkoczy, C.; Becker, S.; Ducking, M.; Gunther, D.; Hoogewerff, J. A.; Almirall, J. R.; Buscaglia, J.; Dobney, A.; Koons, R. D.; Montero, S.; Peijl, G. J. Q. v. d.; Stoecklein, W. R. S.; Trejos, T.; Watling, J. R.; Zdanowicz, V. S., Development and Evaluation of a Standard Method for the Quantitative Determination of Elements in Float Glass Samples by LA-ICP-MS. Journal of forensic sciences 2005, 50 (6), 1327-1341.

74. Leach, R. H.; Pierce, R. J., The Printing Ink Manual. 5 ed.; Springer: The Netherlands, 1993.

75. Harris, D. C., Quantitative Chemical Analysis. 7 ed.; W. H. Freeman and Company: New York, NY, 2007.

76. AuClair, C. J.; Lu, C. H. Colored toner and developer composition. US 4410617 A, 1983.

77. Bruinsma, P. J. Addition of copper salts and copper complexes to thermal inkjet inks for kogation reduction. US 6616273 B1, 2003.

78. Carlini, R.; Sacripante, G. G.; Veregin, R. P. N. Toner compositions. US 6143457 A, 2000.

79. Fujikawa, H.; Hashimoto, A.; Hayami, K.; Ida, T.; Komatsu, N.; Tanikawa, H. Color Toner. EP 1467258 A2, 2004.

80. Gibson, G. A.; Harrington, R. J.; Thomas, M. T. Charge control agent combination for a liquid toner. US 5200289 A, 1993.

81. Goodbrand, H. B.; Ong, B. S. Toner compositions with zinc and boron charge enhancing additives. US 5538829 A, 1996.

82. Grande, M. L.; Grushkin, B.; Young, E. F.; Lundy, D. A.; Matalevich, J. R. E. Magnetic toner compositions with silica, strontium titanate and polyvinylidene fluoride. US 5486443 A, 1996.

83. Grigoryan, L. S.; Yakushi, K. Magnetic composition, magnetic toner and ink containing the magnetic composition. US 5506079 A, 1996.

84. Gruber, R. J.; Haack, J. L.; Hsieh, B. R. Toner compositions with organo boron negative charge enhancing additives. US 4898802 A, 1990.

85. Herget, G.; Hechler, W.; Husseini, B.; Mohr, H.; Smolka, R. Offset printing ink. US 5445671 A, 1995. 
86. Herrmann, H.-F.; Hohner, G. Method of using polyolefin waxes. US 6080902 A, 2000.

87. Hirata, N.; Okamoto, H.; Zama, Y.; Nakamura, M.; Yanagimoto, T.; Yamada, H.; Tsuchiya, M. Pigment. US 20080096999 A1, 2008.

88. Kasperchik, V. Oxide pigment dispersion for inkjet ink. EP 2630199 A1, 2013.

89. Kwarta, M. S.; Helenbrook, H. J.; Spence, J. M. Electrophotographic toner and developer compositions. EP 0282740 A2, 1988.

90. Lefebvre, O.; Schaller, C.; Degott, P.; Müller, E. Vanadium-drier intaglio ink. US 8807036 B2, 2014.

91. Lehmann, U.; Frick, M. Magenta inks comprising copper complex azo dyes based on 1-naphthol-di- or tri-sulfonic acids. US 6521032 B1, 2003.

92. Lehmann, U.; Stadler, U. L.; Mamak, M.; Knischka, R. Tungsten oxides used to increase the heat- input amount of near infrared radiation. EP 2244879 A2, 2010.

93. Lewis, D. M.; Broadbent, P. J. Security inks containing infrared absorbing metal compounds. US 8157905 B2, 2012.

94. Martin, T. W.; Bugner, D. E. Pigmented inkjet inks containing aluminum stabilized colloidal silica. US 5925178 A, 1999.

95. Mcgorrin, M. Ink-jet ink formulations containing magnesium sulfate. EP 2118212 A2, 2009.

96. Nomura, M.; Nishiyama, K.; Kasai, M.; Ishii, H. Drier for printing ink and printing ink containing drier. US 20040040468 A1, 2004.

97. Park, B.-h.; Ha, Y.-u. Inkjet printhead having conductive epoxy resin. US 20070060675 A1, 2007.

98. Patel, R. D.; Sacripante, G. G. Toner processes using sodium sulfonated polyester resins. US 5593807 A, 1997.

99. Pickering, T. R. Toner composition having coated strontium titanate additive. US 20070281233 A1, 2007. 
100. Povirk, C. A.; Thompson, R. J.; Winfield, M. J. Color toner. US 5998079 A, 1999.

101. Raue, R.; Psaar, H. Dry toners containing fanal pigments based on cationic dyes. US 4869989 A, 1989.

102. Sacripante, G. G.; Farrugia, V. M.; Hawkins, M. S. Toner. US 6824944 B2, 2004.

103. Sugamo, H.; Chuo-ku, T. Aqueous Pigment Paste for Offset Ink, Process for Producing the Paste, and Use Thereof. EP 0784086 A1, 1997.

104. Vonwiller, S. C.; Indusegaram, S.; Gonzaga, G.; Ridley, D. D.; Silverbrook, K. Inkjet dyes exhibiting reduced kogation. US 8029611 B2, 2011.

105. Yeh, A.-G.; Valentini, J. E. Inkjet inks with increased optical density. US 6899754 B2, 2005.

106. Zhen Lai, Y. T.; Cheng, C.-M. Toner compositions. US 8080353 B2, 2011.

107. Zou, W. K.; Zhu, L.; Zhan, X.; Xiao, F.; Wang, X. Continuous ink jet printing ink composition. US 6726756 B1, 2004.

108. Adel, J.; Czech, E. Finely divided blue molybdenum oxide. US 5512264 A, 1996.

109. HAO, H.-Y.; Du, B.-C.; Zhang, C.-H.; Fan, S.-S. Inkjet ink and method for making the same. US 8623938 B2, 2014.

110. Gilmour, C. L., A Comparison of Laser Printed and Photocopied Documents Can They Be Distinguished? Can Soc Forens Sci J 1994, 27 (4), 245-259.

111. Lennard, C.; El-Deftar, M. M.; Robertson, J., Forensic application of laserinduced breakdown spectroscopy for the discrimination of questioned documents. Forensic science international 2015, 254, 68-79.

112. Curran, J.; Hicks, T.; Buckleton, J., Forensic Interpretation of Glass Evidence. CRC Press: Boca Raton, Florida, USA, 2000.

113. Pitts, K.; Trejos, T.; Watling, J. R.; Almirall, J., A guide for the quantitative elemental analysis of glass using laser ablation inductively coupled plasma mass spectrometry. Atomic Spectroscopy 2006, 27 (3), 69-75. 
114. ASTM E2926-13: Standard Test Method for Forensic Comparison of Glass Using Micro X-ray Fluorescence ( $\mu$-XRF) Spectrometry. ASTM International: West Conshohocken, PA, 2013.

115. ASTM E2927-13: Standard Test Method for Determination of Trace Elements in Soda-Lime Glass Samples Using Laser Ablation Inductively Coupled Plasma Mass Spectrometry for Forensic Comparisons. ASTM International: West Conshohocken, PA, 2013.

116. Bridge, C. M.; Powell, J.; Steele, K. L.; Sigman, M. E., Forensic comparative glass analysis by laser-induced breakdown spectroscopy. Spectrochimica Acta Part B: Atomic Spectroscopy 2007, 62 (12), 1419-1425.

117. Bridge, C. M.; Powell, J.; Steele, K. L.; Williams, M.; MacInnis, J. M.; Sigman, M. E., Characterization of Automobile Float Glass with Laser-Induced Breakdown Spectroscopy and Laser Ablation Inductively Coupled Plasma Mass Spectrometry. Applied spectroscopy 2006, 60 (10), 1181-1187.

118. McIntee, E.; Viglino, E.; Kumor, S.; Rinke, C.; Ni, L.; Sigman, M. E., Nonparametric permutation test for the discrimination of float glass samples based on LIBS spectra. Journal of Chemometrics 2010, 24, 312-319.

119. Naes, B. E.; Umpierrez, S.; Ryland, S.; Barnett, C.; Almirall, J. R., A comparison of laser ablation inductively coupled plasma mass spectrometry, micro X-ray fluorescence spectroscopy, and laser induced breakdown spectroscopy for the discrimination of automotive glass. Spectrochimica Acta Part B: Atomic Spectroscopy 2008, 63 (10), 1145-1150.

120. Rodriguez-Celis, E. M.; Gornushkin, I. B.; Heitmann, U. M.; Almirall, J. R.; Smith, B. W.; Winefordner, J. D.; Omenetto, N., Laser induced breakdown spectroscopy as a tool for discrimination of glass for forensic applications. Analytical and bioanalytical chemistry 2008, 391 (5), 1961-8.

121. Goldstein, J.; Newbury, D.; Joy, D.; Lyman, C.; Echlin, P.; Lifshin, E.; Sawyer, L.; Michael, J., Scanning Electron Microscopy and X-Ray Microanalysis. 3 ed.; Springer: New York, 2003.

122. Musazzi, S.; Perini, U., Laser-Induced Breakdown Spectroscopy Theory and Applications. Springer: NY, USA, 2014.

123. Taylor, H. E., Inductively Coupled Plasma-Mass Spectrometry Practices and Techniques. Academic Press: San Diego, CA, 2001. 
124. Hill, S. J., Inductively Coupled Plasma Spectrometry and its Applications. Blackwell Publishing Ltd: Oxford, UK, 2007.

125. Sneddon, J.; Thiem, T. L.; Lee, Y.-I., Lasers in Analytical Atomic Spectroscopy. VCH Publishers, Inc.: New York, USA, 1997.

126. de Hoffmann, E.; Stroobant, V., Mass Spectrometry Principles and Applications. 3 ed.; John Wiley \& Sons Ltd.: West Sussex, England, 2007.

127. Miziolek, A. W.; Palleschi, V.; Schechter, I., Laser-Induced Breakdown Spectroscopy (LIBS) Fundamentals and Applications. Cambridge University Press: NY, USA, 2006.

128. Aragón, C.; Aguilera, J. A., Characterization of laser induced plasmas by optical emission spectroscopy: A review of experiments and methods. Spectrochimica Acta Part B: Atomic Spectroscopy 2008, 63 (9), 893-916.

129. Aguilera, J. A.; Aragón, C.; Cristoforetti, G.; Tognoni, E., Application of calibration-free laser-induced breakdown spectroscopy to radially resolved spectra from a copper-based alloy laser-induced plasma. Spectrochimica Acta Part B: Atomic Spectroscopy 2009, 64 (7), 685-689.

130. Cremers, D. A.; Radziemski, L. J., Handbook of Laser-Induced Breakdown Spectroscopy. 2 ed.; John Wiley \& Sons Ltd.: West Sussex, UK, 2013.

131. Singh, J. P.; Thakur, S. N., Laser-Induced Breakdown Spectroscopy. Elsevier: The Netherlands, 2007.

132. Hou, Z.; Wang, Z.; Lui, S.-1.; Yuan, T.; Li, L.; Li, Z.; Ni, W., Improving data stability and prediction accuracy in laser-induced breakdown spectroscopy by utilizing a combined atomic and ionic line algorithm. Journal of Analytical Atomic Spectrometry 2013, $28(1), 107-113$.

133. Ingle, J. D.; Crouch, S. R., Spectrochemical Analysis. Prentice Hall: New Jersey, USA, 1988.

134. ASTM E2330-12: Standard Test Method for Determination of Concentrations of Elements in Glass Samples Using Inductively Coupled Plasma Mass Spectrometry (ICPMS) for Forensic Comparisons. ASTM International: West Conshohocken, PA, 2012.

135. Adam, C., Essential Mathematics and Statistics for Forensic Science. John Wiley \& Sons Ltd.: West Sussex, UK, 2010. 
136. Lucy, D. R Package "comparison": Multivariate likelihood ratio calculation and evaluation, R Package Version 1.0-4; 2013.

137. Mair, P.; Leeuw, J. D.; Hornik, K. R Package "isotone": Active Set and Generalized PAVA for Isotone Optimization, R Package Version 1.1-0; 2015.

138. Mullen, K. M.; Stokkum, I. H. M. v. R Package "nnls": The Lawson-Hanson algorithm for non-negative least squares (NNLS), R Package Version 1.4; 2012.

139. Ramos, D.; Gonzalez-Rodriguez, J., Reliable support: Measuring calibration of likelihood ratios. Forensic science international 2013, 230 (1-3), 156-169.

140. Brümmer, N.; du Preez, J., Application-independent evaluation of speaker detection. Computer Speech \& Language 2006, 20 (2-3), 230-275.

141. Zadora, G.; Ramos, D., Evaluation of glass samples for forensic purposes - An application of likelihood ratios and an information-theoretical approach. Chemometrics and Intelligent Laboratory Systems 2010, 102 (2), 63-83.

142. Ramos, D.; Gonzalez-Rodriguez, J.; Zadora, G.; Aitken, C., Informationtheoretical assessment of the performance of likelihood ratio computation methods. Journal of forensic sciences 2013, 58 (6), 1503-18.

143. Magdassi, S., The Chemistry of Inkjets. World Scientific Publishing: New Jersey, 2010.

144. May, T. W.; Wiedmeyer, R. H., A Table of Polyatomic Interferences in ICP-MS. Atomic Spectroscopy 1998, 19 (5), 150-155.

145. Trejos, T.; Torrione, P.; Corzo, R.; Raeva, A.; Subedi, K.; Williamson, R.; Yoo, J. H.; Almirall, J., A novel forensic tool for the characterization and comparison of printing ink evidence: development and evaluation of a searchable database using data fusion of spectrochemical methods. Journal of forensic sciences 2016, 61 (3), 715-724.

146. Miller, J. N.; Miller, J. C., Statistics and Chemometrics for Analytical Chemistry. 6 ed.; Pearson: England, UK, 2010.

147. Jones, B.; Nachtsheim, C. J., A Class of Three-Level Designs for Definitive Screening in the Presence of Second-Order Effects. Journal of Quality Technology 2011, $43(1), 1-15$. 
148. Jones, B.; Nachtsheim, C. J., Definitive Screening Designs with Added TwoLevel Categorical Factors. Journal of Quality Technology 2013, 45 (2), 121-129.

149. Baudelet, M., Laser Spectroscopy for Sensing: Fundamentals, Techniques and Applications. Woodhead Publishing: Sawston, UK, 2014.

150. Meuwly, D.; Ramos, D.; Haraksim, R., A guideline for the validation of likelihood ratio methods used for forensic evidence evaluation. Forensic science international 2017, 276, 142-153. 


\section{APPENDICES}


Appendix A

Inkjet Collection

\begin{tabular}{|c|c|c|c|c|}
\hline Sample ID & Brand & Color & Model & Manufacturer \# \\
\hline IJ 01 K BR & Brother & Black & LC61BK & LC61BK \\
\hline IJ 02 K CN & Canon & Black & 210 & PG210 \\
\hline IJ 03 K CN & Canon & Black & 50 & PG-50 \\
\hline IJ 04 Y CN & Canon & Yellow Tri-Color & 51 & CL-51 \\
\hline IJ 05 M CN & Canon & Magenta Tri-Color & 51 & CL-51 \\
\hline IJ 06 C CN & Canon & Cyan Tri-Color & 51 & CL-51 \\
\hline IJ 07 K ЕP & Epson & Black & $676 x 1$ & $676 x 1$ \\
\hline IJ 08 C EP & Epson & Cyan & $676 x 1$ & $676 x 1$ \\
\hline IJ 09 М EP & Epson & Magenta & $676 x 1$ & $676 \times 1$ \\
\hline IJ 10 Y EP & Epson & Yellow & $676 x 1$ & $676 x 1$ \\
\hline IJ 11 K EP & Epson & Black & T032120 & C13T032120 \\
\hline IJ 12 K HP & $\begin{array}{l}\text { Hewlett } \\
\text { Packard }\end{array}$ & Black & 564 & CB316WN \\
\hline IJ 13 K HP & $\begin{array}{l}\text { Hewlett } \\
\text { Packard }\end{array}$ & Black & 940 & C4902AN \\
\hline IJ 14 K HP & $\begin{array}{l}\text { Hewlett } \\
\text { Packard }\end{array}$ & Black & 60 & CC640W \\
\hline IJ 15 K HP & $\begin{array}{l}\text { Hewlett } \\
\text { Packard }\end{array}$ & Black & $901 \times 1$ & CC654A \\
\hline IJ 16 K HP & $\begin{array}{l}\text { Hewlett } \\
\text { Packard }\end{array}$ & Black & 74 & CB335WN \\
\hline IJ 17 K HP & $\begin{array}{l}\text { Hewlett } \\
\text { Packard }\end{array}$ & Black & 60 & CC640W \\
\hline IJ 18 K HP & $\begin{array}{l}\text { Hewlett } \\
\text { Packard }\end{array}$ & Black & 61 & CH561WN \\
\hline IJ 19 K HP & $\begin{array}{l}\text { Hewlett } \\
\text { Packard }\end{array}$ & Black & $901 x 1$ & CC654A \\
\hline IJ 20 K HP & $\begin{array}{l}\text { Hewlett } \\
\text { Packard }\end{array}$ & Black & $60 \times 1$ & CC641W \\
\hline IJ 21 C HP & $\begin{array}{l}\text { Hewlett } \\
\text { Packard }\end{array}$ & Cyan Tri-Color & $60 \times 1$ & CC644W \\
\hline IJ 22 M HP & $\begin{array}{l}\text { Hewlett } \\
\text { Packard }\end{array}$ & Magenta Tri-Color & $60 \times 1$ & CC644W \\
\hline IJ 23 Y HP & $\begin{array}{l}\text { Hewlett } \\
\text { Packard }\end{array}$ & Yellow Tri-Color & $60 \times 1$ & CC644W \\
\hline IJ 24 K HP & $\begin{array}{l}\text { Hewlett } \\
\text { Packard }\end{array}$ & Black & 45 & $51645 \mathrm{~A}$ \\
\hline IJ 25 C HP & $\begin{array}{l}\text { Hewlett } \\
\text { Packard }\end{array}$ & Cyan Tri-Color & 78 & C6578D \\
\hline
\end{tabular}




\begin{tabular}{|c|c|c|c|c|}
\hline Sample ID & Brand & Color & Model & Manufacturer \# \\
\hline IJ 26 M HP & $\begin{array}{l}\text { Hewlett } \\
\text { Packard }\end{array}$ & Magenta Tri-Color & 78 & C6578D \\
\hline IJ 27 Y HP & $\begin{array}{l}\text { Hewlett } \\
\text { Packard }\end{array}$ & Yellow Tri-Color & 78 & C6578D \\
\hline IJ 28 K LX & Lexmark & Black & 28 & $18 \mathrm{C} 1428$ \\
\hline IJ 29 K LX & Lexmark & Black & $44 \times 1$ & $18 Y 0144$ \\
\hline IJ 30 C LX & Lexmark & Cyan Tri-Color & $43 \mathrm{xl}$ & $18 Y 0143$ \\
\hline IJ 31 M LX & Lexmark & Magenta Tri-Color & $43 \mathrm{xl}$ & $18 Y 0143$ \\
\hline IJ 32 Y LX & Lexmark & Yellow Tri-Color & $43 \mathrm{xl}$ & $18 Y 0143$ \\
\hline IJ 33 K LX & Lexmark & Black & 17 & 10N0595 \\
\hline IJ 34 C LX & Lexmark & Cyan Tri-Color & 27 & 10N0595 \\
\hline IJ 35 M LX & Lexmark & Magenta Tri-Color & 27 & 10N0595 \\
\hline IJ 36 Y LX & Lexmark & Yellow Tri-Color & 27 & 10N0595 \\
\hline IJ 37 K CN & Canon & Black & $210 \mathrm{XL}$ & PG-210XL \\
\hline IJ 38 LM HP & $\begin{array}{l}\text { Hewlett } \\
\text { Packard }\end{array}$ & Light Magenta & 81 & $\mathrm{c} 4935 \mathrm{a}$ \\
\hline IJ 39 M HP & $\begin{array}{l}\text { Hewlett } \\
\text { Packard }\end{array}$ & Magenta & 80 & C4874A \\
\hline IJ 40 C HP & $\begin{array}{l}\text { Hewlett } \\
\text { Packard }\end{array}$ & Cyan & 80 & C4872A \\
\hline IJ 41 K HP & $\begin{array}{l}\text { Hewlett } \\
\text { Packard }\end{array}$ & Black & 80 & C4871A \\
\hline IJ 42 C OL & Olivetti & Cyan & N/A & N/A \\
\hline IJ 43 M OL & Olivetti & Magenta & N/A & N/A \\
\hline IJ 44 Y OL & Olivetti & Yellow & N/A & N/A \\
\hline IJ 45 C HP & $\begin{array}{l}\text { Hewlett } \\
\text { Packard }\end{array}$ & Cyan & $940 \mathrm{XL}$ & C4903A \\
\hline IJ 46 M HP & $\begin{array}{l}\text { Hewlett } \\
\text { Packard }\end{array}$ & Magenta & 940XL & C4904A \\
\hline IJ 47 K HP & $\begin{array}{l}\text { Hewlett } \\
\text { Packard }\end{array}$ & Black & 940XL & C4906A \\
\hline IJ 48 K BR & Brother & Black & LC79BK & LC79BK \\
\hline IJ 49 K SH & Sharp & Black & UX-C $80 B$ & $18 \mathrm{C} 1290$ \\
\hline IJ 50 K SH & Sharp & Black & UX-C70B & 10N0430 \\
\hline IJ 51 K CN & Canon & Black & 7 & PGI-7BK \\
\hline IJ 52 K CN & Canon & Black & 9 & PGI-9PBK \\
\hline IJ 53 C CN & Canon & Cyan & 9 & PGI-9C \\
\hline IJ 54 M CN & Canon & Magenta & 9 & PGI-9M \\
\hline IJ 55 Y CN & Canon & Yellow & 9 & PGI-9Y \\
\hline IJ 56 K KD & Kodak & Black & $10 \mathrm{~B}$ & 4K0035 \\
\hline IJ 57 K KD & Kodak & Black & 30 & $8 \mathrm{H} 3564$ \\
\hline IJ 58 C KD & Kodak & Cyan Tri-Color & 30 & $8 \mathrm{H} 3566$ \\
\hline
\end{tabular}




\begin{tabular}{|ccccc}
\hline Sample ID & Brand & Color & Model & Manufacturer \# \\
\hline IJ 59 M KD & Kodak & Magenta Tri-Color & 30 & $8 \mathrm{H} 3566$ \\
IJ 60 Y KD & Kodak & Yellow Tri-Color & 30 & $8 \mathrm{H} 3566$ \\
\hline IJ 61 K EP & Epson & Black & T034120 & C13T034120 \\
\hline IJ 62 LC EP & Epson & Light Cyan & T033520 & C13T033520 \\
IJ 63 M EP & Epson & Magenta & T033320 & C13T033320 \\
\hline IJ 64 Y EP & Epson & Yellow & T034420 & C13T034420 \\
IJ 65 Y BR & Brother & Yellow & LC25Y & LC25Y \\
\hline IJ 66 C BR & Brother & Cyan & LC25C & LC25C \\
IJ 67 M BR & Brother & Magenta & LC25M & LC25M \\
\hline IJ 68 K BR & Brother & Black & LC71BK & LC71BK \\
IJ 69 K SH & Sharp & Black & AJ-C50B & AJ-C50B \\
\hline IJ 70 C SH & Sharp & Cyan Tri-Color & AJ-C50C & AJ-C50C \\
IJ 71 Y SH & Sharp & Yellow Tri-Color & AJ-C50C & AJ-C50C \\
IJ 72 M SH & Sharp & Magenta Tri-Color & AJ-C50C & AJ-C50C \\
IJ 73 K DL & Dell & Black & 9 & MK990 \\
IJ 74 K DL & Dell & Black & 21 & W498D \\
IJ 75 C DL & Dell & Cyan Tri-Color & 5 & J5567 \\
IJ 76 Y DL & Dell & Yellow Tri-Color & 5 & J5567 \\
IJ 77 M DL & Dell & Magenta Tri-Color & 5 & J5567 \\
\hline IJ 78 LC HP & Hewlett & Light Cyan & 81 & C4934A \\
\hline
\end{tabular}

Toner Collection

\begin{tabular}{|c|c|c|c|c|}
\hline Sample ID & Brand & Color & Model & Manufacturer \# \\
\hline TN 01 K BR & Brother & Black & TN-360 & TN-360 \\
\hline TN 02 K HPCN & $\begin{array}{l}\text { HB (HP/Canon) } \\
\text { "Hummingbird" }\end{array}$ & Black & N/A & N/A \\
\hline TN 03 C HPCN & $\begin{array}{l}\text { HB (HP/Canon) } \\
\text { "Hummingbird" }\end{array}$ & Cyan & N/A & N/A \\
\hline TN 04 M HPCN & $\begin{array}{l}\text { HB (HP/Canon) } \\
\text { "Hummingbird" }\end{array}$ & Magenta & $\mathrm{N} / \mathrm{A}$ & N/A \\
\hline TN 05 Y HPCN & $\begin{array}{l}\text { HB (HP/Canon) } \\
\text { "Hummingbird" }\end{array}$ & Yellow & N/A & N/A \\
\hline TN 06 K HP & $\begin{array}{l}\text { Hewlett } \\
\text { Packard }\end{array}$ & Black & $124 \mathrm{~A}$ & Q6000A \\
\hline TN 07 K HP & $\begin{array}{l}\text { Hewlett } \\
\text { Packard }\end{array}$ & Black & $124 \mathrm{~A}$ & Q6000A \\
\hline TN 08 K HP & $\begin{array}{l}\text { Hewlett } \\
\text { Packard }\end{array}$ & Black & $124 \mathrm{~A}$ & Q6000A \\
\hline
\end{tabular}




\begin{tabular}{|c|c|c|c|c|}
\hline Sample ID & Brand & Color & Model & Manufacturer \# \\
\hline TN 09 K HP & $\begin{array}{l}\text { Hewlett } \\
\text { Packard }\end{array}$ & Black & $05 \mathrm{~A}$ & CE505A \\
\hline TN 10 K HP & $\begin{array}{l}\text { Hewlett } \\
\text { Packard }\end{array}$ & Black & $125 \mathrm{~A}$ & CB540A \\
\hline TN 11 K HP & $\begin{array}{l}\text { Hewlett } \\
\text { Packard }\end{array}$ & Black & $304 \mathrm{~A}$ & CC530A \\
\hline TN 12 K HP & $\begin{array}{l}\text { Hewlett } \\
\text { Packard }\end{array}$ & Black & $05 \mathrm{X}$ & CE505X \\
\hline TN 13 K HP & $\begin{array}{l}\text { Hewlett } \\
\text { Packard }\end{array}$ & Black & $05 \mathrm{~A}$ & CE505A \\
\hline TN 14 K HP & $\begin{array}{l}\text { Hewlett } \\
\text { Packard }\end{array}$ & Black & $11 \mathrm{~A}$ & Q6511A \\
\hline TN 15 K HP & $\begin{array}{l}\text { Hewlett } \\
\text { Packard }\end{array}$ & Black & $55 \mathrm{~A}$ & CE255A \\
\hline TN 16 K HP & $\begin{array}{l}\text { Hewlett } \\
\text { Packard }\end{array}$ & Black & $55 \mathrm{~A}$ & CE255A \\
\hline TN 17 K HP & $\begin{array}{l}\text { Hewlett } \\
\text { Packard }\end{array}$ & Black & $55 \mathrm{~A}$ & CE255A \\
\hline TN 18 K HP & $\begin{array}{l}\text { Hewlett } \\
\text { Packard }\end{array}$ & Black & $11 \mathrm{~A}$ & Q6511A \\
\hline TN 19 K HP & $\begin{array}{l}\text { Hewlett } \\
\text { Packard }\end{array}$ & Black & $05 \mathrm{X}$ & CE505X \\
\hline TN $20 \mathrm{~K}$ HP & $\begin{array}{l}\text { Hewlett } \\
\text { Packard }\end{array}$ & Black & $12 \mathrm{~A}$ & Q2612A \\
\hline TN $21 \mathrm{~K}$ HP & $\begin{array}{l}\text { Hewlett } \\
\text { Packard }\end{array}$ & Black & $305 \mathrm{~A}$ & CE410A \\
\hline TN 22 C HP & $\begin{array}{l}\text { Hewlett } \\
\text { Packard }\end{array}$ & Cyan & $125 \mathrm{~A}$ & CB541A \\
\hline TN 23 C HP & $\begin{array}{l}\text { Hewlett } \\
\text { Packard }\end{array}$ & Cyan & $305 \mathrm{~A}$ & CE411A \\
\hline TN 24 M HP & $\begin{array}{l}\text { Hewlett } \\
\text { Packard }\end{array}$ & Magenta & $125 \mathrm{~A}$ & CB543A \\
\hline TN 25 M HP & $\begin{array}{l}\text { Hewlett } \\
\text { Packard }\end{array}$ & Magenta & $305 \mathrm{~A}$ & CE413A \\
\hline TN 26 Y HP & $\begin{array}{l}\text { Hewlett } \\
\text { Packard }\end{array}$ & Yellow & $125 \mathrm{~A}$ & CB542A \\
\hline TN 27 Y HP & $\begin{array}{l}\text { Hewlett } \\
\text { Packard }\end{array}$ & Yellow & $305 \mathrm{~A}$ & CE412A \\
\hline TN 28 K IMX & IMEX 2300 & Black & $\mathrm{N} / \mathrm{A}$ & N/A \\
\hline TN 29 C IMX & IMEX 2300 & Cyan & $\mathrm{N} / \mathrm{A}$ & N/A \\
\hline TN 30 M IMX & IMEX 2300 & Magenta & $\mathrm{N} / \mathrm{A}$ & $\mathrm{N} / \mathrm{A}$ \\
\hline TN 31 Y IMX & IMEX 2300 & Yellow & $\mathrm{N} / \mathrm{A}$ & $\mathrm{N} / \mathrm{A}$ \\
\hline TN 32 K KN & $\begin{array}{c}\text { KM2300 } \\
\text { Konica }\end{array}$ & Black & N/A & N/A \\
\hline
\end{tabular}




\begin{tabular}{|c|c|c|c|c|}
\hline Sample ID & Brand & Color & Model & Manufacturer \# \\
\hline TN 33 C KN & $\begin{array}{l}\text { KM2300 } \\
\text { Konica }\end{array}$ & Cyan & $\mathrm{N} / \mathrm{A}$ & N/A \\
\hline TN 34 M KN & $\begin{array}{l}\text { KM2300 } \\
\text { Konica }\end{array}$ & Magenta & $\mathrm{N} / \mathrm{A}$ & N/A \\
\hline TN 35 Y KN & $\begin{array}{l}\text { KM2300 } \\
\text { Konica }\end{array}$ & Yellow & N/A & N/A \\
\hline TN 36 K OD & Office Depot & Black & $\mathrm{N} / \mathrm{A}$ & OD53JP \\
\hline TN 37 K OKI & OKI & Black & 43381904 & 43381904 \\
\hline TN 38 K OKI & OKI & Black & 43381904 & 43381904 \\
\hline TN 39 C OKI & OKI & Cyan & 43381904 & 43381904 \\
\hline TN 40 M OKI & OKI & Magenta & 43381904 & 43381904 \\
\hline TN 41 Y OKI & OKI & Yellow & 43381904 & 43381904 \\
\hline TN 42 K XR & X6200 Xerox & Black & N/A & N/A \\
\hline TN 43 C XR & X6200 Xerox & Cyan & N/A & N/A \\
\hline TN 44 M XR & X6200 Xerox & Magenta & $\mathrm{N} / \mathrm{A}$ & N/A \\
\hline TN 45 Y XR & X6200 Xerox & Yellow & $\mathrm{N} / \mathrm{A}$ & N/A \\
\hline TN 46 K XK & XeiKon & Black & N/A & N/A \\
\hline TN 47 C XK & XeiKon & Cyan & N/A & N/A \\
\hline TN 48 M XK & XeiKon & Magenta & $\mathrm{N} / \mathrm{A}$ & N/A \\
\hline TN 49 Y XK & XeiKon & Yellow & N/A & $\mathrm{N} / \mathrm{A}$ \\
\hline TN 50 K BR & Brother & Black & $\mathrm{TN}-420$ & $\mathrm{TN}-420$ \\
\hline TN $51 \mathrm{~K}$ XR & Xerox & Black & $857 X$ & \\
\hline TN 52 K RC & $\mathrm{RICOH}$ & Black & $1357 \mathrm{EX}$ & 828080(EDP) \\
\hline TN 53 K RC & $\mathrm{RICOH}$ & Black & 1357EX & $828080(\mathrm{EDP})$ \\
\hline TN 54 K HP & $\begin{array}{l}\text { Hewlett } \\
\text { Packard }\end{array}$ & Black & $305 \mathrm{~A}$ & CE410A \\
\hline TN 55 Y HP & $\begin{array}{l}\text { Hewlett } \\
\text { Packard }\end{array}$ & Yellow & $304 \mathrm{~A}$ & CC532A \\
\hline TN 56 K HP & $\begin{array}{l}\text { Hewlett } \\
\text { Packard }\end{array}$ & Black & $304 \mathrm{~A}$ & CC530A \\
\hline TN 57 K TS & Toshiba & Black & $\begin{array}{c}\text { T-7200 } \\
\text { estudio853 }\end{array}$ & 9940-A \\
\hline TN $58 \mathrm{~K} \mathrm{CN}$ & Canon & Black & LC120/720 & $\begin{array}{c}2 \mathrm{G} 21 \mathrm{H} 1 \mathrm{Ga}, \\
20120721\end{array}$ \\
\hline TN $60 \mathrm{~K} \mathrm{CN}$ & Canon & Black & $\begin{array}{l}\text { GPR-32 } \\
\text { printer }\end{array}$ & \\
\hline TN 61 Y OKI & OKI & Yellow & 430668 & $\begin{array}{c}2 \mathrm{~K} 00 \mathrm{C} 8205 \mathrm{CY} 31 \\
1396\end{array}$ \\
\hline TN $62 \mathrm{~K} \mathrm{CN}$ & Canon & Black & CLC1100 & CLC1100 \\
\hline TN 63 C CN & Canon & Cyan & 116 & 116 \\
\hline TN 64 M CN & Canon & Magenta & 116 & 116 \\
\hline TN 65 Y CN & Canon & Yellow & 116 & 116 \\
\hline
\end{tabular}




\begin{tabular}{ccccc}
\hline Sample ID & Brand & Color & Model & Manufacturer \# \\
\hline TN 66 K DL & Dell & Black & J9833 & J9833 \\
TN 67 C DL & Dell & Cyan & C5GC3 & C5GC3 \\
TN 68 M DL & Dell & Magenta & XMX5D & XMX5D \\
TN 69 K PN & Panasonic & Black & KX-FA83 & KX-FA83 \\
TN 70 C RC & RICOH & Cyan & GC 21C & J735-17 \\
TN 71 M RC & RICOH & Magenta & GC 21M & J736-17 \\
TN 72 Y RC & RICOH & Yellow & GC 21Y & J737-17 \\
TN 73 K SM & Samsung & Black & K409 & CLT-K409S \\
TN 74 C SM & Samsung & Cyan & C407 & CLT-C407S \\
TN 75 M SM & Samsung & Magenta & M407 & CLT-M407S \\
TN 76 Y SM & Samsung & Yellow & Y409 & CLT-Y409S \\
TN 77 K SH & Sharp & Black & AL-110TD & AL-110TD \\
\hline
\end{tabular}

Offset Collection

\begin{tabular}{cccc}
\hline Sample ID & Manufacturer & Color & Sample Type \\
\hline OF 01 C FG & Flint Group & Cyan & Printout \\
OF 02 M FG & Flint Group & Magenta & Printout \\
OF 03 Y FG & Flint Group & Yellow & Printout \\
OF 04 K FG & Flint Group & Black & Printout \\
OF 05 B ECK & Eckart & Brown & Printout \\
OF 06 LY FG & Flint Group & Light Yellow & Printout \\
OF 07 DC FG & Flint Group & Dark Cyan & Printout \\
OF 08 C DG & DayGlo & Cyan & Printout \\
OF 09 K TI & Toyo Ink & Black & Printout \\
OF 10 C TI & Toyo Ink & Cyan & Printout \\
OF 11 M TI & Toyo Ink & Magenta & Printout \\
OF 12 Y TI & Toyo Ink & Yellow & Printout \\
OF 13 K SC & Sun Chemical & Black & Printout \\
OF 14 C SC & Sun Chemical & Cyan & Printout \\
OF 15 Y SC & Sun Chemical & Yellow & Printout \\
OF 16 M SC & Sun Chemical & Magenta & Printout \\
OF 17 K SC & Sun Chemical & Black & Printout \\
OF 18 C SC & Sun Chemical & Cyan & Printout \\
OF 19 M SC & Sun Chemical & Magenta & Printout \\
OF 20 Y SC & Sun Chemical & Yellow & Printout \\
OF 21 K GI & Graphic Ink Co. & Black & Printout \\
OF 22 Y GI & Graphic Ink Co. & Yellow & Printout \\
OF 23 M GI & Graphic Ink Co. & Magenta & Printout \\
\hline
\end{tabular}




\begin{tabular}{|c|c|c|c|}
\hline Sample ID & Manufacturer & Color & Sample Type \\
\hline OF 24 DC GI & Graphic Ink Co. & Dark Cyan & Printout \\
\hline OF 25 C GI & Graphic Ink Co. & Cyan & Printout \\
\hline OF 26 LY GI & Graphic Ink Co. & Light Yellow & Printout \\
\hline OF 27 Y GI & Graphic Ink Co. & Yellow & Printout \\
\hline OF 28 DO GI & Graphic Ink Co. & Dark Orange & Printout \\
\hline OF 29 DO GI & Graphic Ink Co. & Dark Orange & Printout \\
\hline OF 30 O GI & Graphic Ink Co. & Orange & Printout \\
\hline OF 31 R GI & Graphic Ink Co. & Red & Printout \\
\hline OF 32 R GI & Graphic Ink Co. & Red & Printout \\
\hline OF 33 DP GI & Graphic Ink Co. & Dark Pink & Printout \\
\hline OF 34 DP GI & Graphic Ink Co. & Dark Pink & Printout \\
\hline OF 35 PR GI & Graphic Ink Co. & Purple & Printout \\
\hline OF 36 DPR GI & Graphic Ink Co. & Dark Purple & Printout \\
\hline OF 37 DPR GI & Graphic Ink Co. & Dark Purple & Printout \\
\hline OF 38 DC GI & Graphic Ink Co. & Dark Cyan & Printout \\
\hline OF 39 G GI & Graphic Ink Co. & Green & Printout \\
\hline OF 40 RIT & N/A & Black & Business Card \\
\hline OF 41 LIVI & N/A & Green & Visa \\
\hline OF 42 LIVI & N/A & Pink/Peach & Visa \\
\hline OF 43 LIVI & N/A & $\begin{array}{l}\text { Blue \& Orange } \\
\text { Stripes }\end{array}$ & Visa \\
\hline OF 44 LIVI & N/A & Red & Visa \\
\hline OF 45 LIVI & $\mathrm{N} / \mathrm{A}$ & Blue & Visa \\
\hline OF 46 LIVI & $\mathrm{N} / \mathrm{A}$ & Bronze & Visa \\
\hline OF 47 LIVI & $\mathrm{N} / \mathrm{A}$ & Green & Visa \\
\hline OF 48 LIVI & N/A & Brown/Orange & Visa \\
\hline OF 49 USPB & N/A & $\begin{array}{l}\text { Blue/Pink/Orange } \\
\text { Lines }\end{array}$ & US Passport \\
\hline OF 50 USPB & N/A & Orange Stripes & US Passport \\
\hline OF 51 USPB & N/A & Red & US Passport \\
\hline OF 52 USPB & N/A & $\begin{array}{l}\text { Blue (solid blue side } \\
\text { with white circles) }\end{array}$ & US Passport \\
\hline OF 53 USPB & N/A & Red & US Passport \\
\hline OF 54 USPB & N/A & Red & US Passport \\
\hline OF 55 USPB & N/A & Blue & US Passport \\
\hline OF 56 USPN & N/A & Yellow & US Passport \\
\hline OF 57 USPN & N/A & Black & US Passport \\
\hline OF 58 USPN & N/A & Light Blue & US Passport \\
\hline OF 59 USPN & N/A & Black & US Passport \\
\hline OF 60 USPN & N/A & Red & US Passport \\
\hline OF 61 USPN & N/A & Blue & US Passport \\
\hline
\end{tabular}




\begin{tabular}{cccc}
\hline Sample ID & Manufacturer & Color & Sample Type \\
\hline OF 62 USPN & N/A & Black & US Passport \\
OF 63 USPN & N/A & Dark Blue & US Passport \\
\hline OF 64 K UB & $\begin{array}{c}\text { Unibrilliant } \\
\text { (Germany) }\end{array}$ & Black & Paste \\
\hline OF 65 C UB & $\begin{array}{c}\text { Unibrilliant } \\
\text { (Germany) }\end{array}$ & Cyan & Paste \\
OF 66 M UB & $\begin{array}{l}\text { Unibrilliant } \\
\text { (Germany) }\end{array}$ & Magenta & Paste \\
OF 67 Y UB & Unibrilliant & Yellow & Paste \\
OF 68 K SC & Sun Chemical & Black & Paste \\
OF 69 C SC & Sun Chemical & Cyan & Paste \\
OF 70 M SC & Sun Chemical & Magenta & Paste \\
OF 71 Y SC & Sun Chemical & Yellow & Paste \\
OF 72 K SC & Sun Chemical & Black & Paste \\
OF 73 C SC & Sun Chemical & Cyan & Paste \\
OF 74 M SC & Sun Chemical & Magenta & Paste \\
OF 75 Y SC & Sun Chemical & Yellow & Paste \\
OF 76 K SC & Sun Chemical & Black & Paste \\
OF 77 C SC & Sun Chemical & Cyan & Paste \\
OF 78 M SC & Sun Chemical & Magenta & Paste \\
OF 79 Y SC & Sun Chemical & Yellow & Paste \\
\hline
\end{tabular}

Intaglio Collection

\begin{tabular}{|c|c|c|c|c|c|}
\hline Sample ID & Country & Denomination & Color & $\begin{array}{l}\text { Series } \\
\text { Year }\end{array}$ & Remarks \\
\hline IT 01 DG UZB & Uzbekistan & 1000 & $\begin{array}{l}\text { Dark } \\
\text { Green }\end{array}$ & 2001 & \\
\hline IT 02 BR NEP & Nepal & 5 & Brown & 2009 & $\begin{array}{c}\text { IT } 02 \& 81 \\
\text { same banknote }\end{array}$ \\
\hline IT 03 DB HAI & Haiti & 100 & $\begin{array}{l}\text { Dark } \\
\text { Blue }\end{array}$ & 2004 & $\begin{array}{c}\text { IT } 03 \& 82 \\
\text { same banknote }\end{array}$ \\
\hline IT 04 DG ARG & Argentina & 5 & $\begin{array}{l}\text { Dark } \\
\text { Green }\end{array}$ & 1997 & \\
\hline IT 05 AQ BAR & Barbados & 2 & Aqua & 2007 & $\begin{array}{l}\text { IT } 05-08 \text { same } \\
\text { banknote }\end{array}$ \\
\hline IT 06 DM BAR & Barbados & 2 & $\begin{array}{l}\text { Dark } \\
\text { Magenta }\end{array}$ & 2007 & $\begin{array}{l}\text { IT } 05-08 \text { same } \\
\text { banknote }\end{array}$ \\
\hline IT 07 DB BAR & Barbados & 2 & Dark & 2007 & IT 05-08 same \\
\hline
\end{tabular}




\begin{tabular}{|c|c|c|c|c|c|}
\hline Sample ID & Country & Denomination & Color & $\begin{array}{c}\text { Series } \\
\text { Year } \\
\end{array}$ & Remarks \\
\hline & & & Blue & & banknote \\
\hline IT 08 DB BAR & Barbados & 2 & $\begin{array}{l}\text { Dark } \\
\text { Blue }\end{array}$ & 2007 & $\begin{array}{c}\text { IT 05-08 same } \\
\text { banknote }\end{array}$ \\
\hline IT 09 K UKR & Ukraine & 10 & Black & 2011 & $\begin{array}{l}\text { IT 09-11 same } \\
\text { banknote }\end{array}$ \\
\hline IT 10 R UKR & Ukraine & 10 & Red & 2011 & $\begin{array}{c}\text { IT 09-11 same } \\
\text { banknote }\end{array}$ \\
\hline IT 11 GL UKR & Ukraine & 10 & Gold & 2011 & $\begin{array}{l}\text { IT 09-11 same } \\
\text { banknote }\end{array}$ \\
\hline IT 12 K RUS & Russia & 50 & Black & 1997 & $\begin{array}{l}\text { IT } 12,13, \& 83 \\
\text { same banknote }\end{array}$ \\
\hline IT 13 K RUS & Russia & 50 & Black & 1997 & $\begin{array}{l}\text { IT } 12,13, \& 83 \\
\text { same banknote }\end{array}$ \\
\hline IT 14 BR RUS & Russia & 100 & Brown & 1997 & $\begin{array}{c}\text { IT } 14,15, \& 84 \\
\text { same banknote }\end{array}$ \\
\hline IT 15 BR RUS & Russia & 100 & Brown & 1997 & $\begin{array}{l}\text { IT } 14,15, \& 84 \\
\text { same banknote }\end{array}$ \\
\hline IT 16 DB CAN & Canada & 5 & $\begin{array}{l}\text { Dark } \\
\text { Blue }\end{array}$ & 2006 & $\begin{array}{c}\text { IT } 16 \& 17 \\
\text { same banknote }\end{array}$ \\
\hline IT 17 DB CAN & Canada & 5 & $\begin{array}{l}\text { Dark } \\
\text { Blue }\end{array}$ & 2006 & $\begin{array}{c}\text { IT } 16 \& 17 \\
\text { same banknote }\end{array}$ \\
\hline IT 18 B KOR & $\begin{array}{l}\text { Korea } \\
\text { (South) }\end{array}$ & 1000 & Blue & 2007 & $\begin{array}{l}\text { IT } 18-20 \& 85 \\
\text { same banknote }\end{array}$ \\
\hline IT 19 PR KOR & $\begin{array}{l}\text { Korea } \\
\text { (South) }\end{array}$ & 1000 & Purple & 2007 & $\begin{array}{l}\text { IT } 18-20 \& 85 \\
\text { same banknote }\end{array}$ \\
\hline IT 20 PR KOR & $\begin{array}{l}\text { Korea } \\
\text { (South) }\end{array}$ & 1000 & Purple & 2007 & $\begin{array}{l}\text { IT 18-20 \& } 85 \\
\text { same banknote }\end{array}$ \\
\hline IT 21 DB GUA & Guatemala & 5 & $\begin{array}{l}\text { Dark } \\
\text { Blue }\end{array}$ & 2008 & $\begin{array}{l}\text { IT } 21-23 \text { same } \\
\text { banknote }\end{array}$ \\
\hline IT 22 DB GUA & Guatemala & 5 & $\begin{array}{l}\text { Dark } \\
\text { Blue }\end{array}$ & 2008 & $\begin{array}{c}\text { IT } 21-23 \text { same } \\
\text { banknote }\end{array}$ \\
\hline IT 23 DB GUA & Guatemala & 5 & $\begin{array}{l}\text { Dark } \\
\text { Blue }\end{array}$ & 2008 & $\begin{array}{l}\text { IT } 21-23 \text { same } \\
\text { banknote }\end{array}$ \\
\hline IT 24 B UKR & Ukraine & 5 & Blue & 2005 & \\
\hline IT 25 BR UKR & Ukraine & 2 & Brown & 2011 & \\
\hline IT 26 G CHI & China & 1 & Green & 1999 & \\
\hline IT 27 R HON & Honduras & 1 & Red & 2006 & \\
\hline IT 28 R PER & Peru & 10 & Red & 2009 & $\begin{array}{l}\text { IT } 28-31 \& 85 \\
\text { same banknote }\end{array}$ \\
\hline IT 29 G PER & Peru & 10 & Green & 2009 & $\begin{array}{l}\text { IT } 28-31 \& 85 \\
\text { same banknote }\end{array}$ \\
\hline IT 30 G PER & Peru & 10 & Green & 2009 & $\begin{array}{l}\text { IT } 28-31 \& 85 \\
\text { same banknote }\end{array}$ \\
\hline
\end{tabular}




\begin{tabular}{|c|c|c|c|c|c|}
\hline Sample ID & Country & Denomination & Color & $\begin{array}{c}\text { Series } \\
\text { Year }\end{array}$ & Remarks \\
\hline IT 31 O PER & Peru & 10 & Orange & 2009 & $\begin{array}{l}\text { IT } 28-31 \& 85 \\
\text { same banknote }\end{array}$ \\
\hline IT 32 R TRI & $\begin{array}{c}\text { Trinidad \& } \\
\text { Tobago }\end{array}$ & 1 & Red & 2006 & $\begin{array}{l}\text { IT } 32 \& 33 \\
\text { same banknote }\end{array}$ \\
\hline IT 33 MA TRI & $\begin{array}{c}\text { Trinidad \& } \\
\text { Tobago }\end{array}$ & 1 & Maroon & 2006 & $\begin{array}{c}\text { IT } 32 \& 33 \\
\text { same banknote }\end{array}$ \\
\hline IT 34 G EUR & $\begin{array}{l}\text { Europe } \\
\text { (Italy) }\end{array}$ & 5 & Green & 2002 & $\begin{array}{c}\text { IT 34-36 same } \\
\text { banknote }\end{array}$ \\
\hline IT 35 BR EUR & $\begin{array}{l}\text { Europe } \\
\text { (Italy) }\end{array}$ & 5 & Brown & 2002 & $\begin{array}{l}\text { IT 34-36 same } \\
\text { banknote }\end{array}$ \\
\hline IT 36 BR EUR & $\begin{array}{l}\text { Europe } \\
\text { (Italy) }\end{array}$ & 5 & Brown & 2002 & $\begin{array}{l}\text { IT 34-36 same } \\
\text { banknote }\end{array}$ \\
\hline IT 37 R CUB & Cuba & 3 & Red & 2006 & $\begin{array}{c}\text { IT } 37 \& 38 \\
\text { same banknote }\end{array}$ \\
\hline IT 38 G CUB & Cuba & 3 & Green & 2006 & $\begin{array}{c}\text { IT } 37 \& 38 \\
\text { same banknote }\end{array}$ \\
\hline IT 39 DG CTR & Costa Rica & 10000 & $\begin{array}{l}\text { Dark } \\
\text { Green }\end{array}$ & 2009 & $\begin{array}{c}\text { IT 39-42 same } \\
\text { banknote }\end{array}$ \\
\hline IT 40 G CTR & Costa Rica & 10000 & Green & 2009 & $\begin{array}{l}\text { IT 39-42 same } \\
\text { banknote }\end{array}$ \\
\hline IT 41 G CTR & Costa Rica & 10000 & Green & 2009 & $\begin{array}{l}\text { IT 39-42 same } \\
\text { banknote }\end{array}$ \\
\hline IT 42 G CTR & Costa Rica & 10000 & Green & 2009 & $\begin{array}{c}\text { IT 39-42 same } \\
\text { banknote }\end{array}$ \\
\hline IT 43 DY CTR & Costa Rica & 5000 & $\begin{array}{c}\text { Dark } \\
\text { Yellow }\end{array}$ & 2009 & $\begin{array}{c}\text { IT } 43-46 \text { same } \\
\text { banknote }\end{array}$ \\
\hline IT 44 MA CTR & Costa Rica & 5000 & Maroon & 2009 & $\begin{array}{c}\text { IT } 43-46 \text { same } \\
\text { banknote }\end{array}$ \\
\hline IT 45 Y CTR & Costa Rica & 5000 & Yellow & 2009 & $\begin{array}{c}\text { IT } 43-46 \text { same } \\
\text { banknote }\end{array}$ \\
\hline IT 46 DY CTR & Costa Rica & 5000 & $\begin{array}{c}\text { Dark } \\
\text { Yellow }\end{array}$ & 2009 & $\begin{array}{c}\text { IT } 43-46 \text { same } \\
\text { banknote }\end{array}$ \\
\hline IT 47 G CAY & $\begin{array}{c}\text { Cayman } \\
\text { Islands }\end{array}$ & 5 & Green & 1996 & $\begin{array}{c}\text { IT } 47 \& 86 \\
\text { same banknote }\end{array}$ \\
\hline IT 48 BR UAE & $\begin{array}{c}\text { United Arab } \\
\text { Emirates }\end{array}$ & 5 & Brown & 2009 & \\
\hline IT 49 AQ TUN & Tunisia & 10 & Aqua & 1994 & $\begin{array}{c}\text { IT 49-51 same } \\
\text { banknote }\end{array}$ \\
\hline IT 50 DB TUN & Tunisia & 10 & $\begin{array}{l}\text { Dark } \\
\text { Blue }\end{array}$ & 1994 & $\begin{array}{c}\text { IT 49-51 same } \\
\text { banknote }\end{array}$ \\
\hline IT 51 DB TUN & Tunisia & 10 & $\begin{array}{l}\text { Dark } \\
\text { Blue }\end{array}$ & 1994 & $\begin{array}{c}\text { IT 49-51 same } \\
\text { banknote }\end{array}$ \\
\hline IT $52 \mathrm{~K}$ TRI & Trinidad \& & 10 & Black & Unknown & \\
\hline
\end{tabular}




\begin{tabular}{|c|c|c|c|c|c|}
\hline Sample ID & Country & Denomination & Color & $\begin{array}{c}\text { Series } \\
\text { Year } \\
\end{array}$ & Remarks \\
\hline \multicolumn{6}{|c|}{ Tobago } \\
\hline IT 53 BR NZE & $\begin{array}{c}\text { New } \\
\text { Zealand }\end{array}$ & 5 & $\begin{array}{l}\text { Brownis } \\
\text { h-orange }\end{array}$ & Unknown & $\begin{array}{l}\text { Polymer } \\
\text { banknote }\end{array}$ \\
\hline IT 54 K RIT & $\mathrm{N} / \mathrm{A}$ & N/A & Black & N/A & Business card \\
\hline IT 55 G RIT & N/A & N/A & Green & N/A & Business card \\
\hline IT 56 B LIVI & N/A & N/A & Blue & N/A & Visa \\
\hline IT 57 R LIVI & N/A & N/A & Red & N/A & Visa \\
\hline IT 58 GL LIVI & $\mathrm{N} / \mathrm{A}$ & N/A & Gold & N/A & Visa \\
\hline IT 59 G CZE & $\begin{array}{l}\text { Czech } \\
\text { Republic }\end{array}$ & 100 & Green & 1997 & \\
\hline IT $60 \mathrm{~K} \mathrm{JAM}$ & Jamaica & 500 & Black & 2012 & $\begin{array}{c}\text { IT } 60-62 \text { same } \\
\text { banknote }\end{array}$ \\
\hline IT 61 MA ЈАM & Jamaica & 500 & Maroon & 2012 & $\begin{array}{c}\text { IT } 60-62 \text { same } \\
\text { banknote }\end{array}$ \\
\hline IT 62 BR JAM & Jamaica & 500 & Brown & 2012 & $\begin{array}{c}\text { IT } 60-62 \text { same } \\
\text { banknote }\end{array}$ \\
\hline IT 63 B NZE & $\begin{array}{l}\text { New } \\
\text { Zealand }\end{array}$ & 10 & Blue & Unknown & $\begin{array}{c}\text { Polymer } \\
\text { banknote; IT } 63 \\
\& 64 \text { same } \\
\text { banknote }\end{array}$ \\
\hline IT 64 B NZE & $\begin{array}{l}\text { New } \\
\text { Zealand }\end{array}$ & 10 & Blue & Unknown & $\begin{array}{c}\text { Polymer } \\
\text { banknote; IT } 63 \\
\text { \& } 64 \text { same } \\
\text { banknote }\end{array}$ \\
\hline IT 65 G NZE & $\begin{array}{c}\text { New } \\
\text { Zealand }\end{array}$ & 20 & Green & Unknown & $\begin{array}{l}\text { Polymer } \\
\text { banknote }\end{array}$ \\
\hline IT 66 B CTR & Costa Rica & 2000 & Blue & 2009 & $\begin{array}{c}\text { IT } 66-69 \text { same } \\
\text { banknote }\end{array}$ \\
\hline IT 67 B CTR & Costa Rica & 2000 & Blue & 2009 & $\begin{array}{c}\text { IT } 66-69 \text { same } \\
\text { banknote }\end{array}$ \\
\hline IT 68 LB CTR & Costa Rica & 2000 & $\begin{array}{l}\text { Light } \\
\text { Blue }\end{array}$ & 2009 & $\begin{array}{c}\text { IT 66-69 same } \\
\text { banknote }\end{array}$ \\
\hline IT 69 B CTR & Costa Rica & 2000 & Blue & 2009 & $\begin{array}{c}\text { IT } 66-69 \text { same } \\
\text { banknote }\end{array}$ \\
\hline IT 70 PR CHI & China & 5 & Purple & 2005 & \\
\hline IT $71 \mathrm{~K} \mathrm{CHI}$ & China & 10 & Black & 2005 & \\
\hline IT 72 G EUR & $\begin{array}{l}\text { Europe } \\
\text { (Italy) }\end{array}$ & 5 & Green & 2013 & $\begin{array}{c}\text { IT 72-74 same } \\
\text { banknote }\end{array}$ \\
\hline IT 73 G EUR & $\begin{array}{l}\text { Europe } \\
\text { (Italy) }\end{array}$ & 5 & Green & 2013 & $\begin{array}{c}\text { IT } 72-74 \text { same } \\
\text { banknote }\end{array}$ \\
\hline IT 74 G EUR & $\begin{array}{l}\text { Europe } \\
\text { (Italy) }\end{array}$ & 5 & Green & 2013 & $\begin{array}{c}\text { IT 72-74 same } \\
\text { banknote }\end{array}$ \\
\hline
\end{tabular}




\begin{tabular}{|c|c|c|c|c|c|}
\hline Sample ID & Country & Denomination & Color & $\begin{array}{c}\text { Series } \\
\text { Year }\end{array}$ & Remarks \\
\hline IT 75 K USA & $\begin{array}{l}\text { United } \\
\text { States }\end{array}$ & 1 & Black & 2006 & $\begin{array}{c}\text { IT } 75 \& 76 \\
\text { same banknote }\end{array}$ \\
\hline IT 76 G USA & $\begin{array}{l}\text { United } \\
\text { States }\end{array}$ & 1 & Green & 2006 & $\begin{array}{c}\text { IT } 75 \& 76 \\
\text { same banknote }\end{array}$ \\
\hline IT 77 K USA & $\begin{array}{l}\text { United } \\
\text { States }\end{array}$ & 5 & Black & 2006 & $\begin{array}{c}\text { IT } 77 \& 78 \\
\text { same banknote }\end{array}$ \\
\hline IT 78 G USA & $\begin{array}{l}\text { United } \\
\text { States }\end{array}$ & 5 & Green & 2006 & $\begin{array}{c}\text { IT } 77 \& 78 \\
\text { same banknote }\end{array}$ \\
\hline IT 79 K USA & $\begin{array}{l}\text { United } \\
\text { States }\end{array}$ & Unknown & Black & Unknown & $\begin{array}{l}\text { Shredded US } \\
\text { currency }\end{array}$ \\
\hline IT 80 G USA & $\begin{array}{l}\text { United } \\
\text { States }\end{array}$ & Unknown & Green & Unknown & $\begin{array}{l}\text { Shredded US } \\
\text { currency }\end{array}$ \\
\hline IT 81 PR NEP & Nepal & 5 & Purple & 2009 & $\begin{array}{c}\text { IT } 02 \& 81 \\
\text { same banknote }\end{array}$ \\
\hline IT 82 DB HAI & Haiti & 100 & $\begin{array}{l}\text { Dark } \\
\text { Blue }\end{array}$ & 2004 & $\begin{array}{c}\text { IT } 03 \& 82 \\
\text { same banknote }\end{array}$ \\
\hline IT 83 B RUS & Russia & 50 & Blue & 1997 & $\begin{array}{l}\text { IT } 12,13, \& 83 \\
\text { same banknote }\end{array}$ \\
\hline IT 84 BR RUS & Russia & 100 & Brown & 1997 & $\begin{array}{l}\text { IT } 14,15, \& 84 \\
\text { same banknote }\end{array}$ \\
\hline IT 85 B KOR & $\begin{array}{l}\text { Korea } \\
\text { (South) }\end{array}$ & 1000 & Blue & 2007 & $\begin{array}{l}\text { IT } 18-20 \& 85 \\
\text { same banknote }\end{array}$ \\
\hline IT 86 R CAY & $\begin{array}{l}\text { Cayman } \\
\text { Islands }\end{array}$ & 5 & Red & 1996 & $\begin{array}{c}\text { IT } 47 \& 86 \\
\text { same banknote }\end{array}$ \\
\hline
\end{tabular}


Appendix B

FIU Vehicle Glass

\begin{tabular}{cccc}
\hline $\begin{array}{c}\text { Sample } \\
\text { ID }\end{array}$ & VIN & Vehicle Make \& Model & Year \\
\hline $\mathbf{0 0 1}$ & 4T1BE32K94U272646 & Toyota Camry & 2004 \\
$\mathbf{0 0 2}$ & 2HGFG3B54DH500424 & Honda Civic & 2013 \\
$\mathbf{0 0 3}$ & 4JGCB65E18A071448 & Mercedes R350 & 2008 \\
$\mathbf{0 0 4}$ & 2HGFG12629H527108 & Honda Civic & 2009 \\
$\mathbf{0 0 5}$ & 1FA6P8CF7G5224485 & Ford Mustang & 2016 \\
$\mathbf{0 0 6}$ & 1YVHP80C185M32614 & Mazda 6 & 2008 \\
$\mathbf{0 0 7}$ & WMWXP5C59G3B76438 & Mini Cooper & 2016 \\
$\mathbf{0 0 8}$ & JTDKTUD35CD500860 & Toyota Yaris & 2012 \\
$\mathbf{0 0 9}$ & JTDZN3EU1C3024669 & Toyota Prius v & 2012 \\
$\mathbf{0 1 0}$ & KNDMC5C16F6019328 & Kia Sedona & 2015 \\
$\mathbf{0 1 1}$ & 4T1BK1EB5DU003862 & Toyota Avalon & 2013 \\
$\mathbf{0 1 2}$ & 1HGCT1B38DA000184 & Honda Accord & 2013 \\
$\mathbf{0 1 3}$ & JM3KE4BE0D0100445 & Mazda CX-5 & 2013 \\
$\mathbf{0 1 4}$ & 3N1CN7AP5CL809250 & Nissan Versa & 2012 \\
$\mathbf{0 1 5}$ & 3TMJU4GN0AM093246 & Toyota Tacoma & 2010 \\
$\mathbf{0 1 6}$ & WAUAFAFC6CN003676 & Audi A6 & 2012 \\
$\mathbf{0 1 7}$ & JTKJF5C70B3001720 & Scion tC & 2011 \\
$\mathbf{0 1 8}$ & ML32A3HJ6EH003209 & Mitsubishi Mirage & 2014 \\
$\mathbf{0 1 9}$ & WVWJK73C99P049019 & Volkswagen Passat & 2009 \\
$\mathbf{0 2 0}$ & 1HGCR2F37DA011773 & Honda Accord & 2013 \\
$\mathbf{0 2 1}$ & 2HGFG12607H511521 & Honda Civic & 2007 \\
$\mathbf{0 2 2}$ & 4JGCB65E59A094913 & Mercedes R-Class & 2009 \\
$\mathbf{0 2 3}$ & WBAVL1C56DVR91057 & BMW X1 & 2013 \\
$\mathbf{0 2 4}$ & ML32A3HJ4EH003547 & Mitsubishi Mirage & 2014 \\
$\mathbf{0 2 5}$ & KNAFW4A34A5148033 & Kia Forte & 2010 \\
$\mathbf{0 2 6}$ & WAUGNAF49HN009162 & Audi A4 & 2017 \\
$\mathbf{0 2 7}$ & KNADM4A34D6227438 & Kia Rio & 2013 \\
$\mathbf{0 2 8}$ & 2HGFB2F52CH300384 & Honda Civic & 2012 \\
$\mathbf{0 2 9}$ & 5TFEY5F18GX197447 & Toyota Tundra & 2016 \\
$\mathbf{0 3 0}$ & JM1BL1K53B1445800 & Mazda 3 & 2011 \\
$\mathbf{0 3 1}$ & 5NPDH4AEXBH019866 & Hyundai Elantra & 2011 \\
$\mathbf{0 3 2}$ & KNAFX4A65E5055079 & Kia Forte & 2014 \\
$\mathbf{0 3 3}$ & JF1ZNAA11D2703970 & Scion FR-S & 2013 \\
$\mathbf{0 3 4}$ & 2T1KU40E69C102910 & Toyota Matrix & 2009 \\
$\mathbf{0 3 5}$ & JTMBD33V065032593 & Toyota RAV4 & 2006 \\
$\mathbf{0 3 6}$ & 5TFRM5F14BX023073 & Toyota Tundra & 2011 \\
\hline & & & \\
\hline
\end{tabular}




\begin{tabular}{|c|c|c|c|}
\hline $\begin{array}{c}\text { Sample } \\
\text { ID }\end{array}$ & VIN & Vehicle Make \& Model & Year \\
\hline 037 & 5TDZA23C55S339489 & Toyota Sienna & 2005 \\
\hline 038 & KNADM4A37D6178283 & Kia Rio & 2013 \\
\hline 039 & JM1DKFB72G0129070 & Mazda CX-3 & 2016 \\
\hline 040 & WA1EFCFS2GR000662 & Audi Q3 & 2016 \\
\hline 041 & KNAFU4A29A5143195 & Kia Forte & 2010 \\
\hline 042 & WBA1F5C58EVV98871 & BMW 2 Series & 2014 \\
\hline 043 & 3MYDLBZV8GY100139 & Scion iA & 2016 \\
\hline 044 & 1N4AA6AP7GC380842 & Nissan Maxima & 2016 \\
\hline 045 & 4T1BK36B96U135066 & Toyota Avalon & 2006 \\
\hline 046 & WBA3A9C59CF270171 & BMW 3 Series & 2012 \\
\hline 047 & 5FNYF4H41CB001552 & Honda Pilot & 2012 \\
\hline 048 & 5N1AZ2MH3FN202392 & Nissan Murano & 2015 \\
\hline 049 & JHMGE88209S009320 & Honda Fit & 2009 \\
\hline 050 & KMHTC6AD8EU195530 & Hyundai Veloster & 2014 \\
\hline 051 & JM1CW2BL7E0166862 & Mazda 5 & 2014 \\
\hline 052 & 2HGFC4B51GH301966 & Honda Civic & 2016 \\
\hline 053 & 5TFEY5F14GX199602 & Toyota Tundra & 2016 \\
\hline 054 & JM1DKFC70G0136470 & Mazda CX-3 & 2016 \\
\hline 055 & JM1DKFC79G0136435 & Mazda CX-3 & 2016 \\
\hline 056 & 1G1ZB5EB5A4129282 & Chevrolet Malibu & 2010 \\
\hline 057 & 1VWAT7A37GC002279 & Volkswagen Passat & 2016 \\
\hline 058 & JTDKARFUXG3001654 & Toyota Prius & 2016 \\
\hline 059 & JA32U2FU0EU010058 & Mitsubishi Lancer & 2014 \\
\hline 060 & 1G1ZB5EB0AF210940 & Chevrolet Malibu & 2010 \\
\hline 061 & 3FA6P08D7HR103611 & Ford Fusion & 2017 \\
\hline 062 & 2LMTJ8KRXGBL39605 & Lincoln MKX & 2016 \\
\hline 063 & KMHD74LF5HU166048 & Hyundai Elantra & 2017 \\
\hline 064 & 4A3AB36FX9E043222 & Mitsubishi Galant & 2009 \\
\hline 065 & 4A3AB36F49E015450 & Mitsubishi Galant & 2009 \\
\hline 066 & 4A3AB36F39E029145 & Mitsubishi Galant & 2009 \\
\hline 067 & 2C3CCAAGXFH842181 & Chrysler 300 & 2015 \\
\hline 068 & 2HGFG3B59DH501522 & Honda Civic & 2013 \\
\hline 069 & 2HGFB2F50DH504344 & Honda Civic & 2013 \\
\hline 070 & JHMZF1D43BS008964 & Honda CR-Z & 2011 \\
\hline 071 & 5J6RM4H35CL072875 & Honda CR-Z & 2012 \\
\hline 072 & JHMZF1D48BS007552 & Honda CR-Z & 2011 \\
\hline 073 & 2HKRM4H38FH627963 & Honda CR-Z & 2015 \\
\hline 074 & 2HGFC2F52GH504878 & Honda Civic & 2016 \\
\hline 075 & 5J6RM4H34FL000599 & Honda CR-Z & 2015 \\
\hline
\end{tabular}




\begin{tabular}{|c|c|c|c|}
\hline $\begin{array}{c}\text { Sample } \\
\text { ID } \\
\end{array}$ & VIN & Vehicle Make \& Model & Year \\
\hline 076 & 3CZRU6H39GM717785 & Honda HR-V & 2016 \\
\hline 077 & 3HGGK5H58FM712771 & Honda Fit & 2015 \\
\hline 078 & 2HGFG21506H707035 & Honda Civic & 2006 \\
\hline 079 & 1FA6P8CF3G5236374 & Ford Mustang & 2016 \\
\hline 080 & 1FTEX1CF1GFA20367 & Ford F-150 & 2016 \\
\hline 081 & 1FTEX1CF7FFB30998 & Ford F-150 & 2015 \\
\hline 082 & 2FMTK4J85FBB64687 & Ford Edge & 2015 \\
\hline 083 & KL4CJFSB7FB044306 & Buick Encore & 2015 \\
\hline 084 & 1LNHL9DK9EG608524 & Lincoln MKS & 2014 \\
\hline 085 & 1G1RE6E47EU140143 & Chevrolet Volt & 2014 \\
\hline 086 & 1GCRCREC3GZ171577 & Chevrolet Silverado 1500 & 2016 \\
\hline 087 & 1G1ZC5E0XAF215588 & Chevrolet Malibu & 2010 \\
\hline 088 & YS3FD79Y876001896 & Saab 9-3 & 2007 \\
\hline 089 & 1G1JC5SH2F4177056 & Chevrolet Sonic & 2015 \\
\hline 090 & KNDMC5C14F6018761 & Kia Sedona & 2015 \\
\hline 091 & 5XYPHDA55GG004235 & Kia Sorento & 2016 \\
\hline 092 & KNDJN2A23F7115161 & Kia Soul & 2015 \\
\hline 093 & KNAFK4A61F5256185 & Kia Forte & 2015 \\
\hline 094 & KNDJT2A69C7365668 & Kia Soul & 2012 \\
\hline 095 & KNADN5A31C6064782 & Kia Rio & 2012 \\
\hline 096 & KNADM4A35D6250775 & Kia Rio & 2013 \\
\hline 097 & KNAFU4A23A5809774 & Kia Forte & 2010 \\
\hline 098 & KNAFU4A20A5061193 & Kia Forte & 2010 \\
\hline 099 & KNAFU4A24A5196726 & Kia Forte & 2010 \\
\hline 100 & KNDUP131646544318 & Kia Sedona & 2004 \\
\hline 101 & KNDJC735685790186 & Kia Sorento & 2008 \\
\hline 102 & KNDMB233466028628 & Kia Sedona & 2006 \\
\hline 103 & KNDJC733855460026 & Kia Sorento & 2005 \\
\hline 104 & 1C3CCCAB4FN500832 & Chrysler 200 & 2015 \\
\hline 105 & JA4AZ3A30EZ000940 & Mitsubishi Outlander & 2014 \\
\hline 106 & ML32A3HJ1EH003425 & Mitsubishi Mirage & 2014 \\
\hline 107 & 2B3KA43DX9H576961 & Dodge Charger & 2009 \\
\hline 108 & 1C4RDJAG4FC709700 & Dodge Durango & 2015 \\
\hline 109 & JA3AU26U88U042188 & Mitsubishi Lancer & 2008 \\
\hline 110 & 4A3AB36F39E024088 & Mitsubishi Galant & 2009 \\
\hline 111 & 4A3AB36F29E039116 & Mitsubishi Galant & 2009 \\
\hline 112 & JA4AR4AU9CZ000785 & Mitsubishi Outlander & 2012 \\
\hline 113 & JM1BM1L7XE1140067 & Mazda 3 & 2014 \\
\hline 114 & JM1DE1KY7D0166100 & Mazda 2 & 2014 \\
\hline
\end{tabular}




\begin{tabular}{|c|c|c|c|}
\hline $\begin{array}{c}\text { Sample } \\
\text { ID }\end{array}$ & VIN & Vehicle Make \& Model & Year \\
\hline 115 & JM1CW2BL1E0169143 & Mazda 5 & 2015 \\
\hline 116 & 1FADP5AU6EL509856 & Ford C-Max Hybrid & 2014 \\
\hline 117 & JM1DE1HYXB0105280 & Mazda 2 & 2011 \\
\hline 118 & JM1BK323761529766 & Mazda 3 & 2006 \\
\hline 119 & JM1GJ1V51E1101202 & Mazda 6 & 2014 \\
\hline 120 & 1YVHZ8DH8C5M40525 & Mazda 6 & 2012 \\
\hline 121 & 1N4AL2AP0AN450308 & Nissan Altima & 2010 \\
\hline 122 & KMHDU4AD8AU171938 & Hyundai Elantra & 2010 \\
\hline 123 & JS2YC415585103206 & Suzuki SX4 & 2008 \\
\hline 124 & JS2RE9A32A6101695 & Suzuki Kizashi & 2010 \\
\hline 125 & JS2RE9A36C6101041 & Suzuki Kizashi & 2012 \\
\hline 126 & 1N4AL3AP5DC153542 & Nissan Altima & 2013 \\
\hline 127 & 1N4AL11D46N373837 & Nissan Altima & 2006 \\
\hline 128 & 1N4AL3AP0GC123773 & Nissan Altima & 2016 \\
\hline 129 & 1N4AA6AP3GC378439 & Nissan Maxima & 2016 \\
\hline 130 & 3N1BC13E57L352925 & Nissan Versa & 2007 \\
\hline 131 & JN8AF5MR1BT006565 & Nissan Juke & 2011 \\
\hline 132 & JN8AE2KP1E9107979 & Nissan Quest & 2014 \\
\hline 133 & 5N1AZ2MH8FN203215 & Nissan Murano & 2015 \\
\hline 134 & 5N1AR2MM0FC604332 & Nissan Pathfinder & 2015 \\
\hline 135 & 3N1AB61E78L637820 & Nissan Sentra & 2008 \\
\hline 136 & 3N1CN7AP8EL809326 & Nissan Versa & 2014 \\
\hline 137 & JN8AF5MR6ET354981 & Nissan Juke & 2014 \\
\hline 138 & 3N1AB7AP6FY215175 & Nissan Sentra & 2015 \\
\hline 139 & 1N4AZ0CP2ЕС330348 & Nissan Leaf & 2014 \\
\hline 140 & 3N1AB61EX8L637586 & Nissan Sentra & 2008 \\
\hline 141 & JN8AZ28R59T100782 & Nissan Cube & 2009 \\
\hline 142 & 1N4AL21E49C197032 & Nissan Altima & 2009 \\
\hline 143 & WAUAFAFL0CA118555 & Audi A4 & 2012 \\
\hline 144 & WVWLK73C87E003165 & Volkswagen Passat & 2007 \\
\hline 145 & KM8SMDHF5FU099649 & Hyundai Santa Fe & 2015 \\
\hline 146 & KM8JUCAC9AU071268 & Hyundai Tucson & 2010 \\
\hline 147 & 5NPEU46F36H004326 & Hyundai Sonata & 2006 \\
\hline 148 & 5NPE24AA4GH265905 & Hyundai Sonata & 2016 \\
\hline 149 & KMHTC6AD9EU195701 & Hyundai Veloster & 2014 \\
\hline 150 & KM8J3CA44GU039705 & Hyundai Tucson & 2016 \\
\hline 151 & KMHCT4AE5DU423849 & Hyundai Accent & 2013 \\
\hline 152 & KMHGN4JE1FU012205 & Hyundai Genesis & 2015 \\
\hline 153 & KMHGC46E59U043461 & Hyundai Genesis & 2009 \\
\hline
\end{tabular}




\begin{tabular}{|c|c|c|c|}
\hline $\begin{array}{c}\text { Sample } \\
\text { ID }\end{array}$ & VIN & Vehicle Make \& Model & Year \\
\hline 154 & 5NPE24AA7GH327619 & Hyundai Sonata & 2016 \\
\hline 155 & KM8JN72D26U380194 & Hyundai Tucson & 2006 \\
\hline 156 & 5NPE24AF0FH005401 & Hyundai Sonata & 2015 \\
\hline 157 & WAUFFAFC6GN005150 & Audi A6 & 2016 \\
\hline 158 & 1VWAP7A32CC062775 & Volkswagen Passat & 2012 \\
\hline 159 & WVGBV3AX7DW592597 & Volkswagen Tiguan & 2013 \\
\hline 160 & 3VW2K7AJ4CM385273 & Volkswagen Jetta & 2012 \\
\hline 161 & WVGBV75N99W000785 & Volkswagen Tiguan & 2009 \\
\hline 162 & 3VWJP7AT2DM675477 & Volkswagen Beetle & 2013 \\
\hline 163 & JN1DV6AP2CM811431 & Infiniti $G$ & 2012 \\
\hline 164 & 3FAHP06Z17R191545 & Ford Fusion & 2007 \\
\hline 165 & JH4KB2F56AC000729 & Acura RL & 2010 \\
\hline 166 & 19UUB2F33FA005996 & Acura TLX & 2015 \\
\hline 167 & 19UUB2F75FA001855 & Acura TLX & 2015 \\
\hline 168 & 5J8TB4H34GL002597 & Acura RDX & 2016 \\
\hline 169 & 2LMTJ8LR7GBL47532 & Lincoln MKX & 2016 \\
\hline 170 & 19UUA8F23CA021785 & Acura TL & 2012 \\
\hline 171 & 19UUA8F20CA012719 & Acura TL & 2012 \\
\hline 172 & JH4CU2F44CC004593 & Acura TSX & 2012 \\
\hline 173 & JH4KC1F38EC002509 & Acura RLX & 2014 \\
\hline 174 & YV1MK672X92146049 & Volvo C30 & 2009 \\
\hline 175 & YV4982DZ2A2058523 & Volvo XC60 & 2010 \\
\hline 176 & YV1MC68267J021595 & Volvo C70 & 2007 \\
\hline 177 & YV1622FS3C2037006 & Volvo S60 & 2012 \\
\hline 178 & YV4CZ592361284695 & Volvo XC90 & 2006 \\
\hline 179 & YV1622FS1C2087628 & Volvo S60 & 2012 \\
\hline 180 & YV1622FS0C2102782 & Volvo S60 & 2012 \\
\hline 181 & YV4952CZ9E1684771 & Volvo XC90 & 2014 \\
\hline 182 & YV1952AS0E1179656 & Volvo S80 & 2014 \\
\hline 183 & WBA3A5C55CF259029 & BMW 3 Series & 2012 \\
\hline 184 & WMWXM5C55ET936691 & Mini Cooper & 2014 \\
\hline 185 & WBA1F5C59EVV98894 & BMW 2 Series & 2014 \\
\hline 186 & WBA5B1C52ED484411 & BMW 5 Series & 2014 \\
\hline 187 & WBAVL1C58DVR88063 & BMW X1 & 2013 \\
\hline 188 & WMWZC3C52BWH97939 & Mini Cooper Countryman & 2011 \\
\hline 189 & WDDGF4HB6DR283277 & Mercedes C Class & 2013 \\
\hline 190 & WDDGF4HB4CR213372 & Mercedes C Class & 2012 \\
\hline 191 & 5TFRY5F12FX187772 & Toyota Tundra & 2015 \\
\hline 192 & JTEBU5JR5E5155284 & Toyota 4Runner & 2014 \\
\hline
\end{tabular}




\begin{tabular}{cccc}
\hline $\begin{array}{c}\text { Sample } \\
\text { ID }\end{array}$ & VIN & Vehicle Make \& Model & Year \\
\hline $\mathbf{1 9 3}$ & JF1GD75647G505362 & Subaru Impreza & 2007 \\
$\mathbf{1 9 4}$ & JF2SH6AC4AH737008 & Subaru Forester & 2010 \\
$\mathbf{1 9 5}$ & JF1GD67656H504575 & Subaru Impreza & 2006 \\
$\mathbf{1 9 6}$ & 4S3BNAA61F3003502 & Subaru Legacy & 2015 \\
$\mathbf{1 9 7}$ & JF1GE61659H515022 & Subaru Impreza & 2009 \\
$\mathbf{1 9 8}$ & 4S4WX82C864430559 & Subaru B9 Tribeca & 2009 \\
$\mathbf{1 9 9}$ & JF2SJADC7EH400163 & Subaru Forester & 2014 \\
$\mathbf{2 0 0}$ & 4S3BNAA69F3003523 & Subaru Legacy & 2015 \\
$\mathbf{2 0 1}$ & JF1GE61658H503418 & Subaru Impreza & 2008 \\
$\mathbf{2 0 2}$ & JF2SJAAC0EH409856 & Subaru Forester & 2014 \\
$\mathbf{2 0 3}$ & JF2SH61699H704494 & Subaru Forester & 2009 \\
$\mathbf{2 0 4}$ & 4S3BMCA65C3032608 & Subaru Legacy & 2012 \\
$\mathbf{2 0 5}$ & JF1GH61699H816786 & Subaru Impreza & 2009 \\
$\mathbf{2 0 6}$ & JF1GPAA63EH203000 & Subaru Impreza & 2014 \\
$\mathbf{2 0 7}$ & 3C4PDDBG3FT598049 & Dodge Journey & 2015 \\
$\mathbf{2 0 8}$ & ZACCJBAT1FPC09734 & Jeep Renegade & 2015 \\
$\mathbf{2 0 9}$ & ZACCJBAT5FPC27590 & Jeep Renegade & 2015 \\
$\mathbf{2 1 0}$ & 2C3CDZBT9GH108611 & Dodge Challenger & 2016 \\
\hline
\end{tabular}


VITA

\section{RUTHMARA CORZO}

Born Miami, Florida, USA

2013

Certificate in Forensic Science

Florida International University

Miami, Florida

2013

BS in Chemistry

Florida International University

Miami, Florida

2013-2016

Presidential Fellowship

Florida International University

Miami, Florida

2016

MS in Chemistry

Florida International University

Miami, Florida

2016-2018

Doctoral Candidate

Florida International University

Miami, Florida

\section{PUBLICATIONS AND PRESENTATIONS}

R. CORZO, J. R. Almirall. The discrimination of printing inks by Scanning Electron Microscopy-Energy Dispersive X-Ray Spectroscopy. International Forensic Research Institute Symposium $3^{\text {rd }}$ Annual Forensic Science Symposium, May 2014, Miami, Florida, USA.

R. CORZO, J. R. Almirall. The Discrimination of Printing Inks using Laser Induced Breakdown Spectroscopy for Forensic Applications. SciX Conference, Providence, September 2015, Rhode Island, USA.

R. CORZO, T. Hoffman, J. R. Almirall. Development of a LIBS Database for the Forensic Interpretation of Glass Evidence. First Industry Advisory Board (IAB) Meeting, August 2017, Miami, Florida, USA. 
R. CORZO, T. Hoffman, P. Weis, J. Franco-Pedroso, D. Ramos, J. Almirall. The Use of LA-ICP-MS Databases to Estimate Likelihood Ratios for the Forensic Analysis of Glass Evidence. Talanta (2018), https://doi.org/10.1016/j.talanta.2018.02.027

R. CORZO, S. C. Jantzi, C. L. Dutton, A. K. Saha, J. R. Almirall. Filter Pellet Sample Preparation for Analysis of Filter-Bound Sediments by LA-ICP-MS for Fingerprinting, September 2016, SciX Conference, Minneapolis, Minnesota, USA.

R. CORZO, D. Ramos, J. Franco-Pedroso, P. Weis, J. R. Almirall. Calculation of Likelihood Ratios for Glass Evidence. 23 ${ }^{\text {rd }}$ Annual EPG Meeting, September 2017, Athens, Greece.

R. CORZO, D. Ramos, J. Franco-Pedroso, P. Weis, J. R. Almirall. The Use of LA-ICPMS Databases to Estimate Likelihood Ratios for the Forensic Evaluation of Glass Evidence. SciX Conference, October 2017, Reno, Nevada, USA.

R. CORZO, K. Subedi, T. Trejos, J. R. Almirall. The Discrimination of Printing Inks by Spectroscopic and Mass Spectrometric Techniques, September 2014, SciX Conference, Reno, Nevada, USA.

R. CORZO, K. Subedi, T. Trejos, J. Almirall. Evaluation of the Forensic Utility of Scanning Electron Microscopy-Energy Dispersive Spectroscopy and Laser AblationInductively Coupled Plasma-Mass Spectrometry for Printing Ink Examinations. $J$ Forensic Sci. 2016.

T. Hoffman, R. CORZO, J. Almirall. Strengthening the Significance of Glass Evidence through Inter-laboratory Tests: Design and Implementation of FIU Working Groups. NIST 2nd Colloquium on the Weight of Evidence, June 2017, Gaithersburg, MD, USA.

C. Johnson, P. Martin, K. A. Roberts, T. Trejos, R. CORZO, J. Almirall, A. Safer. The Capability of Raman Microspectroscopy to Differentiate Printing Inks. J of Forensic Sci. 2017.

T. Trejos, R. CORZO, K. Subedi, J. Almirall. Characterization of toners and inkjets by laser ablation spectrochemical methods and Scanning Electron Microscopy-Energy Dispersive X-ray Spectroscopy. Spectrochim Acta B. 2014.

T. Trejos, P. Torrione, R. CORZO, A. Raeva, K. Subedi, R. Williamson, J. H. Yoo, J. Almirall. A novel forensic tool for the characterization and comparison of printing ink evidence: development and evaluation of a searchable database using data fusion of spectrochemical methods. J Forensic Sci. 2016. 